NISTIR 8395

\title{
Low-GWP Alternative Refrigerant Blends for HFC-134a: Interim Report
}

\author{
Strategic Environmental Research and Development Program \\ Project: WP 19-1385
}

Principal Investigators:

Piotr A. Domanski

Mark O. McLinden

Co-Authors:

Valeri I. Babushok

Ian H. Bell

Tara J. Fortin

Michael J. Hegetschweiler

Mark A. Kedzierski

Dennis K. Kim

Lingnan Lin

Gregory T. Linteris

Stephanie L. Outcalt

Richard A. Perkins

Aaron Rowane

Harrison Skye 



\title{
Low-GWP Alternative Refrigerant Blends for HFC-134a: Interim Report
}

\author{
Interim Report \\ Strategic Environmental Research and Development Program \\ Project: WP 19-1385
}

\begin{abstract}
Piotr A. Domanski, Valeri I. Babushok, Michael J. Hegetschweiler, Lingnan Lin, Mark A. Kedzierski, Gregory T. Linteris, Harrison Skye

Building Energy and Environment Division Engineering Laboratory

Mark O. McLinden, Ian H. Bell, Tara J. Fortin, Stephanie Outcalt, Richard A. Perkins, Aaron Rowane

Applied Chemicals and Materials Division Material Measurement Laboratory
\end{abstract}

This publication is available free of charge from: https://doi.org/10.6028/NIST.IR.8395

October 2021

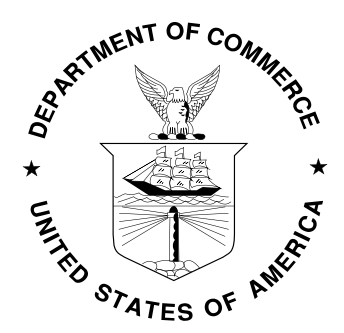

U.S. Department of Commerce Gina M. Raimondo, Secretary

National Institute of Standards and Technology James K. Olthoff, Performing the Non-Exclusive Functions and Duties of the Under Secretary of Commerce for Standards and Technology \& Director, National Institute of Standards and Technology 
Certain commercial entities, equipment, or materials may be identified in this document in order to describe an experimental procedure or concept adequately. Such identification is not intended to imply recommendation or endorsement by the National Institute of Standards and Technology, nor is it intended to imply that the entities, materials, or equipment are necessarily the best available for the purpose.

National Institute of Standards and Technology Interagency or Internal Report 8395 Natl. Inst. Stand. Technol. Interag. Intern. Rep. 8395, 177 pages (October 2021)

This publication is available free of charge from: https://doi.org/10.6028/NIST.IR.8395 


\begin{abstract}
This project addresses the objectives of the Statement of Need number WPSON-17-20 "No/Low Global Warming Potential Alternatives to Ozone Depleting Refrigerants." Its goal is to identify and demonstrate performance of low global-warming-potential (GWP), nonflammable refrigerants to replace HFC-134a in military environmental control units (ECUs). This project is a follow-on to the limited-scope project WP-2740, which used thermodynamic cycle simulation models alone to screen over 100000 refrigerant blends and identified over 20 candidate HFC-134a replacements. In this study we narrow the pool of blend candidates down to three 'best' fluids, verify experimentally their flammability behavior, demonstrate their performance through tests in a military ECU in environmental chambers over a wide range of operating conditions, and extrapolate the laboratory-measured performance to ECUs equipped with optimized heat exchangers through first-principles-based simulations combined with machine-learning optimization methods.

This Interim Report documents the work leading to the selection of three 'best' blends. This work included refrigerant blend tests in a laboratory mini-breadboard heat pump apparatus, fundamental measurements and modeling of thermophysical properties, two-phase heattransfer performance, and flammability behavior.
\end{abstract}

\title{
Key Words
}

Air conditioning; Coefficient of performance; Cycle simulation; Flammability; Refrigerants; Vapor compression; Volumetric capacity. 


\section{Table of Contents}

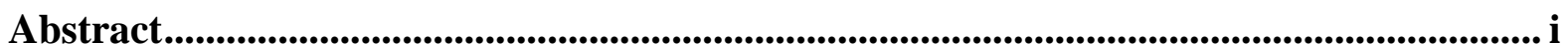

Key Words .........................................................................................................................................

List of Tables .......................................................................................................................

Nomenclature ........................................................................................................................ viii

Acknowledgements ................................................................................................................................ ix

1. Objective ............................................................................................................................... 1

2. Technical Approach ............................................................................................................ 2

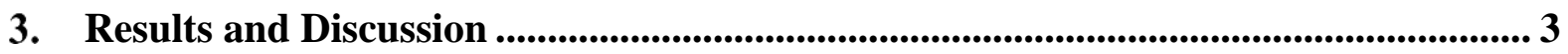

3.1. Task 1: Experimental Measurements of Blend Properties and Development of Mixture Equation of State ...................................................................................................... 3

3.1.1. Purpose and Approach................................................................................. 3

3.1.2. Vapor-Liquid Equilibrium (VLE) Measurements............................................. 5

3.1.3. Pressure-Density-Temperature $(P, \rho, T, x)$ Measurements................................... 5

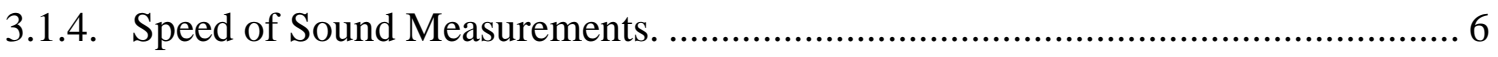

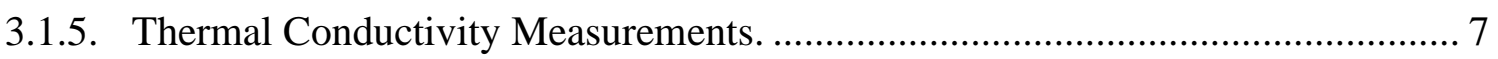

3.1.6. Viscosity Measurements............................................................................... 7

3.1.7. Model Development (Subtask 1c)................................................................. 7

3.2. Task 2: Flammability Testing ……................................................................................ 11

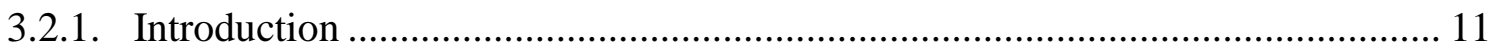

3.2.2. E681 tests and Japanese High-Pressure Gas Law tests ..................................... 12

3.2.3. Improvements in Predictive Model ................................................................ 20

3.2.4. Interpretation of E681 and JHPGL Experimental Results ................................. 22

3.2.5. Coordination with Full-Scale Testing ........................................................... 25

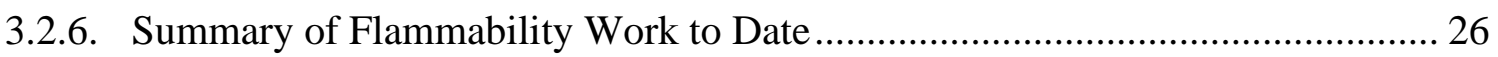

3.3. Task 3: Testing of Selected Blends in a Mini-Breadboard Heat Pump.............. 27

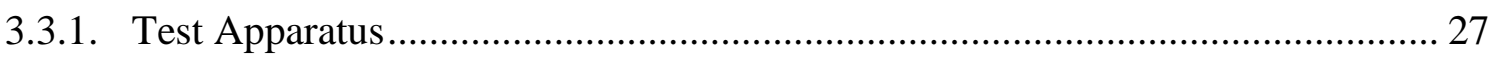

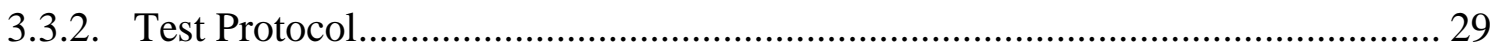

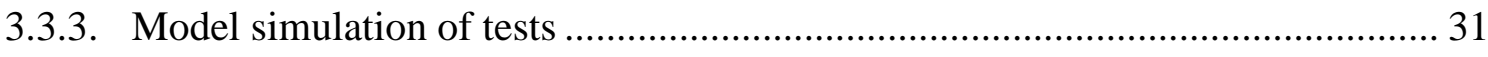

3.3.4. Test Results and CYCLE_D-HX Model Validation .......................................... 32

3.4. Task 4: Refrigerant Forced-Convection Heat-Transfer Testing .......................... 39

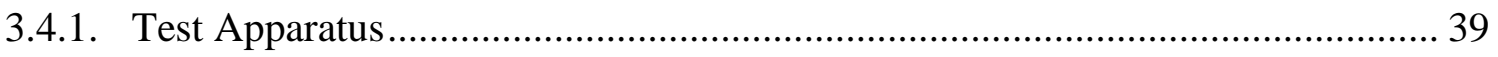

3.4.2. Data Analysis and Correlation Development .................................................. 42

3.5. Task 5: Selection of Final Blends for Testing in Military ECU ............................ 46 


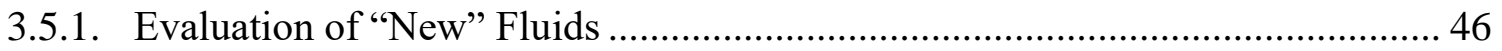

3.5.2. Selection of Four Blends for Testing in Mini-Breadboard Heat Pump............... 48

3.5.3. Selection of Three Blends for Testing in ECU................................................... 49

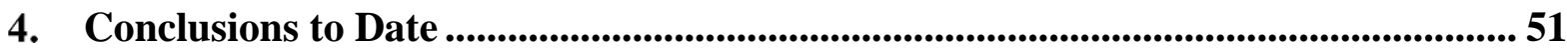

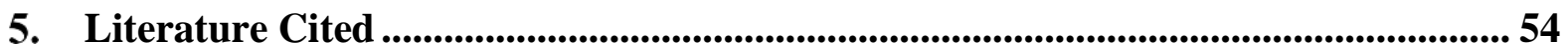

Appendix A: Details of Experiments and Modeling .........................................................60

A.1. Task 1 - Details of Thermophysical Properties Measurements and Modeling.... 60

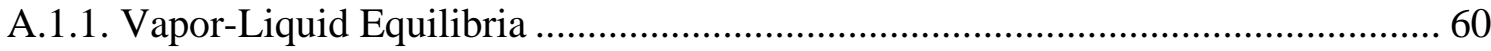

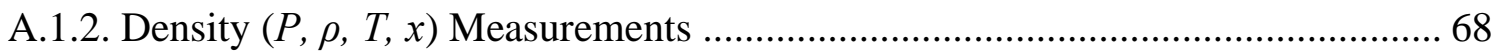

A.1.3. Liquid-Phase Speed of Sound.......................................................................... 91

A.1.4. Liquid-phase Thermal Conductivity ............................................................. 115

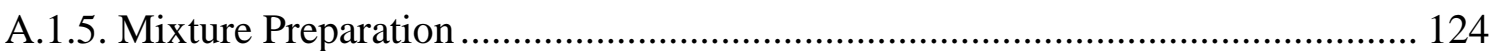

A.2. Task 2 - Flammability Experiments and Test Data ................................................ 128

A.2.1. ASHRAE Standard 34 E681 Test Data ........................................................ 128

A.2.2. Japanese High-Pressure Gas Law Test .......................................................... 131

A.2.3. Constant Volume (2 L) Laminar Burning Velocity Measurements ................... 139

A.3 Task 4 - Tabulated Flow-Boiling Heat-Transfer Measurements............................ 149

\section{List of Tables}

Table 3.1-1. Refrigerant blend properties measured in the current work. 4

Table 3.1-2. Interaction parameters used in this work (note: the order of components is

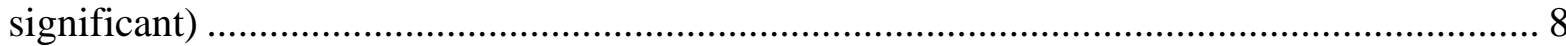

Table 3.1-3. Coefficients for entropy scaling of thermal conductivity data (Eq. 3.1-5)......... 10 Table 3.2-1. Modeling and test results for candidate blends (reactants at $296 \mathrm{~K}, 101 \mathrm{kPa}$, and $50 \%$ r.h.) 14

Table 3.3-1. MBHP: HFC-134a baseline test operating targets and control parameters........ 30

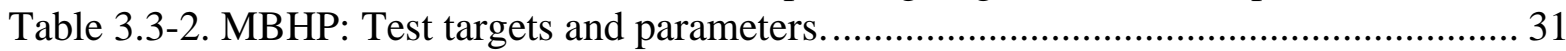

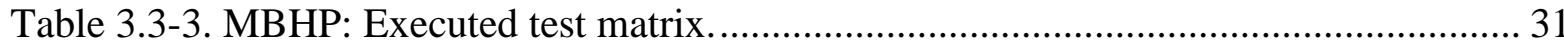

Table 3.5-13.5-2. Selected "best" blends from the limited-scope project (sorted by GWP) ${ }^{3} 49$ Table A.1-1. Measured vapor-liquid equilibria (VLE) data for the system R-1234yf/134a at a molar composition of $(0.320 / 0.680)$.

Table A.1-2. Measured vapor-liquid equilibria (VLE) data for the system R-1234yf/134a at a molar composition of $(0.647 / 0.353)$.

Table A.1-3. Measured vapor-liquid equilibria (VLE) data for the system R-134a/1234ze(E) at a molar composition of $(0.334 / 0.666)$.

Table A.1-4. Measured vapor-liquid equilibria (VLE) data for the system R-134a/1234ze(E) at a molar composition of $(0.663 / 0.337)$ 64

Table A.1-5. Measured vapor-liquid equilibria (VLE) data for the system R$1234 \mathrm{yf} / 1234 \mathrm{ze}(\mathrm{E})$ at a molar composition of $(0.324 / 0.676)$. 
Table A.1-6. Measured vapor-liquid equilibria (VLE) data for the system R$1234 \mathrm{yf} / 1234 z e(\mathrm{E})$ at a molar composition of $(0.638 / 0.362)$.

Table A.1-7. Measured vapor-liquid equilibria (VLE) data for the system R125/1234yf at a molar composition of $(0.349 / 0.651)$.

Table A.1-8. Measured vapor-liquid equilibria (VLE) data for the system R-125/1234yf at a

molar composition of $(0.664 / 0.336)$.

Table A.1-9. Measured $(P, \rho, T, x)$ data for the system R-1234yf/134a at a molar composition of $(0.33634 / 0.66366)$. 72

Table A.1-10. Measured $(P, \rho, T, x)$ data for the system R-1234yf/134a at a molar composition of $(0.64709 / 0.35291)$. 76

Table A.1-11. Measured $(P, \rho, T, x)$ data for the system R-134a/1234ze(E) at a molar composition of $(0.33250 / 0.66750)$.

Table A.1-12. Measured $(P, \rho, T, x)$ data for the system R-134a/1234ze(E) at a molar composition of $(0.66356 / 0.33644)$.

Table A.1-13. Measured $(P, \rho, T, x)$ data for the system R-1234yf/1234ze(E) at a molar composition of $(0.33584 / 0.66416)$.

Table A.1-14. Measured $(P, \rho, T, x)$ data for the system R-1234yf/1234ze(E) at a molar composition of $(0.66660 / 0.33340)$.

Table A.1-15. Measured speed of sound data for the system R-1234yf/134a at a molar composition of $(0.33634 / 0.66366)$ 96

Table A.1-16. Measured speed of sound data for the system R-1234yf/134a at a molar composition of $(0.64709 / 0.35291)$.

Table A.1-17. Measured speed of sound data for the system R-134a/1234ze(E) at a molar composition of $(0.32916 / 0.67084)$.

Table A.1-18. Measured speed of sound data for the system R-134a/1234ze(E) at a molar composition of $(0.63688 / 36312)$.

Table A.1-19. Measured speed of sound data for the system R-1234yf/1234ze(E) at a molar composition of $(0.33584 / 0.66416)$ 110

Table A.1-20. Measured speed of sound data for the system R-1234yf/1234ze(E) at a molar composition of $(0.66660 / 0.33340)$. 113

Table A.1-21. Representative thermal conductivity data measured for the system R$1234 \mathrm{yf} / 134 \mathrm{a}$ at a molar composition of (0.320/0.680).

Table A.1-22. Representative thermal conductivity data measured for the system R$1234 \mathrm{yf} / 134 \mathrm{a}$ at a molar composition of $(0.647 / 0.353)$.

Table A.1-23. Representative thermal conductivity data measured for the system R$134 \mathrm{a} / 1234 \mathrm{ze}(\mathrm{E}))$ at a molar composition of $(0.334 / 0.666)$.

Table A.1-24. Representative thermal conductivity data measured for the system R$134 \mathrm{a} / 1234 \mathrm{ze}(\mathrm{E}))$ at a molar composition of $(0.663 / 0.337)$.

Table A.1-25. Representative thermal conductivity data measured for the system R$1234 \mathrm{yf} / 1234 \mathrm{ze}(\mathrm{E})$ at a molar composition of $(0.323 / 0.677)$.

Table A.1-26. Representative thermal conductivity data measured for the system R$1234 \mathrm{yf} / 1234 \mathrm{ze}(\mathrm{E})$ at a molar composition of (0.642/0.358).... 123

Table A.2-1. E681 test results for R-513A, NIST-ternary blend, R-450A, R-515B, and blends of R-1234yf/134a at a molar composition of $(0.26 / 0.74),(0.28 / 0.72)$, and $(0.30 / 0.70) \ldots \ldots .128$ 
Table A.2-2. Japanese High-Pressure Gas Law test results obtained at NIST for: R-513A, NIST-ternary blend, R-450A, R-515B, and blends of R-1234yf/134a, all with dry or $50 \%$ r.h. air as listed.

Table A.2-3. Fitting parameters for the variation of $S L$ with $\phi, T_{\mathrm{u}}$ and $P$ for experimental burning velocity data obtained in 2-L experiment from pressure rise data for R-152a/134a and R-152a/1234yf mixtures. Data presented for $298 \mathrm{~K}, 101 \mathrm{kPa}$ initial conditions, and dry air $(<2 \%$ r.h.) of wet air ( $50 \%$ r.h.) as noted.

Table A.2-4. Experimental burning velocity data obtained in 2-L experiment from pressure rise data for R-152a/134a and R-152a/1234yf mixtures. Data presented for $298 \mathrm{~K}, 101 \mathrm{kPa}$ initial conditions, dry air (<2\% r.h.) and wet air (50\% r.h.) as noted. Blend compositions given in mole fraction.

Table A.3-1. Measured flow-boiling heat-transfer data for R-515B, R-450A, R-513A, and HFC-134a.

\section{List of Figures}

Figure 1.0-1. Project tasks 1

Figure 3.1-1. Measured $(P, \rho, T, x)$ points for the R-1234yf/1234ze(E) blend at a composition of $(0.6666 / 0.3334)$ molar; left: pressure versus temperature; right: density versus temperature; $\diamond$, measured points; line, phase boundary; *, critical point. 5

Figure 3.1-2. Measured speed of sound points for the R-1234yf/1234ze(E) blend at a composition of $(0.6666 / 0.3334)$ mole fraction; left: pressure versus temperature; right: sound speed versus temperature; symbols are measured points and lines connect points along an isochore; red line is phase boundary.

Figure 3.1-3. Deviation plots for the measured experimental data with the mixture EOS using the parameters from Table 3.1-2. The different plot symbols indicate the two compositions measured here; the vertical lines indicate the mixture critical temperature. Literature data are shown as light grey + markers (some literature data have deviations outside the range of the figures).

Figure 3.1-4. Entropy scaling model for the thermal conductivity of the three blends studied here; (a) plus-scaled thermal conductivity $\lambda+$ as a function of reduced scaled residual entropy $s+$, the different symbols denote the different measured blends; (b) relative deviations in $\lambda+$.

Figure 3.2-1. Left image: schematic of ASTM 681 test vessel; Right two images: visual images from HFC-32/HFC-134a blend (0.35/0.65 mass fraction) below and above the lean flammability limit 20,21 ......

Figure 3.2-2. Equilibrium explosion pressure of candidate blends (Tern-1, R-513A, R-450A, and R-515B) together with experimental results in the JHPGL test as a function of blend volume fraction in air (50\% r.h.).

Figure 3.2-3. Explosion pressure of candidate blends in JHPGL test as a function of blend volume fraction in air (left frame: dry air; right, moist).

Figure 3.2-4. Explosion pressure of candidate blends (Tern-1, R-513A, R-450A, and $\mathrm{R}-515 \mathrm{~B}$ ) in JHPGL test as a function of blend volume fraction in air (solid lines: dry air; dashed lines: moist air; note different scales). 
Figure 3.2-5. Explosion pressure in the JHPGL test for binary blends of HFC-134a with HFO-1234yf as function of volume fraction the blend in air. Different curves show results for varying volume fractions of HFC-134a/HFO-1234yf in the blend (left frame: dry air; right: moist).

Figure 3.2-6. Peak explosion pressure in the JHPGL test for binary blends of HFC-134a with HFO-1234yf as function the volume fraction of HFC-134a in the blend, for moist (50 \% r.h.) and dry $(<2 \%$ r.h.) air.

Figure 3.2-7. Laminar burning velocity of R-152a/134a (left frame) and R-152a/1234yf (right frame) mixtures with air as a function of the equivalence ratio.

Figure 3.2-8. Calculated vs. measured laminar burning velocity (peak over all $\phi$ ) of twentyone refrigerant $/ \mathrm{N}_{2} / \mathrm{O}_{2}$ flames for which experimental data are available in the literature ${ }^{46-48}$.

Figure 3.2-9. Overall chemical reaction $\omega_{\mathrm{psr}}$ as a function of volume fraction in air determined via perfectly stirred reactor simulations for the blend R-1234yf/134a. Each curve refers to one blend ratio: top curve, 100\% HFO-1234yf, bottom curve $100 \%$ HFC-134a;

Frames: $0 \%$ r.h. (dry), $50 \%$ r.h., and $100 \%$ r.h. 22 Figure 3.2-10. Peak (over all $\phi$ for each blend) chemical reaction $\omega_{\mathrm{psr}}$ as a function of volume fraction of HFC-134a in the blend of R-1234yf/134a. Individual curves are shown for varying water vapor in the air $(0 \%, 50 \%, 75 \%$ and $100 \%$ r.h. $)$....

Figure 3.2-11. Explosion pressure in the JHPGL test vs. overall chemical reaction $\omega_{\text {psr }}$ (both peak over all $\phi$ ) for R-513A, R-450A, R-515B and the Tern-1 blend and the R-1234yf/134a blends at increasing HFC-134a volume fraction. Dotted line is a polynomial fit to all the data to aid in visualization. (Initial conditions: $296 \mathrm{~K}, 101 \mathrm{kPa}, 50 \%$ r.h.) 24 Figure 3.2-12. Peak (over all $\phi$ for each blend) chemical reaction $\omega_{\mathrm{psr}}$ for R-513A, R-450A, R-515B and the NIST blend. Data are shown for the Nominal, WCF, and WCFF compositions in $\operatorname{air}(T=296 \mathrm{~K})$ and $0 \%, 50 \%$, and $100 \%$ r.h.................................... 25

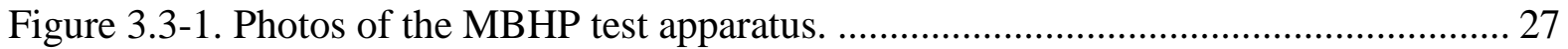

Figure 3.3-2. Schematic of the MBHP test apparatus.................................................... 28 Figure 3.3-3. MBHP: Schematics of annular heat exchanger including (a) refrigerant tube lengths, (b) cross section of annular heat exchanger, (c) detailed cross-section of microfin

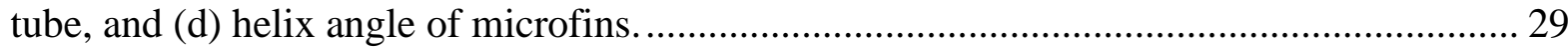
Figure 3.3-4. Cooling COP for HFC-134a and the replacement candidates (a) without LLSLHX and (b) with LLSL-HX.................................................................................. 34 Figure 3.3-5. Cooling volumetric capacity for HFC-134a and the replacement candidates (a) without LLSL-HX and (b) with LLSL-HX. ............................................................... 34 Figure 3.3-6. Compressor isentropic efficiency (a) without LLSL-HX and (b) with LLSL-

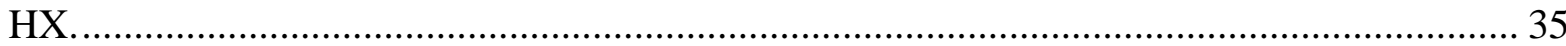

Figure 3.3-7. Compressor volumetric efficiency (a) without LLSL-HX and (b) with LLSL-

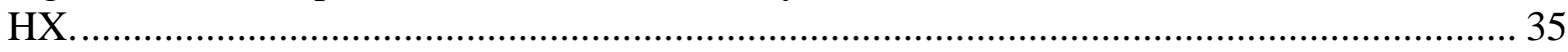

Figure 3.3-8. Compressor speed (a) without LLSL-HX and (b) with LLSL-HX................. 36

Figure 3.3-9. R-450A measurement and model prediction of: (a) evaporator and ................ 38

Figure $3.4-1$. Schematic of flow boiling test apparatus. ............................................... 40

Figure 3.4-2. Cross section of flow-boiling test section............................................... 41

Figure 3.4-3. Detailed schematic of test section (counterflow) ....................................... 41

Figure 3.4-4. Comparison between measured Nusselt numbers and those predicted by the Hamilton et al. correlation ${ }^{59}$...... 
Figure 3.4-5. Comparison between measured Nusselt numbers and those predicted by the

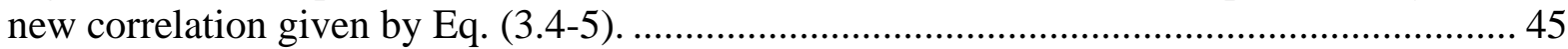

Figure A.1-1. Schematic of instrument for vapor liquid equilibria measurements. ............ 60

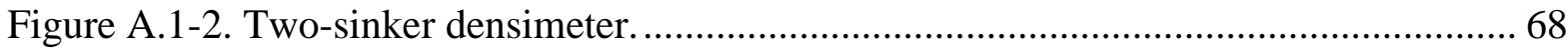

Figure A.1-3. Sinkers in the two-sinker densimeter (shown removed from the measuring

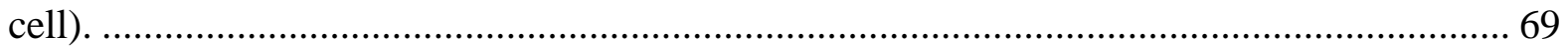

Figure A.1-4. Dual-path, pulse-echo, speed of sound instrument. .................................... 92

Figure A.1-5. Instrument rack for the pulse-echo speed of sound instrument..................... 93

Figure A.1-6. Schematic diagram of the measuring cell inside the pressure vessel............. 94

Figure A.1-7. Circuit diagram of the Wheatstone bridge and long and short hot wire arrangement.

Figure A.1-8. Effect of density on the thermal conductivity for binary mixtures of R-1234yf,

R-134a, and R-1234ze(E) at temperatures ranging from $200 \mathrm{~K}$ to $340 \mathrm{~K}$.

Figure A.2-1. Spherical 2-L chamber for JHPGL tests and constant volume method laminar

burning velocity measurements.

Figure A.2-2. Plumbing schematic diagram for 2-L chamber.

Figure A.2-3. Experimental pressure trace P, flame radius rf, flame stretch rate $\kappa$, and gas temperature (unburned $\mathrm{Tu}$ and burned $\mathrm{Tb}$ ) as a function of time.

Figure A.2-4. Three-dimensional plot of burning velocity as a function of pressure $\mathrm{P}$ and unburned gas temperature Tu.

\section{List of Acronyms}

$\begin{array}{ll}\text { AC } & \text { air conditioning } \\ \text { AHRI } & \text { Air-Conditioning, Heating and Refrigeration Institute } \\ \text { ANSI } & \text { American National Standards Institute } \\ \text { ARL } & \text { Army Research Laboratory } \\ \text { ASHRAE } & \text { American Society of Heating Refrigerating and Air-Conditioning Engineers } \\ \text { ASTM } & \text { American Society for Testing and Materials } \\ \text { CFC } & \text { chlorofluorocarbon } \\ \text { COP } & \text { coefficient of performance } \\ \text { ECU } & \text { environmental control unit } \\ \text { GWP } & \text { global warming potential } \\ \text { HFC } & \text { hydrofluorocarbon } \\ \text { HFO } & \text { hydroflouroolefin } \\ \text { HTF } & \text { heat-transfer fluid } \\ \text { HVAC } & \text { heating, ventilating and air-conditioning } \\ \text { ISO } & \text { International Organization for Standardization } \\ \text { JHPGL } & \text { Japanese High-Pressure Gas Law Test } \\ \text { LBV } & \text { laminar burning velocity } \\ \text { MBHP } & \text { mini breadboard heat pump } \\ \text { NBP } & \text { normal boiling point } \\ \text { NSD } & \text { no statistical difference }\end{array}$




\section{Nomenclature}

\section{Symbols}

$A_{\mathrm{c}} \quad$ cross-sectional area $\left(\mathrm{m}^{2}\right)$

$\mathrm{B}_{\text {nd }} \quad$ local Bond number $=\left(\rho_{1}-\rho_{\mathrm{v}}\right) \mathrm{g} e D_{\mathrm{h}} /(\sigma N),(-)$

Bo local boiling number $=\frac{q^{\prime \prime}}{G_{r} i_{f g}},(-)$

$c_{\mathrm{p}} \quad$ specific heat $\left(\mathrm{J} \mathrm{kg}^{-1} \mathrm{~K}^{-1}\right)$

$C \quad$ coefficients given in Eq. (3.4.5)

$D_{\text {e }} \quad$ equivalent inner diameter of smooth tube (m)

$D_{\mathrm{h}} \quad$ hydraulic diameter of micro-fin tube (m)

$e \quad$ fin height (mm)

g acceleration due to gravity $\left(\mathrm{m} / \mathrm{s}^{2}\right)$

$G \quad$ total mass velocity $\left(\mathrm{kg} \mathrm{m}^{-2} \mathrm{~s}^{-1}\right)$

$h_{2 \phi} \quad$ local two-phase heat-transfer coefficient $\left(\mathrm{W} \mathrm{m}^{-2} \mathrm{~K}^{-1}\right)$

$i_{\mathrm{fg}} \quad$ latent heat of vaporization $\left(\mathrm{J} \mathrm{kg}^{-1}\right)$

$k \quad$ refrigerant thermal conductivity $\left(\mathrm{W} \mathrm{m}^{-1} \mathrm{~K}^{-1}\right)$

$N \quad$ total number of microfins in cross section (-)

$\mathrm{Nu} \quad$ local Nusselt number based on $D_{\mathrm{h}}$

$\dot{m} \quad$ mass flow rate $\left(\mathrm{kg} \mathrm{s}^{-1}\right)$

$M_{\mathrm{w}} \quad$ molar mass $\left(\mathrm{g} \mathrm{mole}^{-1}\right)$

$p \quad$ wetted perimeter $(\mathrm{m})$

$P \quad$ local fluid pressure $(\mathrm{Pa})$

Pr liquid refrigerant Prandtl number $=\left.\frac{c_{p} \mu}{k}\right|_{r, l},(-)$

$Q_{\mathrm{vol}} \quad$ volumetric capacity $\left(\mathrm{kJ} \mathrm{m}^{-3}\right)$

$q^{\prime \prime} \quad$ local heat flux based on tube inner surface area $\left(\mathrm{W} \mathrm{m}^{-2}\right)$

$\operatorname{Re} \quad$ all liquid, refrigerant Reynolds number based on $D_{\mathrm{h}}, \quad \operatorname{Re}=\frac{G_{r} D_{h}}{\mu_{r, l}}$

$s \quad$ distance between fins ( $\mathrm{mm}$ )

$T$ temperature (K)

$t_{b} \quad$ bottom thickness of fin ( $\left.\mathrm{mm}\right)$

$t_{w} \quad$ tube wall thickness $(\mathrm{mm})$

$U \quad$ expanded relative uncertainty (\%)

$x_{\mathrm{q}} \quad$ thermodynamic mass quality (-)

$z \quad$ axial distance (m)

\section{Greek Symbols}

$\alpha \quad$ helix angle $\left({ }^{\circ}\right)$, learning rate

$\beta \quad$ fin angle $\left(^{\circ}\right)$

$\phi \quad$ fuel-air equivalence ratio

$\Delta L \quad$ incremental length (m)

$\Delta T_{\mathrm{s}} \quad \mathrm{T}_{\mathrm{s}}-T_{\mathrm{w}}(\mathrm{K})$ 


$$
\begin{array}{ll}
\mu & \text { viscosity }(\mathrm{Pa} \cdot \mathrm{s}) \\
v & \text { specific volume, } x_{\mathrm{q}} \nu_{\mathrm{v}}+\left(1-\mathrm{x}_{\mathrm{q}}\right) и\left(\mathrm{~m}^{3} \mathrm{~kg}^{-1}\right) \\
\rho & \text { density }\left(\mathrm{kg} \mathrm{m}^{-3}\right) \\
\sigma & \text { surface tension }\left(\mathrm{kg} \mathrm{s}^{-2}\right) \\
\omega & \text { overall chemical rate }\left(\mathrm{s}^{-1}\right)
\end{array}
$$

\section{Subscripts}

b bubble point

c critical condition

d dew point

f water

i inside, inlet

1 liquid

o outlet, exit

$\mathrm{p} \quad$ prediction, single component

psr perfectly stirred reactor

$r$ refrigerant

s saturated state

v vapor

w heat transfer surface

\section{Acknowledgements}

This work was supported by the Strategic Environmental Research and Development Program; Project WP19-1385: WP-2740 Follow-On: Low-GWP Alternative Refrigerant Blends for HFC-134a 


\section{Objective}

This work addresses the objectives of the Statement of Need number WPSON-17-20 "No/Low Global Warming Potential Alternatives to Ozone Depleting Refrigerants." It seeks to identify and experimentally demonstrate the performance and flammability behavior of three non-flammable refrigerant blends that can potentially replace HFC-134a in military environmental control units (ECUs). The selected fluids should have zero ozone depletion potential (ODP) and a global warming potential (GWP) less than that of HFC-134a. The selection criteria also include $\mathrm{COP}$, volumetric capacity $\left(\mathrm{Q}_{\mathrm{vol}}\right)$, and toxicity.

This work is a follow-on of the limited-scope project WP-2740, which screened over 100000 refrigerant blends using thermodynamic cycle simulation models and identified candidate HFC-134a replacements ${ }^{1}$. The current project narrows the pool of blend candidates down to three 'best' fluids, experimentally verifies their flammability behavior, and demonstrates their performance through tests in a military ECU in environmental chambers over a wide range of operating conditions.

To facilitate authoritative narrowing of the set of over 20 candidates to three 'best' blends for ECU testing, we carried out the following five tasks involving novel hydrofluoroolefin (HFO) fluids (Figure 1.0-1):

- Task 1: Fundamental measurements and modeling of thermophysical properties

- Task 2: Tests and modeling of flammability behavior

- Task 3: Preliminary performance tests in a mini-breadboard heat pump apparatus

- Task 4: Heat-transfer performance measurements and modeling

- Task 5: Selection of final blends for testing in military ECU

This Interim Report (Task 6) documents the results of Tasks 1 through 5. Tests of the selected three blends in the ECU will be carried out as Task 7 within the next stage of this project.

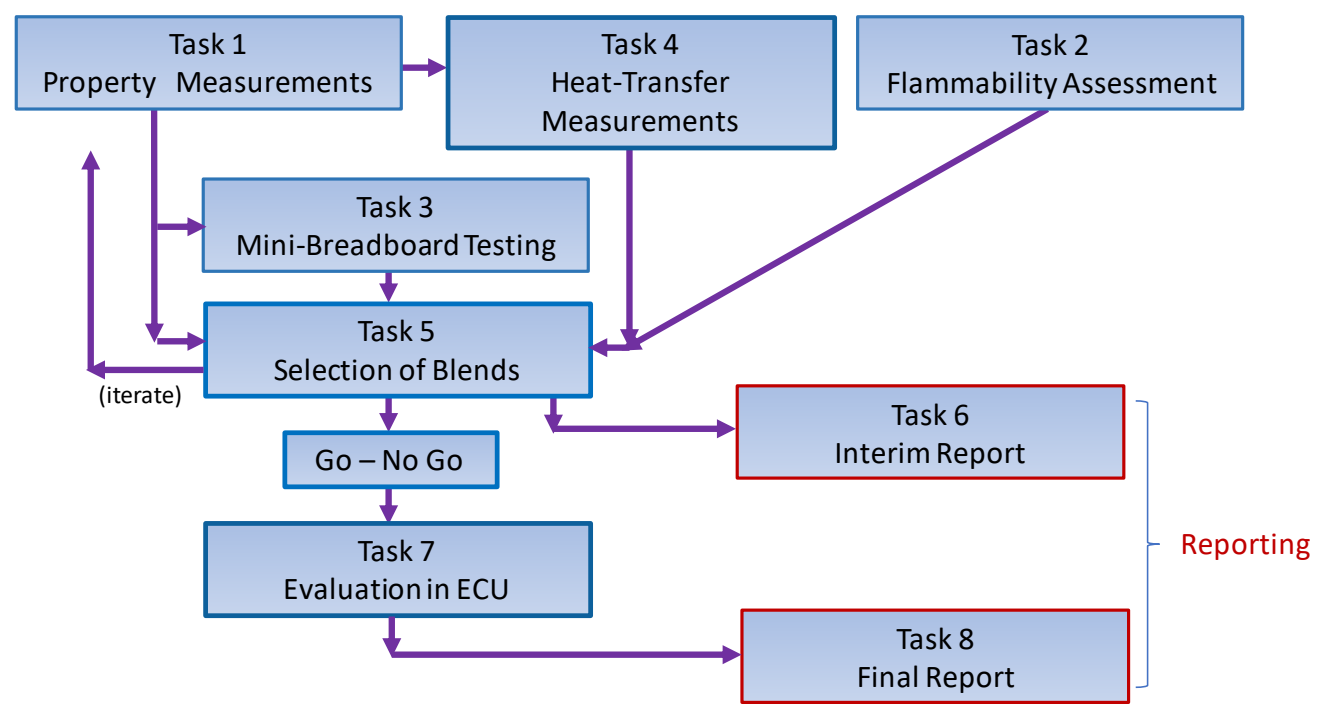

Figure 1.0-1. Project tasks 


\section{Technical Approach}

Prior work ${ }^{2}$ demonstrated that all single-component refrigerants that could serve-from the performance stand-point - as a replacement for HFC-134a are at least mildly flammable. For this reason, both the limited-scope project and the present work evaluate refrigerant binary and ternary blends to explore the possibility of formulating a non-flammable blend that would satisfy the requirements of military ECU systems.

The limited-scope project identified over 20 candidate low-GWP blends following an exhaustive search and evaluation of over 100000 two- and three-component blends among a slate of 13 single-component refrigerants, subsequently expanded to 14 refrigerants ${ }^{1,3}$. The limited-scope project was limited to computer simulations, while the present work will provide experimental results.

The candidate blends from the limited-scope project are listed here in Table 3.5-1. It was apparent that several of these candidate blends were very close in composition to existing compounds already included in ASHRAE Standard 34, so those similar blends, namely R450A, R-513A, and R-515B, were adopted.

Up to this point of the present project, we have selected three 'best' blends for ECU testing. The selection of the 'best' blends was based on (1) non-flammability; (2) minimum GWP; (3) maximum coefficient of performance (COP); and (4) the volumetric capacity $\left(Q_{\mathrm{vol}}\right)$ matching that of the baseline HFC-134a, where the $Q_{\text {vol }}$ of an air-conditioning system is a figure of merit related to the size of the compressor. The larger $Q_{\mathrm{vol}}$, the smaller the compressor needs to be for a given cooling capacity. This selection process was aided by laboratory measurements of refrigerant thermophysical properties, two-phase heat transfer performance, flammability behavior, and cycle performance tests in a mini-breadboard heat pump (laboratory vapor-compression test apparatus).

The next stage of the project will focus on testing of the three 'best' low-GWP blends and the baseline HFC-134a in a military ECU installed in environmental chambers over a wide range of operating conditions. These tests will involve 'soft optimization' of the ECU, which will entail a modification of the expansion device and optimization of refrigerant charge for each blend. Following these tests, we will extrapolate the ECU performance measurements with simulations using a detailed, first-principles-based system model to predict the performance potential of competing blends in a system with an optimized evaporator and condenser. 


\section{Results and Discussion}

\subsection{Task 1: Experimental Measurements of Blend Properties and Development of Mixture Equation of State}

\subsubsection{Purpose and Approach.}

A mixture equation of state (EOS) providing validated thermodynamic properties and separate transport property models are needed for conducting the MBHP tests (Task 3), refrigerant two-phase heat-transfer tests (Task 4), and ECU tests (Task 7). Updated properties will also be implemented in the NIST heat exchanger and ECU simulation models (Task 7).

The simulation of cycle performance, the analysis of laboratory measurements in equipment, and heat-transfer experiments in support of cycle simulation modeling require thermodynamic and transport properties of the blends. The thermodynamic properties are expressed in terms of an "equation of state," which is a mathematical model representing, in a consistent way, all of the thermodynamic properties of a pure fluid or blend. The properties of a refrigerant blend are given by a combination of the constituent pure fluids in the blend plus additional terms representing the mixture. The transport properties of thermal conductivity and viscosity are represented by separate models, also by combining pure-fluid models with mixture terms.

Any equation of state or transport property model (for a pure fluid or mixture) requires experimental data to fit adjustable parameters and validate its accuracy. While pure-fluid EOS are available and generally adequate for the new low-GWP refrigerants, this is not the case for refrigerant blends. This task has carried out the measurements necessary to define the mixture terms in the EOS and transport property models. These terms are expressed in terms of binary pairs of components; a mixture of the components $\mathrm{A}, \mathrm{B}$, and $\mathrm{C}$, for example, is expressed in terms of the binary pairs $\mathrm{A} / \mathrm{B}, \mathrm{A} / \mathrm{C}$, and $\mathrm{B} / \mathrm{C}$. Thus, the present measurements were carried out on binary mixtures, even though ternary (three-component) mixtures are also among the "best" blends. The mixture parameters used in the simulations in the limitedscope study were based on limited experimental data from the literature or, in some cases, were entirely predicted.

Measurements at two levels of effort were carried out. For three binary pairs (R-1234yf/134a, R-134a/1234ze(E), R-1234yf/1234ze(E)) that appear in multiple "best" blends, comprehensive measurements were carried out in Subtask 1a. These included vapor-liquid equilibrium (VLE) measurements; pressure-density-temperature-composition $(P, \rho, T, x)$ data in the single-phase and supercritical regions; speed of sound in the single-phase liquid region; viscosity in the single-phase liquid region; and thermal conductivity in the single-phase liquid region. Limited measurements (i.e., only vapor-liquid equilibrium) were carried out on additional binary pairs in Subtask 1b (R-125/1234yf, R-227ea/1234ze(E), R-152a/1234yf). For each blend, measurements were carried out at nominal compositions of $(0.33 / 0.67)$ and $(0.67 / 0.33)$ mole fraction. These measurements were designed to yield an optimal data set for the purposes of fitting mixture EOS and transport property models. The measurements are summarized in Table 3.1-1. The remainder of this section provides an overview of the measurements and modeling results, with details given in Appendix A.1. 
Table 3.1-1. Refrigerant blend properties measured in the current work.

\begin{tabular}{|c|c|c|c|c|}
\hline Blend & $\begin{array}{c}\text { Composition } \\
\text { (mole frac) }\end{array}$ & $\begin{array}{c}T \text {-range } \\
(\mathrm{K})\end{array}$ & $\begin{array}{c}P \text {-range } \\
\text { (MPa) }\end{array}$ & $\begin{array}{l}\text { Number } \\
\text { of points* }\end{array}$ \\
\hline \multicolumn{5}{|c|}{ Vapor-Liquid Equilibria (VLE) } \\
\hline R-1234yf/134a & $(0.320 / 0.680)$ & $270-360$ & $0.28-3.10$ & 10 \\
\hline R-1234yf/134a & $(0.647 / 0.353)$ & $270-360$ & $0.29-3.05$ & 10 \\
\hline R-134a/1234ze(E) & $(0.334 / 0.666)$ & $270-360$ & $0.22-2.61$ & 10 \\
\hline R-134a/1234ze(E) & $(0.663 / 0.337)$ & $270-360$ & $0.24-2.84$ & 10 \\
\hline R-1234yf/1234ze(E) & $(0.324 / 0.676)$ & $270-360$ & $0.23-2.56$ & 10 \\
\hline R-1234yf/1234ze(E) & $(0.638 / 0.362)$ & $270-360$ & $0.26-2.74$ & 10 \\
\hline R-125/1234yf & $(0.349 / 0.651)$ & $270-335$ & $0.39-2.21$ & 10 \\
\hline $\mathrm{R}-125 / 1234 \mathrm{yf}$ & $(0.664 / 0.336)$ & $270-335$ & $0.49-2.70$ & 10 \\
\hline R-227ea/1234ze(E) & $\dagger$ & $\dagger$ & $\dagger$ & $\dagger$ \\
\hline R-152a/1234yf & $\dagger$ & $\dagger$ & $\dagger$ & $\dagger$ \\
\hline \multicolumn{5}{|c|}{$(P, \rho, T, x)$ - Liquid and Vapor Phases and Supercritical States } \\
\hline R-1234yf/134a & $(0.3363 / 0.6637)$ & $230-400$ & $0.04-11.7$ & 124 \\
\hline$R-1234 y f / 134 a$ & $(0.6471 / 0.3529)$ & $230-400$ & $0.04-10.6$ & 105 \\
\hline R-134a/1234ze(E) & $(0.3325 / 0.6675)$ & $230-400$ & $0.04-20.5$ & 94 \\
\hline R-134a/1234ze(E) & $(0.6636 / 0.3364)$ & $230-400$ & $0.04-21.5$ & 81 \\
\hline R-1234yf/1234ze(E) & $(0.3358 / 0.6642)$ & $230-400$ & $0.04-11.4$ & 116 \\
\hline R-1234yf/1234ze(E) & $(0.6666 / 0.3334)$ & $230-400$ & $0.04-10.5$ & 109 \\
\hline \multicolumn{5}{|c|}{ Speed of Sound-Liquid Phase } \\
\hline R-1234yf/134a & $(0.3363 / 0.6637)$ & $230-345$ & $0.35-13.0$ & 78 \\
\hline$R-1234 y f / 134 a$ & $(0.6471 / 0.3529)$ & $235-310$ & $0.66-11.6$ & 40 \\
\hline R-134a/1234ze(E) & $(0.3292 / 0.6708)$ & $235-345$ & $0.53-51.4$ & 141 \\
\hline R-134a/1234ze(E) & $(0.6369 / 0.3631)$ & $230-345$ & $0.13-50.3$ & 163 \\
\hline R-1234yf/1234ze(E) & $(0.3358 / 0.6642)$ & $230-345$ & $0.26-11.3$ & 71 \\
\hline R-1234yf/1234ze(E) & $(0.6666 / 0.3334)$ & $230-335$ & $0.45-12.0$ & 60 \\
\hline \multicolumn{5}{|c|}{ Thermal Conductivity_Liquid Phase } \\
\hline R-1234yf/134a & $(0.320 / 0.680)$ & $200-340$ & $0.95-12.05$ & 379 \\
\hline $\mathrm{R}-1234 \mathrm{yf} / 134 \mathrm{a}$ & $(0.647 / 0.353)$ & $200-340$ & $1.03-12.00$ & 403 \\
\hline R-134a/1234ze(E) & $(0.334 / 0.666)$ & $200-340$ & $0.96-50.32$ & 350 \\
\hline R-134a/1234ze(E) & $(0.663 / 0.337)$ & $200-340$ & $0.97-50.14$ & 335 \\
\hline R-1234yf/1234ze(E) & $(0.323 / 0.677)$ & $200-340$ & $1.02-12.33$ & 352 \\
\hline R-1234yf/1234ze(E) & $(0.642 / 0.358)$ & $200-340$ & $0.89-12.10$ & 341 \\
\hline
\end{tabular}

*Distinct $(T, P)$ state points; multiple replicate measurements were made at each state point. ${ }^{\dagger}$ Measurements on two compositions of this blend were in progress as this report was being written. 


\subsubsection{Vapor-Liquid Equilibrium (VLE) Measurements.}

The measurement of vapor-liquid equilibrium is the mixture analog of vapor pressure for a pure component. VLE data are the most important type of data needed for fitting a mixture EOS. They indicate, for example, the departure from ideal behavior and the presence or absence of azeotropes. Measurements of the bubble-point pressure as a function of temperature and liquid-phase composition $(P, T, x)$ were made here. Conceptually, the measurement is very simple: load a liquid sample of known composition into a closed volume (i.e., "measuring cell"), bring the measuring cell to some temperature, and when the temperature has stabilized, measure the pressure. The VLE instrument and measurement details are described in Appendix A.1.1.

\subsubsection{Pressure-Density-Temperature $(P, \rho, T, x)$ Measurements.}

The present measurements utilized a two-sinker densimeter with a magnetic suspension coupling. This type of instrument applies the Archimedes (buoyancy) principle to provide an absolute determination of the density. This general type of instrument is described by Wagner and Kleinrahm, ${ }^{4}$ and the instrument used here is described in detail by McLinden and Lösch-Will, ${ }^{5}$ and only a brief description is given here. Two sinkers of nearly the same mass and same surface area, but very different volumes, were each weighed with a highprecision balance while they were immersed in the sample of unknown density. (The sinkers are shown in Figure A.1-3.) The fluid density $\rho$ is given by:

$$
=\frac{\left(\begin{array}{lll}
m_{1} & m_{2}
\end{array}\right)\left(\begin{array}{ll}
W_{1} & W_{2}
\end{array}\right)}{\left(\begin{array}{ll}
V_{1} & V_{2}
\end{array}\right)},
$$

where $m$ and $V$ are the mass and volume of the sinkers, $W$ are the balance readings, and the subscripts refer to the two sinkers. One sinker was made of tantalum $(m=60.094633 \mathrm{~g}$, $\left.V=3.60872 \mathrm{~cm}^{3}\right)$ and the other of titanium $\left(m=60.075386 \mathrm{~g}, V=13.315284 \mathrm{~cm}^{3}\right)$. A magnetic suspension coupling transmitted the gravity and buoyancy forces on the sinkers to the balance, thus isolating the fluid sample from the balance. The two-sinker method largely cancels out systematic errors in the weighing and from many other sources. Figure 3.1-1 illustrates the range of the measurements for one blend composition as an example.
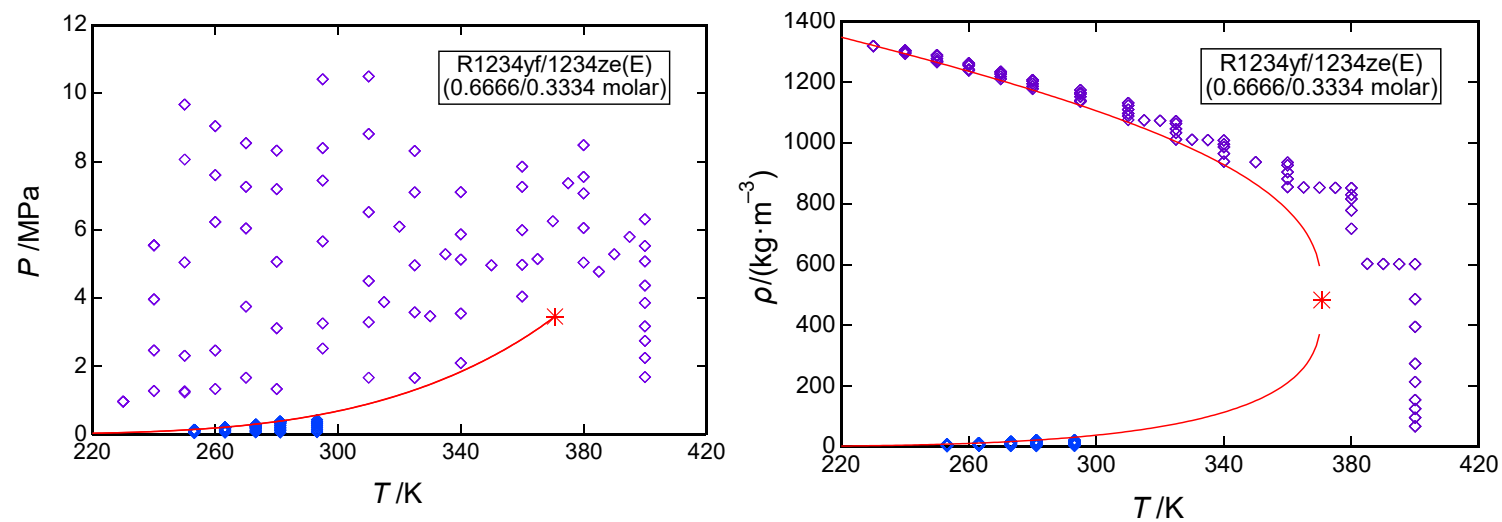

Figure 3.1-1. Measured $(P, \rho, T, x)$ points for the R-1234yf/1234ze(E) blend at a composition of (0.6666/0.3334) molar; left: pressure versus temperature; right: density versus temperature; $\diamond$, measured points; line, phase boundary; *, critical point. 


\subsubsection{Speed of Sound Measurements.}

The speed of sound is closely related to heat capacity, and either speed of sound or heat capacity data are important for obtaining the correct values for enthalpy and entropy, which are needed for cycle calculations. Enthalpy and entropy cannot be measured directly, and speed of sound can be measured much more accurately than heat capacity, which is the reason for including speed of sound.

The speed of sound was measured over a wide range of temperature and pressure in a dualpath, pulse-echo-type instrument. In this technique, a piezoelectric transducer of singlecrystal quartz is located within a sample volume of the test fluid. It is excited with a sinusoidal burst near the crystal resonance frequency, thus emitting ultrasonic pulses from each face of the crystal which travel through the fluid sample, reflect off planar surfaces at each end of the sample volume, and return to the transducer, which also serves as the detector. The difference in the arrival times of the echo signals give the speed of sound by

$$
w=\frac{2\left(L_{2}-L_{1}\right)}{\Delta t}
$$

where $w$ is the speed of sound, $L_{1}$ and $L_{2}$ are the path lengths, and $\Delta t$ is the time difference. The differential nature of this technique cancels end effects and improves the accuracy. The instrument, measurement sequence, and uncertainties are described in Appendix A.1.3.

An example of the measurements carried out is depicted

Figure 3.1-2. The measured data, as well as the relative, combined, expanded $(k=2)$ uncertainty in the speed of sound for each point, are reported in Appendix A.1.3. For both the $(P, \rho, T)$ and sound speed measurements, the maximum pressure was restricted to no more than $12 \mathrm{MPa}$ to avoid possible polymerization of HFO-1234yf.
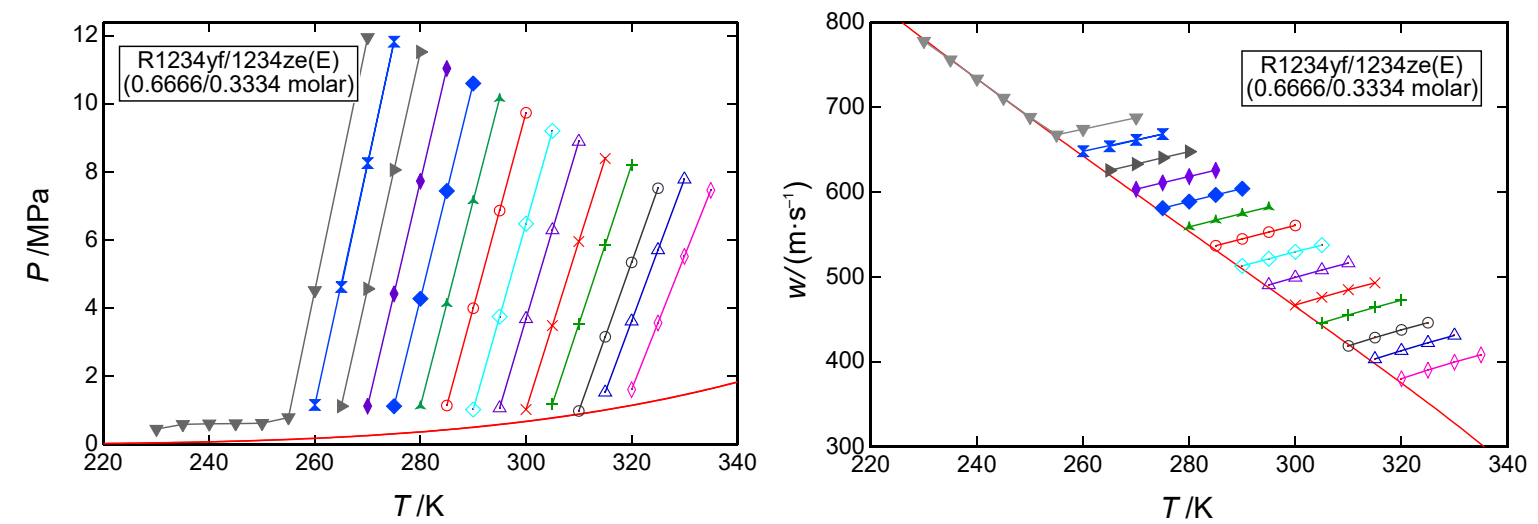

Figure 3.1-2. Measured speed of sound points for the R-1234yf/1234ze(E) blend at a composition of (0.6666/0.3334) mole fraction; left: pressure versus temperature; right: sound speed versus temperature; symbols are measured points and lines connect points along an isochore; red line is phase boundary. 


\subsubsection{Thermal Conductivity Measurements.}

Thermal conductivity cannot be calculated from an equation of state but is important for the analysis of heat-transfer behavior. Here, the thermal conductivity was measured with the transient hot-wire technique, whereby a voltage pulse lasting about $1 \mathrm{~s}$ is applied to a fine wire immersed in the fluid under test; this heats the surrounding fluid and the temperature rise is monitored (using the same wire, which also serves as a resistance thermometer) to extract thermal conductivity. The wire approximates a line source and the ideal temperature rise, $\Delta T_{\text {id, }}$ is given by

$$
\Delta T_{\mathrm{id}}=\frac{q}{4 \pi \lambda}\left[\ln (t)+\ln \left(\frac{4 a}{r_{0}{ }^{2} C}\right)\right]=\Delta T_{\mathrm{w}}+\sum_{i=1}^{10} \delta T_{i},
$$

where $q$ is the power applied per unit length, $t$ is the elapsed time, $r_{0}$ is the radius of the hot wire, $C=1.781 \ldots$ is the exponential of Euler's constant, $\lambda$ is the thermal conductivity of the fluid, and $a=\lambda\left(\rho C_{p}\right)$ is the thermal diffusivity of the fluid, where $\rho$ and $C_{p}$ are the fluid density and isobaric specific heat capacity. ${ }^{6}$ The form of Eq. (3.1-3) is that of a line, where the thermal conductivity can be found from the slope and the thermal diffusivity can be found from the intercept. The summation term accounts for deviations from the ideal model. These measurements provided the data necessary for development of a model for the thermal conductivity of refrigerant blends. The instrument, measurement sequence, and uncertainties are described in Appendix A.1.4; the measured data are also tabulated in Appendix A.1.4.

\subsubsection{Viscosity Measurements.}

Viscosity data cannot be calculated from an equation of state but are important for the analysis of heat-transfer behavior. Measurement of the viscosity of three refrigerant blends, at two compositions each, will be carried out in the second phase of the project.

\subsubsection{Model Development (Subtask 1c).}

The data measured in Subtasks 1a and $1 \mathrm{~b}$ were fitted to thermodynamic and transport property models with results provided in the form of files compatible with the NIST REFPROP database. Data are compared to existing models in REFPROP. The form of the model will be optimized and the data refit to the optimized model before the detailed system modeling of Task 7.

Mixture EOS for thermodynamic properties. The experimental data collected thus far have been compared against the default thermodynamic models implemented in NIST REFPROP 10.0. Significant differences were identified for the binary mixtures R-1234yf/1234ze(E) and R-1234yf/134a. For that reason, the interaction parameters for these blends were refit by applying the approach of Bell and Lemmon ${ }^{7}$. This approach uses evolutionary optimization to fit the mixture interaction parameters to minimize deviations in bubble-point pressure, as is detailed in Appendix A.1.6. The model implements the reducing functions (explained in Appendix A.1.6) of Kunz and Wagner"; and this is referred to as the "XR0" model in REFPROP. The result of the fitting is the set of new interaction parameters given in Table 3.1-2. The parameters for R-134a/1234ze(E) and R-125/R1234yf were not updated at this time. 
Table 3.1-2. Interaction parameters used in this work (note: the order of components is significant)

\begin{tabular}{lccccc}
\hline Binary Pair & $\beta_{T, i j}$ & $\gamma_{T, i j}$ & $\beta_{V, i j}$ & $\gamma_{V, i j}$ & $F_{i j}$ \\
\hline R-1234yf/1234ze(E)* & 1.000555 & 0.992815 & 1.0 & 1.0 & 0.0 \\
R-1234yf/134a* $^{*}$ & 0.999557 & 0.986956 & 1.0 & 1.0 & 0.0 \\
R-134a/1234ze(E) & 1.0 & 0.992 & 1.0 & 1.0 & 0.0 \\
R-125/1234yf $^{\dagger}$ & 1.0 & 0.999 & 1.0 & 1.0 & 0.0 \\
\hline
\end{tabular}

*updated values for the mixture interaction parameters

${ }^{\dagger}$ mixture interaction parameters from REFPROP 10.0

Deviations of the data with the updated models are shown in Figure 3.1-3. By tuning the interaction parameters, the deviations in bubble-point pressure (i.e., VLE data) have been reduced to within or near their experimental uncertainty. The density data are also well represented, except for densities in the critical region, where the uncertainties are larger due to the propagation of uncertainty in pressure measurement through to the combined expanded $(k=2)$ uncertainty. On the other hand, the adjustment of the interaction parameters to reduce deviations in bubble-point pressure did have a negative effect on the reproduction of the speed of sound, although all the speed of sound deviations are within $2 \%$ with the updated parameters, and most are within $1 \%$. Considering the combined expanded uncertainty of the speed of sound measurements is approximately $0.07 \%$, there remains an opportunity to update the mixture models to yield a better representation of the caloric properties by better reproduction of the speed of sound data. This is perhaps not a surprising result because the current mixture model for refrigerants, that of Lemmon and Jacobsen ${ }^{9}$, was developed in the early 2000s with virtually no speed of sound data as input. This is a target for the second phase of the project in which we will generate reference thermodynamic models, with the goal being to reproduce all experimental data measured in this project to within their experimental uncertainties.

Thermal conductivity model. We have applied the modified entropy scaling approach to model the measured thermal conductivity data. Entropy scaling is a relatively new approach for the modeling of transport properties and is based on the idea that entropy (a thermodynamic property), when scaled in an appropriate way, is a measure of the structure of the fluid phase. Thus, transport properties can be expressed in terms of this scaled entropy, rather than as a function of, for example, the temperature and density. This approach connects the transport properties (e.g., thermal conductivity and viscosity) to the thermodynamic properties (which are represented by an equation of state). The power of entropy scaling is that it allows an accurate representation of the transport properties with far fewer experimental data needed as input to the model compared to traditional models.

The thermal conductivity $\lambda$ is non-dimensionalized to the plus-scaled thermal conductivity $\lambda^{+}$ as defined by

$$
\lambda^{+}=\lambda /\left(k_{B}\left(\rho N_{A}\right)^{2 / 3} \sqrt{R T / M}\right) \times\left(s^{+}\right)^{2 / 3}
$$



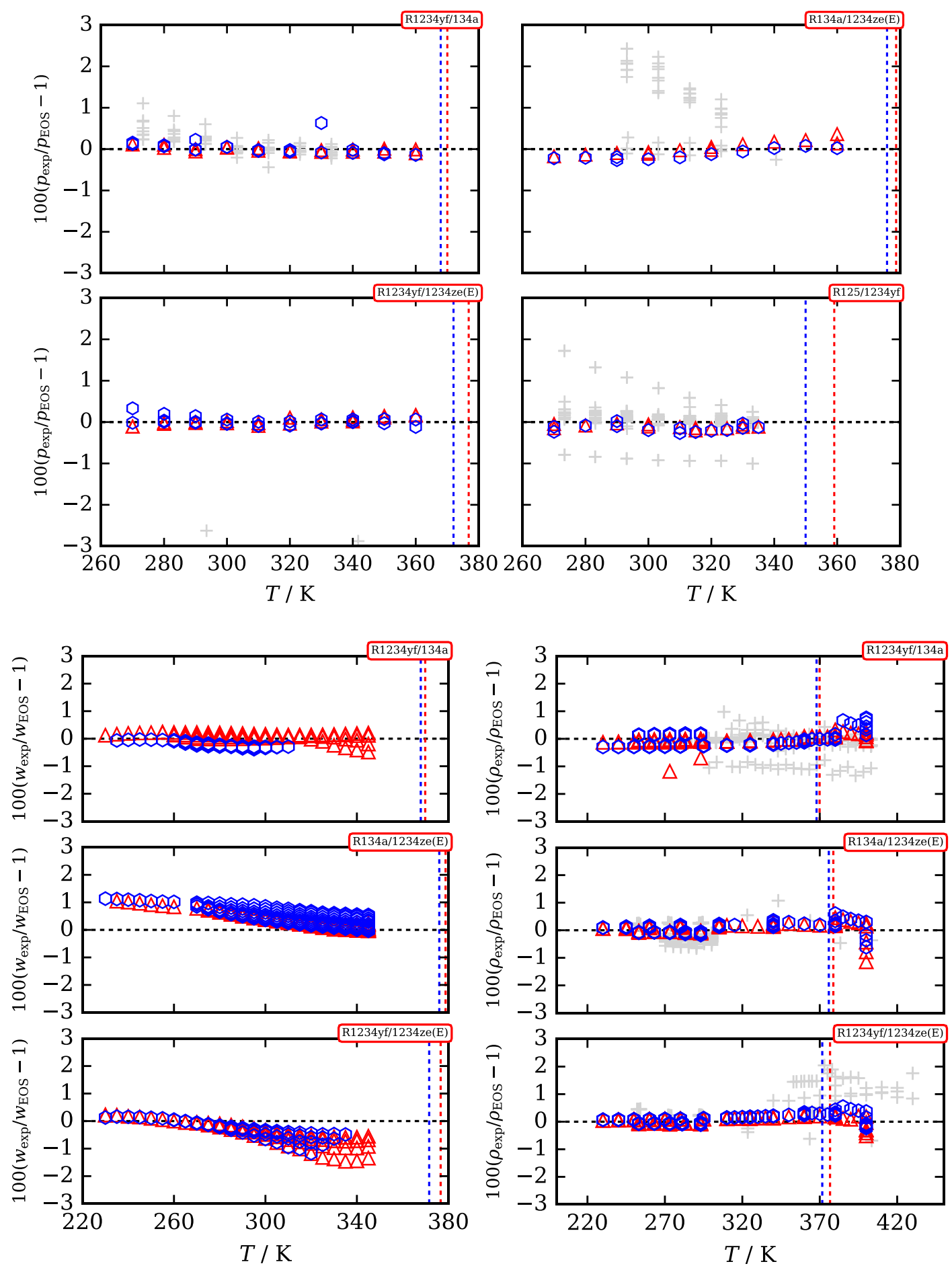

Figure 3.1-3. Deviation plots for the measured experimental data with the mixture EOS using the parameters from Table 3.1-2. The different plot symbols indicate the two compositions measured here; the vertical lines indicate the mixture critical temperature. Literature data are shown as light grey + markers (some literature data have deviations outside the range of the figures). 
$\lambda^{+}$is a function of the reduced scaled residual entropy $s^{+}$, with a factor including the molar density $\rho$, Boltzmann constant $k_{\mathrm{B}}$, Avogadro's constant $N_{A}$, temperature $T$, and molar mass $M$. All dimensional quantities are expressed in base-SI units ( $\mathrm{kg}, \mathrm{m}, \mathrm{Pa}$, etc.). The origins of this scaling are described in Appendix A.1-6.

In these scaled coordinates, the experimental mixture thermal conductivity data fall very nearly along a single curve, as shown in Figure 3.1-4. The curve is given by a simple polynomial fitted to the experimental data by unweighted linear least squares:

$$
\lambda^{+}=\sum_{i} c_{i}\left(s^{+}\right)^{i}
$$

where the coefficients $c_{i}$ are given in Table 3.1-3, and the deviations are shown in Figure 3.1-4.(b). The average absolute deviation is $1.32 \%$ and the central 95.5 percentiles of the signed relative errors (approximately equivalent to a $95 \%$ confidence interval) span the range $-2.82 \%$ to $2.98 \%$. This quality of fit is rather remarkable given that a total of six mixtures formed of three different components are collapsed to a single curve with no empirical tuning parameters. While this interim correlation was fitted to only liquid phase data, with a dilute-gas model and a critical enhancement model, its range of validity can be extended to the entire phase diagram, from dilute gas to the melting line.

Table 3.1-3. Coefficients for entropy scaling of thermal conductivity data (Eq. 3.1-5).

\begin{tabular}{lc}
\hline $\mathrm{i}$ & $\mathrm{c}_{\mathrm{i}}$ \\
\hline 0 & 15.19495 \\
1 & -6.29123 \\
2 & 2.74329 \\
3 & -0.12635 \\
\hline
\end{tabular}
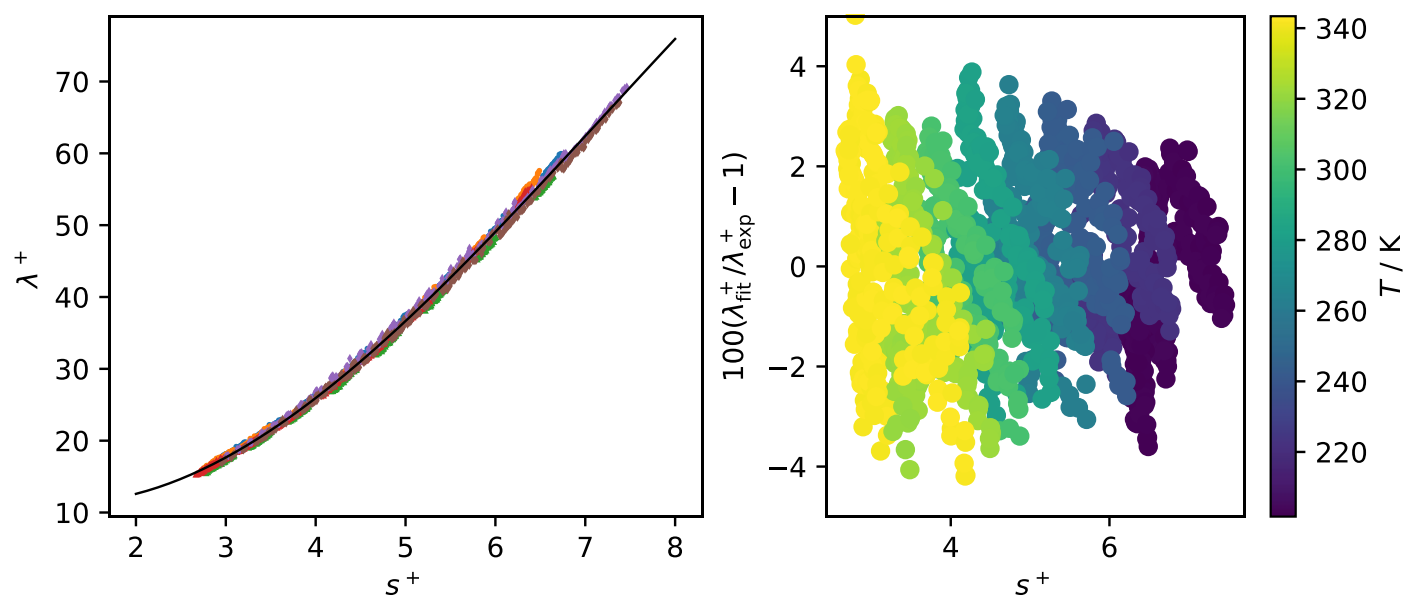

Figure 3.1-4. Entropy scaling model for the thermal conductivity of the three blends studied here; (a) plus-scaled thermal conductivity $\lambda+$ as a function of reduced scaled residual entropy $s+$, the different symbols denote the different measured blends; (b) relative deviations in $\lambda+$. 


\section{2. $\quad$ Task 2: Flammability Testing}

3.2.1. Introduction

It is essential that any low-GWP replacement for HFC-134a be non-flammable. This is challenging, however, since for molecules containing only hydrogen, fluorine, and carbon, there is a trade-off between GWP and flammability. The common changes to the molecules (adding hydrogen atoms or double bonds) to make them more reactive in the troposphere and hence lower their atmospheric lifetime (which lowers GWP), also makes them more flammable. Thus, one desires to make the molecules, or mixtures of compounds, have the lowest GWP possible while still maintaining non-flammable behavior. A further challenge arises, however, in that flammable behavior is not a distinct boundary, but depends upon the environment to which the refrigerant is exposed. As described by Williams ${ }^{10}$, flame stabilization requires that the characteristic chemical reaction rate be fast enough to keep up with the flow/transport field in which the refrigerant is reacting. For compounds that are clearly flammable or non-flammable, for example methane or $\mathrm{CO}_{2}$, their behavior in air is consistent among common configurations. But for compounds of intermediate flammability, for example mixtures of $\mathrm{CO}_{2}$ and $\mathrm{CH}_{4}$, or pure $\mathrm{HFO}-1234 \mathrm{yf}$, their behavior will be very sensitive to the flame/fire configuration. For example, in recent full-scale tests in a $50 \mathrm{~m}^{3}$ module ${ }^{11}$, stable flames of HFO-1234yf and air could not be initiated with a high-voltage spark ignition system typical of that used in the small-scale E681 test ${ }^{12}$. In contrast, using a glow-plug ignition source, turbulent flames of HFO-1234yf and air in an HVAC duct were achieved by Papas et al. ${ }^{13}$, with turbulent flame speeds much higher than previously reported laminar burning velocities.

There are two goals of the present refrigerant flammability work. The first is to assess experimentally the flammability of the candidate blends predicted to be non-flammable (as determined by ASHRAE Standard $34{ }^{12}$ ) in the previous limited-scope project. In the preliminary work, an empirical model of flammability based on the adiabatic flame temperature and the fluorine to hydrogen ratio of the reactants was used to create a flammability index ${ }^{14}$ and rank a list of candidate blends with regard to their flammability. All of the candidate blends selected for further study in the MBHP or ECU were predicted to be non-flammable. Nonetheless, it is essential that the candidate blends be tested in experiments to verify that the predictions were accurate. It is expected, however, that the fire threats in the DOD applications will be much more aggressive than in typical HVAC applications, and a more conservative flammability test would be desirable.

The second goal of the present work is to use the experimental flammability test results, together with flame modeling, to predict, for a given blend, if it will be flammable in the fire threats of interest to the DOD. Researchers at the Army Research Laboratory (ARL) have developed a representative scenario, in which an incendiary projectile impacts a coil filled with refrigerant. In such a test, ignition sources are abundant, and it is required that the resulting refrigerant-air cloud not support a self-propagating flame. Predicting the behavior of candidate lower-GWP refrigerant blends in that test is challenging. Of course, all candidate blends could be tested at full-scale, but this would be prohibitively expensive and time consuming. It is desired to predict the full-scale behavior based on laboratory-scale test results. While challenging, such a prediction is not unprecedented. For example, the behavior 
of HFC fire suppressant agents in the FAA Aerosol Can Test ${ }^{15}$ has many similarities as will be discussed below.

The approach for flammability assessment is as follows. First, the flammability of the candidate blends must be experimentally tested. Since the empirical model for refrigerant flammability in the limited-scope project was developed based upon the data in the ASHRAE Standard 34 database, the modified ASTM E681 specified in Standard 34 is used to experimentally verify the predicted performance of the candidate blends. Next, a more stringent test is applied. For this purpose we adopt a modified version of the Japanese HighPressure Gas Law (JHPGL) test ${ }^{16}$, as described below. Development of the kinetic model of combustion of the refrigerant-air flames is discussed, and the model is used to interpret the experimental data available to date. Finally, an approach for connecting the small-scale tests to the full-scale live-fire tests to be conducted at ARL is described, with the goal of properly interpreting those results and minimizing the number of tests necessary to ensure nonflammable behavior of the selected blends.

\subsubsection{E681 tests and Japanese High-Pressure Gas Law tests}

E681: Experimental Results. For HVAC applications, the flammability of a refrigerant is assessed via ANSI/ASHRAE Standard $34^{17}$ or the very similar ISO Standard $817{ }^{18}$. A flammability rating of Class " 1 " to " 3 " is assigned, based on its heat of combustion, lower flammability limit, and laminar burning velocity. In the ASHRAE standard, Class 3 fluids, termed "higher flammability," exhibit flame propagation at $60{ }^{\circ} \mathrm{C}$ and $101.3 \mathrm{kPa}$ and have a heat of combustion greater than $19 \mathrm{MJ} / \mathrm{kg}$ or a lower flammability limit (LFL) less than $0.10 \mathrm{~kg} / \mathrm{m}^{3}$. Class 2 fluids, "lower flammability" have a heat of combustion less than $19 \mathrm{MJ} / \mathrm{kg}$ and an LFL greater than $0.10 \mathrm{~kg} / \mathrm{m}^{3}$. Class 1 fluids exhibit "no flame propagation" when tested at $60{ }^{\circ} \mathrm{C}$ and $101.3 \mathrm{kPa}$. Class "2L" fluids also have a maximum burning velocity less than $10 \mathrm{~cm} / \mathrm{s}$. For the present discussion, we focus on the distinction between Class 1 and Class 2L.

Flame propagation and the lower flammability limit in ASHRAE Standard 34 are determined by the test method specified in ASTM E681 ${ }^{19}$, with slight modifications. A schematic of the E681 test vessel is shown in Figure 3.2-1. In the test, the refrigerant and air are introduced into a $12 \mathrm{~L}$ glass sphere, which is closed by a spring-loaded stopper at the top. A $15 \mathrm{kV} \mathrm{AC}$ source provides a spark (spark duration $0.4 \mathrm{~s}$ ) to tungsten electrodes ( $1 \mathrm{~mm}$ diameter, $6.4 \mathrm{~mm}$ gap) located 1/3 diameter from the bottom of the vessel. If a flame forms and extends upwards and outwards from the spark to the walls of the flask and subtends an angle equal to or greater than $90^{\circ}$ as measured from the point of ignition, there is "flame propagation." Tests are conducted at $60{ }^{\circ} \mathrm{C}$ with air at $50 \%$ relative humidity (r.h. $)$ at $23{ }^{\circ} \mathrm{C}(0.0088 \mathrm{~g}$ $\mathrm{H}_{2} \mathrm{O} / \mathrm{g}$ air). 

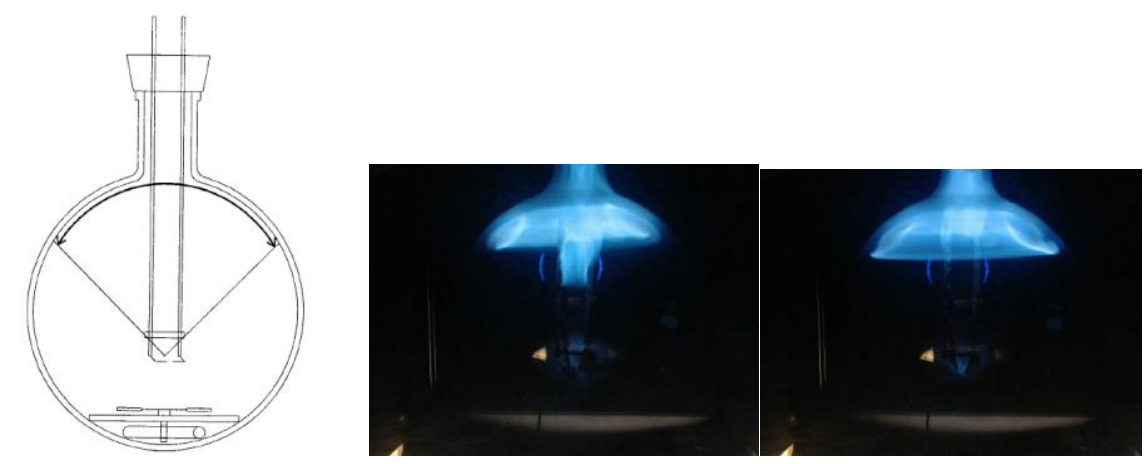

Figure 3.2-1. Left image: schematic of ASTM 681 test vessel; Right two images: visual images from HFC-32/HFC-134a blend ( $0.35 / 0.65$ mass fraction) below and above the lean flammability limit ${ }^{20,21}$.

The ASHRAE Standard 34/ASTM E681 tests for the present work were performed by an outside testing laboratory under contract to NIST. This contractor was selected because they have extensive experience with the test ${ }^{21}$ and have provided a substantial number of the data in the ASHRAE Standard 34 database. Considering that there is significant variability in the test approaches that can affect the quantitative results ${ }^{21,22}$, it was useful to select a contractor likely to provide a test approach consistent with the existing database. This was important since the present test results are being compared to flammability predictions from our earlier work ${ }^{1,14}$, which was developed based on the existing ASHRAE Standard 34 database.

It was originally expected that all mixtures selected for further study would need to be tested by the outside contractor. However, over the course of the project, it was apparent that several of the candidate blends were very close in composition to existing compounds for which applications to ASHRAE 34 had recently been made, so those similar blends (R-450A, R-513A, and R-515B) were adopted. A side benefit is that they would not need to be tested again via the contracted E681 experiments, freeing up some of those tests for other compounds of interest.

The results of the contracted E681 tests are listed in Section A.2.1 of Appendix A. Table 3.21 below summarizes the results of the E681 tests and lists the existing flammability ratings ${ }^{14}$ for R-513A, R-450A, and R-515B from the ASHRAE Standard 34 database $\left(P_{\max }\right.$ and $\omega_{p s r}$ are discussed below). All of the candidate blends are flammability Class 1 . The last three mixtures, blends of HFO-1234yf and HFC-134a, were tested at increasing concentration of HFC-134a until a Class 1 rating was achieved. These tests will be used to connect E681 experiments, JHPGL experiments, and simulations to full-scale results, as discussed below. 
Table 3.2-1. Modeling and test results for candidate blends (reactants at $296 \mathrm{~K}, 101 \mathrm{kPa}$, and $50 \%$ r.h.).

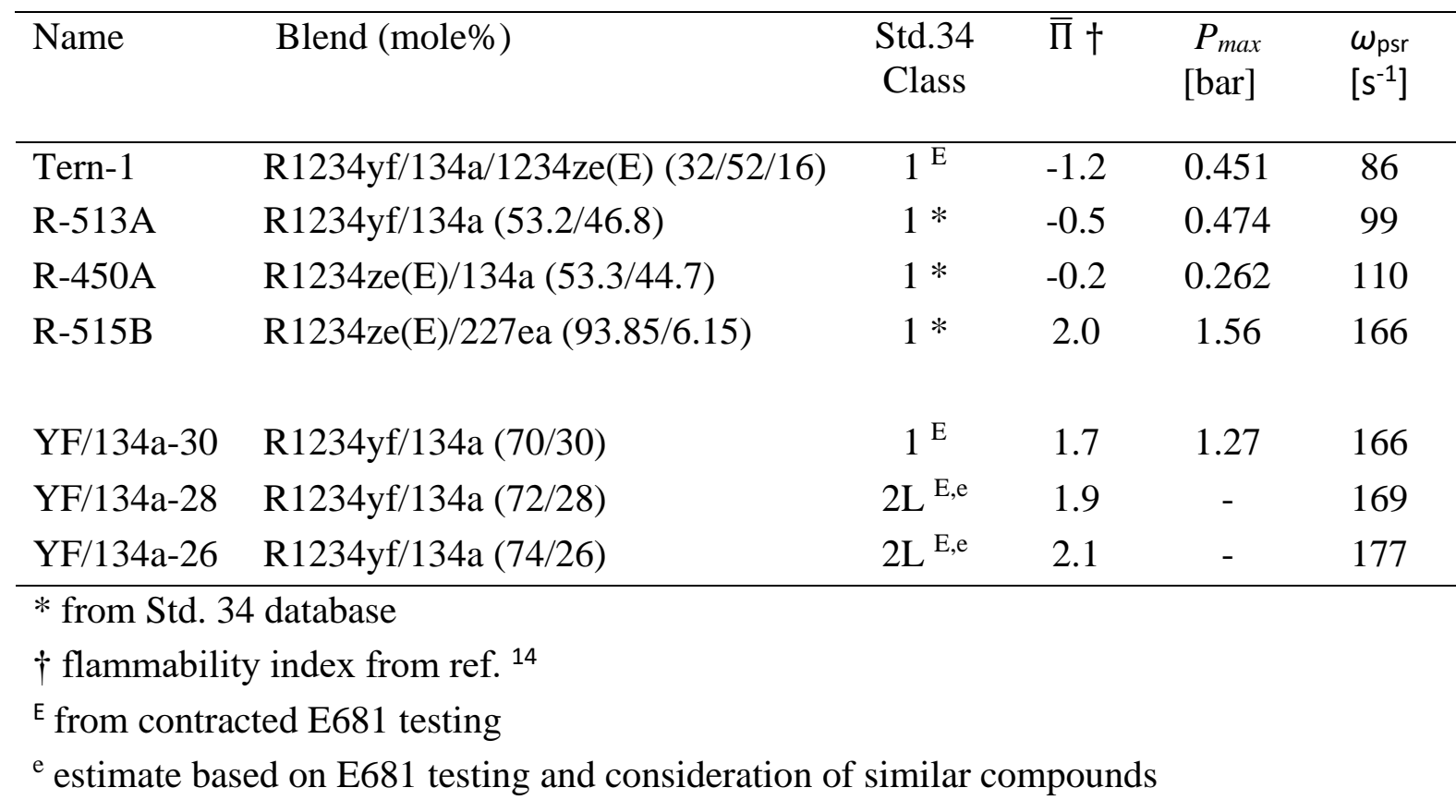

E681: Interpretation of E681 Results in Regard to Large-Scale Behavior. The E681 test, as modified by ASHRAE Standard 34, is widely used in the HVAC industry and is believed to be sufficiently conservative to provide safe application of refrigerants in those settings. It may not, however, be appropriate for the much more stringent non-flammable requirements of DOD applications. As described above, flammability is a continuum, and only becomes a discrete rating when some criterion is applied. As an example, the $90^{\circ}$ flame angle criterion is used in the ASHRAE Standard 34 to distinguish between "flame propagating" and "nonflame propagating." This is illustrated in Figure 3.2-1, in which the left and right flame images (with flame angles of $85^{\circ}$ and $95^{\circ}$, respectively) are for refrigerant concentrations in air that are just below and above the lean flammability limit. To an observer, these flames might both appear to be "flammable," and hence the $90^{\circ}$ criterion may not be appropriate for more energetic, turbulent-ignition scenarios such as in the Army Research Laboratory ARL live-fire tests.

The selection of the $90^{\circ}$ criterion is informative for the present discussion. Richard and coworkers ${ }^{23}$ conducted experiments in larger, $200 \mathrm{~L}$ vessels in which the walls did not constrain the effects of buoyancy on the flame propagation. They found that some flames propagated and consumed all the fuel in the vessel while others self-extinguished and failed to propagate. The successful flame propagation in the $200 \mathrm{~L}$ vessel correlated with those flames in the $12 \mathrm{~L}$ vessel that had a flame angle of $90^{\circ}$ or greater, validating the $90^{\circ}$ criterion in the ASHRAE Standard 34/E681 test. Note that as an additional measure of safety, the ASHRAE Standard 34 test is conducted with gases at $60^{\circ} \mathrm{C}$, i.e., a more flammable condition, so that a non-flammable rating obtained at the test condition is conservative. Nonetheless, concerns have been raised with regard to the $90^{\circ}$ criterion. For example, only two refrigerant blends were used, i.e., R-152a/134a and R-152a/125, and it is unclear if the 
behavior will be the same for different chemical classes of refrigerants, e.g., HFOs vs HFCs. Also, the stretched burning velocity and critical flame radius for flame propagation are known to depend on the Lewis number of the mixture ${ }^{24,25}$, which can vary for different blend components.

The challenge in the present work is to find a relevant flammability criterion in small-scale tests that properly captures the important flame dynamics in the full-scale, live-fire tests of interest to the Department of Defense, which is characterized by strong, multiple ignition sites (due to projectile impact), heated product gases from ancillary combustion (from the pyrotechnic charge) and shock-induced heating, and turbulence. The next section presents the rational for selecting the Japanese High-Pressure Gas Law (JHPGL) test, and some experimental results from it with the candidate blends and R-1234yf/134a mixtures.

Japanese High-Pressure Gas Law Test: Relevance. A modified version of the Japanese High-Pressure Gas Law (JHPGL) test (documented in the appendix of ref. ${ }^{16}$ ) has been selected to provide a more stringent, reduced-scale flammability test for refrigerants exposed to live-fire tests at full scale. The experiment uses a $2 \mathrm{~L}$ stainless steel spherical pressure vessel into which the premixed gases are introduced using the partial pressure method. A thin platinum wire energized by a continuous 100 VAC source provides ignition. The explosion pressure $P_{\max }$ (i.e., the maximum pressure in the vessel for any value of fuel-air equivalence ratio $\phi$ ) provides the metric for flammability. The experiment is described in detail in previous work ${ }^{16,26-28}$ and in Section A.2.2 of Appendix A, and has been used to understand HFC refrigerant flammability ${ }^{16,26}$ as well as HFC fire-suppressant performance in aircraft fire-suppression applications ${ }^{27,28}$. Modifications from the original standard are the use of pressure rather than temperature as the flammability metric, and a modified ignitor configuration to allow insertion through a single port.

The test indicates wider flammability limits than the ASHRAE Standard 34 E681 test for the following reasons. The exploding platinum wire sprays droplets of molten platinum through the chamber ${ }^{29}$, providing numerous ignition sites by catalytic surfaces, and the turbulent flow field accelerates the flame speed and minimizes stretch-induced extinction of the nascent flames ${ }^{30}$ at early times. The constant volume combustion chamber also provides compressive heating, which increases reactivity. In previous work, flammability in the JHPGL test was shown to accurately predict flammability of HFC/hydrocarbon/air mixtures in the very intense conditions of the FAA Aerosol Can Test (FAA-ACT). In that test, a $10 \mathrm{~m}^{3}$ pressure vessel is used to mimic the inside of an airliner cargo-bay, in which an aerosol spray can explodes due to the heat of a cargo-bay fire. In the FAA-ACT, a fast-acting valve releases the contents of the simulated aerosol can (454 $\mathrm{g}$ of propane, ethanol, and water) across a $15 \mathrm{kV}$ continuous AC spark ignitor, into the chamber which has been prefilled with a mixture of air and an HFC fire suppressant (e.g., HFC-125, 2-BTP, Novec 1230, etc.). The contents of the aerosol can form an intense propane/ethanol/water turbulent spray flame at the ignitor location, and the question is whether the flame propagates into the air/HFC endgases in the chamber. The pressure rise in the chamber due to reaction of the aerosol can contents (and sometimes HFC suppressant) creates a temperature rise of $100{ }^{\circ} \mathrm{C}$ to $250{ }^{\circ} \mathrm{C}$. The similarities with the ARL live-fire refrigerant-coil test include: strong ignition source, temperature rise due to compressive heating, reactive intermediates supplied by strong 
combustion of an adjacent flame, and basically the same type of reactants (HFCs, HFOs, etc. mixed with air) in the end gases, which may or may not be ignited. The FAA-ACT also provides extra water (i.e., mixed with the fuel), enhancing the reactivity of the HFC compounds. The fact that the JHPGL test could accurately predict which fire suppressants (and blends) agents would combust in the end-gases of the FAA-ACT ${ }^{28}$ gives confidence that it is a good small scale test for predicting the behavior of similar compounds in the ARL live-fire refrigerant tests.

Japanese High-Pressure Gas Law Test: Experimental Results. The JHPGL as used provides the peak pressure rise in the vessel as a function of blend volume fraction in the air. Figure 3.2-2 shows, for the four candidate blends, the measured peak pressure as a function of refrigerant volume fraction in moist (50\% r.h.) air (lower curves) together with the calculated 31 equilibrium explosion pressures for the same mixtures. Equilibrium values are about 10 bar for all blends, varying about $1 \%$ between the candidate blends, and by about $1 \%$ for varying water vapor in the air ( $0 \%$ to $100 \%$ r.h.). The equilibrium value assumes complete reaction to most stable products with no heat losses. For typical fast hydrocarbon flames, $90 \%$ of the equilibrium value can be attained ${ }^{27}$. For slower flames, the pressure rise is a smaller fraction of equilibrium, for example about $50 \%$ for HFO-1234yf. Fusing of the Pt wire causes a pressure rise of $0.033 \mathrm{bar}+/-0.013 \mathrm{bar}^{16}$. Thus, the candidate blends have pressure rises of $2 \%$ to $13 \%$ of the equilibrium values.

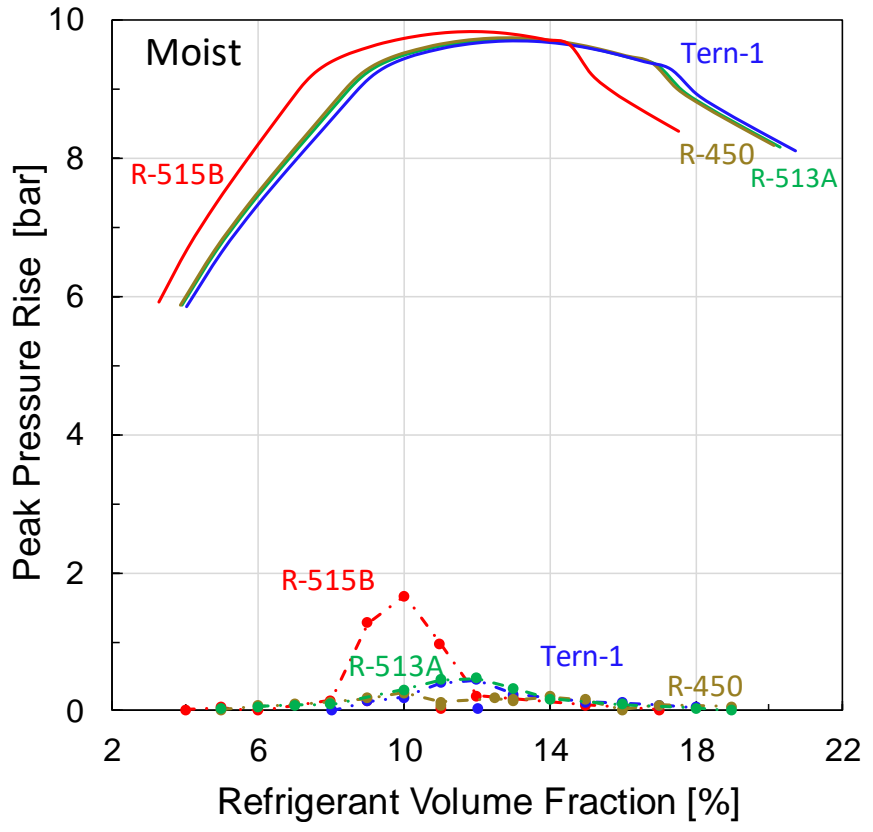

Figure 3.2-2. Equilibrium explosion pressure of candidate blends (Tern-1, R-513A, R-450A, and R-515B) together with experimental results in the JHPGL test as a function of blend volume fraction in air (50\% r.h.).

The experimentally observed peak pressure rise for the four candidate blends is given in Section A.2.2 of Appendix A and shown graphically in Figure 3.2-3 for dry and moist air. Focusing on the moist air results (right frame), the peak pressure for the four agents: R-450A, Tern-1, and R-513A is low $(0.262,0.451$, and 0.474) bar, but higher for R-515B, about 
1.6 bar. Details of the results for individual blends are shown in Figure 3.2-4 (note difference in scales). While these data may appear noisy, it is important to keep in mind that these are very low pressure rises, at the lower end of the dynamic range of the piezo-electric dynamic pressure transducer. Also, the stochastic nature of the exploding-wire ignition system creates variability in the results. The individual results in Figure 3.2-4 illustrate the effects of water vapor, which are small for R-450A and R-515B, and larger for R-513A and the Tern-1 blend.
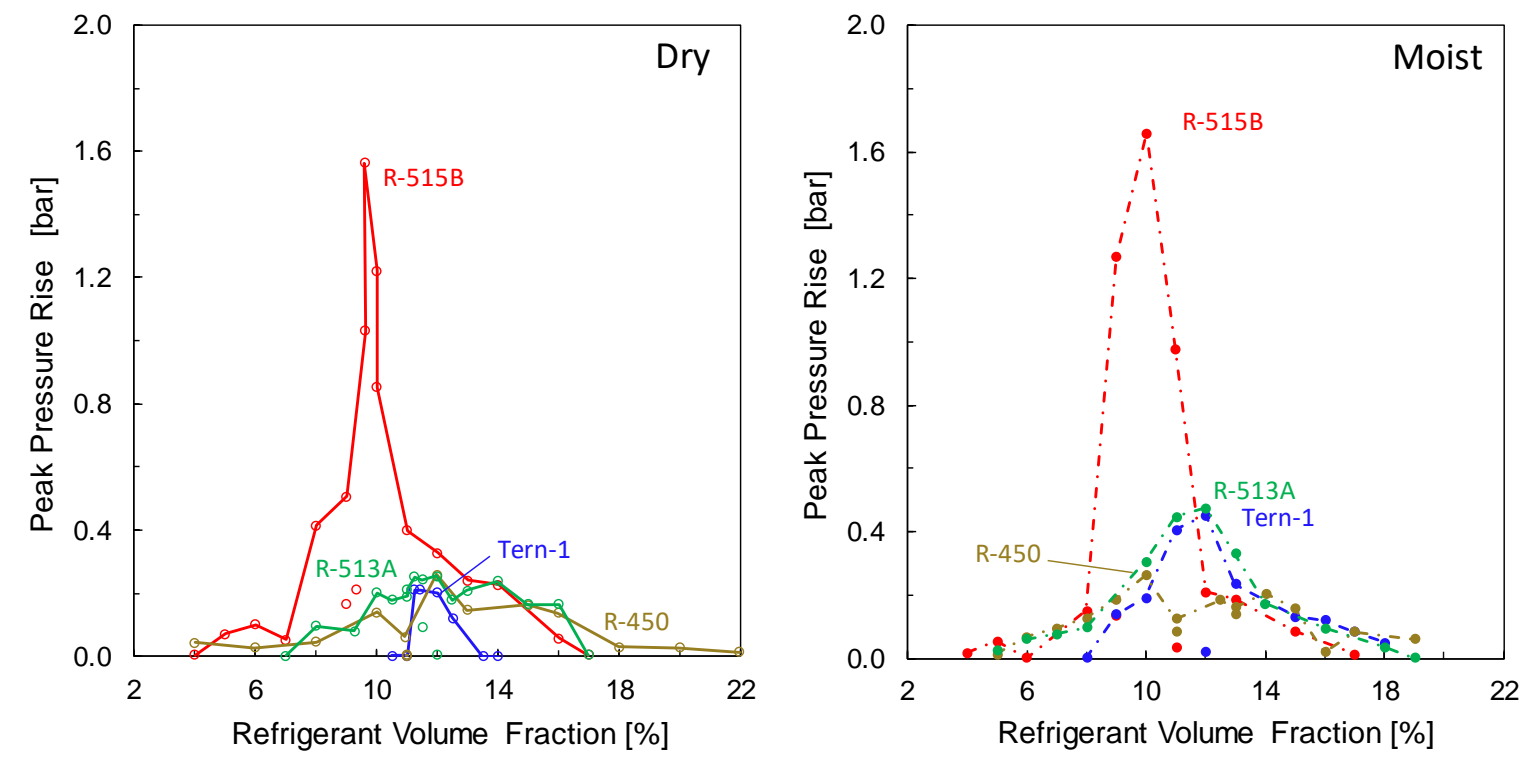

Figure 3.2-3. Explosion pressure of candidate blends in JHPGL test as a function of blend volume fraction in air (left frame: dry air; right, moist).

While the results of the tests for the candidate blends are useful and illuminating, there is value in conducting tests in which the blend components are more systematically varied. To do this, we considered a binary blend of HFC-134a with HFO-1234yf, with increasing mole fraction of the less-flammable component (HFC-134a) up to $100 \%$. Figure 3.2-5 shows the JHPGL explosion pressure as a function of blend volume fraction in the air; each curve refers to a particular HFC-134a volume fraction in the blend (left frame: dry air; right frame: moist air, $50 \%$ r.h.). Figure 3.2-6 shows the peak values from Figure 3.2-5 as a function of the volume fraction of HFC-134a in the blend. For pure HFO-1234yf flames, moist flames have about $25 \%$ higher peak pressure than do dry flames. For both moist and dry air, the peak pressure drops off steadily until about 50/50 volume fraction, after which the reduction in peak pressure with increasing HFC-134a is small. That is, after about $60 \%$ HFC-134a volume fraction, there appears to be no additional benefit of adding this component to HFO$1234 y f$, in this experimental configuration. 

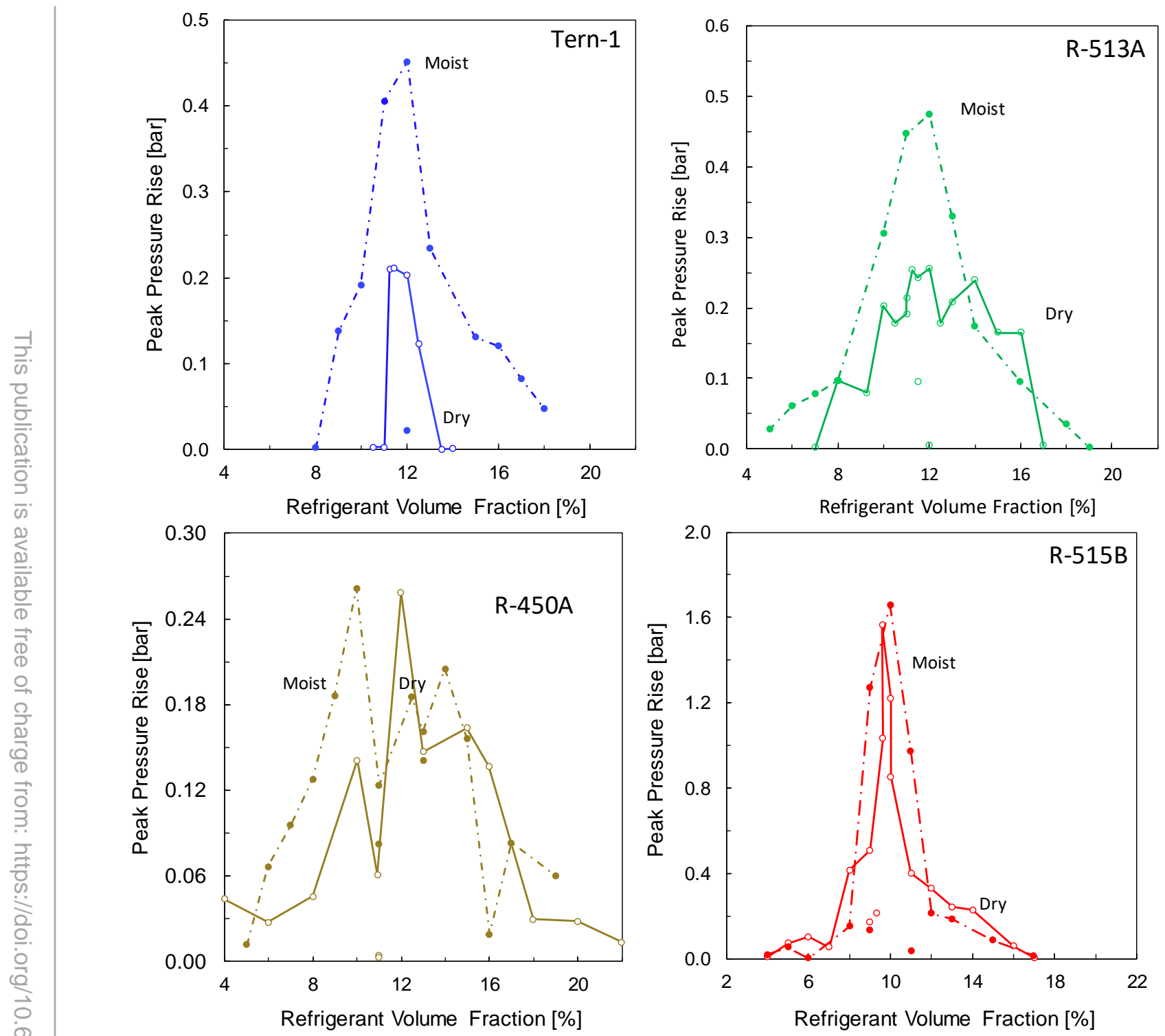

Figure 3.2-4. Explosion pressure of candidate blends (Tern-1, R-513A, R-450A, and R-515B) in JHPGL test as a function of blend volume fraction in air (solid lines: dry air; dashed lines: moist air; note different scales). 

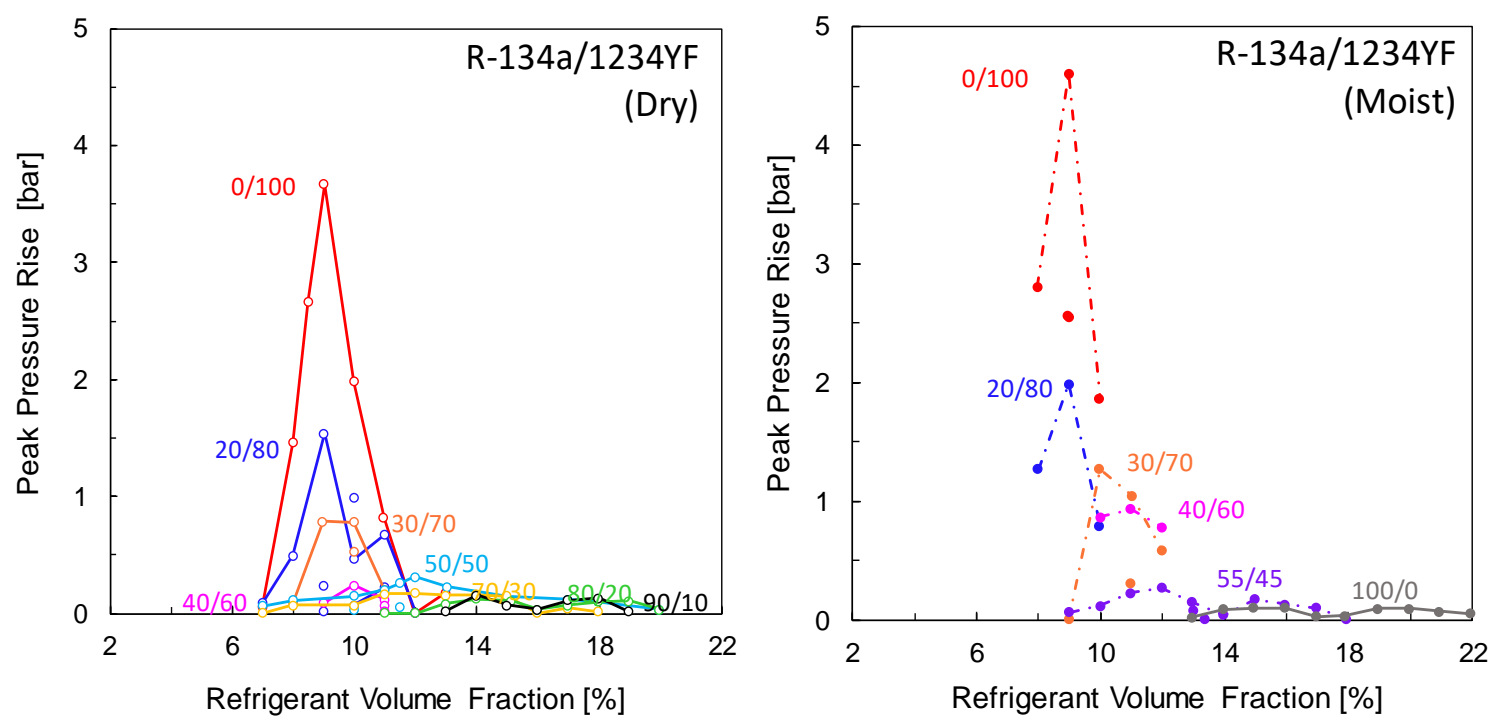

Figure 3.2-5. Explosion pressure in the JHPGL test for binary blends of HFC-134a with HFO-1234yf as function of volume fraction the blend in air. Different curves show results for varying volume fractions of HFC-134a/HFO-1234yf in the blend (left frame: dry air; right: moist).

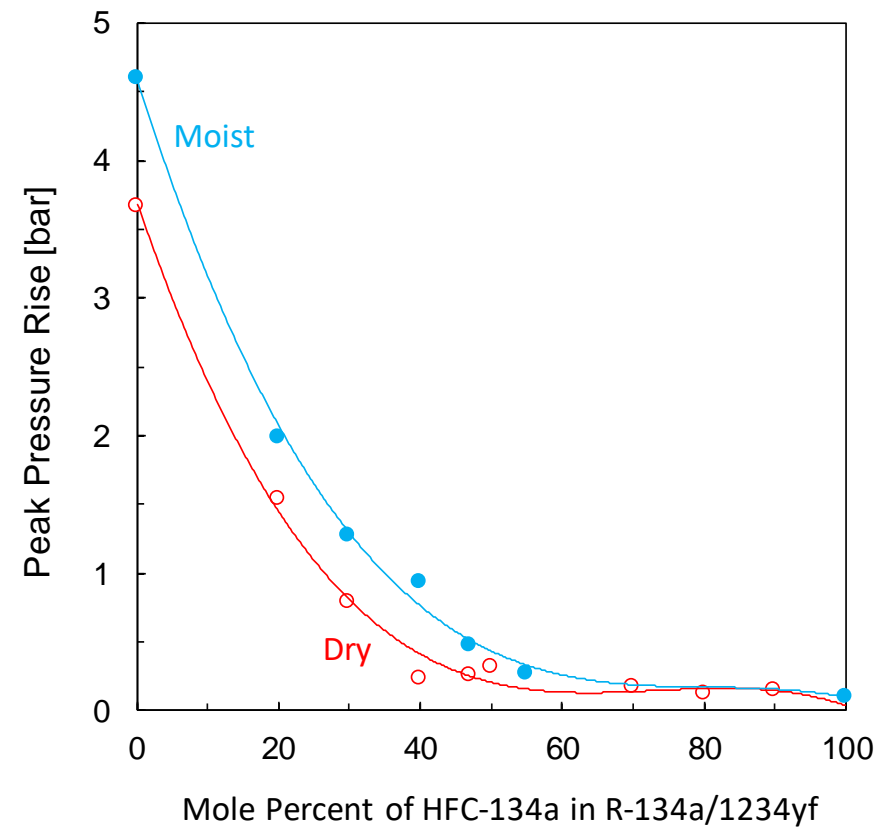

Figure 3.2-6. Peak explosion pressure in the JHPGL test for binary blends of HFC-134a with HFO-1234yf as function the volume fraction of HFC-134a in the blend, for moist (50\% r.h.) and dry (<2\% r.h.) air. 


\subsubsection{Improvements in Predictive Model}

Recent work at NIST has had the goal of developing the predictive capability for flammability. A reasonable surrogate for flammability is the laminar burning velocity $S_{\mathrm{u}}{ }^{0}$, which is defined as the rate of propagation of a 1-D, planar, adiabatic, combustion wave through a mixture of combustible gases. It is a useful flammability metric since it is a fundamentally based parameter incorporating the effects of overall reaction rate, heat release, and molecular transport for the fuel-oxidizer mixture. It is used as a scaling parameter for turbulent flame speed ${ }^{32}$ and as an input to full-scale explosion models ${ }^{33,34}$. It is correlated with minimum ignition energy, flame quenching diameter, and lean flame extinction, and is a metric used in existing and developing codes and standards for refrigerant flammability ${ }^{17,18}$. In the present work, we adopt burning velocity, along with the overall chemical rate as determined in a stirred-reactor, since both have proven useful for understanding the behavior of near-limit flames of fluorinated compounds, alone with air, or in hydrocarbon-air mixtures $15,35-43$. Calculating either the burning velocity or the overall chemical rate requires a detailed kinetic model of the combustion of the fluorinated compounds.

Early work at NIST developed a detailed kinetic model for HFC fire suppressants ${ }^{44,45}$ and recent work has extended it to flames of pure refrigerants and air ${ }^{46-48}$. In the present project, the mechanism was further refined and updated with new rates and thermodynamic data appearing in the literature. As a result, the mechanism can accurately predict the existing experimental burning velocity data in the literature for one- and two-carbon HFCs ${ }^{47}$ and fluoropropenes (HFOs) ${ }^{48}$. Since it has not been extensively tested with data for blends of refrigerants, the present work collected new burning velocity data for R-152a/134a, and R-152a/1234yf blends. Also, since the burning velocity of either HFO-1234yf or HFC-134a with air at $298 \mathrm{~K}$ is too low to measure in normal-gravity experiments, the addition of a more reactive HFC, in this case HFC-152a, allows one to more readily obtain data on their combustion behavior. The mechanism was then used to calculate the burning velocity $a$ priori for the experimental conditions to assess its predictive ability.

Experimental Data for Model Validation. As described in Section A.2.3 of the Appendix, the $2 \mathrm{~L}$ chamber was used with a spark ignition system to collect the chamber pressure $P$ as a function of time $t$, from which the burning velocity is determined using a thermodynamic model ${ }^{49}$. Figure 3.2-7 shows the burning velocity as a function of refrigerant volume fraction ${ }^{50}$ (points: experimental data, dotted lines: cubic fit to experimental data, solid lines: numerical predictions). The different curves in each frame are for R-152a/134a blends at (1.00/0.0), (0.80/0.20), (0.50/0.50) volume fractions (left frame), and R-152a/1234yf blends at $(1.00 / 0.0),(0.50 / 0.50),(0.40 / 0.60)$, and $(0.30 / 0.70)$ volume fraction. The air in these tests was dry $\left(<2 \%\right.$ r.h. at $\left.23{ }^{\circ} \mathrm{C}\right)$ except for the one curve for at R-152a/1234yf $(0.40 / 0.60)$, for which the r.h. was about $50 \%$. The dotted lines are cubic fits to the experimental data. As indicated, the mechanism does a very good job of predicting the burning velocity for these conditions. A similar comparison has been performed for other refrigerants ${ }^{46-48}$. The different data sets on each frame correspond to different molar ratios of the two constituents, as indicated on the curve labels. The experimental data are the points, the dotted lines cubic fits to the experimental data, and the solid lines the numerical modeling predictions. 

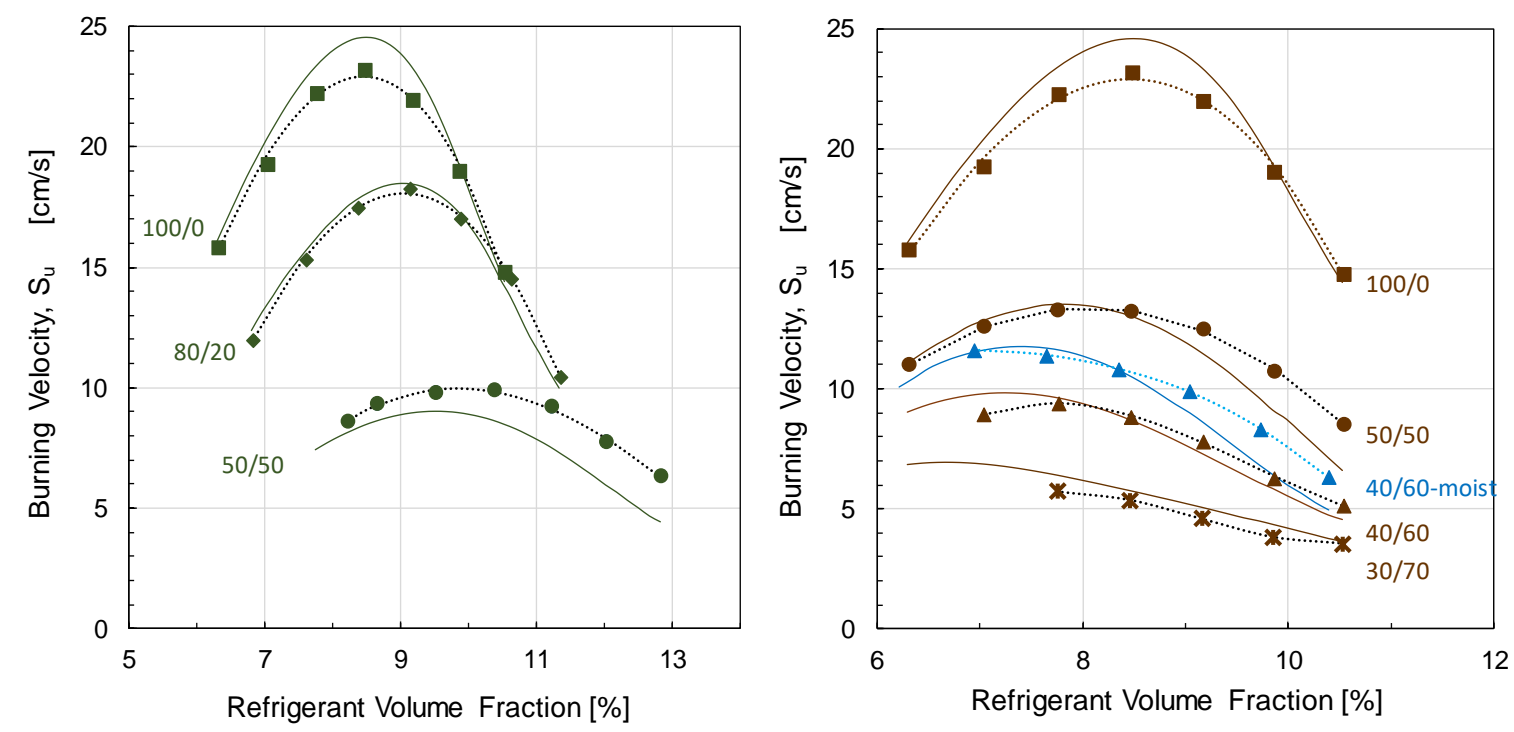

Figure 3.2-7. Laminar burning velocity of R-152a/134a (left frame) and R-152a/1234yf (right frame) mixtures with air as a function of the equivalence ratio.

Figure 3.2-8. summaries the predictions of the present model vs. the experimental results, indicating that the predictive ability of the model is good. Hence, the kinetic model should be a useful tool for understanding the present candidate blends (since all of the constituents of the blends are included in the kinetic model).

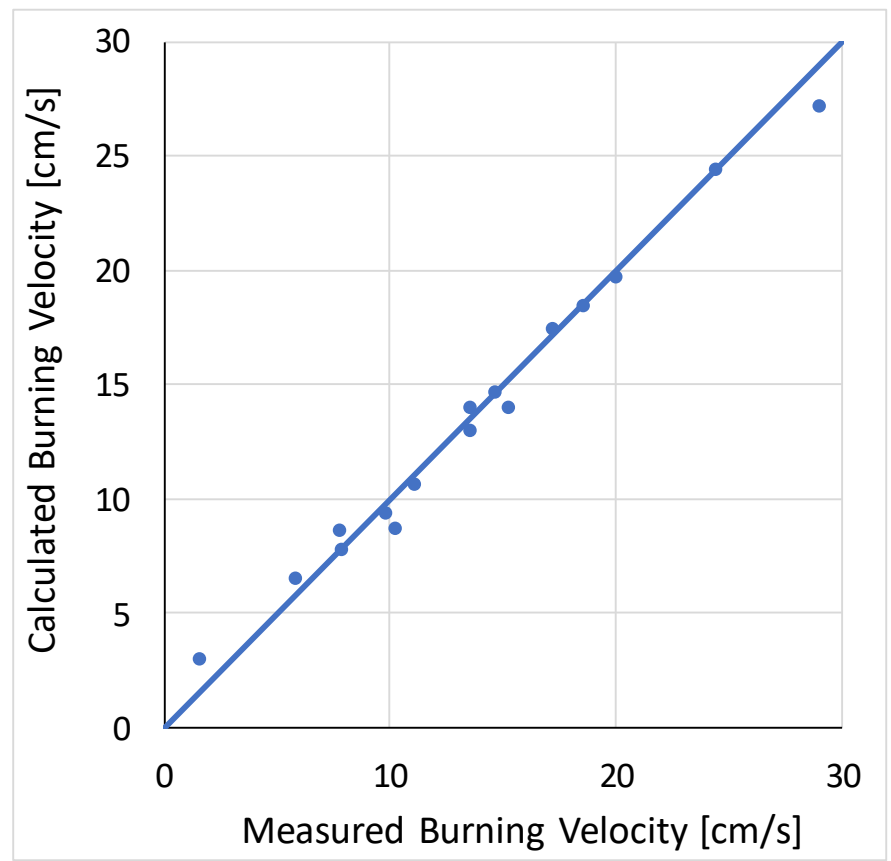

Figure 3.2-8. Calculated vs. measured laminar burning velocity (peak over all $\phi$ ) of twentyone refrigerant $/ \mathrm{N}_{2} / \mathrm{O}_{2}$ flames for which experimental data are available in the literature ${ }^{46-48}$. 


\subsubsection{Interpretation of E681 and JHPGL Experimental Results}

The results in the JHPGL test as well as in the E681 test can be interpreted using the kinetic model as a tool. A useful metric is the overall reaction rate of the mixtures, at the conditions of interest (e.g., initial $T, P$, humidity, etc.). Simulations (or experiments) of the blow-out condition of a perfectly stirred reactor (PSR) can be used to obtain an estimate of the overall chemical rate $\omega_{\text {psr. }}$ This rate can be used, among other things, to estimate the laminar burning velocity ${ }^{51}$, the flammability limits ${ }^{16}$, and the extinction conditions of full-scale fires ${ }^{39}$. For example, in Figure 3.2-9, the overall chemical rate $\omega_{\mathrm{psr}}$ calculated for the mixture $\mathrm{R}-1234 \mathrm{yf} / 134 \mathrm{a}$ is shown as a function of the volume fraction of the blend (i.e., fuel). Increasing volume fractions of HFC-134a in the blend reduces $\omega_{\mathrm{psr}}$, and the effect of humidity is shown by the three frames $(0 \%$ r.h., $50 \%$ r.h., and $100 \%$ r.h.). Of particular interest is the peak value of $\omega_{\mathrm{psr}}$ for each blend. These are shown in Figure 3.2-10 with one curve for each value of the humidity in the air. As indicated, increasing water vapor $(0 \%$ to $100 \%$ r.h.) increases the peak overall reactivity by a factor of 2.5 for HFO-1234yf and 1.8 for HFC-134a. Moreover, as shown in Figure 3.2-9, at $50 \%$ r.h. the $\omega_{\text {psr }}$ has broad plateaus, whereas at 0 and $100 \%$ r.h. there are distinctive peaks. This behavior is expected to be exhibited for all HFC blends.
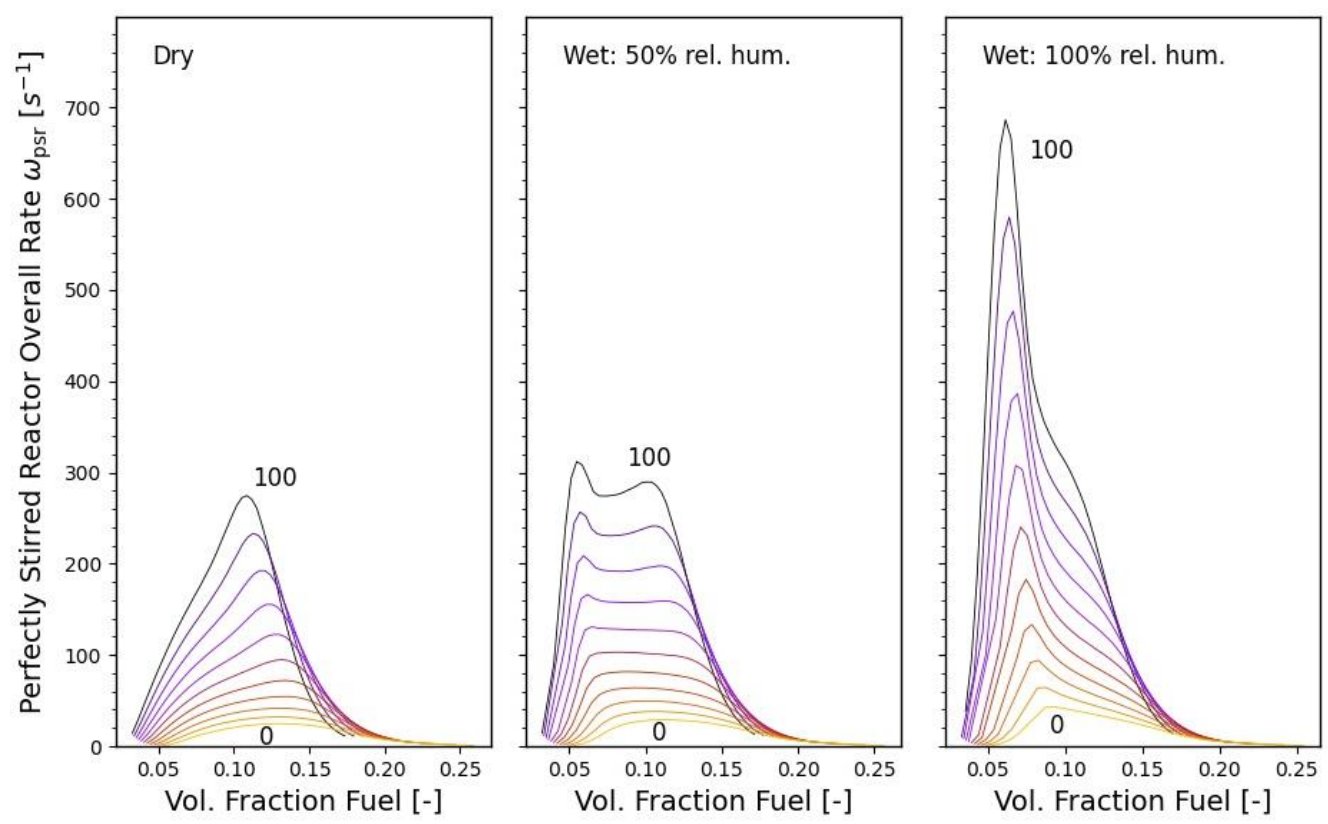

Figure 3.2-9. Overall chemical reaction $\omega_{\mathrm{psr}}$ as a function of volume fraction in air determined via perfectly stirred reactor simulations for the blend R1234yf/134a. Each curve refers to one blend ratio: top curve, 100\% HFO1234yf, bottom curve $100 \%$ HFC-134a; Frames: $0 \%$ r.h. (dry), $50 \%$ r.h., and $100 \%$ r.h. 


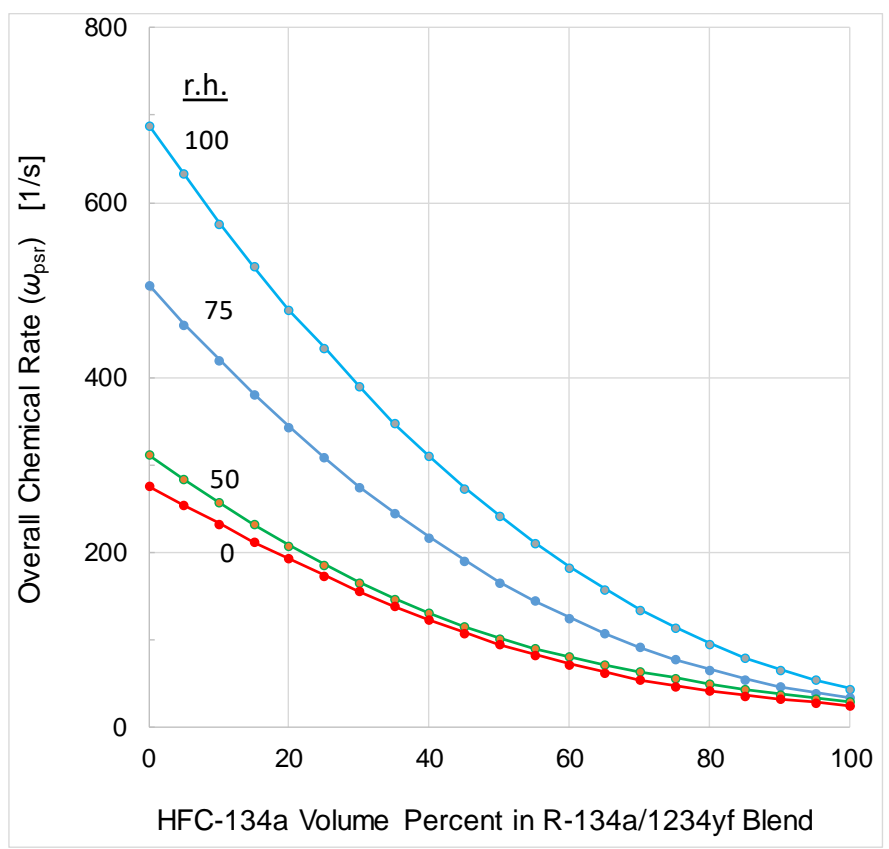

Figure 3.2-10. Peak (over all $\phi$ for each blend) chemical reaction $\omega_{\mathrm{psr}}$ as a function of volume fraction of HFC-134a in the blend of R-1234yf/134a. Individual curves are shown for varying water vapor in the air $(0 \%, 50 \%, 75 \%$ and $100 \%$ r.h.)

The overall chemical rate $\omega_{\text {psr }}$ provides a useful metric for examining the flammability of refrigerant blends. It can be used to compare alternative flame-suppressant agents, examine their flammability with respect to concentration in the air, discern the effects of humidity, and compare with experimental results. It is used in the section below to interpret the experimental results in the E681 and JHPGL tests.

Figure 3.2-11 shows the explosion pressure in the JHPGL test vs. the Overall Chemical Rate $\omega_{\text {psr }}$ for data from the candidate blends (" $X$ " markers) and the R-1234yf/134a blends (" $O$ " markers) (from Table 3.2-1). Blue points have an ASHRAE Standard 34 Class 1 rating, and the blue box bounds the locus of those mixtures; the orange points are class $2 \mathrm{~L}$. As indicated, there is a good correlation between the explosion pressure and $\omega_{\mathrm{psr}}$. As described above, the three blends Tern-1, R-513A, and R-450A have about the same explosion pressure and $\omega_{\mathrm{psr}}$. The blend R-515B, while it has a Class 1 rating, is likely at the limit of this rating because the 70/30 R1234yf/134a blend is just at the Class 1 limit (i.e., 72/28 and 74/26 had flame angles $>90^{\circ}$ and hence were not Class 1). Thus, the three metrics, ASHRAE Standard 34 rating, JHPGL test explosion pressure, and $\omega_{\text {psr }}$ are related as shown in Figure 3.2-11. For a Class 1 rating, the explosion must be less than 1.41 bar \pm 0.14 bar and $\omega_{\mathrm{psr}}<166 \mathrm{~s}^{-1}$. The important point is that $\omega_{\text {psr }}$ is a parameter based in physics that can be calculated for a mixture and can be used to correlate experimental flammability results between test methods, or with full-scale test results.

Interpretation of Results with Regard to the Full-Scale Live-Fire Tests. As shown in Figure 3.2-11, the JHPGL test explosion pressure and $\omega_{\mathrm{psr}}$ are useful scales for characterizing blends. The next step is to understand, for the live-fire tests, what degree of flammability is 
acceptable. A straightforward way to do that would be do live-fire tests with mixtures of $\mathrm{R}-1234 \mathrm{yf} / 134 \mathrm{a}$ at varying mole fractions of HFC-134a. The mixture ratio that provides nonflammable behavior in that test can be used with Figure 3.2-11 to quantify the corresponding JHPGL test explosion pressure limit and $\omega_{\text {psr }}$ limit.

The overall chemical rate $\omega_{\text {psr }}$ can also be used to explore other features of the blends. For example, Figure 3.2-12 shows $\omega_{\text {psr }}$ for the candidate blends for different levels of water vapor in the air $(0 \%, 50 \%$, and $100 \%$ r.h.) Data are shown for the nominal blend (Nom), worstcase fractionation WCF (from uncertainty in the blend components), and worst-case fractionation for flammability WCFF (from different vaporization rates for leaking blend components of liquid agent at the WCF). As indicated, for most blends, the differences in Nom., WCF, and WCFF are small, except for the case of R-450A, for which the WCFF is quite different from the WCF (although the Class 1 rating is still maintained for the WCFF). In the DOD application, the concern is with rapid loss of refrigerant charge, so WCF is the mixture of interest. A humidity level of $50 \%$ r.h. was selected for the JHPGL tests as listed in the comparisons in Table 3.2-1. Nonetheless, as discussed for the R-1324yf/134a blend in Figure 3.2-10, Figure 3.2-12 shows that for the candidate blends, although there is predicted to be a small effect of humidity at $50 \%$ r.h., there is predicted to be a large effect of humidity at $100 \%$ r.h.

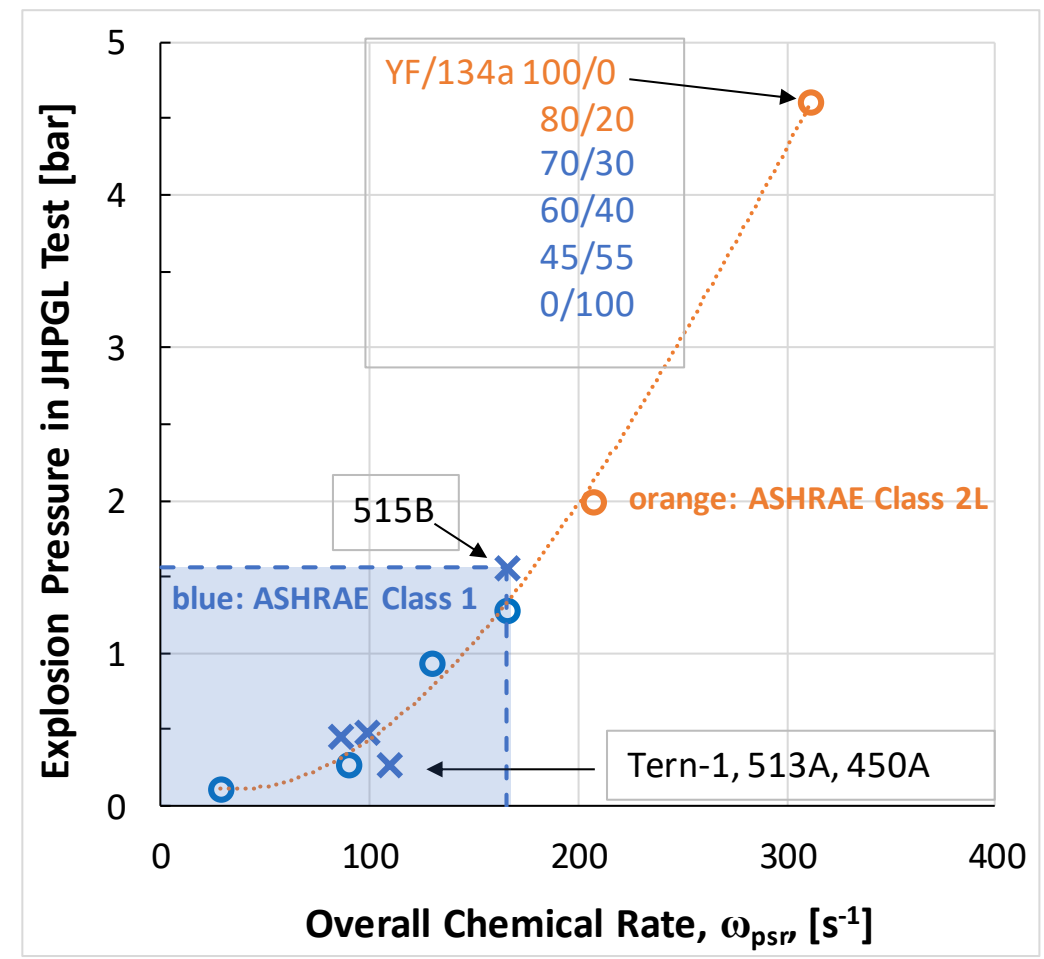

Figure 3.2-11. Explosion pressure in the JHPGL test vs. overall chemical reaction $\omega_{\text {psr }}$ (both peak over all $\phi$ ) for R-513A, R-450A, R-515B and the Tern-1 blend and the $\mathrm{R}-1234 \mathrm{y} / 134 \mathrm{a}$ blends at increasing HFC-134a volume fraction. Dotted line is a polynomial fit to all the data to aid in visualization. (Initial conditions: $296 \mathrm{~K}, 101 \mathrm{kPa}, 50 \%$ r.h.) 

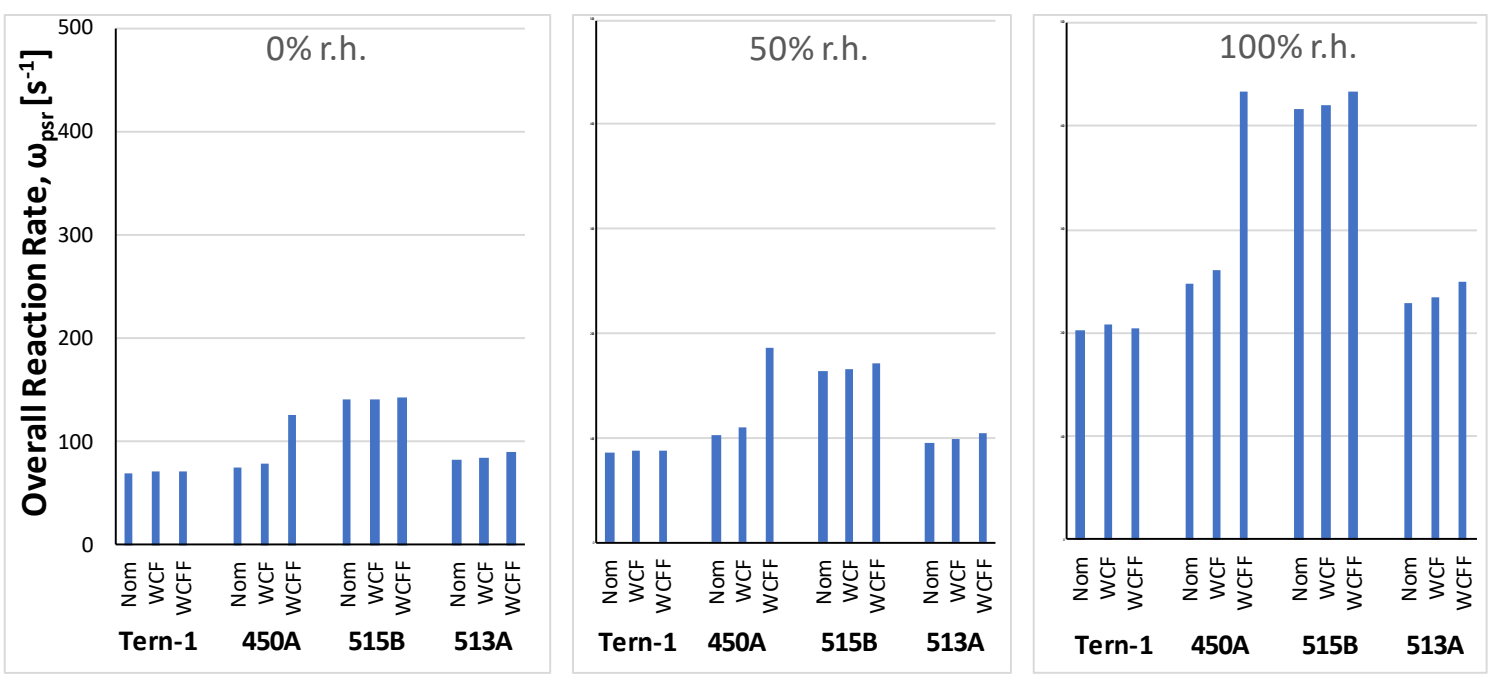

Figure 3.2-12. Peak (over all $\phi$ for each blend) chemical reaction $\omega_{\mathrm{psr}}$ for R-513A, R-450A, R-515B and the NIST blend. Data are shown for the Nominal, WCF, and WCFF compositions in air $(T=296 \mathrm{~K})$ and $0 \%, 50 \%$, and $100 \%$ r.h.

\subsubsection{Coordination with Full-Scale Testing}

The live-fire tests at ARL have been delayed due to COVID-19 related shutdowns. NIST will work with the engineers at ARL to provide input on potentially useful tests to conduct, as well as provide interpretation using the computational tools that have been developed. For example, based on the results to date:

1. The three blends, Tern-1, R-513A, and R-450A are expected to behave similarly and have the most promising behavior with respect to flammability. R-515B, while nonflammable in the E681 test, it is close to the border of flammability.

2. Levels of humidity above 0.014 moles $\mathrm{H}_{2} \mathrm{O} /$ mole air $\left(50 \%\right.$ r.h. at $\left.23{ }^{\circ} \mathrm{C}\right)$ may significantly increase the flammability of the blends.

3. HFC-134a should be tested at high ambient temperature and high humidity as a benchmark. Following that, tests with increasing amounts of added HFO-1234yf would be very valuable.

Recommendations concerning a proposed test matrix with ARL engineers will be discussed. The results of the present tests and calculations will be used as input. The goal is to minimize the number of full-scales tests while extracting as much information as possible from the tests. It is possible that the ASHRAE Standard 34 Class 1 flammability rating will be sufficient to ensure non-flammability in the live-fire tests. It is also possible that a more stringent criterion, for example something close to the behavior of Tern-1, R-513A and R450A in Figure 3.2-11 $\left(P_{\max }<0.3\right.$ bar and $\left.\omega_{\mathrm{psr}}<110 \mathrm{~s}^{-1}\right)$ will be required. With a few livefire tests, it should be possible to bound and understand the problem, and additional tests would provide higher levels of confidence in the findings and conclusions. 


\subsubsection{Summary of Flammability Work to Date}

All of the four candidate blends are non-flame propagating in the modified E681 test specified in ASHRAE Standard 34. In a more stringent test, the Japanese high-pressure gas law test (JHPGL), the explosion pressure in a $2 \mathrm{~L}$ combustion chamber with a fused platinum wire ignition source, is used as a metric for flammability. Three of four candidate blends (Tern-1, R-513A, R-450A) had similar pressure rise (0.451, 0.474, and 0.262 bar), while one (R-515B) had a higher pressure rise (1.56 bar). Tests with binary blends of HFC-134a and HFO-1234yf with increasing fraction of HFC-134a showed that an HFC-134a mole fraction of 0.30 was required to pass the E681 tests, and at this composition, the explosion pressure in the JHPGL test was 1.27 bar. Hence, it appears that the R-515B blend is close the edge of passing the E681 test, while the other blends pass the test more easily.

A recently developed and validated kinetic model of combustion for one-, two-, and threecarbon HFC and HFO refrigerants was further improved and validated. For this purpose, burning velocity data were obtained in the $2 \mathrm{~L}$ chamber from the rate of pressure rise (using a spark ignition system). For binary blends of R-152a/134a and R-152a/1234yf, the experimentally derived burning velocity agreed well with the predictions from detailed numerical simulations. This adds confidence to the kinetic model.

The kinetic model was subsequently used to estimate the overall chemical reaction rate of the candidate blends. Both the E681 flame propagating/non-propagating boundary, as well as the JHPGL test explosion pressure were well correlated with the calculated overall reaction rate for each blend. The overall reaction rate is easily calculated for any arbitrary mixtures of interest. It is a fundamentally based parameter that can be used to correlate experimental flammability results between test methods, or with full-scale test results.

An important question is how well the live-fire tests at the Army Research Laboratory will compare with the small-scale E681, the JHPGL tests, and the calculated overall rate. It is unclear a priori if the live-fire tests will require lower or higher levels of flammability than the E681. For example, turbulence can increase flame speed of premixed flames, but high velocities can strain flames and extinguish them. If live-fire tests can be conducted for the mixtures of HFC-134a/HFO-1234yf at increasing mole fraction of HFC-134a until acceptable flammability occurs, it will presumably be possible to correlate the behavior in the three metrics (E681, JHPGL test, and calculated overall reaction rate), greatly reducing the number of required tests and increasing the information available from each test. 


\subsection{Task 3: Testing of Selected Blends in a Mini-Breadboard Heat Pump}

The Mini-Breadboard Heat Pump (MBHP) was used to experimentally evaluate HFC-134a and four candidate low-GWP blends: R-513A, R-450A, R-515B, and Tern-1. Section 3.5.2 details how these blends were selected. The purpose of these tests was to validate the CYCLE_D-HX simulation model ${ }^{52,53}$ used in the limited-scope project ${ }^{1}$ and qualify the three 'best' blends for testing in a military ECU (Task 7).

Note the MBHP measurements were de facto 'drop-in tests' and do not show the absolute performance potential for each fluid, since the MBHP hardware was not optimized for each fluid. The limited-scope project simulations included hardware optimized for each fluid, and thus provide a more equitable fluid comparison.

\subsubsection{Test Apparatus}

The NIST MBHP is a laboratory, modular heat-pump system for measuring performance of refrigerants. This system is extensively instrumented. The refrigerant circuit includes a variable-speed, oil-lubricated, reciprocating compressor powered by an electric motor and inverter, where the speed controls cooling/heating capacity. The evaporator and condenser are single circuits (no parallel tube branches). The heat exchangers' size can be adjusted by changing the number of active refrigerant tubes, which enables control of the heat flux. The evaporator and condenser are of the annular design arranged in the counter-current configuration; the refrigerant flows in the enhanced inner tube (copper), while the heattransfer fluid (HTF) flows in the smooth annular space. An electronic expansion valve (EEV) regulates the evaporator-exit superheat. A liquid-line/suction-line heat exchanger (LLSLHX) can be optionally included in the refrigerant circuit. The small internal volume of this apparatus facilitates testing fluids that are only available in small quantities. A chiller removes heat from the condenser, and a water heater applies a load to the evaporator. More details about the test apparatus are available in ${ }^{55}$.
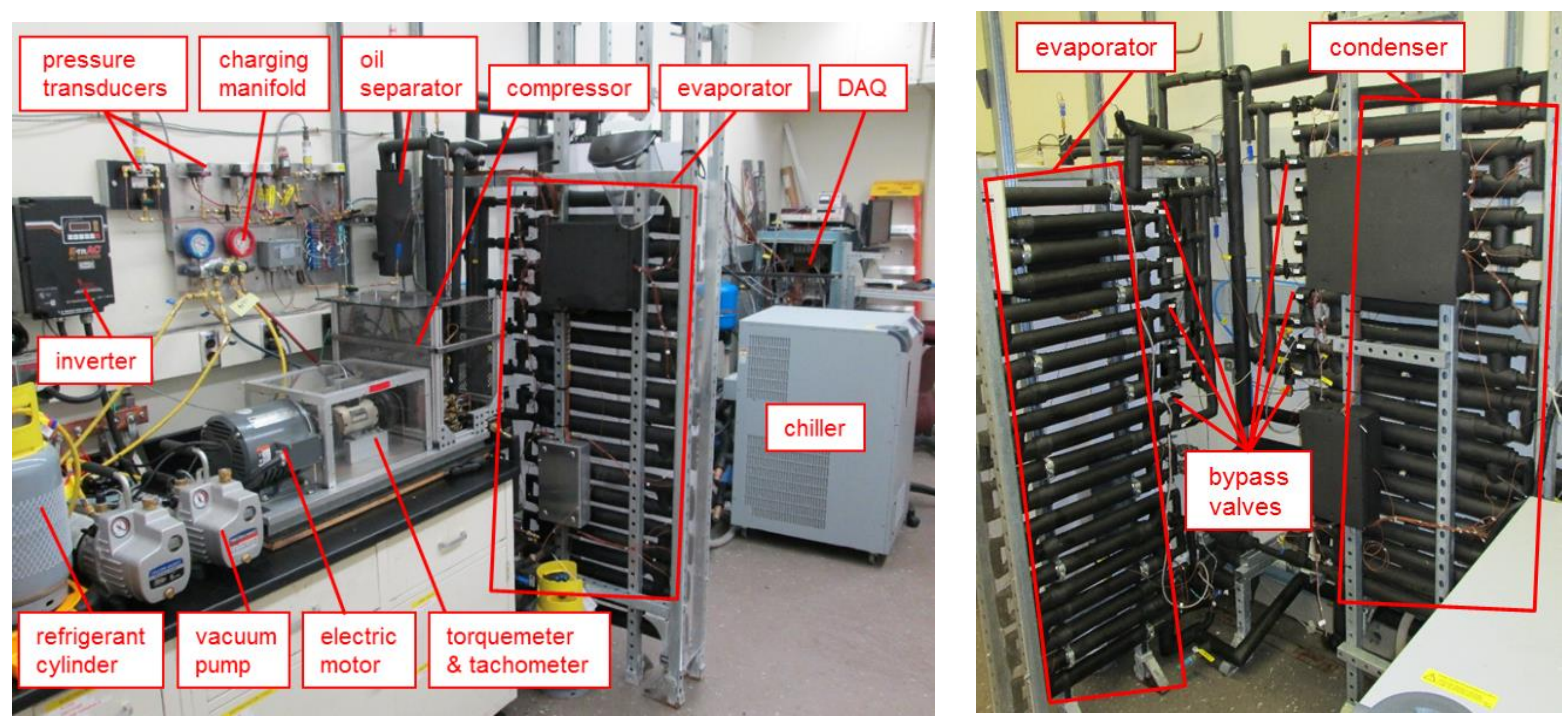

Figure 3.3-1. Photos of the MBHP test apparatus. 


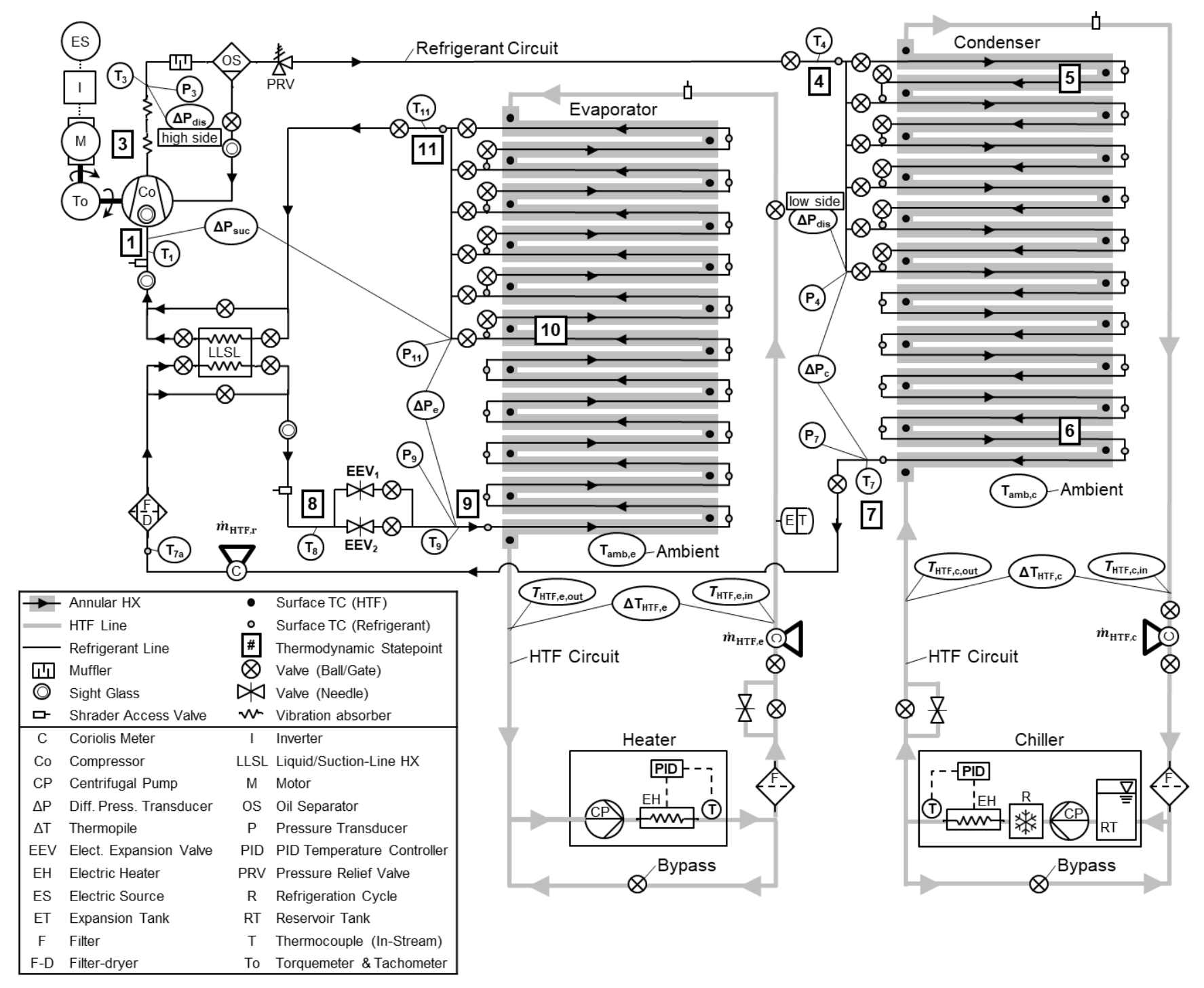

Figure 3.3-2. Schematic of the MBHP test apparatus. 


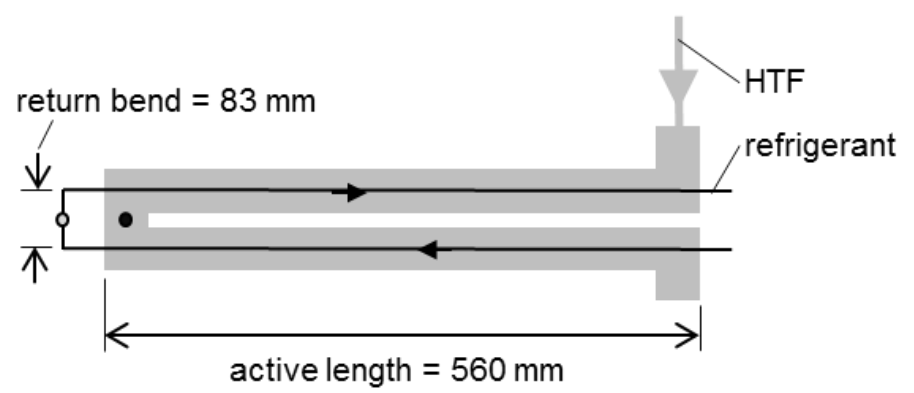

(a)

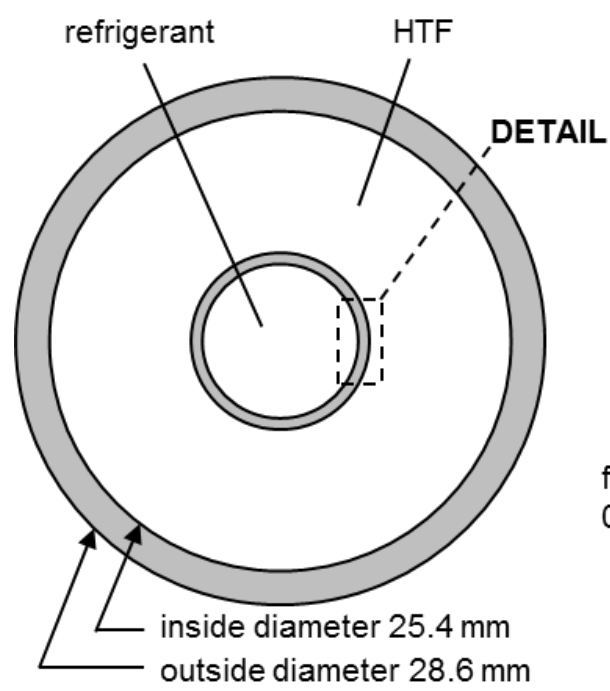

(b)

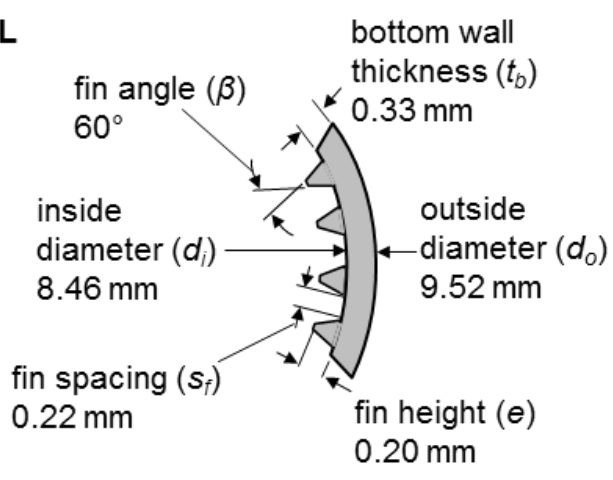

DETAIL

(c)

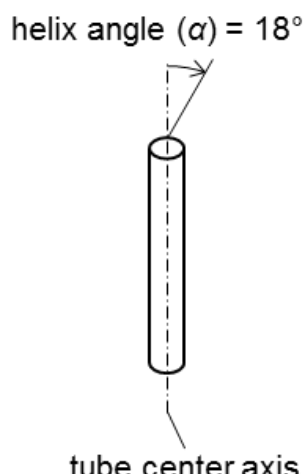

(d)

Figure 3.3-3. MBHP: Schematics of annular heat exchanger including (a) refrigerant tube lengths, (b) cross section of annular heat exchanger, (c) detailed cross-section of microfin tube, and (d) helix angle of microfins.

\subsubsection{Test Protocol}

The first baseline tests with HFC-134a established the control parameter settings required to reach the operating targets listed in Table 3.3-1. The HTF inlet temperatures were set to achieve refrigerant average saturation temperatures of $40{ }^{\circ} \mathrm{C}$ in the condenser and $8{ }^{\circ} \mathrm{C}$ in the evaporator, which are typical for air-source heat pumps operating at the Cooling A rating test ${ }^{54}$. The compressor speed was controlled to generate a cooling capacity of $1.5 \mathrm{~kW}$. Condenser and evaporator dewpoint temperature drops of $2 \mathrm{~K}$ were targeted since the CYCLE_D-HX model showed the HFC-134a mass flux was optimal under these conditions (the mass flux was high enough for good heat transfer but with only moderate pressure drop penalty). These dewpoint temperature drops were also used in the limited-scope project ${ }^{1}$. These dewpoint temperature changes were obtained using 10 evaporator tubes and 16 condenser tubes. A refrigerant charge of $1420 \mathrm{~g}$ produced the targeted subcooling of $5 \mathrm{~K}$. Extensive tests showed that an evaporator-exit superheat of $15 \mathrm{~K}$ yielded the best repeatability for the system without the LLSL-HX. A lower evaporator-exit superheat of $8 \mathrm{~K}$ was used with the LLSL-HX to achieve nominally equivalent compressor suction superheat (which correlated to repeatability), and because the lower superheat resulted in lower LLSL-HX energy 
imbalance. The condenser HTF was distilled water and the evaporator HTF was a potassium formate brine (Dynalene HC40) whose capacitance was measured and reported in ${ }^{55}$.

After establishing the baseline conditions and heat exchanger sizes, HFC-134a and the four replacement candidates were tested at varied capacity (Table 3.3-2, Table 3.3-3) to quantify performance over a range of heat and mass fluxes, for a total of 121 tests. Test conditions were repeated to quantify representative average performance and to bring the $95 \%$ confidence for COP to the (0.5 to 1.0$) \%$ range, where the variation was largely driven by test-to-test differences in compressor efficiency (more details given in Section 3.3.4). The tests were primarily carried out at $(1.3,1.5$ and 1.7$) \mathrm{kW}$ capacity. Additional tests at higher capacities of (1.9 and 2.0) kW were also performed for HFC-134a, R-513A, and R-450A, but these two test points were abandoned for Tern- 1 and R-515B because it became clear the refrigerant mass flux and pressure drop too high to represent realistic conditions for a system with optimization of evaporator and condenser refrigerant tube circuitry.

The condenser and evaporator HTF inlet temperatures and number of tubes were fixed for all tests at the values established in the baseline tests. For every test the compressor speed was set to reach the target capacity, and the refrigerant charge was adjusted to achieve the target subcooling. All fluids were tested with and without the LLSL-HX. Energy imbalance between the refrigerant and HTF in the condenser and evaporator was less than $5 \%$.

Table 3.3-1. MBHP: HFC-134a baseline test operating targets and control parameters.

\begin{tabular}{llc|lc}
\hline$\#$ & Operating parameter target & Value & Control Parameter & Value \\
\hline 1 & Avg. saturation temp - cond. & $40^{\circ} \mathrm{C}$ & HTF inlet temp: cond. & $32.7^{\circ} \mathrm{C}$ \\
2 & Avg. saturation temp - evap. & $8{ }^{\circ} \mathrm{C}$ & HTF inlet temp: evap. & $28.0{ }^{\circ} \mathrm{C}$ \\
$3 \mathrm{a}$ & Cooling capacity (w/o LLSL-HX) & $1.5 \mathrm{~kW}$ & Compressor speed (w/o LLSL-HX) & $14.5 \mathrm{~Hz}$ \\
$3 \mathrm{~b}$ & Cooling capacity (w/ LLSL-HX) & $1.5 \mathrm{~kW}$ & Compressor speed (w/ LLSL-HX) & $13.5 \mathrm{~Hz}$ \\
4 & Dewpoint temp. drop - cond. & $2 \mathrm{~K}$ & Number of tubes: cond. & 16 \\
5 & Dewpoint temp. drop - evap. & $2 \mathrm{~K}$ & Number of tubes: evap. & 10 \\
6 & Subcooling & $5 \mathrm{~K}$ & Refrigerant charge & $1420 \mathrm{~g}$ \\
$7 \mathrm{a}$ & Superheat - w/o LLSL-HX & $15 \mathrm{~K}$ & EEV opening & -- \\
$7 \mathrm{~b}$ & Superheat - w/ LLSL-HX & $8 \mathrm{~K}$ & EEV opening & -- \\
\hline
\end{tabular}


Table 3.3-2. MBHP: Test targets and parameters.

\begin{tabular}{|c|c|c|c|c|c|c|c|}
\hline Parameter & Unit & Tol. & & & Value & & \\
\hline Cooling capacity & $\mathrm{kW}$ & $\pm 2 \%$ & 1.3 & 1.5 & 1.7 & 1.9 & 2.0 \\
\hline HTF temperature change: evap. & K & \pm 0.02 & 8.67 & 10.00 & 11.33 & 12.67 & 13.33 \\
\hline HTF inlet temp: cond. & ${ }^{\circ} \mathrm{C}$ & \pm 0.2 & & & 32.7 & & \\
\hline HTF inlet temp: evap. & ${ }^{\circ} \mathrm{C}$ & \pm 0.3 & & & 27.9 & & \\
\hline HTF flowrate: cond. & $\mathrm{g} / \mathrm{s}$ & \pm 0.3 & & & 97.6 & & \\
\hline HTF flowrate: evap. & $\mathrm{g} / \mathrm{s}$ & \pm 0.3 & & & 56.3 & & \\
\hline Number of tube circuits: cond. & -- & -- & & & 1 & & \\
\hline Number of tube circuits: evap. & -- & -- & & & 1 & & \\
\hline Number of tubes: cond. & -- & -- & & & 16 & & \\
\hline Number of tubes: evap. & -- & -- & & & 10 & & \\
\hline Subcooling: cond. out & K & \pm 0.5 & & & 5 & & \\
\hline Superheat: evap. out (w/ LLSL-HX) & K & \pm 1.0 & & & 15 & & \\
\hline Superheat: evap. out (w/o LLSL-HX) & $\mathrm{K}$ & \pm 1.0 & & & 8 & & \\
\hline Energy imbalance: cond. & $\%$ & \pm 5 & & & 0 & & \\
\hline Energy imbalance: evap. & $\%$ & \pm 5 & & & 0 & & \\
\hline
\end{tabular}

Table 3.3-3. MBHP: Executed test matrix.

\begin{tabular}{llllll}
\hline \multicolumn{6}{c}{ Cooling capacity $[\mathrm{kW}]$} \\
\hline 1.3 & 1.5 & 1.7 & 1.9 & 2.0
\end{tabular}

\begin{tabular}{lllllc} 
Fluid & \multicolumn{5}{c}{ Number of tests: without (with) LLSL-HX } \\
\hline HFC-134a & $4(4)$ & $4(4)$ & $4(4)$ & $4(4)$ & $3(2)$ \\
R-513A & $3(2)$ & $3(2)$ & $3(2)$ & $3(2)$ & -- \\
R-450A & $4(3)$ & $4(3)$ & $4(3)$ & $1(0)$ & -- \\
Tern-1 & $4(3)$ & $4(3)$ & $4(3)$ & -- & -- \\
R-515B & $4(3)$ & $4(3)$ & $4(3)$ & -- & -- \\
\hline
\end{tabular}

\subsubsection{Model simulation of tests}

CYCLE_D-HX is a semi-theoretical model that simulates performance of a vaporcompression cycle for specified temperature profiles of the heat source and heat sink. The evaporator and condenser refrigerant saturation conditions (e.g. temperature, pressure) can optionally be predicted based on physical models of the two-phase heat transfer and pressure drop. To utilize this feature, CYCLE_D-HX requires, as a preliminary step, simulating of a 'reference case' to calculate the thermal resistance on the HTF side and to establish 
correlations for refrigerant heat transfer and pressure drop in the heat exchangers ${ }^{52,53}$. The inputs for the 'reference case' simulation are based on measured data for the reference fluid and also include the geometry parameters characterizing the evaporator and condenser: refrigerant tube inner diameter and length, number of tubes, number of circuits, and indication of whether the tube is smooth or enhanced (Figure 3.3-3). Further, the model can leverage this feature to optimize refrigerant tube circuitry for each fluid, enabling the equitable comparison of fluid performance potential shown in the limited-scope project ${ }^{1}$.

Each experimental test was simulated using CYCLE_D-HX to verify the model's predictive capability. Model inputs included measurements for: HTF inlet and outlet temperatures, refrigerant superheat and subcooling, compressor volumetric and isentropic efficiencies, pressure drops in the suction and discharge lines, and the target cooling capacity. The model then predicted the cycle thermodynamic states and the resulting COP and volumetric capacity $\left(Q_{\mathrm{vol}}\right)$. The model's tube circuit optimization feature was not used for simulation of the experimental tests since the tests had fixed evaporator and condenser tube circuitry (Table 3.3-2).

The baseline tests listed in Table 3.3-1 were the basis for two 'reference cases', one each for the cycle with and without the LLSL-HX. The use of two 'reference cases' was necessitated by the different superheats, $15 \mathrm{~K}$ and $8 \mathrm{~K}$, respectively, used in the cycle without and with LLSL-HX, respectively. Relying on a single 'reference case' could result in inadequate performance predictions because CYCLE_D-HX, as a simplification, estimates the pressure drop and heat transfer in the superheat section based on the values calculated for the twophase section. The effect of the superheat section is partially corrected for in the 'reference' HTF-side thermal resistance and refrigerant-side pressure drop multiplication factor. However, the accuracy of this correction diminishes if the superheat is different than that used to establish the 'reference', so we created two unique 'references' for tests with and without the LLSL-HX.

\subsubsection{Test Results and CYCLE_D-HX Model Validation}

It is critical to note that the MBHP measurements reported here were taken primarily for validating the CYCLE_D-HX simulation results from the limited-scope project ${ }^{1}$, rather than to provide the absolute performance potential of the candidate low-GWP fluids. While the MBHP tests were controlled to achieve a similar heat flux through the evaporator (a key requirement for a fair experiment-based comparison of different fluids ${ }^{56}$ ), all MBHP hardware was fixed for all tests, which constituted 'drop-in' testing. Such testing may not affect the results significantly for fluids having similar volumetric capacities, however, its effect on results may increase exponentially for large disparities in $Q_{\text {vol. }}$. In such a case, the system performance can be impacted to a significant degree through a performance degradation of the compressor and heat exchangers, which were optimized for the reference refrigerant. The CYCLE_D-HX validation concept relies on the assumption that if the model can correctly predict the MBHP 'drop-in' test results, it's predictions from the limited-scope project for optimized systems can be considered as verified.

Test Results. The HFC-134a replacement candidate's performances were evaluated based on cooling COP (Figure 3.3-4) and $Q_{\text {vol }}$ (Figure 3.3-5). The COP and $Q_{\text {vol }}$ data were correlated 
to capacity with a linear regression, and the figures show the individual measurements (symbols), curve fits (short-dashed lines), $95 \%$ confidence intervals of the fit (long-dashed lines). When fluid is described here as having higher tested COP (or $Q_{\mathrm{vol}}$ ) than another fluid, the curve fit value is higher, and the confidence intervals don't overlap. If the confidence intervals overlap, the average performances of the fluids have no statistical difference (NSD).

The following results summary applies to both tests without and with the LLSL-HX:

- $\quad$ COP (Figure 3.3-4)

O HFC-134a had higher COP than all replacement candidates for all capacities.

- R-515B had lower COP than all other fluids for all capacities.

○ At $1.3 \mathrm{~kW}, \mathrm{R}-513 \mathrm{~A}, \mathrm{R}-450 \mathrm{~A}$, and Tern-1 had NSD in COP.

$\circ$ At (1.5 and 1.7) kW, R-513A and Tern-1 had NSD in COP, but both had higher COP than R-450A.

- $Q_{\text {vol }}$ (Figure 3.3-5)

○ HFC-134a and R-513A had the highest $Q_{\text {vol }}$ (NSD between them), followed by Tern-1, R-450A, and R-515B.

For all tests the LLSL-HX increased COP and $Q_{\text {vol }}$ by about ( 8 to 10$) \%$.

The lower performance of R-450A and R-515B can be mostly related to their lower $Q_{\mathrm{vol}}$, $13.3 \%$ and $26.2 \%$, respectively, in relation to HFC-134a. The COP lines for R-450A and R-515B (Figure 3.3-4) reflect the trends in compressor isentropic efficiency (Figure 3.3-6). The lower $Q_{\text {vol }}$, and sometimes lower compressor volumetric efficiency (Figure 3.3-7), required higher compressor speeds for R-450A and R-515B (Figure 3.3-8). The associated increase in frictional losses caused the compressor isentropic efficiency to be low. The MBHP measurements also showed a larger pressure drop for these two blends compared to the other fluids tested, especially in the evaporator. Note these data do not constitute the absolute potential for R-450A and R-515B since the MBHP hardware was not optimized for these fluids. The approach used in the limited scope simulation study ${ }^{1}$ gave a more fair comparison of fluid performance potential, since all fluids were evaluated with the same compressor isentropic efficiency and the heat exchanger circuitry was optimized for each fluid. 


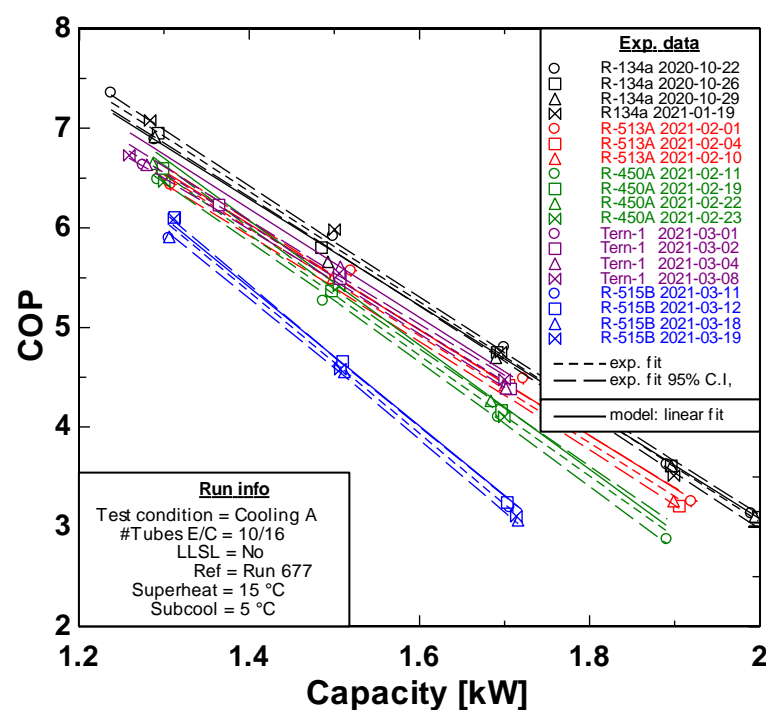

(a)

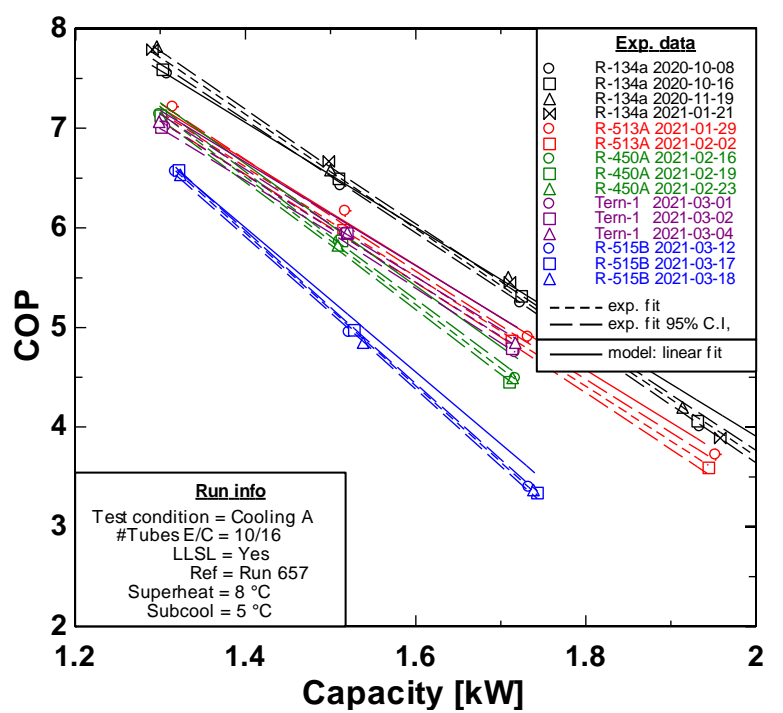

(b)

Figure 3.3-4. Cooling COP for HFC-134a and the replacement candidates (a) without LLSLHX and (b) with LLSL-HX.

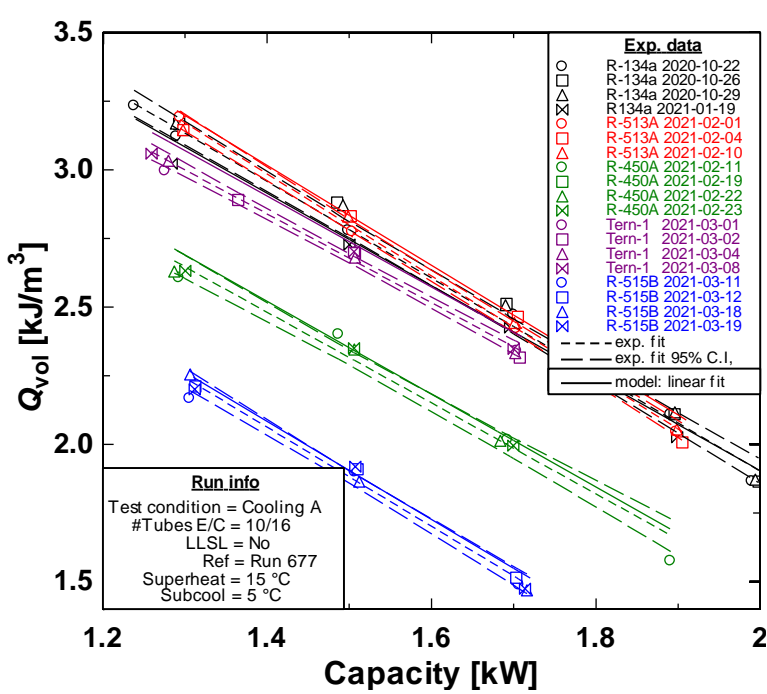

(a)

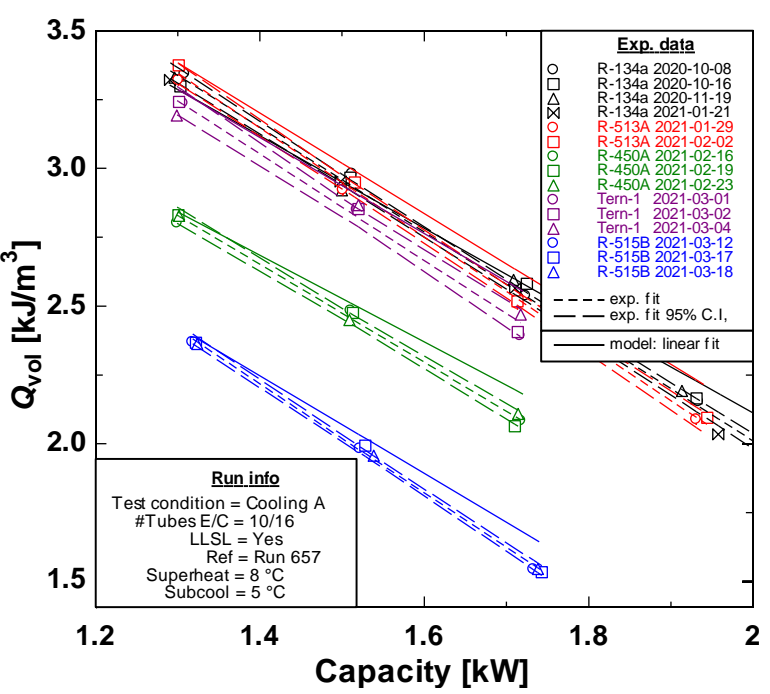

(b)

Figure 3.3-5. Cooling volumetric capacity for HFC-134a and the replacement candidates (a) without LLSL-HX and (b) with LLSL-HX. 


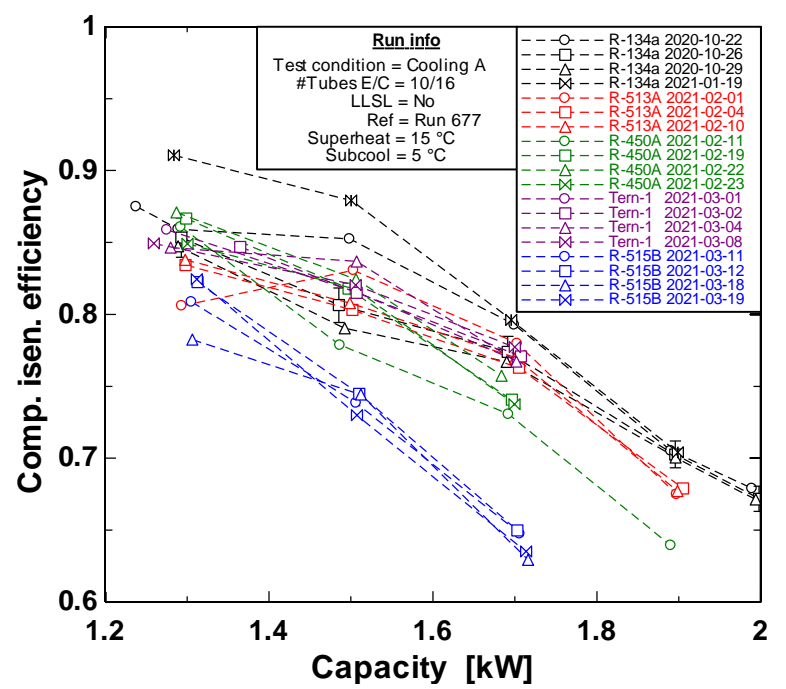

(a)

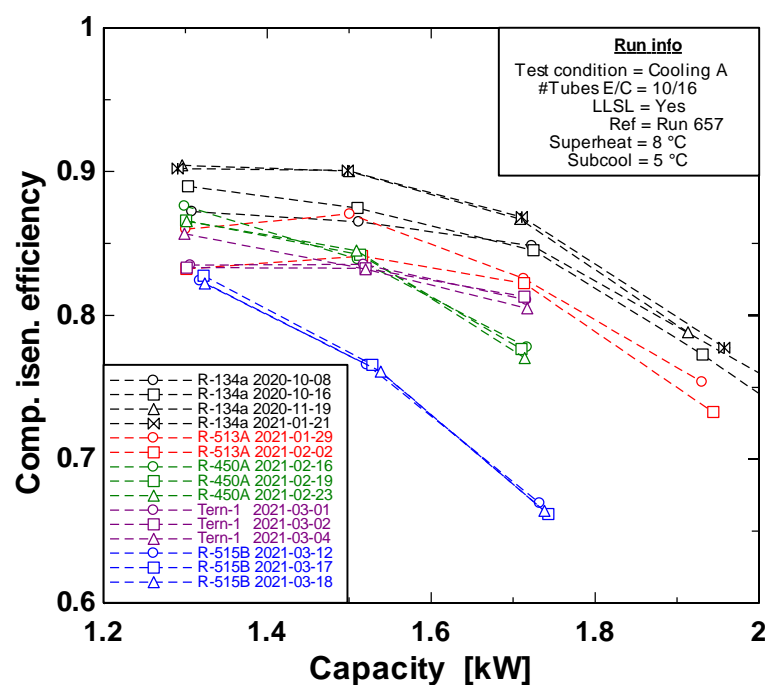

(b)

Figure 3.3-6. Compressor isentropic efficiency (a) without LLSL-HX and (b) with LLSLHX.

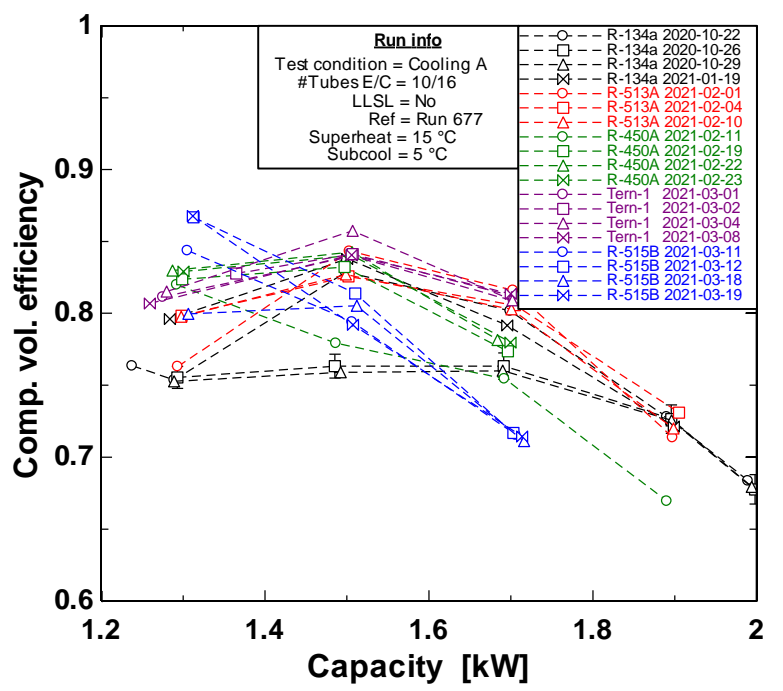

(a)

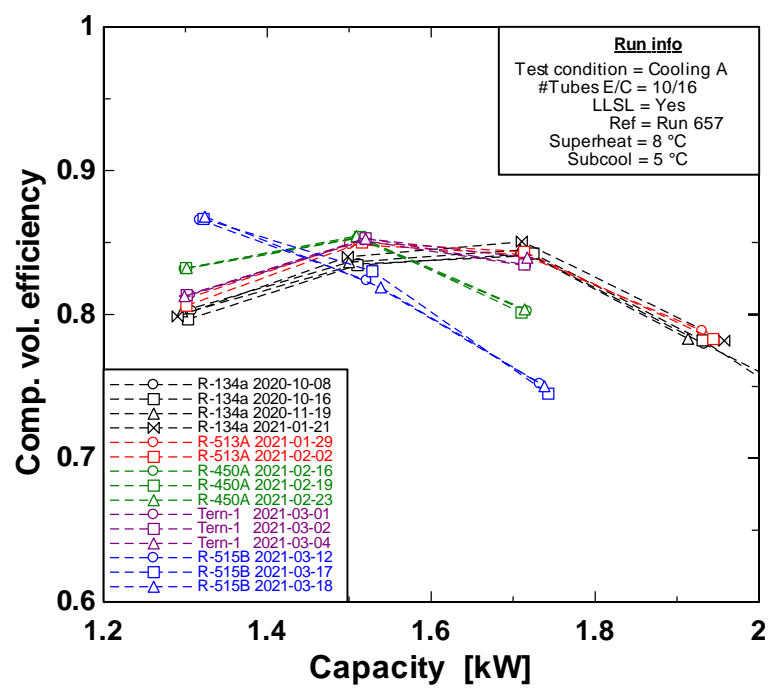

(b)

Figure 3.3-7. Compressor volumetric efficiency (a) without LLSL-HX and (b) with LLSLHX. 


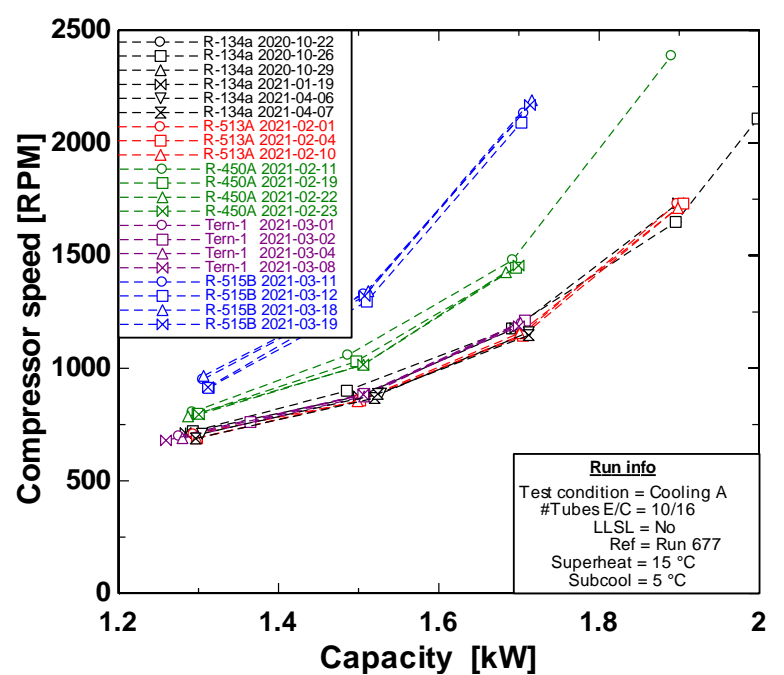

(a)

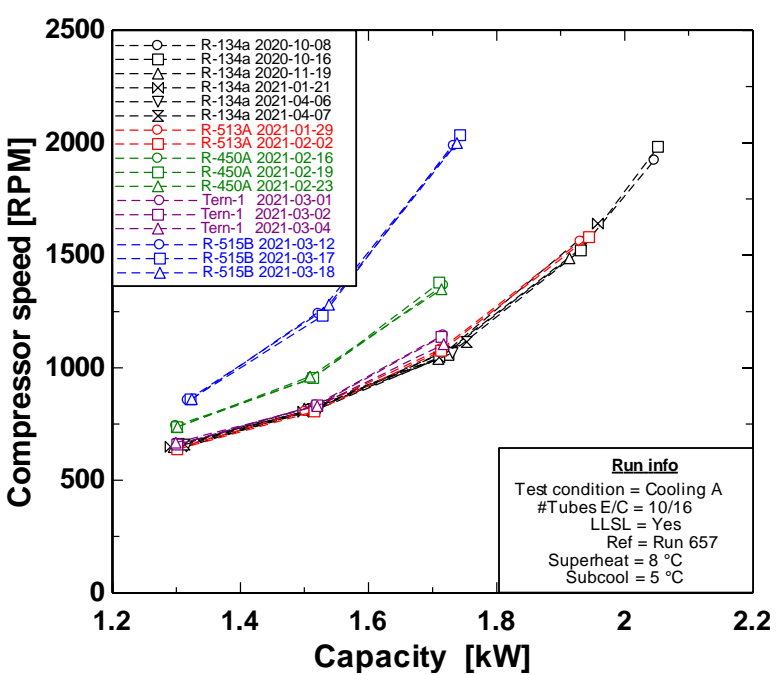

(b)

Figure 3.3-8. Compressor speed (a) without LLSL-HX and (b) with LLSL-HX.

Model Validation. The CYCLE_D-HX simulation COP and $Q_{\text {vol }}$ predictions were also correlated to capacity using linear regression (solid lines on Figure 3.3-4 and Figure 3.3-5). For the tests without the LLSL-HX, the model-predicted values were within the confidence intervals of the experimental results, within about $\pm 1.5 \%$ of the curve fit. The predictions for Tern-1 were an exception, where the model overpredicted the experimental data by about $3 \%$. For the tests with the LLSL-HX, the CYCLE_D-HX predicted the COP and $Q_{\text {vol }}$ within the confidence intervals at $1.3 \mathrm{~kW}$ capacity. At $1.5 \mathrm{~kW}$, the COP and $Q_{\text {vol }}$ were overpredicted by (0 to 3$) \%$, and at $1.7 \mathrm{~kW}$ the COP and $Q_{\text {vol }}$ were overpredicted by ( 1 to 5$) \%$.

Differences between the test data and the CYCLE_D-HX prediction are primarily attributed to the refrigerant heat transfer and pressure drop in the condenser and evaporator, since the thermodynamic property data for the tested fluids are well established and the other hardware performance parameters are input to the model based on each experimental test (including compressor efficiency, LLSL-HX effectiveness, suction \& discharge line pressure drop, HTF temperatures, see Section 3.3.3). Using the R-450A tests without the LLSL-HX as an example, the pressure drop in the condenser and evaporator were predicted within about $\pm 5 \%$ (Figure 3.3-8(a1)), the condenser saturation and outlet temperatures were underpredicted by about $0.3{ }^{\circ} \mathrm{C}$ (Figure 3.3.-8(b1)), and the evaporator saturation and outlet temperatures were overpredicted by about $0.6^{\circ} \mathrm{C}$ (Figure 3.3-8(c1)). Overpredicted evaporator temperatures indicate an underestimation of overall heat transfer resistance between the HTF and the refrigerant. The error is not likely in the thermal resistances of the tube wall conduction and HTF convection, since the heat exchanger size and flow were fixed and empirically determined from the baseline tests and included in the model 'reference case' data. So, we infer the culprit was an underpredicted refrigerant flow-boiling heat transfer resistance. The overpredicted condenser saturation temperature was attributed to an underprediction of refrigerant condensation heat transfer resistance, for similar reasons given for the evaporator. Some of the condenser saturation temperature difference is related to the difference in measured and predicted COP (which determines the amount of heat rejected in 
the condenser), though this effect appears to be small since for R-450A the underpredicted condenser saturation temperature persists despite the nearly exact prediction of COP at $1.5 \mathrm{~kW}$ (Figure 3.3-4(a)).

The R-450A tests with the LLSL-HX highlight the effects of an underprediction in evaporator pressure drop. The evaporator pressure drop is predicted within $\pm 1 \%$ at (1.3 and 1.5) $\mathrm{kW}$ (Figure 3.3-5(a2)), and the evaporator saturation temperature was overpredicted by $0.6{ }^{\circ} \mathrm{C}$ (Figure 3.3-5(b2)). In contrast, at $1.7 \mathrm{~kW}$ capacity the pressure drop was underpredicted by $10 \%$, and the evaporator saturation temperature was overpredicted by $1.2{ }^{\circ} \mathrm{C}$. Pressure drop in the evaporator causes a reduction in saturation temperature that is unfavorable to countercurrent heat exchange, requiring a lower saturation temperature to drive the heat transfer. So underpredicting pressure drop results in overpredicted saturation temperature and a subsequent overprediction of COP (Figure 3.3-4(b)) as expected from the Carnot efficiency of a heat pump operating with a underpredicted temperature lift. This overprediction of COP occurred despite an overprediction of condenser pressure drop of ( 0 to 15) $\%$, indicating the evaporator pressure drop is more important for determining the cycle COP.

In summary, the CYCLE_D-HX model predicted the same relative COP and $Q_{\text {vol }}$ ranking as the experimental data, giving confidence to the HFC-134a replacement candidate screening performed in the limited-scope project. All four low-GWP candidates are acceptable for testing in the ECU as none had significant deviations from modeled performance, excessive discharge temperatures, or other hardware-related problems. 


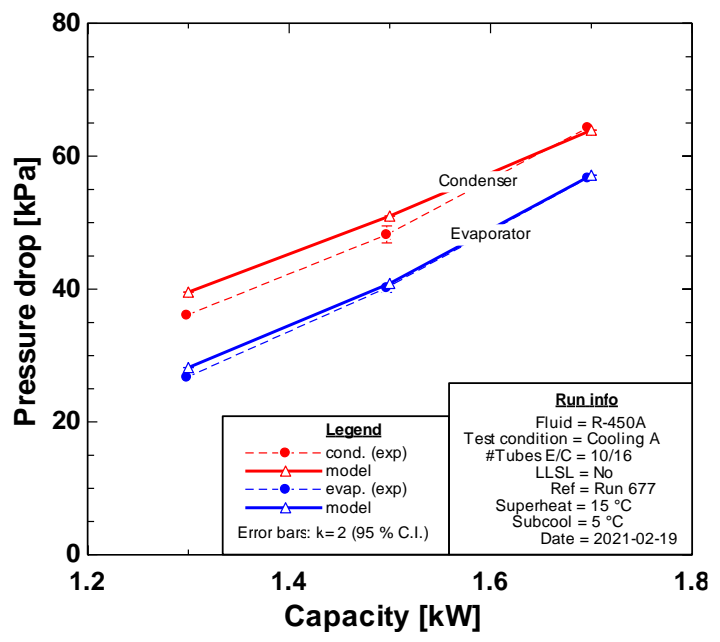

(a1) - without LLSL-HX

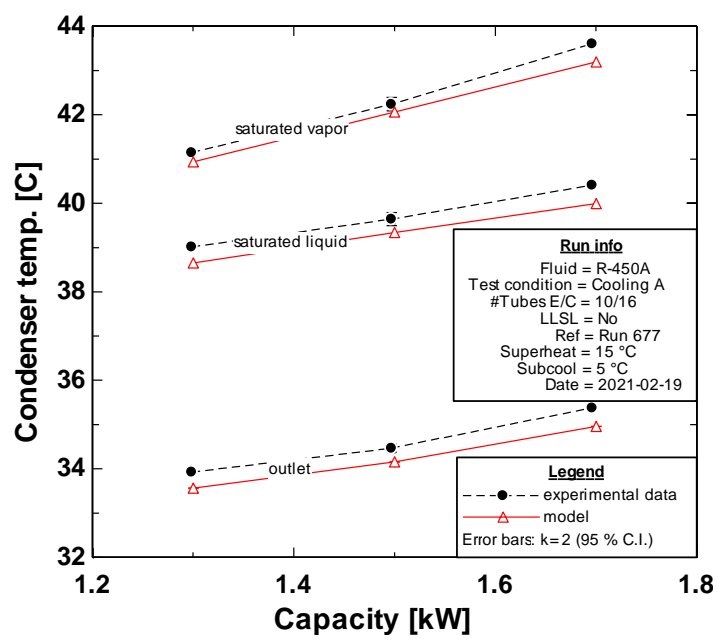

(b1) - without LLSL-HX

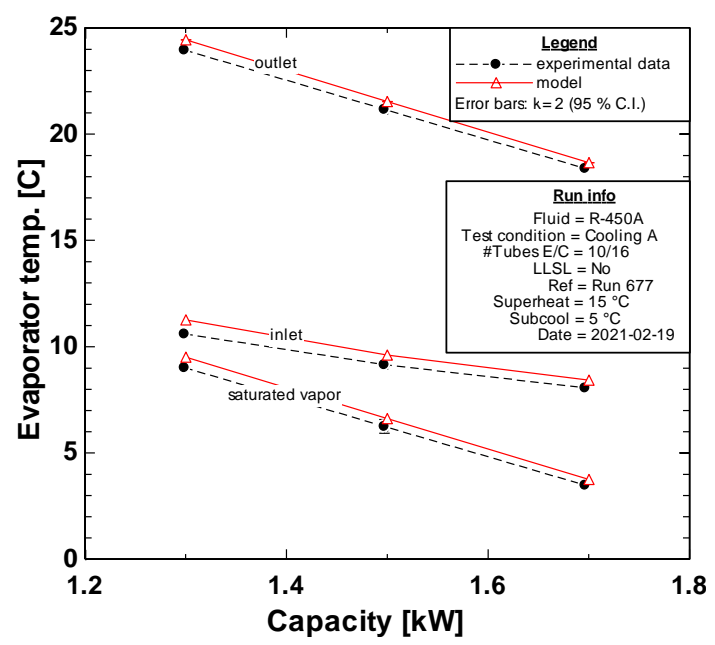

(c1) - without LLSL-HX

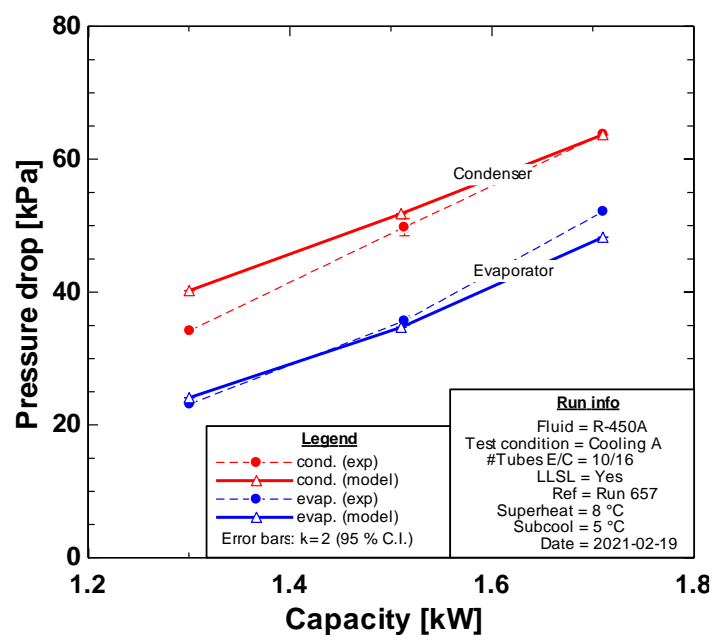

(a2) - with LLSL-HX

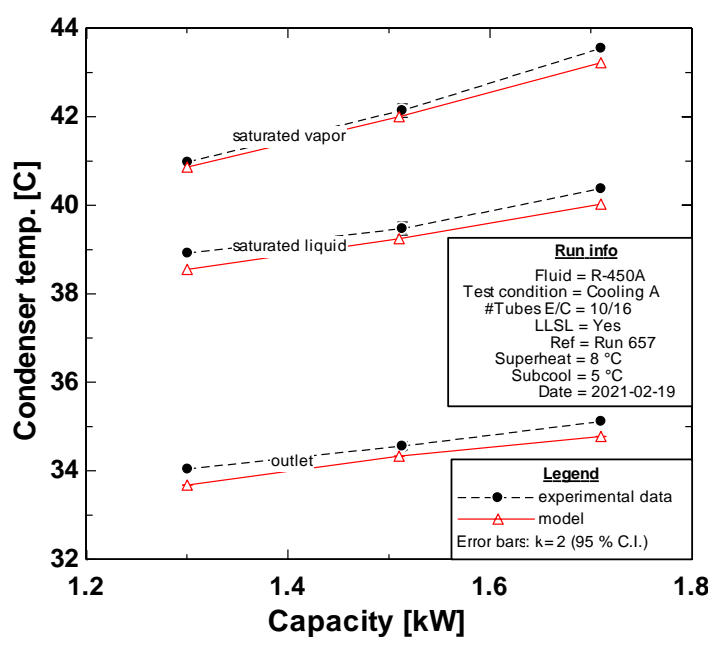

(b2) - with LLSL-HX

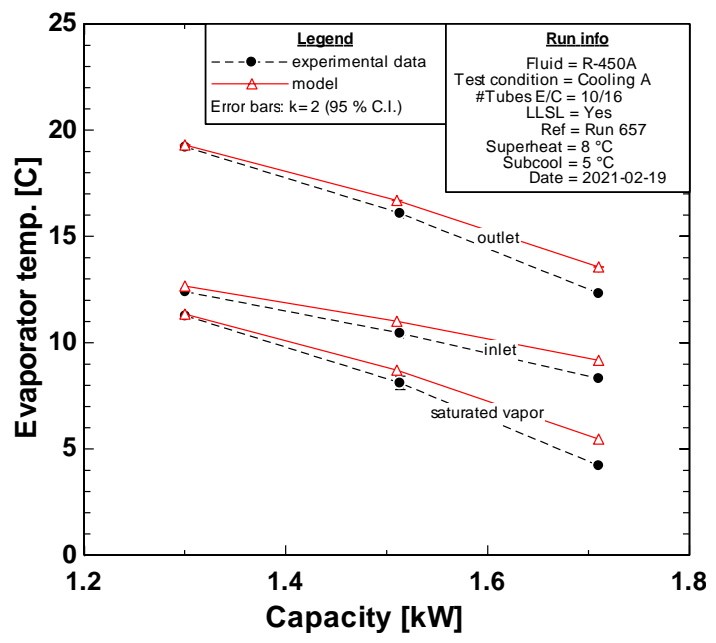

(c2) - with LLSL-HX

Figure 3.3-9. R-450A measurement and model prediction of: (a) evaporator and condenser pressure drop, (b) evaporator temperatures, and (c) condenser temperatures. 


\subsection{Task 4: Refrigerant Forced-Convection Heat-Transfer Testing}

Measured refrigerant flow boiling heat-transfer coefficients within a micro-fin tube are presented in this section for three low-GWP HFC/HFO refrigerant blends, R-513A, R-450A, and R-515B. A new correlation of the flow boiling heat-transfer coefficient, including data for previously measured HFC- $134 \mathrm{a}^{57}$, is presented. The micro-fin tube is a good choice for experimentation because this type of enhancement is ubiquitous in unitary equipment. Measurements were made to validate and improve the existing NIST evaporation correlation to include HFC/HFO blends for application in heat exchanger and air-conditioning system simulation tools.

\subsubsection{Test Apparatus}

Figure 3.4-1 shows a sketch of the experimental apparatus used to establish and measure convective boiling heat transfer coefficients. The experimental test facility consisted of two main systems: the refrigerant loop and the water loop. The refrigerant flow rate, pressure, and quality were fixed at the inlet to the test section. The water flow rate and the inlet temperature were fixed to establish the overall refrigerant quality change in the test section. The water temperature drop, the tube wall temperature, the refrigerant temperatures, pressures, and pressure drops were measured at several axial locations along the test section. These measurements were used to calculate the local heat-transfer coefficient for the microfin tube.

The test section consisted of a pair of $3.34 \mathrm{~m}$ long, horizontal tubes connected by a U-bend. A fixed test pressure was maintained by balancing the refrigerant duty between the subcooler, the test section, the preheater and the condensers. A magnetically coupled gear pump delivered the test refrigerant to the entrance of the test section as saturated, near zero quality liquid. Another magnetically coupled gear pump supplied a steady flow of water to the annulus of the test section. The inlet temperature of the water loop was held constant for each test with a water-chilled heat exchanger and variable electric heaters. The refrigerant and water flow rates were controlled by varying the pump speeds using frequency inverters. Redundant flow rate measurements were made with Coriolis flowmeters and with turbine flowmeters for both the refrigerant and water sides. 


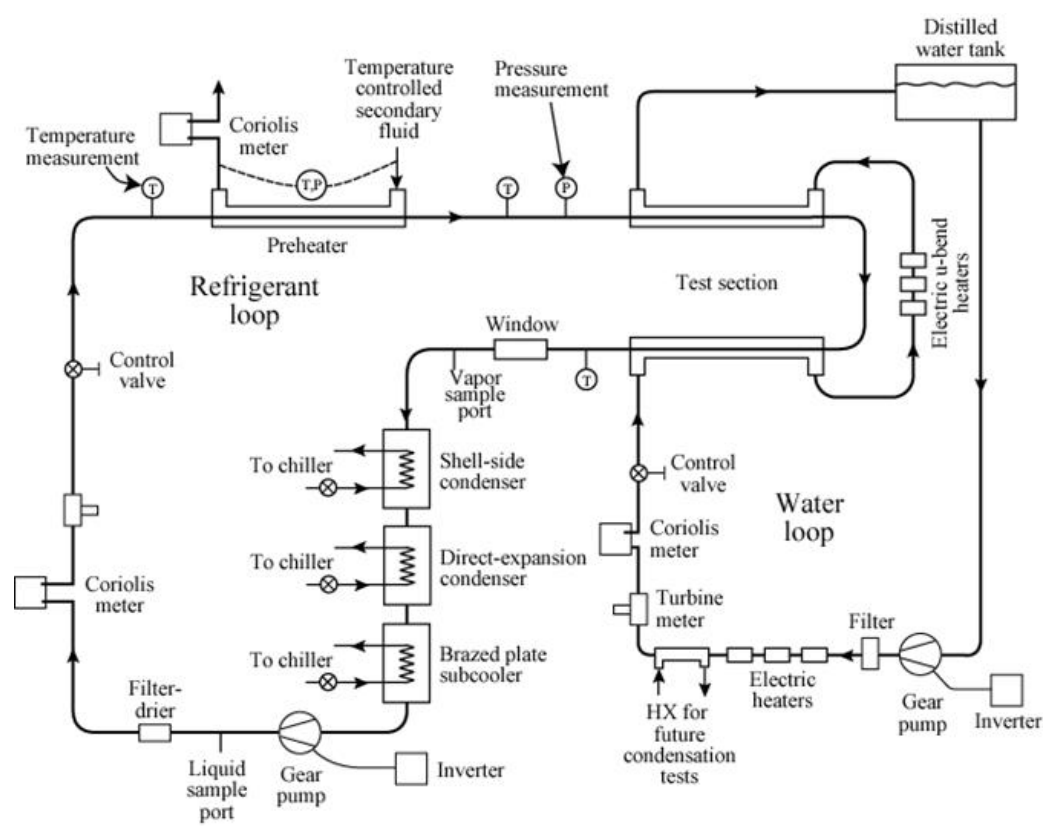

Figure 3.4-1. Schematic of flow boiling test apparatus.

Figure 3.4-2. shows a cross section of the test section with a detail of the micro-fin tube geometry. The test refrigerant flowed inside a micro-fin tube, while distilled water flowed either in parallel flow or counterflow to the refrigerant in the annulus that surrounded the micro-fin tube. Conducting some tests in parallel flow and others in counterflow (as shown in Figure 3.4-3.) produced a broad range of heat fluxes at both low and high flow qualities. The annulus gap was $2.2 \mathrm{~mm}$, and the micro-fin tube wall thickness was $0.3 \mathrm{~mm}$. The micro-fin tube had 60, $0.2 \mathrm{~mm}$ high fins that rifled down the axis of the tube at a helix angle $(\alpha)$ of $18^{\circ}$ with respect to the tube axis. For this geometry, the cross-sectional flow area was $60.8 \mathrm{~mm}^{2}$, giving an equivalent smooth diameter $\left(D_{\mathrm{e}}\right)$ of $8.8 \mathrm{~mm}$. The root diameter of the micro-fin tube was $8.91 \mathrm{~mm}$. The inside-surface area per unit length of the tube was estimated to be $44.6 \mathrm{~mm}$. The hydraulic diameter $\left(D_{\mathrm{h}}\right)$ was measured with a polar planimeter from a scaled drawing of the tube cross section and determined to be approximately $5.45 \mathrm{~mm}$. The ratio of the inner surface area of the micro-fin tube to the surface area of a smooth tube of the same $D_{\mathrm{e}}$ was 1.6. 

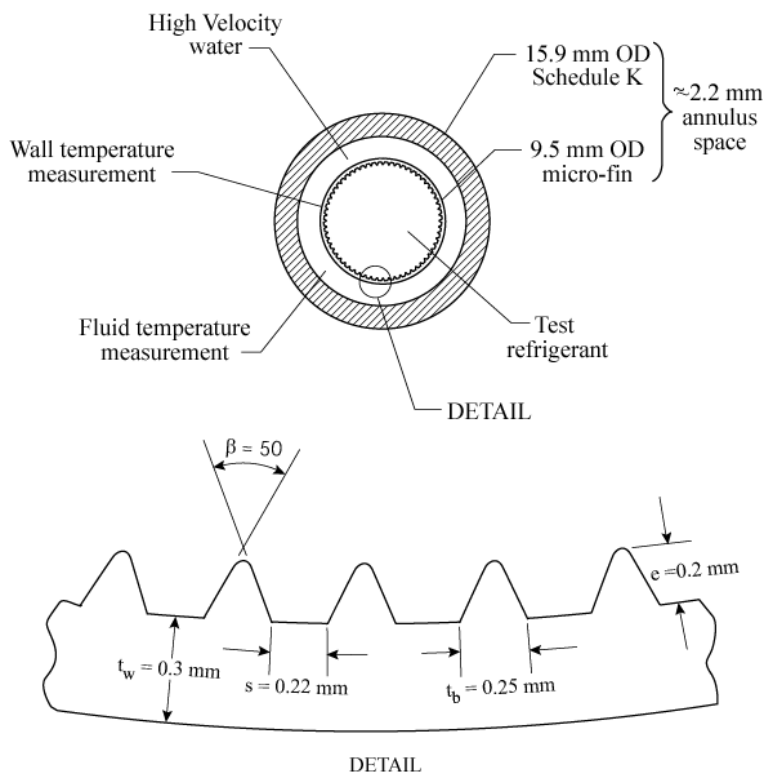

Figure 3.4-2. Cross section of flow-boiling test section.

Figure 3.4-3. provides a detailed schematic of the test section. The annulus was constructed by connecting a series of tubes with 14 pairs of stainless-steel flanges. This construction permitted the measurement of both the outer micro-fin wall temperature and the water temperature drop as discussed in the following two paragraphs. The design also avoided abrupt discontinuities such as unheated portions of the test section and tube-wall "fins" between thermopile ends.

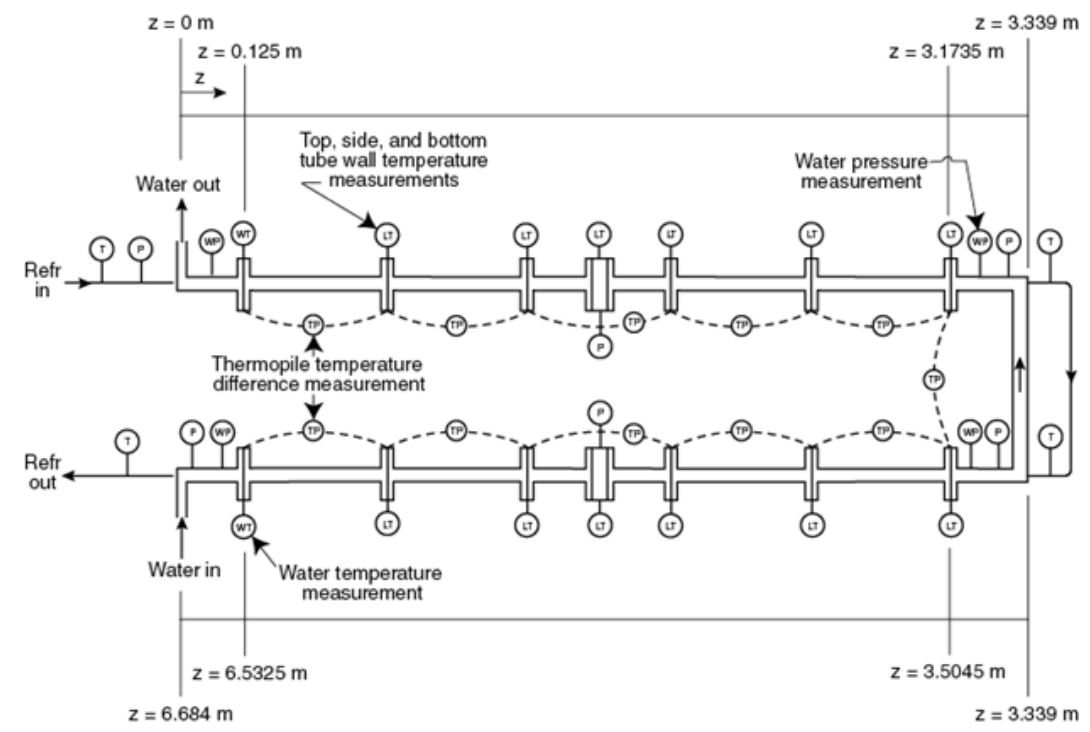

Figure 3.4-3. Detailed schematic of test section (counterflow)

Figure 3.4-3. shows that thermocouple wires pass between 12 of the gasketed flange pairs to measure the refrigerant-tube wall temperature at ten locations on the top, side, and bottom of the tube wall. These locations were separated by $0.6 \mathrm{~m}$ on average, and they were located near the intersection of the shell flanges. In addition to these, thermocouples were also 
mounted next to the pressure taps near the middle of each test section length. The thermocouple junction was soldered to the outside surface and was sanded to a thickness of approximately $0.5 \mathrm{~mm}$. The leads were strapped to a thin non-electrically-conducting epoxy layer on the wall for a distance of $14.3 \mathrm{~mm}$ before they passed between a pair of the shell flanges. The wall temperature was corrected for a heat flux dependent fin effect. The correction was typically $0.05 \mathrm{~K}$. Figure $3.4-3$. also shows that a chain of thermopiles was used to measure the water temperature drop between each flange location. Each thermopile consisted of ten thermocouples in series, with the ten junctions at each end evenly spaced around the circumference of the annulus. Because the upstream junctions of one thermopile and the downstream junctions of another enter the annulus at the same axial location (except at the water inlet and outlet), the junctions of the adjacent piles were alternated around the circumference. A series of Teflon half-rings attached to the inner refrigerant tube centered the tube in the annulus. The half-rings were circumferentially baffled to mix the water flow. Mixing was further ensured by a turbulent water Reynolds number ${ }^{58}$.

As shown in Figure 3.4-3., six refrigerant pressure taps along the test section allowed the measurement of the upstream absolute pressure and five pressure drops along the test section. Two sets of two water pressure taps were used to measure the water pressure drop along each tube. Also, a sheathed thermocouple measured the refrigerant temperature at each end of the two refrigerant tubes, with the junction of each centered radially. Only the thermocouple at the inlet of the first tube was used in the calculations. The entire test section was wrapped with $5 \mathrm{~cm}$ of foam insulation to minimize heat transfer between the water and the ambient.

\subsubsection{Data Analysis and Correlation Development}

The convective boiling heat-transfer coefficient based on the actual inner surface area $\left(h_{2 \phi}\right)$ was calculated as:

$$
h_{2 \phi}=\frac{q^{\prime \prime}}{T_{\mathrm{w}}-T_{\mathrm{s}}}
$$

where the measured wall temperatures $\left(T_{\mathrm{w}}\right)$ were fitted to their axial position (distance along the test section) to reduce the uncertainty in the measurement.

The average estimated expanded uncertainty of the wall temperature fit for all the measurements at the $95 \%$ confidence level, was approximately $0.42 \mathrm{~K}$ and $0.36 \mathrm{~K}$, for the counterflow and the parallel flow data, respectively. The median of the uncertainty in $T_{\mathrm{w}}$ was approximately $0.4 \mathrm{~K}$.

The water temperature $\left(T_{\mathrm{f}}\right)$ was determined from the measured temperature change obtained from each thermopile and the inlet water temperature measurement. The water temperature gradient $\left(\mathrm{d} T_{\mathrm{f}} / \mathrm{d} z\right)$ was calculated with second-order finite difference equations using the measured water temperatures and their locations along the tube length $z$. The water temperature gradients were then fitted with respect to the tube length. The measured water temperatures typically agreed with the integrated fit of the water temperature gradient to within $0.2 \mathrm{~K}$. 
The fitted, local, axial water temperature gradient $\left(\mathrm{d} T_{\mathrm{f}} / \mathrm{d} z\right)$, the measured water mass flow rate $(\dot{m})$, and the properties of the water were used to calculate the local heat flux $\left(q^{\prime \prime}\right)$ to the micro-fin tube based on the actual inner surface area:

$$
q^{\prime \prime}=\frac{\dot{m}_{\mathrm{f}}}{p}\left(c_{\mathrm{p}_{\mathrm{f}}} \frac{\mathrm{d} T_{\mathrm{f}}}{\mathrm{d} z}+v_{\mathrm{f}} \frac{\mathrm{d} P_{\mathrm{f}}}{\mathrm{d} z}\right)
$$

where $p$ is the wetted perimeter of the inside of the micro-fin tube. The specific heat $\left(c_{\mathrm{pf}}\right)$ and the specific volume $\left(v_{f}\right)$ of the water were calculated locally as a function of the water temperature. The water pressure gradient $\left(\mathrm{d} P_{\mathrm{f}} / \mathrm{d} z\right)$ was linearly interpolated between the pressure taps to the location of the wall thermocouples. The pressure gradient term was typically less than $3 \%$ of the temperature gradient term. The heat flux obtained by Eq. (3.42 ) was reduced by the amount of heat lost to the surroundings. The heat loss to the surroundings was obtained by calibration of single-phase heat-transfer tests, and it was based on the temperature difference between the room and the test fluid. Typically, the heat loss correction was less than a $0.1 \%$ of that obtained from Eq. (3.4-2). The relative uncertainty of the heat flux measurement was less than $40 \%$ of the measured value, while the average uncertainty for the counterflow and the parallel flow data was approximately $7 \%$ and $20 \%$ of the measured value, respectively.

The local Nusselt number $(\mathrm{Nu})$ was calculated using the hydraulic diameter and the heattransfer coefficient based on the actual inner surface area of the tube as:

$$
\mathrm{Nu}=\frac{h_{2 \phi} D_{h}}{k_{l}}
$$

The uncertainty of $\mathrm{Nu}$ was between roughly $10 \%$ and $40 \%$. Measurements of $\mathrm{Nu}$ with uncertainties greater than $30 \%$ were discarded. Reduction in the uncertainty can be achieved with repeat measurements for the same operating conditions. However, repeat measurements are difficult to obtain due to the chaotic nature of two-phase flow and the many fixed parameters that need to be matched between measurements.

The 432 measured local convective boiling Nusselt numbers $(\mathrm{Nu})$ for R-515B, R-450A, R513A, and HFC-134a were compared to the pure-refrigerant (single component) version of the Hamilton et al. correlation ${ }^{59}$ :

$$
\mathrm{Nu}_{\mathrm{p}}=482.18 \operatorname{Re}^{0.3} \operatorname{Pr}^{\mathrm{C}_{1}}\left(\frac{P_{\mathrm{s}}}{P_{\mathrm{c}}}\right)^{\mathrm{C}_{2}} \mathrm{Bo}^{\mathrm{C}_{3}}\left(-\log _{10} \frac{P_{\mathrm{s}}}{P_{\mathrm{c}}}\right)^{\mathrm{C}_{4}} M_{\mathrm{w}}{ }^{\mathrm{C}_{5}}
$$

where

$$
\begin{aligned}
& C_{1}=0.51 x_{q} \\
& C_{2}=5.57 x_{q}-5.21 x_{q}^{2} \\
& C_{3}=0.54-1.56 x_{q}+1.42 x_{q}^{2} \\
& C_{4}=-0.81+12.56 x_{q}-11.00 x_{q}^{2} \\
& C_{5}=0.25-0.035 x_{q}^{2}
\end{aligned}
$$


Here, the all-liquid Reynolds number (Re), the Boiling number (Bo), the liquid Prandtl number $(\operatorname{Pr})$, the reduced pressure $\left(P_{\mathrm{s}} / P_{\mathrm{c}}\right)$, and the quality $\left(x_{\mathrm{q}}\right)$ are all evaluated locally at the saturation temperature. The all-liquid Reynolds number and the Nusselt number are based on the hydraulic diameter $\left(D_{\mathrm{h}}\right)$. The Nusselt number is also based on the actual inner surface area of the tube.

Figure 3.4-4 plots the present measurements versus predicted values of the Nusselt number for R-515B, R-450A, and R-513A. Previously made measurements for R-450A ${ }^{60}$, R-513A ${ }^{57}$ and $\mathrm{HFC}-134 \mathrm{a}^{57}$ are also included in the comparison. The Hamilton et al. correlation ${ }^{59}$ predicts approximately $43 \%$ measurements to within $\pm 20 \%$.

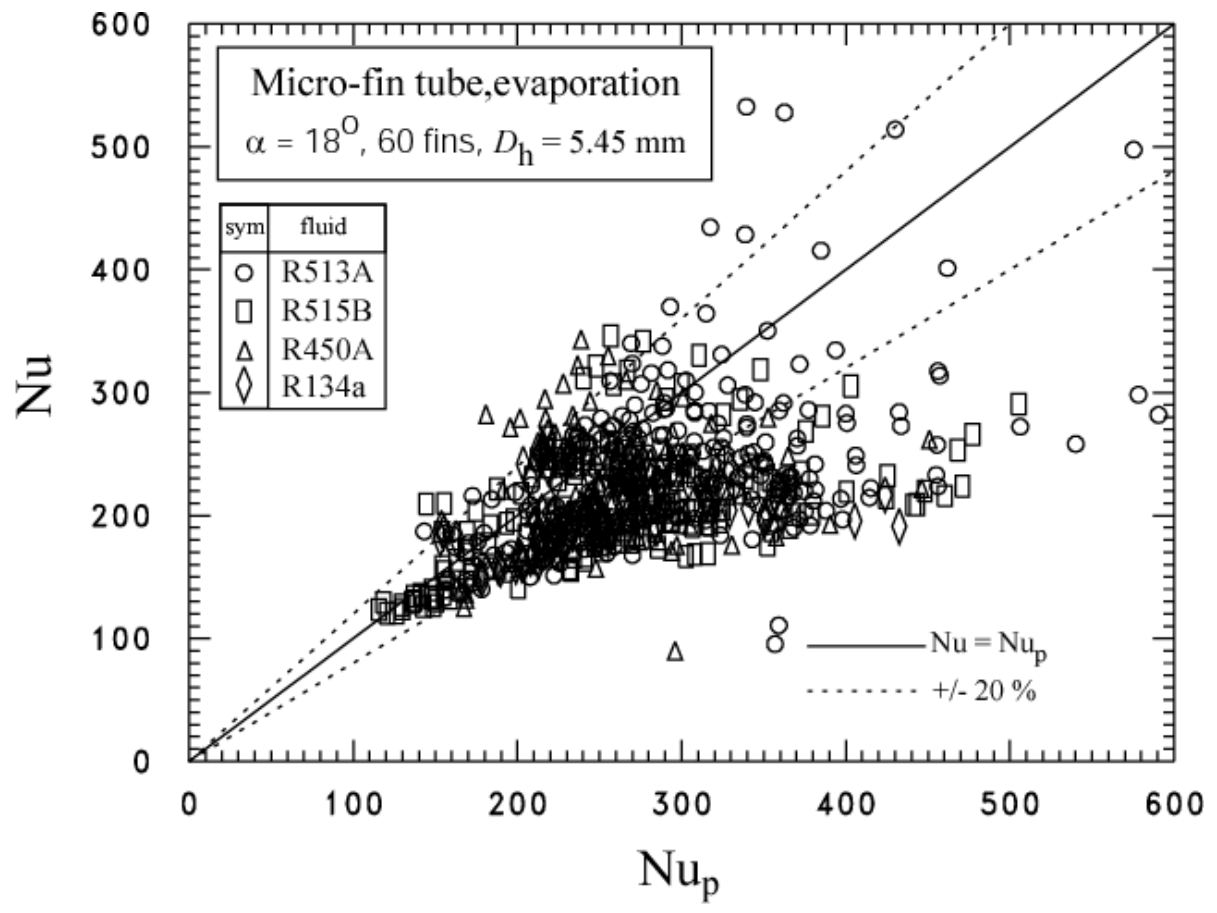

Figure 3.4-4. Comparison between measured Nusselt numbers and those predicted by the Hamilton et al. correlation ${ }^{59}$.

A new correlation was developed to more accurately represent the current flow-boiling heattransfer measurements:

$$
\mathrm{Nu}_{\mathrm{p}}=242.5 \mathrm{Re}^{0.26\left(1-x_{q}\right)} \mathrm{Bo}^{0.28} \mathrm{~B}_{\mathrm{nd}}^{-0.61 x_{q}}
$$

The new correlation (Eq. 3.4-5) predicts approximately $71 \%$ of the measured convective boiling Nusselt numbers for R-515B, R-450A, R-513A, and HFC-134a to within approximately $\pm 20 \%$ (Figure 3.4-5.). $\mathrm{B}_{\mathrm{nd}}$ is the dimensionless Bond number ${ }^{61}$, which includes fin geometry parameters and the surface tension as defined in the Nomenclature. The correlation is valid for Re between 1000 and 14000, Bo between 0.000002 and 0.001 , and $\mathrm{B}_{\mathrm{nd}}$ between 0.002 and 0.05. Equation (3.4-4) was developed with data where the refrigerant reduced temperature ranged between approximately 0.71 and 0.94 . 


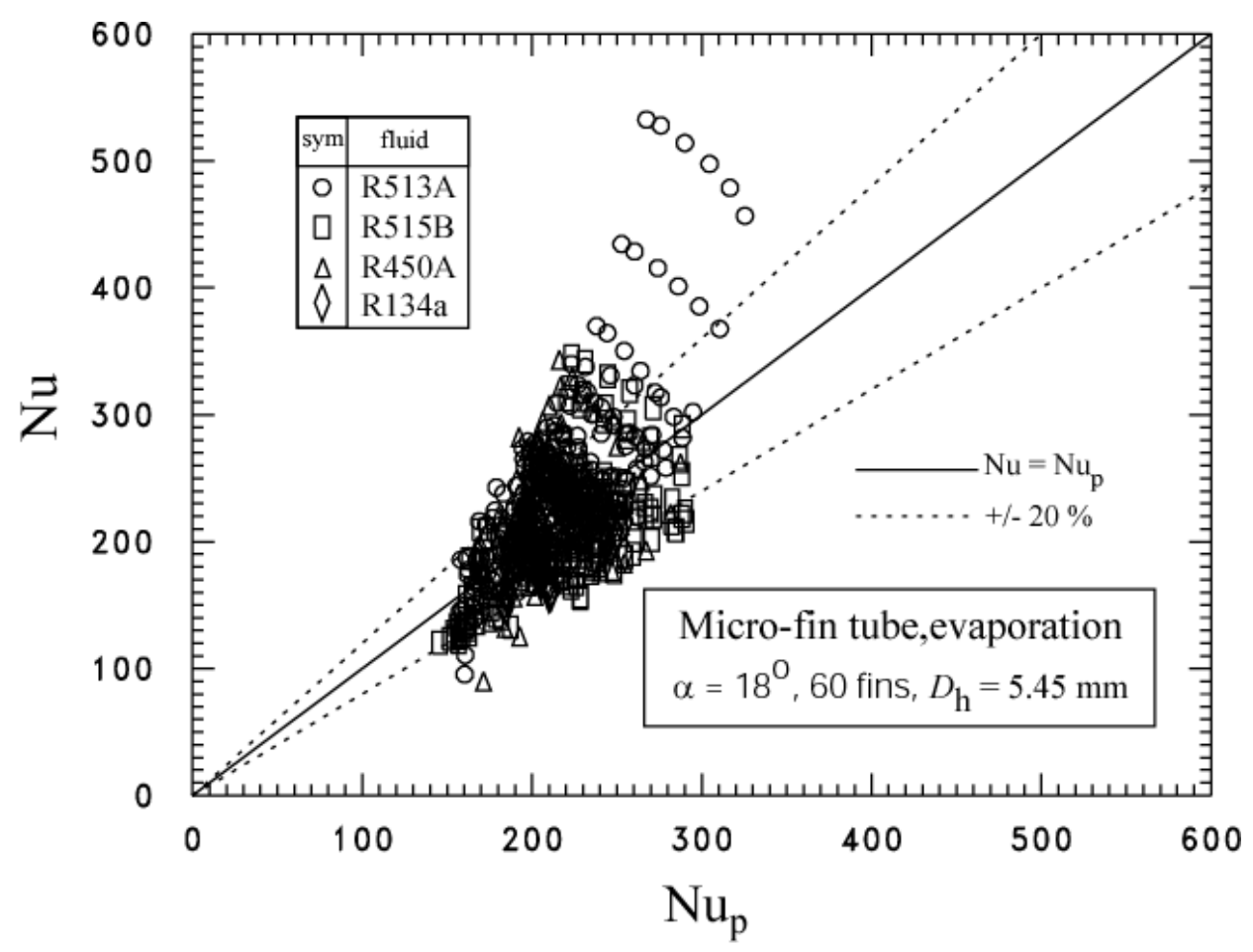

Figure 3.4-5. Comparison between measured Nusselt numbers and those predicted by the new correlation given by Eq. (3.4-5). 


\subsection{Task 5: Selection of Final Blends for Testing in Military ECU}

\subsubsection{Evaluation of "New" Fluids}

The project plan calls for experimental evaluation of four blends in the MBHP (discussed earlier) to inform the selection of three 'best' blends for testing in the ECU. While cycle simulation results from the limited-scope project gave us a good basis for selecting 'best' blends, we continually monitored technical developments in low-GWP fluids to make sure that we did not overlook any promising new fluids.

A review of ASHRAE Standard 34 shows three new single-compound refrigerants that were classified since the completion of the analysis for the limited-scope project in 2017:

- HFO-1336mzz(E) $\left(\mathrm{CF}_{3} \mathrm{CH}=\mathrm{CHCF}_{3}\right)$, safety classification $\mathrm{A} 1, \mathrm{NBP}=7.4{ }^{\circ} \mathrm{C}$

- HFO-1132a $\left(\mathrm{CF}_{2}=\mathrm{CH}_{2}\right)$, safety classification $\mathrm{A} 2, \mathrm{NBP}=-83{ }^{\circ} \mathrm{C}$

- R-13I1 $\left(\mathrm{CF}_{3} \mathrm{I}\right)$, safety classification $\mathrm{A} 1, \mathrm{NBP}=-21.9^{\circ} \mathrm{C}$

As indicated by its relatively high NBP, HFO-1336mzz(E) is a low-pressure refrigerant and not suitable for application in medium-pressure AC equipment, even as a blend component. The very low NBP of HFO-1132a, on the other hand, makes it unsuitable at the other extreme; in a blend it would result in high pressures and a significant temperature glide. HFO-1132a is also flammable.

Consideration of $\mathrm{CF}_{3} I$. The NBP of $\mathrm{CF}_{3} \mathrm{I}$, on the other hand, is close to that of HFC-134a $\left(-26.1^{\circ} \mathrm{C}\right)$, which makes $\mathrm{CF}_{3} \mathrm{I}$, thermodynamically at least, a fluid warranting consideration. Its GWP is also very low; a relatively old study characterized its GWP as "likely to be very small, less than 5." ${ }^{\circ 2}$ The appeal of $\mathrm{CF}_{3} \mathrm{I}$ is further increased by its flame-suppression characteristics. A drawback of $\mathrm{CF}_{3} \mathrm{I}$ is its reactivity, which would require the application of proprietary chemical stabilizers not available on the open market.

We spent a considerable analytical effort to formulate, within the constraints of publicly available data, a state-of-the-art representation of thermodynamic properties of both the pure fluid and blends containing $\mathrm{CF}_{3} \mathrm{I}$. This effort was summarized by Bell and McLinden. ${ }^{63} \mathrm{We}$ also added $\mathrm{CF}_{3} \mathrm{I}$ to the screening developed under the limited-scope project in a search for the best performing $\mathrm{CF}_{3} \mathrm{I}$-based blends. Although the $\mathrm{COP}$ and volumetric capacity of the pure $\mathrm{CF}_{3} \mathrm{I}$ was poor compared to HFC-134a, these simulations identified some blends of $\mathrm{CF}_{3} \mathrm{I}$ with HFC-152a and HFC-32 as having potentially favorable performance. The high-performing blends, however, contained a low fraction of $\mathrm{CF}_{3} \mathrm{I}$. Specifically, for R-152a/CF $\mathrm{I}$ blends a mole fraction of HFC-152a of 0.80 (mass fraction of 0.57) yielded the same COP as HFC134a. For blends with HFC-32, the corresponding mole fraction was 0.75 (mass fraction of 0.32 ). This led us to question whether such blends were still nonflammable.

The flame inhibition characteristics of $\mathrm{CF}_{3} \mathrm{I}$ have been studied. We could find no information on the specific mixture of HFC-152a/CF 3 , but Yang et al. ${ }^{64}$ report that $\mathrm{CF}_{3} \mathrm{I}$ is substantially more effective than HFC-125 in inhibiting the flammability of HFC-32 (a minimum concentration of $0.0536 \mathrm{~kg} \cdot \mathrm{m}^{-3}$ for $\mathrm{CF}_{3} \mathrm{I}$ versus $0.0984 \mathrm{~kg} \cdot \mathrm{m}^{-3}$ for $\left.\mathrm{HFC}-125\right)$. We can use information on the blend R-410A (the blend of HFC-32 and HFC-125 at a mass composition of (50.0/50.0) and the blend R-466A (the blend of HFC-32, HFC-125, and $\mathrm{CF}_{3} \mathrm{I}$ at a mass 
composition of (49.0/11.5/39.5)) to formulate an informed guess on the flammability of HFC-152a/CF $\mathrm{I}$ ) with the following reasoning. Both R-410A and R-466A are classified as A1 (i.e., nonflammable) and contain about $50 \%$ of the flammable HFC-32 with a with a $50 \%$ content of flame suppressing agents (i.e., HFC-125 and/or $\mathrm{CF}_{3} \mathrm{I}$ ). In the detailed report of flammability testing contained in the application to the Standard 34 committee $^{65}$ it is seen that R-466A exhibits a flame angle as large as $40^{\circ}$ in the ASTM E681 test protocol under some conditions. This is "not flammable" under the E681 protocol (which defines "flammable" as a flame angle of $90^{\circ}$ or larger), but it does indicate that R-466A was formulated close to the border of flammability. Given that HFC-152a is more flammable than HFC-32, a $\mathrm{CF}_{3} \mathrm{I}$ fraction greater than $50 \%$ would likely be required to suppress flammability, i.e., compositions that showed lower performance in the simulations.

There are also stability and toxicity concerns around $\mathrm{CF}_{3} \mathrm{I}$. Although having a safety classification of $\mathrm{A} 1, \mathrm{CF}_{3} \mathrm{I}$ has a relatively low "refrigerant concentration limit" (RCL) of 2000 ppm (i.e., a concentration in air of $0.2 \%$ by volume). ${ }^{66}$ This compares to an RCL of 50000 ppm for HFC-134a. ${ }^{12}$ The RCL considers a range of hazards and is "intended to reduce the risks of acute toxicity, asphyxiation, and flammability hazards in normally occupied, enclosed spaces." 12 The RCL impacts the maximum charge allowed in a particular system. We also note that in a review of the toxicity of $\mathrm{CF}_{3} \mathrm{I}$, the National Research Council recommended that it be used (as a fire suppressant) only in unoccupied spaces. ${ }^{67}$ The chemical stability of the $\mathrm{CF}_{3} \mathrm{I}$ molecule is substantially lower than typical HFC refrigerants, and it would require stabilizers for use in a refrigeration system. Such stabilizers have been developed, but they are proprietary. NIST engaged with a refrigerant manufacturer that was pursuing the development of $\mathrm{CF}_{3} \mathrm{I}$-containing refrigerant blends, such as $\mathrm{R}-466 \mathrm{~A}$, in an attempt to obtain samples of $\mathrm{CF}_{3} \mathrm{I}$ and the necessary stabilizers. These negotiations were not successful.

Finally, a recent "roundtable discussion" published in ASHRAE Journal ${ }^{68}$ illustrates that industry disagreed about the future of $\mathrm{CF}_{3} \mathrm{I}$ and $\mathrm{CF}_{3} \mathrm{I}$ blends. One company stated that "An emerging nonflammable (ASHRAE class A1) <750 GWP candidate, R-466A, is under heavy consideration by a number of manufacturers." And that "R-466A would be an ideal interim solution ... until further innovation could lead to even lower GWP nonflammable alternatives." In contrast, a second company stated that R-466A was "in our opinion, questionable for stability and for compatibility with materials as a result of potential acid formation. This can lead to reliability, durability and performance problems over time." And "No major North American equipment or compressor manufacturers have announced or shared that they are developing equipment and component parts around this refrigerant. This suggests it is not presently viewed as a viable candidate."

Taking all of the above in its totality, we conclude that it is not feasible to include $\mathrm{CF}_{3} \mathrm{I}$ at this point in time. The ultimate utility of $\mathrm{CF}_{3} \mathrm{I}$ as a refrigerant remains an open research question.

Consideration of $H F O-1132(E)$. In addition to the fluids noted above that were added to the ASHRAE standard another isomer of difluoroethene is currently being studied for use as a refrigerant:

- HFO-1132(E) (trans-1,2-difluoroethene), safety classification not assigned, NBP $=-53{ }^{\circ} \mathrm{C}$ 
The $-53{ }^{\circ} \mathrm{C}$ boiling point of HFO-1132(E) is similar to that of R-410A $\left(-51.5{ }^{\circ} \mathrm{C}\right)$, making it of high commercial interest. Limited data on this fluid are now becoming available. ${ }^{69} 70$ HFO-1132(E) was one of the fluids identified in the comprehensive screening of McLinden et al. ${ }^{71}$ that was in the category of "novel molecules" for which "few data could be found." The analysis of McLinden et al. was based on a predicted critical temperature of $370.5 \mathrm{~K}$, which resulted in a low volumetric capacity relative to R-410A (which was the baseline for that study); this contrasts the recently measured critical temperature of $348.8 \mathrm{~K},{ }^{70}$ which is similar to R-410A. Much of the research on HFO-1132(E) remains proprietary, making detailed simulations of its performance impossible at this time. Furthermore, it is flammable (likely ASHRAE classification of "2") and not commercially available. For these reasons, HFO-1132(E) was dropped from further consideration in this project.

\subsubsection{Selection of Four Blends for Testing in Mini-Breadboard Heat Pump}

The cycle simulation results from the limited-scope project were the primary basis for selecting 'best' blends for testing (Table 3.5-1). As noted in the previous section, no new fluids were identified that could be considered as viable blend components.

The criteria for blend selection consisted of the following parameters: (1) non-flammability $(\bar{\Pi}<0)$; (2) minimum GWP; (3) maximum coefficient of performance (COP); and (4) the volumetric capacity $\left(Q_{\mathrm{vol}}\right)$ matching that of the baseline HFC-134a. We also considered market availability as a practical parameter influencing our decision. Table 3.5-1 shows that the selection parameters of the competing blends are very close to each other. The following four blends, based on HFC-134a, HFO-1234yf, HFO-1234ze(E) and HFC-227ea, were selected for tests in the mini-breadboard heat pump:

○ $\underline{\text { R-513A: }}$ [R-134a/1234yf $\left.\left(44 / 56^{*}\right)\right]$, GWP $=573$. R-513A was identified in our limitedscope study (blend \# 2). Its ASHRAE safety classification is A1.

○ $\underline{\mathrm{R}-450 \mathrm{~A}:}$ [R-134a/1234ze(E) $\left.\left(42 / 58^{*}\right)\right], \mathrm{GWP}=547$. R-450A was not specifically identified in the limited-scope study; however, its make-up and performance are similar to those for blend \# 9. Its ASHRAE A1 safety classification and market availability provided a practical argument for its selection.

○ Tern-1: [R-134a/1234yf/1234ze(E) (49.2/33.9/16.9*)], GWP = 640. This blend was identified in our limited-scope study (blend \#4). It was formulated using the laboratory facilities at NIST-Boulder. We adopted the Tern-1 name here for convenience.

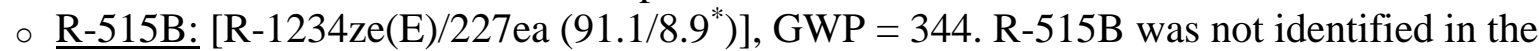
limited-scope study. We opted to include this blend because of its significantly lower GWP than those of other fluids. Considering that the preference for low-GWP fluids is expected to increase following the Montreal Protocol timetable for GWP reduction, it will be of interest to explore the performance potential of this somewhat lower-pressure blend than HFC-134a. According to our detailed cycle simulations, the COP of R-515B is within $2.6 \%$ of HFC-134a. The volumetric capacity is $27 \%$ lower, which can be mitigated by a compressor with a larger displacement.

${ }^{*}$ Composition stated in mass percent 
Table 3.5-13.5-2. Selected "best" blends from the limited-scope project (sorted by GWP) ${ }^{3}$

\begin{tabular}{|c|c|c|c|c|c|c|}
\hline & Components & $\begin{array}{l}\text { Composition } \\
\text { (molar) }\end{array}$ & GWP & $\bar{\Pi}^{*}$ & $\begin{array}{c}\mathrm{COP} / \\
\mathrm{COP}_{\mathrm{R}-134 \mathrm{a}} \\
\end{array}$ & $\begin{array}{c}Q_{\mathrm{vol}} / \\
Q_{\mathrm{vol}, \mathrm{R}-134 \mathrm{a}} \\
\end{array}$ \\
\hline & \multicolumn{6}{|c|}{ Class 1 nonflammable (predicted) } \\
\hline 1 & $\mathrm{R}-134 \mathrm{a} / 1234 \mathrm{yf}$ & $0.44 / 0.56$ & 537 & -0.1 & 0.987 & 1.025 \\
\hline 2 & $\mathrm{R}-134 \mathrm{a} / 1234 \mathrm{yf}^{* *}$ & $0.468 / 0.532$ & 573 & -0.4 & 0.988 & 1.027 \\
\hline 3 & $R-134 a / 1234 y f / 134$ & $0.48 / 0.48 / 0.04$ & 633 & -1.1 & 0.987 & 0.975 \\
\hline 4 & R-134a/1234yf/1234ze(E) $)^{\#}$ & $0.52 / 0.32 / 0.16$ & 640 & -1.2 & 0.987 & 0.989 \\
\hline 5 & $\mathrm{R}-134 \mathrm{a} / 1234 \mathrm{yf}$ & $0.52 / 0.48$ & 640 & -1.2 & 0.989 & 1.029 \\
\hline 6 & R-134a/1234yf/134 & $0.4 / 0.44 / 0.16$ & 665 & -1.3 & 0.986 & 0.958 \\
\hline 7 & $\mathrm{R}-134 \mathrm{a} / 125 / 1234 \mathrm{yf}$ & $0.44 / 0.04 / 0.52$ & 676 & -1.5 & 0.985 & 1.049 \\
\hline 8 & R-134a/227ea/1234yf & $0.40 / 0.04 / 0.56$ & 681 & -1.5 & 0.984 & 1.007 \\
\hline 9 & $\mathrm{R}-134 \mathrm{a} / 1234 \mathrm{ze}(\mathrm{E})$ & $0.60 / 0.40$ & 745 & -2.4 & 0.988 & 0.908 \\
\hline 10 & $\mathrm{R}-134 \mathrm{a} / 1234 \mathrm{yf}$ & $0.60 / 0.40$ & 745 & -2.4 & 0.990 & 1.031 \\
\hline 11 & R-134a/1234ze(E)/1243zf & $0.60 / 0.36 / 0.04$ & 750 & -1.5 & 0.990 & 0.966 \\
\hline 12 & R-134a/1234yf/1234ze(E) & $0.64 / 0.2 / 0.16$ & 799 & -3.0 & 0.990 & 0.986 \\
\hline 13 & $\mathrm{R}-134 \mathrm{a} / 152 \mathrm{a} / 1234 \mathrm{yf}$ & $0.64 / 0.04 / 0.32$ & 817 & -1.8 & 0.993 & 1.023 \\
\hline 14 & R-134a/1234yf/134 & $0.52 / 0.32 / 0.16$ & 824 & -3.2 & 0.990 & 0.966 \\
\hline 15 & $\mathrm{R}-134 \mathrm{a} / 1234 \mathrm{ze}(\mathrm{E})$ & $0.68 / 0.32$ & 852 & -3.7 & 0.991 & 0.929 \\
\hline \multirow[t]{2}{*}{16} & R-134a/1234yf/1243zf & $0.68 / 0.2 / 0.12$ & 870 & -1.1 & 0.994 & 1.020 \\
\hline & \multicolumn{6}{|c|}{ Class $2 L$ flammable (predicted) } \\
\hline 17 & $\mathrm{R}-152 \mathrm{a} / 1234 \mathrm{yf}$ & $0.08 / 0.92$ & 8 & 7.7 & 0.98 & 0.957 \\
\hline 18 & $R-134 a / 1234 y f$ & $0.20 / 0.80$ & 238 & 2.8 & 0.98 & 0.996 \\
\hline 19 & R-134a/152a/1234yf & $0.20 / 0.16 / 0.64$ & 270 & 8.7 & 0.987 & 0.984 \\
\hline 20 & R-152a/1234yf/134 & $0.16 / 0.48 / 0.36$ & 417 & 7.5 & 0.984 & 0.900 \\
\hline 21 & R-134a/1234yf & $0.36 / 0.64$ & 436 & 1.0 & 0.985 & 1.018 \\
\hline 22 & R-134a/1234yf/1243zf & $0.36 / 0.44 / 0.20$ & 451 & 5.2 & 0.988 & 1.004 \\
\hline 23 & R-134a/152a/1234yf & $0.36 / 0.20 / 0.44$ & 496 & 8.3 & 0.994 & 0.994 \\
\hline
\end{tabular}

* Normalized flammability index ${ }^{3,72}$

** Designated by ASHRAE Std. 34 as R-513A; safety classification: A1.

\# Referred to as Tern-1

\subsubsection{Selection of Three Blends for Testing in ECU}

The tests conducted in the mini-breadboard heat pump on HFC-134a and the four blends validated the prediction capability of the CYCLE_D-HX model used in the limited-scope project thus confirming the relative merits of blends established earlier. Blends R-513A, R-440A, and R-515B have the ASHRAE safety designation A1 (low toxicity, no flame propagation). While Tern-1 does not have ASHRAE classification, it can also be considered an A1 blend, since its 'non-flammability' was confirmed by the ASTM E861 test prescribed in ASHRAE Standard 34, and its three components are classified as 'low-toxicity' fluids by this standard. It is uncertain at this time whether the ASTM E861 test is stringent enough for military requirements; since this issue has not been determined yet, we decided to use the ASHRAE flammability criteria in this study. 
The following are the three blends we have selected for testing in the ECU in environmental chambers.

1. $\underline{\mathrm{R}-513 \mathrm{~A}}$ : [R-134a/1234yf $\left.(44 / 56)^{*}\right], \mathrm{GWP}=573$

$\mathrm{COP} / \mathrm{COP}_{\mathrm{R}-134 \mathrm{a}}=0.988 ; Q_{\mathrm{vol}} / Q_{\mathrm{vol}, \mathrm{R}-134 \mathrm{a}}=1.027$

This blend possesses a very good combination of three important attributes: its GWP is second to the lowest on our list; its COP is $1.2 \%$ below $\mathrm{COP}_{\mathrm{R}-134 \mathrm{a}}$, which makes it the second top COP of blends with GWP < 750; and its volumetric capacity is the highest on our list, $2.7 \%$ better than that of HFC-134a. In addition, this blend is an azeotrope and is commercially available.

2. Tern-1: [R-134a/1234yf/1234ze(E) (49.2/33.9/16.9) $)^{*}$, GWP $=640$

$\mathrm{COP} / \mathrm{COP}_{\mathrm{R}-134 \mathrm{a}}=0.987 ; Q_{\mathrm{vol}} / Q_{\mathrm{vol}, \mathrm{R}-134 \mathrm{a}}=0.989$

Compared to R-513A, Tern-1 comprises 5.2\% more of HFC-134a and includes $16.9 \%$

HFO-1234ze(E), which is less flammable (lower burning velocity) than HFO-1234yf. As a result, Turn-1 is expected to be farther away from the flammability boundary than R-513A (see Section 3.2.4) and is a more conservative choice should military criteria with respect to flammability become more rigorous (see Section 3.2.5). The simulated COP of Tern- 1 is $1.3 \%$ below $\mathrm{COP}_{\mathrm{R}-134 \mathrm{a}}$. The $1.1 \%$ lower volumetric capacity than that for $\mathrm{HFC}-134 \mathrm{a}$ is a trivial difference.

3. R-515B: [R-1234ze(E)/227ea (91.1/8.9) $)^{*}$, GWP $=344$

$\mathrm{COP} / \mathrm{COP}_{\mathrm{R}-134 \mathrm{a}}=0.973 ; Q_{\mathrm{vol}} / Q_{\mathrm{vol}, \mathrm{R}-134 \mathrm{a}}=0.738$

The main motivation to select this blend for further testing is its significantly lower GWP than those of other blends chosen. The data presented in Section 3.2.4 indicate that R515B is closer to the flammability boundary than the other blends. Per CYCLE_D-HX simulations with optimized heat exchangers, the COP of this blends is lower than $\mathrm{COP}_{\mathrm{R}-134 \mathrm{a}}$ by $2.6 \%$, and the volumetric capacity is lower than $Q_{\mathrm{vol}, \mathrm{R}-134 \mathrm{a}}$ by $26.2 \%$, which would have to be mitigated by a larger compressor and some efficiency enhancing features. Despite these shortfalls, it is of interest to explore the performance potential of this low-pressure blend in case lower-GWP fluids become strongly preferred in the future. $\mathrm{R}-515 \mathrm{~B}$ is an azeotrope and is commercially available.

By the above selection of three blends we propose to drop R-450A from further testing. The volumetric capacity of R-450A is $13.3 \%$ lower than $Q_{\mathrm{vol}, \mathrm{R}-134 \mathrm{a}}$, so it is about half the way

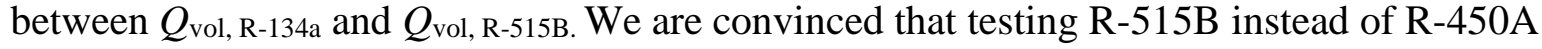
will provide more useful data (performance of a low-pressure fluid), in particular that the measurements taken on the ECU with the selected blends will provide a broad spectrum of data for validating a detail ECU simulation model. The model then can be used for predicting performance of other fluids that were not included in the ECU tests.

*Composition stated in mass percent 


\section{Conclusions to Date}

Task 1. We have carried out measurements on the thermophysical properties of refrigerant blends identified in the limited-scope project. For three blends (at two compositions each) we have completed comprehensive measurements comprising vapor-liquid equilibria (VLE), density $(P, \rho, T, x)$, speed of sound, and thermal conductivity; these measurements covered a combined temperature range of 230 to $400 \mathrm{~K}$, with pressures up to $50 \mathrm{MPa}$. For three additional blends (also at two compositions each) we have carried out only VLE measurements. The measurements were selected to provide an optimal data set for the purposes of fitting mixture property models, and these data have allowed us to improve the refrigerant mixture models needed for conducting the MBHP tests (Task 3), refrigerant twophase heat-transfer tests (Task 4), and ECU tests (Task 7). While the improved property models will be important for these tasks, no major deficiencies were identified in the models used in the limited-scope project; in other words, the selection of "best blends" made in the limited-scope project remain valid. In the second phase of the project these data, along with literature data, will be used to develop a mixture model optimized for blends of low-GWP fluids, especially blends containing HFOs; this optimized model will be used in the detailed simulations of Task 7.

Task 2.

Specific interim conclusions are:

1. Three blends Tern-1, R-513A, and R-450A are of similar flammability, with R-450A slightly better. While R-515B is non-flammable by the E681 test, it is close to the border of flammability. If a Class 1 flammability rating via ASHRAE Standard 34 (i.e., based on the ASTM E681 test) is acceptable, then R-515B may be the best refrigerant, since it has lowest GWP. But this depends upon how the live-fire test compares to the E681 test.

2. The calculated overall reaction rate predicts that for the candidate blends, the effect of humidity in the air will be small for an increase from 0 to $50 \%$ r.h., but large for $50 \%$ r.h. to $100 \%$ r.h. Thus, levels of humidity above 0.014 moles $\mathrm{H}_{2} \mathrm{O} /$ mole air (50 \% r.h. at $23{ }^{\circ} \mathrm{C}$ ) may have large effects on the flammability of the blends.

3. HFC-134a should be tested at high ambient temperature and high humidity as a benchmark.

4. We suggest live-fire tests of HFC-134a with increasing amounts of added HFO-1234yf to enable correlation to the small-scale experimental and numerical results.

Task 3. The Mini-Breadboard Heat Pump (MBHP) was used to experimentally evaluate HFC-134a and four candidate low-GWP blends: R-513A, R-450A, R-515B, and Tern-1. The purpose of these tests was to: (1) validate the CYCLE_D-HX simulation model ${ }^{52,53}$ used in the limited-scope project ${ }^{1}$, and (2) qualify the three 'best' blends for testing in a military ECU (Task 7). Cycle performance of each fluid was measured over a range of capacity including $(1.3,1.5$, and 1.7$) \mathrm{kW}$, where $1.5 \mathrm{~kW}$ was the rating point. The varying capacity provided measurements to verify the model's prediction ability over a range of mass and heat flux. Each capacity point was tested both without and with a LLSL-HX. The test-to-test variation, largely driven by compressor efficiency, yielded representative average COP and

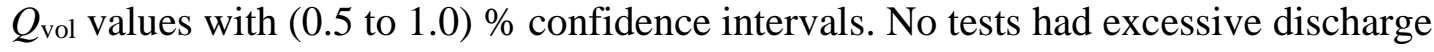
temperatures or other hardware-related problems. 
All 121 experimental tests were then simulated using CYCLE_D-HX. For R-513A, R-450A, and R-515B in the basic cycle (tests without the LLSL-HX), the model-predicted values were within the confidence intervals of the experimental results, within about $\pm 1.5 \%$ of the curve fit. For Tern-1, the model overpredicted the experimental data by about $3 \%$. For the tests with the LLSL-HX, at $1.5 \mathrm{~kW}$, the COP and $Q_{\text {vol }}$ were overpredicted by (0 to 3$) \%$. Importantly, the model provided the same relative COP and $Q_{\text {vol }}$ ranking as the experimental data, giving confidence to the screening study performed in the limited-scope project.

Task 4. An experimental apparatus was used to establish and measure 432 convective-boiling heat-transfer coefficients for R-515B, R-450A, R-513A, and HFC-134a in a micro-fin tube. The heat-transfer coefficients were local (a function of thermodynamic quality) and were obtained with a fluid-heating boundary condition. It has been shown that data taken with an electrically-heated boundary condition can differ significantly in its behavior with respect to quality as compared to the fluid-heating boundary condition. These measurements were used to develop an improved correlation for the local Nusselt number for the micro-fin tube. The new correlation predicted $71 \%$ of the measured convective boiling Nusselt numbers for R515B, R-450A, R-513A, and HFC-134a to within $\pm 20 \%$. The correlation is valid for Reynolds numbers (Re) between 1000 and 14000, Boiling numbers (Bo) between 0.000002 and 0.001, and Bond numbers $\left(\mathrm{B}_{\mathrm{nd}}\right)$ between 0.002 and 0.05. The correlation was developed with data where the refrigerant reduced temperature ranged between approximately 0.71 and 0.94. The data taken and the new correlation was tailored for use in Task 7 of the project for simulations of the ECU system with optimized heat exchangers.

Task 5. We selected R-513A [R-134a/1234yf (44/56) $\left.{ }^{*}\right]$, Tern-1 [R-134a/1234yf/1234ze(E) $\left.(49.2 / 33.9 / 16.9)^{*}\right]$, and R-515B [R-1234ze(E)/227ea (91.1/8.9)*] for ECU testing in the NIST environmental chambers. Each of these blends has the potential of being a fluid of choice

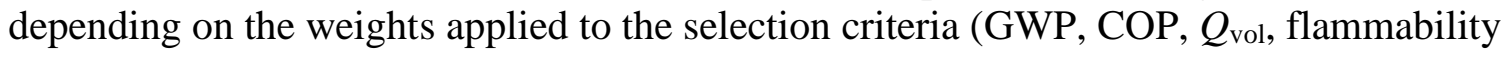
characteristics, see Section 3.5.3).

In addition, the ECU measurements with these fluids will provide a diverse database for validating a detailed ECU system model, which can be used later to simulate performance of other blends of possible interest.

In the above blend selection, we adopted the ASTM E681 test method as stipulated by ASHRAE Standard 34 for qualifying 'non-flammability' of refrigerants. Based on the present interim results, it will likely be possible to replace HFC-134a with a blend having at least $50 \%$ lower GWP than that of HFC-134a. If military requirements for 'non-flammability' are more stringent than the E681 standard, a smaller reduction of GWP will be possible with qualifying blends.

Future Tasks. The remaining work (Task 7) includes experimental and simulation components. In the experimental effort, NIST will procure, install, and instrument a 3-ton military ECU in the NIST environmental chambers and will conduct performance tests according to the AHRI Standard 210/240 ${ }^{54}$ using HFC-134a, R-513A, Tern-1, and R-515B. The tests will be conducted at three standard test conditions consisting of the indoor $26.7^{\circ} \mathrm{C}$

* Compositions stated as mass percent 
dry bulb and $15.8{ }^{\circ} \mathrm{C}$ dew point and three outdoor temperatures of $27.8{ }^{\circ} \mathrm{C}, 35.0{ }^{\circ} \mathrm{C}$, and $46.1^{\circ} \mathrm{C}$. These tests will include "soft optimization" of the ECU, which will entail a modification of the expansion device and optimization of the refrigerant charge but the same heat exchangers and compressor will be used for all fluids.

Within the simulation effort, NIST will apply two NIST 'first-principles-based' simulation models: EVAP-COND ${ }^{73}$ and ACSIM. EVAP-COND is a software package for predicting performance of finned-tube evaporators and condensers. It is equipped with a computationalintelligence-based module to optimize the refrigerant circuitry. ACSIM is an in-house system model, which utilizes EVAP-COND components for simulating evaporators and condensers.

NIST will update ACSIM and EVAP-COND with improved blend thermophysical properties routines (from Task 1) and two-phase heat-transfer routines (from Task 4), and will 'tune' the EVAP-COND and ACSIM models to predict ECU laboratory results. The EVAPCOND's optimization module will be used to arrive at the optimum refrigerant circuitry for each studied blend. These optimized refrigerant circuit architectures will be used by ACSIM to predict the performance potential of competing blends in completely optimized ECU systems. The analysis will include the performance of carbon dioxide as a refrigerant.

The preparation of the final report will conclude this project. The report will document research results from all tasks. In the final part, it will provide a complete performance characterization of HFC-134a and three selected low-GWP blends in a soft-optimized ECU and in an ECU with optimized heat exchangers, based on detailed ECU simulations. 


\section{Literature Cited}

1. Domanski, P. A.; McLinden, M. O.; Bell, I. H.; Linteris, G. T. Low-GWP Alternative Refrigerant Blends for HFC-134a; NIST Technical Note 2014; National Institute of Standards and Technology: Gaithersburg, MD, 2018.

2. McLinden, M. O.; Brown, J. S.; Brignoli, R.; Kazakov, A. F.; Domanski, P. A., Limited options for low-global-warming-potential refrigerants. Nat Commun 2017, 8 .

3. Bell, I. H.; Domanski, P. A.; McLinden, M. O.; Linteris, G. T., The hunt for nonflammable refrigerant blends to replace R-134a. Int J Refrig 2019, 104, 484-495.

4. Wagner, W.; Kleinrahm, R., Densimeters for very accurate density measurements of fluids over large ranges of temperature, pressure, and density. Metrologia 2004, 41, S24-S39.

5. McLinden, M. O.; Lösch-Will, C., Apparatus for wide-ranging, high-accuracy fluid (p, $\rho$, T) measurements based on a compact two-sinker densimeter. J. Chem. Thermodyn. 2007, $39,507-530$.

6. Healy, J.; DeGroot, J. J.; Kestin, J., The theory of the transient hot-wire method for measuring the thermal conductivity. Physica 1976, C82, 392-408.

7. Bell, I. H.; Lemmon, E. W., Automatic Fitting of Binary Interaction Parameters for Multifluid Helmholtz-Energy-Explicit Mixture Models. J. Chem. Eng. Data 2016, 61 (11), 3752-3760-3752-3760.

8. Kunz, O.; Wagner, W., The GERG-2008 wide-range equation of state for natural gases and other mixtures: An expansion of GERG-2004. J. Chem. Engr. Data 2012, 57, 30323091.

9. Lemmon, E. W.; Jacobsen, R. T., Equations of state for mixtures of R-32, R-125, R-134a, R-143a, and R-152a. Journal of Physical and Chemical Reference Data 2004, 33, 593620.

10. Williams, F. A., A unified view of fire suppression. J. Fire Flamma. 1974, 5, 54-63.

11. Davis, S. G.; Pagliaro, J. L.; Debold, T. F.; van Wingerden, M.; van Wingerden, K., Flammability and explosion characteristics of mildly flammable refrigerants. Journal of Loss Prevention in the Process Industries 2017, 49, 662-674.

12. ASHRAE, ANSI/ASHRAE Standard 34-2019, Designation and Safety Classification of Refrigerants. American Society of Heating, Refrigerating and Air-Conditioning Engineers: Atlanta, GA, 2019.

13. Papas, P.; Verma, P.; Lord, R.; Burns, L., Turbulent deflagrations of mildly flammable refrigerant-air mixtures. Journal of Loss Prevention in the Process Industries 2019, 61, 255-261.

14. Linteris, G.; Bell, I.; McLinden, M., An Empirical Model for Refrigerant Flammability Based on Molecular Structure and Thermodynamics. International Journal of Refrigeration 2019, 104, 144-150.

15. Linteris, G. T.; Babushok, V. I.; Pagliaro, J. L.; Burgess, J. D. R.; Manion, J. A.; Takahashi, F.; Katta, V. R.; Baker, P. T., Understanding overpressure in the FAA aerosol can test by $\mathrm{C}_{3} \mathrm{H}_{2} \mathrm{~F}_{3} \mathrm{Br}$ (2-BTP). Combust. Flame 2016, 167, 452-462.

16. Linteris, G.; Pagliaro, J. L.; Sunderland, P. B. Test Results Prepared for Honeywell: Igniter Material Effects in the Japanese High Pressure Gas Law Test; NIST Technical Note 1902; National Institute of Standards and Technology: Gaithersburg, MD, 2016; p 52.

17. ASHRAE ANSI/ASHRAE Standard 34-2019, Designation and Safety Classification of Refrigerants; American Society of Heating,, Refrigerating and Air-Conditioning Engineers,: Atlanta, GA, 2019.

18. ISO International Organization for Standarization (ISO-817): Refrigerants-Designation and safety classification. ; Geneva, Switzerland, 2017.

19. ASTM, ASTM E681-09: Standard test method for concentration limits of flammability of chemicals (vapors and gases). In ASTM Fire Standards, Sixth ed.; American Society of Testing and Materials: West Conshohocken, PA, 2015. 
20. Richard, R. G., Personal Communication. 2011.

21. Richard, R. G.; Kannoy, D.; Kusmierz, A. Binary Refrigerant Flame Boundary Concentrations (ASHRAE Research Project 1507-RP Final Report); ASHRAE,: Atlanta, GA, 2017; p 59.

22. Kim, D. K.; Klieger, A. E.; Lomax, P. Q.; McCoy, C. G.; Reymann, J. Y.; Sunderland, P. B., An improved test method for refrigerant flammability limits in a 12 L vessel. Sci. Technol. Built Environ. 2018, 24 (8), 861-866.

23. Richard, R. G. Refrigerant flammability testing in large volume vessels; DOE/CE/2381087; Allied Signal Buffalo Research Laboratories: Buffalo, NY, March 1998, 1998; p 11 p.

24. Kelley, A. P.; Jomaas, G.; Law, C. K., Critical radius for sustained propagation of sparkignited spherical flames. Combust. Flame 2009, 156 (5), 1006-1013.

25. Berger, L.; Hesse, R.; Kleinheinz, K.; Hegetschweiler, M. J.; Attili, A.; Beeckmann, J.; Linteris, G. T.; Pitsch, H., A DNS study of the impact of gravity on spherically expanding laminar premixed flames. Combust. Flame 2020, 216, 412-425.

26. Pagliaro, J. L. Inhibition of laminar premixed flames by Halon 1301 alternatives; Ph.D. Thesis; University of Maryland: College Park, MD, 2015, 2015.

27. Pagliaro, J. L.; Linteris, G. T.; Sunderland, P. B.; Baker, P. T., Combustion inhibition and enhancement of premixed methane-air flames by halon replacements. Combust. Flame 2015, 162 (1), 41-49.

28. Linteris, G. T.; Pagliaro, J. L. Burning Velocity Measurements and Simulations for Understanding the Performance of Fire Suppressants in Aircraft; NIST TN 1904; National Institute or Standards and Technology: Gaithersburg, MD, 2016, 2016; pp 1-49.

29. Linteris, G. T.; Pagliaro, J. L. Test results prepared for Honeywell: Flammability of refrigerants in the Japanese high pressure gas law test; NISTIR 7178; Gaithersburg MD, 10/05/2011, 2011; p 48 p.

30. Chen, Z.; Burke, M. P.; Ju, Y., On the critical flame radius and minimum ignition energy for spherical flame initiation. Proc. Combust. Inst. 2011, 33 (1), 1219-1226.

31. Goodwin, D. G.; Moffat, H. K.; Speth, R. L. Cantera: An object-oriented software toolkit for chemical kinetics, thermodynamics, and transport processes. http:/www.cantera.org, Version 2.1.1; California Institute of Technology: Pasedena, CA, 2016.

32. Peters, N., Turbulent Combustion. Cambridge University Press: Cambridge, 2000.

33. Hansen, O. R.; Hinze, P.; Engel, D.; Davis, S., Using computational fluid dynamics (CFD) for blast wave predictions. Journal of Loss Prevention in the Process Industries 2010, 23 (6), 885-906.

34. Hisken, H.; Enstad, G.; Middha, P.; van Wingerden, K., Investigation of concentration effects on the flame acceleration in vented channels. Journal of Loss Prevention in the Process Industries 2015, 36, 447-459.

35. Linteris, G. T.; Burgess, D. R.; Babushok, V.; Zachariah, M.; Tsang, W.; Westmoreland, P., Inhibition of premixed methane-air flames by fluoroethanes and fluoropropanes. Combust. Flame 1998, 113 (1-2), 164-180.

36. Linteris, G. T.; Truett, L., Inhibition of premixed methane-air flames by fluoromethanes. Combust. Flame 1996, 105 (1-2), 15-27.

37. Katta, V. R.; Takahashi, F.; Linteris, G. T., Fire-suppression characteristics of $\mathrm{CF}_{3} \mathrm{H}$ in a cup burner. Combust. Flame 2006, 144 (4), 645-661.

38. Babushok, V. I.; Linteris, G. T.; Meier, O., Combustion properties of halogenated fire suppressants. Combust Flame 2012, 159, 3569-3575.

39. Linteris, G. T.; Burgess, D. R.; Takahashi, F.; Katta, V. R.; Chelliah, H. K.; Meier, O., Stirred reactor calculations to understand unwanted combustion enhancement by potential halon replacements. Combust. Flame 2012, 159 (3), 1016-1025.

40. Pagliaro, J. L.; Bouvet, N.; Linteris, G. T., Premixed flame inhibition by $\mathrm{CF}_{3} \mathrm{Br}$ and $\mathrm{C}_{3} \mathrm{H}_{2} \mathrm{~F}_{3} \mathrm{Br}$ (2-BTP). Combust. Flame 2016, 169, 272-286. 
41. Takahashi, F.; Katta, V. R.; Linteris, G. T.; Babushok, V. I., Combustion inhibition and enhancement of cup-burner flames by $\mathrm{CF}_{3} \mathrm{Br}, \mathrm{C}_{2} \mathrm{HF}_{5}, \mathrm{C}_{2} \mathrm{HF}_{3} \mathrm{Cl}_{2}$, and $\mathrm{C}_{3} \mathrm{H}_{2} \mathrm{~F}_{3} \mathrm{Br}$. Proc. Combust. Inst. 2015, 35, 2741-2748.

42. Takahashi, F.; Katta, V. R.; Linteris, G. T.; Meier, O. C., Cup-burner flame structure and extinguishment by $\mathrm{CF} 3 \mathrm{Br}$ and $\mathrm{C} 2 \mathrm{HF} 5$ in microgravity. Proc. Combust. Inst. 2013, 34, 2707-2717.

43. Linteris, G. T.; Babushok, V. I.; Sunderland, P. B.; Takahashi, F.; Katta, V. R.; Meier, O., Unwanted combustion enhancement by $\mathrm{C} 6 \mathrm{~F} 12 \mathrm{O}$ fire suppressant. Proc. Combust. Inst. 2013, 34, 2683-2690.

44. Burgess Jr, D. R.; Zachariah, M. R.; Tsang, W.; Westmoreland, P. R. Thermochemical and Chemical Kinetic Data for Fluorinated Hydrocarbons; NIST Technical Note 1412; National Institute of Standards and Technology: Gaithersburg, MD, 1995.

45. Burgess, D. R.; Babushok, V. I.; Linteris, G. T.; Manion, J. A., A Chemical Kinetic Mechanism for 2-Bromo-3,3,3-trifluoropropene (2-BTP) Flame Inhibition. Int. J. Chem. Kinet. 2015, 47 (9), 533-563.

46. Babushok, V. I.; Burgess, D. R.; Kim, D. K.; Hegetschweiler, M. J.; Linteris, G. T. Kinetic Model for Refrigerant-Air Flames; NIST Technical Note xxxx; National Institute of Standards and Technology: Gaithersburg, MD, 2021, 2021; $p$ to be submitted.

47. Linteris, G. T.; Babushok, V. I., Laminar burning velocity predictions for $\mathrm{C}_{1}$ and $\mathrm{C}_{2}$ hydrofluorocarbon refrigerants with air. J Fluorine Chem 2020, 230.

48. Babushok, V. I.; Burgess, D. R.; Hegetschweiler, M. J.; Linteris, G. T., Flame propagation in the mixtures of $\mathrm{O} 2 / \mathrm{N} 2$ oxidizer with fluorinated propene refrigerants (CH2CFCF3, CHFCHCF3, CH2CHCF3). Combust. Sci. Technol. 2020, 1-24.

49. Hegetschweiler, M. J.; Linteris, G. T. Data Reduction Tool for Spherical Constant Volume Flame Experiments; NIST Technical Note in preparation; National Institute of Standards and Technology: Gaithersburg, MD, 2021; p 999.

50. Kim, D. K.; Babushok, V. I.; Burgess, D. R.; Hegetschweiler, M. J.; Linteris, G. T., Burning Velocity of Blends of R-152a with R-134a or R-1234yf and air. Combust. Sci. Technol. 2020, to be submitted.

51. Barat, R. B., Correlating laminar burning velocities using perfectly stirred reactor theory. Chem. Eng. Sci. 2001, 56, 2761-2766.

52. Brown, J. S., Brignoli, R., Domanski, P.A., Yoon, Y.J. CYCLE_D-HX: NIST Vapor Compression Cycle Model Accounting for Refrigerant Thermodynamic and Transport Properties; Version 2.0, National Institute of Standards and Technology: Gaithersburg, MD, 2021.

53. Brignoli, R.; Brown, J. S.; Skye, H. M.; Domanski, P. A., Refrigerant performance evaluation including effects of transport properties and optimized heat exchangers. Int $J$ Refrig 2017, 80, 52-65.

54. AHRI, 210/240-2017 Standard for Performance Rating of Unitary A/C and Air Source Heat Pump Equipment. Arlington, VA, 2017.

55. Skye, H. M. Heat Pump Test Apparatus for the Evaluation of Low Global Warming Potential Refrigerants; NIST Technical Note 1895, National Institute of Standards and Technology: Gaithersburg, MD, 2015.

56. McLinden, M. O.; Radermacher, R., Methods for comparing the performance of pure and mixed refrigerants in the vapour compression cycle. International Journal of Refrigeration 1987, 10 (6), 318-325.

57. Kedzierski, M. A.; Park, K. J., Horizontal Convective Boiling of R134a, R1234yf/R134a, and R1234ze(E) within a Micro-Fin Tube. J Enhanc Heat Transf 2013, 20 (4), 333-346.

58. Kattan, N.; Thome, J. R.; Favrat, D., R-502 and two near-azeotropic alternatives. Part 1: In-tube flow-boiling tests. ASHRAE Transactions 1995, 101 (1), 491-508.

59. Hamilton, L.; Kedzierski, M.; Kaul, M., Horizontal convective boiling of pure and mixed refrigerants within a micro-fin tube. J Enhanc Heat Transf 2008, 15 (3).

60. Kedzierski, M. A.; Kang, D., Horizontal convective boiling of R1234yf, R134a, and R450A within a micro-fin tube. Int J Refrig 2018, 88, 538-551. 
61. Kedzierski, M. A.; Lin, L.; Kang, D., Pool Boiling of Low-Global Warming Potential Replacements for R134a on a Reentrant Cavity Surface. J Heat Trans-T Asme 2018, 140 (12).

62. Solomon, S.; Burkholder, J. B.; Ravishankara, A. R.; Garcia, R. R., Ozone depletion and global warming potentials of $\mathrm{CF}_{3}$ I. J. Geo. Res. Atmospheres 1994, 99, 20929-20935.

63. Bell, I. H.; McLinden, M. O., The status of thermodynamic data and models for $\mathrm{CF}_{3} \mathrm{I}$ and its mixtures. Int. J. Thermopysics 2020, 41, 134.

64. Yang, Z.; Liu, H.; Wu, X., Theoretical and experimental study of the inhibition and inert effect of HFC125, HFC227ea and HFC13I1 on the flammability of HFC32. Process Safety and Environmental Protection 2012, 90, 311-316.

65. Yana Motta, S. F. Submission to ASHRAE SSPC 34 Committee. Request for Refrigerant Designation and Safety Classification For $R-32 / R-125 / C F_{3} I$ (49.0/11.5/39.5 \% by mass) Zeotropic Refrigerant Blend; Honeywell International, Inc., Buffalo, NY: 2018.

66. ASHRAE, Addendum t to Standard 34-2019, Designation and Safety Classification of Refrigerants. American Society of Heating, Refrigerating and Air-Conditioning Engineers: 2019.

67. National Research Council, Iodotrifluoromethane: Toxicity Review (2004). Washington, DC: The National Academies Press.

68. Allgood, C.; Johnston, P.; Kim, S.; Kujak, S.; Yana Motta, S., Roundtable: A conversation on refrigerants. ASHRAE J. 2021, March 2021, 30-37.

69. Akasaka, R.; Fukuda, S.; Sakoda, N.; Higashi, Y. In pvT Property measurement and development of an equation of state for new refrigerant HFO1132(E), Proceedings of the 2020 JSRAE Annual Conference, Tsu-city, September 8-11, 2020; Tsu-city, 2020.

70. Perera, U. A.; Miyazaki, T.; Sakoda, N.; Higashi, Y. In Determination of saturation pressure and critical pressure for new refrigerant HFO1132(E), Proceedings of the 2020 JSRAE Annual Conference, Tsu-city, September 8-11, 2020; Tsu-city, 2020.

71. McLinden, M. O.; Brown, J. S.; Brignoli, R.; Kazakov, A. F.; Domanski, P. A., Limited options for low-global-warming-potential refrigerants. Nat. Comm. 2017, 8, 14476.

72. Linteris, G. T.; Bell, I. H.; McLinden, M. O., An empirical model for refrigerant flammability based on molecular structure and thermodynamics. International Journal of Refrigeration 2019, 104, 144-150.

73. Domanski, P. A.; Yashar, D. A.; Wojtusiak, J. EVAP-COND, Version 5.0; Simulation Models for Finned-Tube Heat Exchangers with Circuitry Optimization, National Institute of Standards and Technology, Gaithersburg, MD: 2021.

74. Outcalt, S. L.; Lemmon, E. W., Bubble point measurements of eight binary mixtures for organic rankine cycle applications. J. Chem. Engr. Data 2013, 58, 1853-1860.

75. Hust, J. G.; Filla, J.; Smith, D. R., A modified digital PID temperature controller for thermal property measurements. Journal of Thermal Insulation 1987, 11 (10), 102-107.

76. McLinden, M. O.; Kleinrahm, R.; Wagner, W., Force transmission errors in magnetic suspension densimeters. Int. J. Thermophys. 2007, 28, 429-448.

77. McLinden, M. O.; Richter, M., Application of a two-sinker densimeter for phaseequilibrium measurements: A new technique for the detection of dew points and measurements on the (methane + propane) System. J. Chem. Thermodyn. 2016, 99, 105115.

78. McLinden, M. O.; Splett, J. D., A liquid density standard over wide ranges of temperature and pressure based on toluene. J. Res. Natl. Inst. Stand. Technol. 2008, 113, 29-67.

79. McLinden, M. O., Thermodynamic properties of propane. I. p- $\rho$-T behavior from $265 \mathrm{~K}$ to $500 \mathrm{~K}$ with pressures to $36 \mathrm{MPa}$. J. Chem. Eng. Data 2009, 54, 3181-3191.

80. Lemmon, E. W.; McLinden, M. O.; Wagner, W., Thermodynamic properties of propane. III. A Reference Equation of State for Temperatures from the Melting Line to $650 \mathrm{~K}$ and Pressures up to $1000 \mathrm{MPa}$. Journal of Chemical and Engineering Data 2009, 54, 31413180. 
81. Meier, K.; Kabelac, S., Thermodynamic Properties of Propane. IV. Speed of Sound in the Liquid and Supercritical Regions. J. Chem. Engr. Data 2012, 57, 3391-3398.

82. Roder, H. M., A transient hot wire thermal conductivity apparatus for fluids. J Res Natl Bur Stand 1981, 86 (5), 457-493.

83. Healy, J. J.; de Groot, J. J.; Kestin, J., The theory of the transient hot-wire method for measuring thermal conductivity. Physica 1976, 82, 392-408.

84. Harris, G. L.; Torres, J. A. Selected laboratory and measurement practices and procedures, to support basic mass calibrations; NISTIR 6969; National Institute of Standards and Technology: 2003.

85. Richter, M.; McLinden, M. O., Vapor-phase (p, $\rho, T, x)$ behavior and virial coefficients for the (methane + propane) system. J. Chem. Eng. Data 2014, 59, 4151-4164.

86. Rosenfeld, Y., Relation between the transport coefficients and the internal entropy of simple systems. Phys. Rev. A 1977, 15, 2545-2549-2545-2549.

87. Bell, I. H.; Messerly, R.; Thol, M.; Costigliola, L.; Dyre, J., Modified Entropy Scaling of the Transport Properties of the Lennard-Jones Fluid. J. Phys. Chem. B 2019, 123 (29), 6345-6363-6345-6363.

88. Bell, I. H., Entropy Scaling of Viscosity - I: A Case Study of Propane. J. Chem. Eng. Data 2020, 3203-3215-3203-3215.

89. Bell, I. H., Entropy Scaling of Viscosity-II: Predictive Scheme for Normal Alkanes. J. Chem. Eng. Data 2020, 65 (11), 5606-5616-5606-5616.

90. Yang, X.; Xiao, X.; May, E. F.; Bell, I. H., Entropy Scaling of Viscosity-III: Application to Refrigerants and Their Mixtures. J. Chem. Eng. Data 2021.

91. Liu, H.; Yang, F.; Yang, Z.; Duan, Y., Modeling the viscosity of hydrofluorocarbons, hydrofluoroolefins and their binary mixtures using residual entropy scaling and cubicplus-association equation of state. J. Mol. Liq. 2020, 308, 113027-113027.

92. Lötgering-Lin, O.; Gross, J., Group Contribution Method for Viscosities Based on Entropy Scaling Using the Perturbed-Chain Polar Statistical Associating Fluid Theory. Ind. Eng. Chem. Res. 2015, 54 (32), 7942-7952-7942-7952.

93. Lötgering-Lin, O.; Fischer, M.; Hopp, M.; Gross, J., Pure Substance and Mixture Viscosities Based on Entropy Scaling and an Analytic Equation of State. Ind. Eng. Chem. Res. 2018, 57 (11), 4095-4114-4095-4114.

94. Fouad, W. A.; Alasiri, H., Molecular dynamic simulation and SAFT modeling of the viscosity and self-diffusion coefficient of low global warming potential refrigerants. $J$. Mol. Liq. 2020, 317, 113998-113998.

95. Bell, I. H.; Dyre, J. C.; Ingebrigtsen, T. S., Excess-entropy scaling in supercooled binary mixtures. Nature Communications 2020, 11 (1).

96. Kondo, S.; Takizawa, K.; Takahashi, A.; Tokuhashi, K.; Sekiya, A., Flammability limits of five selected compounds each mixed with HFC-125. Fire Saf. J. 2009, 44 (2), 192-197.

97. Shebeko, Y. N.; Azatyan, V. V.; Bolodian, I. A.; Navzenya, V. Y.; Kopyov, S. N.; Shebeko, D. Y.; Zamishevski, E. D., The influence of fluorinated hydrocarbons on the combustion of gaseous mixtures in a closed vessel. Combust. Flame 2000, 121 (3), 542547.

98. Pagliaro, J. L.; Linteris, G. T.; Babushok, V. I., Premixed flame inhibition by $\mathrm{C}_{2} \mathrm{HF}_{3} \mathrm{Cl}_{2}$ and $\mathrm{C}_{2} \mathrm{HF}_{5}$. Combust. Flame 2016, 163, 54-65.

99. Shephard, J. E.; Krok, J. C.; Lee, J. J. Spark Ignition Energy Measurements in Jet A; Calif. Inst. Tech: 1999.

100. Metghalchi, M.; Keck, J. C., Laminar burning velocity of propane-air mixtures at high temperature and pressure. Combust. Flame 1980, 38, 143-154.

101. Metghalchi, M.; Keck, J. C., Burning velocities of mixtures of air with methanol, isooctane, and indolene at high pressure and temperature. Combust. Flame 1982, 48, 191210. 
102. Hill, P. G.; Hung, J., Laminar Burning Velocities of Stoichiometric Mixtures of Methane with Propane and Ethane Additives. Combustion Science and Technology 1988, 60, 7-30.

103. Takizawa, K.; Takahashi, A.; Tokuhashi, K.; Kondo, S.; Sekiya, A., Burning velocity measurement of fluorinated compounds by the spherical-vessel method. Combust Flame 2005, 141 (3), 298-307.

104. Lewis, B.; von Elbe, G., Combustion, Flames, and Explosions of Gases. 1961.

105. Saeed, K.; Stone, C. R., Measurements of the laminar burning velocity for mixtures of methanol and air from a constant-volume vessel using a multizone model. Combustion and Flame 2004, 139 (1-2), 152-166.

106. Eisazadeh-Far, K.; Moghaddas, A.; Al-Mulki, J.; Metghalchi, H., Laminar burning speeds of ethanol/air/diluent mixtures. Proc. Combust. Inst. 2011, 33 (1), 1021-1027.

107. Williams, F. A. In A review of some theoretical considerations of turbulent flame structure, AGARD Conference Proceeding, 1975.

108. Takizawa, K.; Takahashi, A.; Tokuhashi, K.; Kondo, S.; Sekiya, A., Burning velocity measurement of HFC-41, HFC-152a, and HFC-161 by the spherical-vessel method. $J$ Fluorine Chem 2006, 127 (12), 1547-1553.

109. Marshall, S. P.; Taylor, S.; Stone, C. R.; Davies, T. J.; Cracknell, R. F., Laminar burning velocity measurements of liquid fuels at elevated pressures and temperatures with combustion residuals. Combustion and Flame 2011, 158 (10), 1920-1932.

110. Babushok, V. I.; Burgess Jr, D. R.; Kim, D. K.; Hegetschweiler, M. J.; Linteris, G. T. Modeling of Combustion of Fluorine-Containing Refrigerants; NIST Technical Note in preparation; National Institute of Standards and Technology: Gaithersburg, MD, 2021; p 999. 


\section{Appendix A: Details of Experiments and Modeling}

\section{A.1. Task 1 - Details of Thermophysical Properties Measurements and Modeling}

A.1.1. Vapor-Liquid Equilibria

A schematic of the instrument used to make the measurements is shown in Figure A.1-1; it is an updated version of the instrument described by Outcalt and Lemmon. ${ }^{74}$ The heart of the instrument was a cylindrical stainless steel measuring cell with a sapphire window on each end so that the liquid level in the cell was visible. The cell had an internal volume of approximately $30 \mathrm{~mL}$. The operating range of the apparatus was $270 \mathrm{~K}$ to $360 \mathrm{~K}$, with pressures to $7 \mathrm{MPa}$.

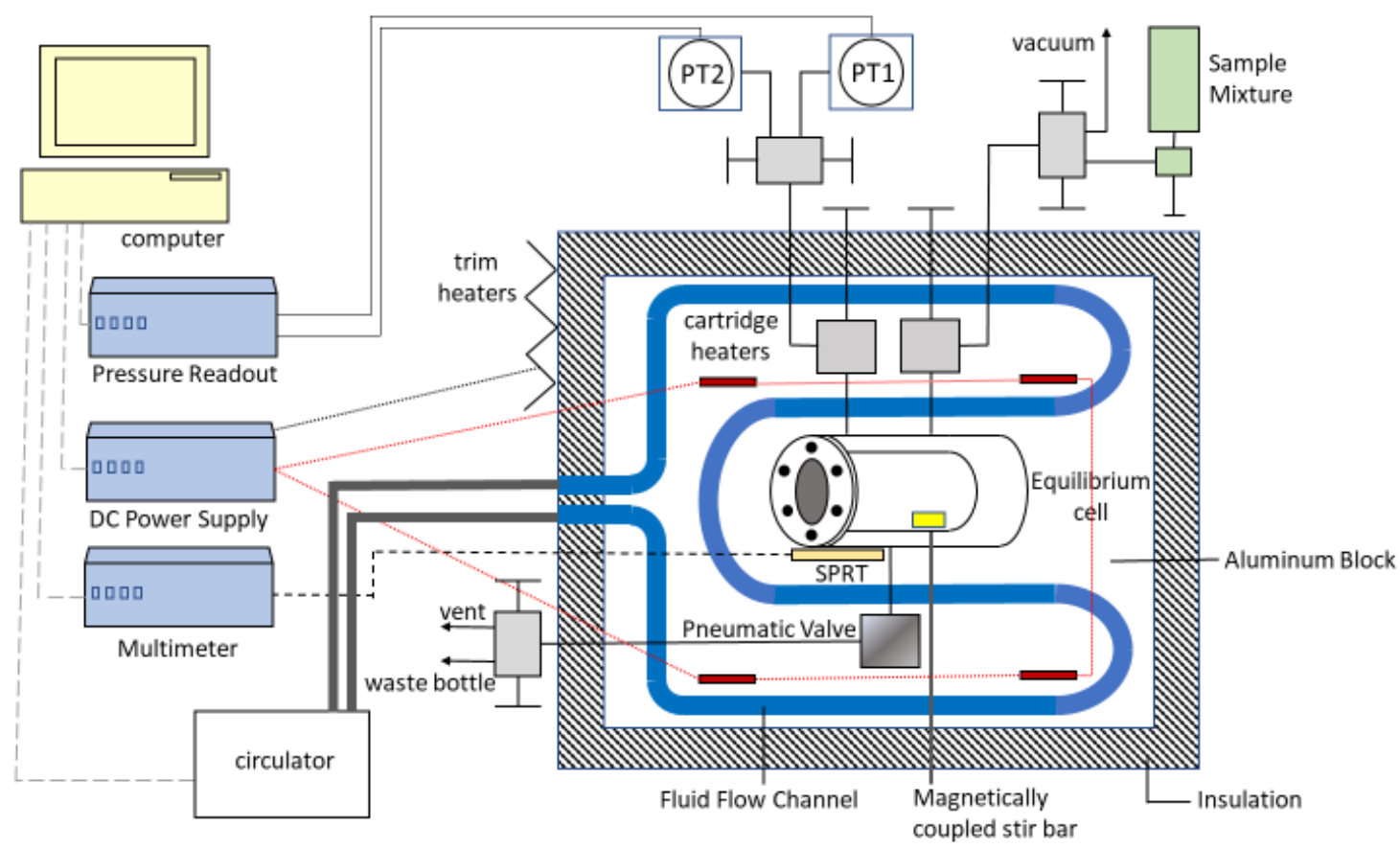

Figure A.1-1. Schematic of instrument for vapor liquid equilibria measurements.

Main components of the instrument include a stainless steel equilibrium cell housed in a thermostated aluminum block, standard platinum resistance thermometer (SPRT), and two oscillating quartz crystal pressure transducers (PT1 and PT2) with maximum pressure ranges of $0.7 \mathrm{MPa}$ and $7 \mathrm{MPa}$.

The cell was fitted inside a temperature-controlled, insulated aluminum block. The valves for filling the system were installed in the aluminum block to limit the sample volume outside the temperature-controlled zone. Cooling fluid from an external refrigerated circulator flowed through channels drilled into the aluminum block. Cartridge heaters installed in the aluminum block provided heat for operation above ambient temperature. Thin-film type heaters were adhered to the outside of the block for fine control of the temperature. Temperature control was automated with the modified PID algorithm of Hust et al. ${ }^{75}$ 
Temperature was measured with a standard platinum resistance thermometer (SPRT) installed in a thermowell adjacent to the measuring cell. The pressure was measured with one of two oscillating-quartz-crystal pressure transducers connected to the system through one of the ports at the top of the cell. These transducers had full-scale ranges of $0.7 \mathrm{MPa}$ and 7.0 MPa; the low-range transducer had the higher sensitivity, and it was used whenever possible. The transducers were thermostatted in separate thermostats maintained at $313 \mathrm{~K}$.

For loading, the sample bottle was connected to the system in an inverted position so that the liquid phase was loaded into the cell. Prior to loading a sample into the system, the system was evacuated and cooled to approximately $270 \mathrm{~K}$, and the pressure reading under vacuum was recorded. The sample was then quickly loaded into the system until only a small vapor space remained in the equilibrium cell. Stirring of the sample was effected by a stir bar inside the cell that was magnetically coupled to an external motor.

By loading the sample from the liquid phase of the sample bottle and filling the measuring cell nearly full, the liquid-phase composition in the cell was nearly that of the bulk composition of the sample bottle. Small corrections to the composition were made using the mixture model in REFPROP and assuming equilibrium between the liquid and vapor phases in both the sample bottle and measuring cell.

Measurements commenced at $270 \mathrm{~K}$. After temperature and pressure equilibrium were obtained, the conditions were recorded. The temperature was then increased by an increment of $10 \mathrm{~K}$. As the temperature was increased, the liquid expanded, eventually filling the cell with compressed liquid. As a result, it was necessary to periodically release a small amount of liquid from the bottom of the cell to maintain a vapor space; this was done by opening a pneumatically actuated valve. Repeat measurements were conducted at a minimum of two temperatures. These repeats established the repeatability of the measurements and also helped to determine whether the loss of small amounts of the liquid phase affected the sample composition to the extent that duplicate measurements at a given temperature yielded different bubble-point pressures. In almost all instances this was not the case.

The principal sources of uncertainty in these measurements arose from the measurement of temperature, pressure, and sample composition as well as repeatability of the measurements. The SPRT was calibrated with fixed-point cells, and the standard $(k=1)$ uncertainty in temperature, including calibration, repeatability and possible temperature gradients was estimated to be $20 \mathrm{mK}$. The pressure transducers were calibrated with a piston gage, and the zero of the transducers was regularly checked. The uncertainty in the pressure was $0.005 \%$ of the full-scale pressure (i.e., $0.035 \mathrm{kPa}$ or $0.35 \mathrm{kPa}$ for the two transducers). As described in section A.1-5, the uncertainty in the composition due to the weighings was less than 0.0001 mole fraction. Because of the two-phase nature of the liquid-phase samples and because the weighings could determine only the overall (bulk) composition of the sample, it was necessary to estimate the change in composition caused by the liquid/vapor fractionation inside the cylinder. This change was less than 0.0005 mole fraction, and its standard uncertainty was estimated to be less than 0.0001 mole fraction. Thus, the combined, expanded uncertainty in composition of the liquid-phase samples was estimated to be less than 0.00028 mole fraction. In addition to these uncertainties, the standard deviation in repeat 
measurements were added in quadrature to arrive at the overall uncertainty, which is stated in the tables of VLE data presented below.

Table A.1-1. Measured vapor-liquid equilibria (VLE) data for the system R-1234yf/134a at a molar composition of $(0.320 / 0.680)$.

Listed are temperature $T$, bubble-point pressure $P_{\text {bub }}$, expanded $(k=2)$ state point uncertainty in the bubble-point pressure $U_{c}$, and relative deviation from the mixture EOS. The standard uncertainty in temperature is $20 \mathrm{mK}$, and the standard uncertainty in composition is 0.00028 mole fraction. Two replicate measurements are given for most temperatures.

$\begin{array}{crrr}T / \mathrm{K} & P_{\text {bub }} / \mathrm{kPa} & U_{\mathrm{c}} / \% & \Delta_{P \text { bub }} / \% \\ & & & \\ 269.99 & 282.7 & 0.835 & 0.111 \\ 279.99 & 399.3 & 0.614 & 0.031 \\ 279.99 & 399.6 & 0.613 & 0.106 \\ 289.99 & 549.3 & 0.466 & -0.057 \\ 289.99 & 549.6 & 0.466 & -0.002 \\ 299.99 & 739.7 & 0.362 & 0.033 \\ 299.99 & 739.8 & 0.362 & 0.046 \\ 309.99 & 975.1 & 0.286 & 0.022 \\ 309.99 & 974.5 & 0.286 & -0.039 \\ 319.99 & 1262.0 & 0.234 & -0.043 \\ 319.99 & 1261.8 & 0.234 & -0.059 \\ 329.99 & 1609.3 & 0.190 & -0.024 \\ 329.99 & 1608.6 & 0.190 & -0.068 \\ 339.99 & 2024.3 & 0.157 & -0.010 \\ 339.99 & 2023.2 & 0.157 & -0.064 \\ 349.99 & 2516.9 & 0.132 & 0.011 \\ 349.99 & 2515.1 & 0.132 & -0.061 \\ 359.99 & 3098.6 & 0.112 & -0.007 \\ 359.99 & 3095.7 & 0.112 & -0.101\end{array}$


Table A.1-2. Measured vapor-liquid equilibria (VLE) data for the system R-1234yf/134a at a molar composition of $(0.647 / 0.353)$.

Listed are temperature $T$, bubble-point pressure $P_{\text {bub }}$, expanded $(k=2)$ state point uncertainty in the bubble-point pressure $U_{c}$, and relative deviation from the mixture EOS. The standard uncertainty in temperature is $20 \mathrm{mK}$, and the standard uncertainty in composition is 0.00028 mole fraction. Two replicate measurements are given for most temperatures. Two replicate measurements are given for most temperatures.

$\begin{array}{cccc}T / \mathrm{K} & P_{\text {bub }} / \mathrm{kPa} & U_{\mathrm{c}} / \% & \Delta_{P \text { bub }} / \% \\ & & & \\ 269.99 & 289.9 & 0.793 & 0.121 \\ 269.99 & 290.0 & 0.793 & 0.155 \\ 279.99 & 406.9 & 0.587 & 0.068 \\ 279.99 & 407.0 & 0.587 & 0.093 \\ 289.99 & 557.8 & 0.443 & 0.223 \\ 289.99 & 556.5 & 0.444 & -0.010 \\ 289.99 & 556.5 & 0.444 & -0.010 \\ 299.99 & 745.2 & 0.348 & 0.052 \\ 299.99 & 745.2 & 0.348 & 0.052 \\ 309.99 & 977.0 & 0.274 & -0.024 \\ 309.99 & 976.8 & 0.274 & -0.044 \\ 319.99 & 1259.8 & 0.225 & -0.024 \\ 319.99 & 1259.3 & 0.226 & -0.063 \\ 329.99 & 1610.4 & 0.183 & 0.630 \\ 329.99 & 1599.0 & 0.184 & -0.082 \\ 339.99 & 2005.3 & 0.153 & -0.017 \\ 339.99 & 2003.8 & 0.153 & -0.092 \\ 349.99 & 2482.2 & 0.128 & -0.126 \\ 349.99 & 2482.9 & 0.128 & -0.098 \\ 359.99 & 3047.8 & 0.109 & -0.141\end{array}$


Table A.1-3. Measured vapor-liquid equilibria (VLE) data for the system $\mathrm{R}-134 \mathrm{a} / 1234 \mathrm{ze}(\mathrm{E})$ at a molar composition of $(0.334 / 0.666)$.

Listed are temperature $T$, bubble-point pressure $P_{\text {bub }}$, expanded $(k=2)$ state point uncertainty in the bubble-point pressure $U_{c}$, and relative deviation from the mixture EOS. The standard uncertainty in temperature is $20 \mathrm{mK}$, and the standard uncertainty in composition is 0.00028 mole fraction.

$\begin{array}{rrrr}T / \mathrm{K} & P_{\text {bub }} / \mathrm{kPa} & U_{\mathrm{d}} \% & \Delta_{P \text { bub }} / \% \\ & & & \\ 269.99 & 222.3 & 1.170 & -0.173 \\ 279.99 & 318.1 & 0.846 & -0.133 \\ 289.99 & 442.8 & 0.628 & -0.094 \\ 299.99 & 601.4 & 0.477 & -0.074 \\ 299.99 & 601.1 & 0.477 & -0.114 \\ 309.99 & 799.6 & 0.383 & -0.027 \\ 319.99 & 1043.7 & 0.308 & 0.051 \\ 319.99 & 1043.0 & 0.308 & -0.018 \\ 329.99 & 1339.6 & 0.249 & 0.116 \\ 339.99 & 1694.3 & 0.204 & 0.179 \\ 349.99 & 2115.3 & 0.170 & 0.218 \\ 359.99 & 2614.9 & 0.145 & 0.363 \\ 359.99 & 2609.0 & 0.145 & 0.137\end{array}$

Table A.1-4. Measured vapor-liquid equilibria (VLE) data for the system R-134a/1234ze(E) at a molar composition of $(0.663 / 0.337)$.

Listed are temperature $T$, bubble-point pressure $P_{\text {bub }}$, expanded $(k=2)$ state point uncertainty in the bubble-point pressure $U_{c}$, and relative deviation from the mixture EOS. The standard uncertainty in temperature is $20 \mathrm{mK}$, and the standard uncertainty in composition is 0.00028 mole fraction.

$\begin{array}{rrrr}T / \mathrm{K} & P_{\text {bub }} / \mathrm{kPa} & U_{\mathrm{c}} / \% & \Delta_{P \text { bub }} / \% \\ & & & \\ 269.99 & 244.0 & 0.951 & -0.222 \\ 279.99 & 348.6 & 0.694 & -0.209 \\ 289.99 & 484.6 & 0.520 & -0.195 \\ 299.99 & 657.0 & 0.399 & -0.246 \\ 309.99 & 872.8 & 0.315 & -0.200 \\ 319.99 & 1138.3 & 0.256 & -0.122 \\ 329.99 & 1460.0 & 0.207 & -0.058 \\ 339.99 & 1846.1 & 0.170 & 0.025 \\ 349.99 & 2304.5 & 0.142 & 0.077 \\ 359.99 & 2843.2 & 0.120 & 0.022 \\ 289.99 & 484.3 & 0.520 & -0.265\end{array}$


Table A.1-5. Measured vapor-liquid equilibria (VLE) data for the system R$1234 \mathrm{yf} / 1234 \mathrm{ze}(\mathrm{E})$ at a molar composition of (0.324/0.676).

Listed are temperature $T$, bubble-point pressure $P_{\text {bub }}$, expanded $(k=2)$ state point uncertainty in the bubble-point pressure $U_{c}$, and relative deviation from the mixture EOS. The standard uncertainty in temperature is $20 \mathrm{mK}$, and the standard uncertainty in composition is 0.00028 mole fraction. Two replicate measurements are given for most temperatures. Two replicate measurements are given for most temperatures.

$\begin{array}{crrr}T / \mathrm{K} & P_{\text {bub }} / \mathrm{kPa} & U_{\mathrm{c}} / \% & \Delta_{P \text { bub }} / \% \\ 270.00 & 229.5 & 0.924 & -0.110 \\ 270.00 & 229.5 & 0.924 & -0.110 \\ 280.00 & 325.4 & 0.676 & -0.044 \\ 280.00 & 325.5 & 0.676 & -0.013 \\ 290.00 & 449.1 & 0.510 & -0.023 \\ 290.00 & 449.2 & 0.510 & -0.001 \\ 300.00 & 605.7 & 0.393 & 0.020 \\ 300.00 & 605.4 & 0.393 & -0.029 \\ 310.00 & 799.6 & 0.313 & -0.029 \\ 310.00 & 799.1 & 0.313 & -0.091 \\ 320.00 & 1038.6 & 0.255 & 0.107 \\ 320.00 & 1037.1 & 0.256 & -0.038 \\ 330.00 & 1325.4 & 0.207 & 0.065 \\ 330.00 & 1324.7 & 0.208 & 0.012 \\ 340.00 & 1669.5 & 0.171 & 0.117 \\ 340.00 & 1667.8 & 0.171 & 0.015 \\ 340.00 & 1668.5 & 0.171 & 0.057 \\ 350.00 & 2077.0 & 0.143 & 0.149 \\ 350.00 & 2076.2 & 0.143 & 0.110 \\ 360.00 & 2556.7 & 0.121 & 0.157 \\ 360.00 & 2557.1 & 0.121 & 0.173\end{array}$


Table A.1-6. Measured vapor-liquid equilibria (VLE) data for the system R$1234 \mathrm{yf} / 1234 \mathrm{ze}(\mathrm{E})$ at a molar composition of $(0.638 / 0.362)$.

Listed are temperature $T$, bubble-point pressure $P_{\text {bub }}$, expanded $(k=2)$ state point uncertainty in the bubble-point pressure $U_{c}$, and relative deviation from the mixture EOS. The standard uncertainty in temperature is $20 \mathrm{mK}$, and the standard uncertainty in composition is 0.00028 mole fraction. Two replicate measurements are given for most temperatures. Two replicate measurements are given for most temperatures.

$\begin{array}{cccr}T / \mathrm{K} & P_{\text {bub }} / \mathrm{kPa} & U_{\mathrm{c}} / \% & \Delta_{P \text { bub }} / \% \\ & & & \\ 270.00 & 258.9 & 0.769 & 0.332 \\ 270.00 & 258.0 & 0.771 & -0.017 \\ 280.00 & 363.5 & 0.567 & 0.198 \\ 280.00 & 362.9 & 0.568 & 0.033 \\ 280.00 & 362.8 & 0.568 & 0.005 \\ 290.00 & 497.8 & 0.430 & 0.144 \\ 290.00 & 497.1 & 0.430 & 0.004 \\ 290.00 & 497.0 & 0.431 & -0.016 \\ 300.00 & 666.3 & 0.333 & 0.060 \\ 300.00 & 665.7 & 0.333 & -0.030 \\ 300.00 & 665.6 & 0.334 & -0.045 \\ 310.00 & 874.6 & 0.266 & 0.008 \\ 310.00 & 873.8 & 0.267 & -0.084 \\ 310.00 & 873.7 & 0.267 & -0.095 \\ 320.00 & 1128.8 & 0.219 & 0.013 \\ 320.00 & 1127.8 & 0.219 & -0.076 \\ 320.00 & 1127.7 & 0.219 & -0.085 \\ 330.00 & 1435.4 & 0.179 & 0.065 \\ 330.00 & 1434.0 & 0.179 & -0.033 \\ 330.00 & 1434.0 & 0.179 & -0.033 \\ 340.00 & 1800.0 & 0.148 & 0.064 \\ 340.00 & 1798.8 & 0.148 & -0.003 \\ 340.00 & 1799.1 & 0.148 & 0.014 \\ 350.00 & 2229.1 & 0.125 & -0.029 \\ 350.00 & 2231.3 & 0.124 & 0.070 \\ 360.00 & 2734.0 & 0.106 & -0.117 \\ 360.00 & 2738.9 & 0.106 & 0.062\end{array}$


Table A.1-7. Measured vapor-liquid equilibria (VLE) data for the system R125/1234yf at a molar composition of $(0.349 / 0.651)$.

Listed are temperature $T$, bubble-point pressure $P_{\text {bub }}$, expanded $(k=2)$ state point uncertainty in the bubble-point pressure $U_{c}$, and relative deviation from the mixture EOS. The standard uncertainty in temperature is $20 \mathrm{mK}$, and the standard uncertainty in composition is 0.00028 mole fraction.

$\begin{array}{crrr}T / \mathrm{K} & P_{\text {bub }} / \mathrm{kPa} & U_{\mathrm{c}} / \% & \Delta_{P \text { bub }} / \% \\ & & & \\ 270.00 & 393.4 & 0.536 & -0.148 \\ 270.00 & 393.8 & 0.536 & -0.046 \\ 280.00 & 542.2 & 0.404 & -0.084 \\ 290.00 & 729.5 & 0.317 & -0.032 \\ 300.00 & 959.9 & 0.251 & -0.108 \\ 300.00 & 960.4 & 0.251 & -0.056 \\ 310.00 & 1240.8 & 0.202 & -0.122 \\ 315.00 & 1401.0 & 0.186 & -0.201 \\ 315.00 & 1401.8 & 0.186 & -0.144 \\ 320.00 & 1577.9 & 0.169 & -0.154 \\ 325.00 & 1770.1 & 0.154 & -0.150 \\ 330.00 & 1979.7 & 0.140 & -0.116 \\ 330.00 & 1980.8 & 0.140 & -0.060 \\ 335.00 & 2206.6 & 0.129 & -0.112\end{array}$

Table A.1-8. Measured vapor-liquid equilibria (VLE) data for the system R-125/1234yf at a molar composition of $(0.664 / 0.336)$.

Listed are temperature $T$, bubble-point pressure $P_{\text {bub }}$, expanded $(k=2)$ state point uncertainty in the bubble-point pressure $U_{c}$, and relative deviation from the mixture EOS.

$\begin{array}{crrr}T / \mathrm{K} & P_{\text {bub }} / \mathrm{kPa} & U_{\mathrm{c}} / \% & \Delta_{P \text { bub }} / \% \\ & & & \\ 270.00 & 494.0 & 0.455 & -0.234 \\ 270.00 & 494.6 & 0.455 & -0.113 \\ 280.00 & 677.4 & 0.344 & -0.081 \\ 290.00 & 905.9 & 0.270 & -0.099 \\ 290.00 & 907.0 & 0.270 & 0.022 \\ 300.00 & 1186.1 & 0.215 & -0.195 \\ 310.00 & 1525.9 & 0.174 & -0.265 \\ 310.00 & 1527.6 & 0.174 & -0.154 \\ 315.00 & 1721.4 & 0.161 & -0.241 \\ 320.00 & 1935.0 & 0.146 & -0.211 \\ 325.00 & 2167.5 & 0.133 & -0.192 \\ 330.00 & 2421.0 & 0.122 & -0.142 \\ 330.00 & 2423.6 & 0.122 & -0.035 \\ 335.00 & 2695.5 & 0.112 & -0.121\end{array}$




\section{A.1.2. Density $(P, \rho, T, x)$ Measurements}

Instrument description. The present measurements utilized a two-sinker densimeter with a magnetic suspension coupling. This type of instrument applies the Archimedes (buoyancy) principle to provide an absolute determination of the density. This general type of instrument is described by Wagner and Kleinrahm, ${ }^{4}$ and our instrument is described in detail by McLinden and Lösch-Will. ${ }^{5}$ Briefly, two sinkers of nearly the same mass $(\sim 60 \mathrm{~g})$ and same surface area $\left(\sim 41.5 \mathrm{~cm}^{2}\right)$, but very different volumes, were each weighed with a highprecision balance while they were immersed in the sample of unknown density. The basic form of the working equation for this type of instrument gives the fluid density $\rho$ as:

$$
=\frac{\left(\begin{array}{lll}
m_{1} & m_{2}
\end{array}\right)\left(\begin{array}{ll}
W_{1} & W_{2}
\end{array}\right)}{\left(\begin{array}{ll}
V_{1} & V_{2}
\end{array}\right)},
$$

where $m$ and $V$ are the mass and volume of the sinkers, $W$ are the balance readings, and the subscripts refer to the two sinkers. One sinker was made of tantalum $(m=60.094633 \mathrm{~g}$, $\left.V=3.60872 \mathrm{~cm}^{3}\right)$ and the other of titanium $\left(m=60.075386 \mathrm{~g}, V=13.315284 \mathrm{~cm}^{3}\right)$. A magnetic suspension coupling transmitted the gravity and buoyancy forces on the sinkers to the balance, thus isolating the fluid sample from the balance. With the two-sinker method, systematic errors in the weighing and from other sources approximately cancel. Figure A.1-2 shows the entire instrument and Figure A.1-3 shows the two sinkers inside the cell.

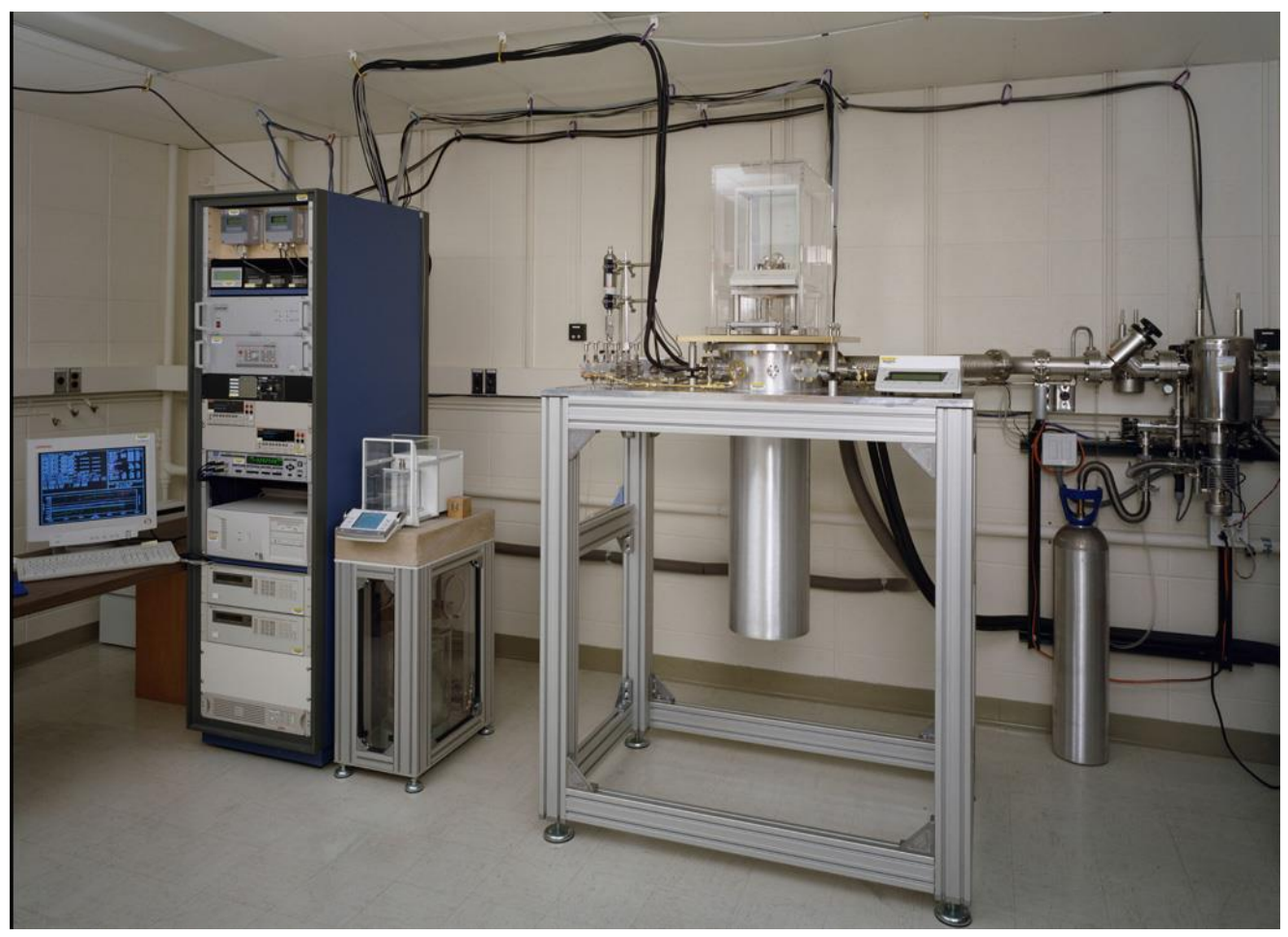

Figure A.1-2. Two-sinker densimeter.

The main part of the instrument is to the right of center with the vacuum thermostat below and balance above; the instruments are on the left side; a vacuum system for both evacuating 
the measuring cell and maintaining the vacuum thermostat is on the right. A separate system for calibrating sinker volumes sits to the right of the instrument rack.

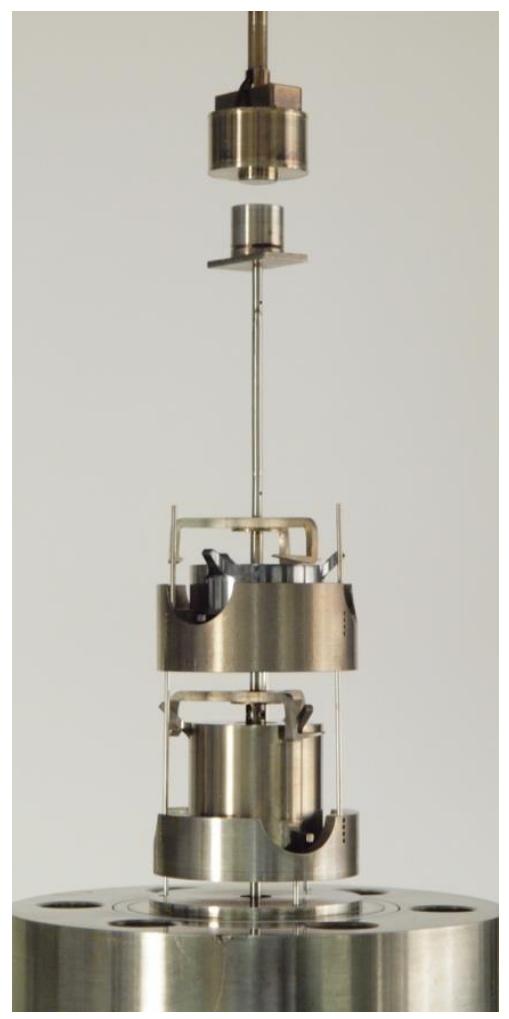

Figure A.1-3. Sinkers in the two-sinker densimeter (shown removed from the measuring cell).

The titanium sinker is on the bottom, with the tantalum sinker above. The magnetic suspension coupling is at the top of the image; this comprises the electromagnet, which hangs from the balance; and the permanent magnet, which picks up the sinkers via "lifting forks;" the top of the measuring cell passes between the electromagnet and permanent magnet. In this photo, the titanium sinker is suspended off of its rest and is being weighed.

In addition to the sinkers, two calibration masses (designated $m_{\text {cal }}$ and $m_{\text {tare }}$ ) were also weighed by placing them directly on the balance pan. This provided a calibration of the balance and also the information needed to correct for magnetic effects as described by McLinden et al. ${ }^{76}$ The four weighings (two sinkers and two calibration masses) yield a set of four equations that were solved to yield a balance calibration factor $\alpha$ and a parameter $\phi$ characterizing the efficiency of the magnetic suspension coupling. With these additional terms, the fluid density is:

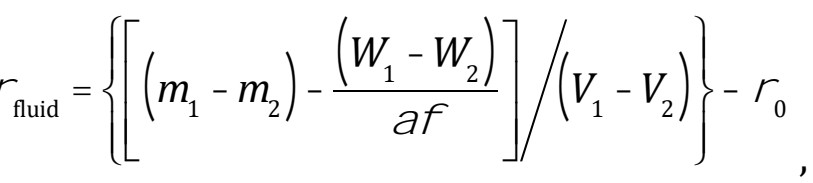


where $\rho_{0}$ is the indicated density when the sinkers are weighed in vacuum. In other words, $\rho_{0}$ is an "apparatus zero." The density given by Eq. (A.1-3) compensates for the magnetic effects of both the apparatus and the fluid being measured. The difference of the value of $\phi$ from 1 indicates the magnitude of the force transmission error. ${ }^{7}$

The densimeter was thermostated by means of a multi-layer, vacuum-insulated thermostat. A copper shield with heaters at the top and side surrounded the measuring cell. An additional isothermal shield with heaters at the top and sides and a fluid cooling channel at the top surrounded the "inner shield"; it was maintained at a temperature approximately $1 \mathrm{~K}$ below the measuring-cell temperature. A chiller that circulated ethanol was used at temperatures below room temperature.

The temperature was measured with a $25 \Omega$ standard platinum resistance thermometer (SPRT) and an AC resistance bridge referenced to a thermostated standard resistor. The temperature inside the measuring cell was constant within $5 \mathrm{mK}$. Pressures were measured with one of three vibrating-quartz-crystal type pressure transducers having full-scale pressure ranges of $2.8 \mathrm{MPa}, 13.8 \mathrm{MPa}$, or $69 \mathrm{MPa}$. The transducers and pressure manifold were thermostated at $T=313.15 \mathrm{~K}$ to minimize the effects of variations in laboratory temperature.

Density measurement sequence-liquid and supercritical states. A combination of measurements along isochores and along isotherms was carried out. The evacuated measuring cell was cooled and then the gas-phase sample from the sample bottle was condensed into the measuring cell; higher pressures were obtained by closing the valve to the sample bottle and then increasing the cell temperature in steps along a pseudoisochore. Once a new setpoint temperature and pressure was reached an additional equilibration time of 3060 minutes was allowed; four replicate density determinations were then carried out. When the maximum desired pressure along a pseudoisochore was reached, a portion of the sample was vented into a waste bottle to decrease the pressure; measurements were made in this manner along an isotherm to a minimum pressure of approximately $1 \mathrm{MPa}$ or slightly above the bubble-point pressure, whichever was higher. Measurements then resumed at increasing temperatures along the next, lower-density pseudoisochore. This procedure did not require any pump and thus avoided any chance for sample contamination that a pump or compressor might introduce; it also minimized the number of manual sample-handling steps.

Between each of the measured blend compositions and also before and after all of the testing, the densimeter cell was evacuated for a minimum of $36 \mathrm{~h}$. This was done to clear the previously measured sample and to check the zero of the pressure transducers and the $\rho_{0}$ of the apparatus (Eq. A.1-3). The $\rho_{0}$ varied by less than $0.0011 \mathrm{~kg} \cdot \mathrm{m}^{-3}$.

Vapor-phase $(P, \rho, T, x)$ and dew point. The density in the vapor phase was measured along isotherms at temperatures from $253.15 \mathrm{~K}$ to $293.15 \mathrm{~K}$. Each isotherm started at a low pressure (40 to $100 \mathrm{kPa}$ ); the pressure was increased in steps by cycling two pneumatic valves piped in series to introduce additional gaseous sample. The dew point was determined by continuing the vapor-phase isotherms into the two-phase region. As the pressure reached the dew point the value of the coupling parameter $\phi$ in Eq. (A.1-3) increased dramatically because of adsorption and condensation onto the sinkers; the intersection of lines fitted to the single-phase and two-phase data yielded the dew point. This effect and its exploitation for the 
measurement of dew points is discussed by McLinden and Richter, ${ }^{77}$ and the dew points measured here will be analyzed in the second phase of the project. With this technique the filling/pressure line was completely vapor filled up to the dew-point pressure, minimizing uncertainties in the hydrostatic head correction. An additional advantage is that a dew-point measurement is much less sensitive to the presence of a non-condensable impurity (such as air) compared to a bubble-point measurement. Each isotherm started with a fresh sample.

$(P, \rho, T, x)$ measurement uncertainties. The measurement uncertainty of the experimental density data measured with the two-sinker densimeter has been evaluated in previous works. ${ }^{5}$ 7879 Only a brief description of the main uncertainty sources is given here. The main sources of the uncertainty in density, in order of significance, arose from the sinker volumes $\left(V_{1}, V_{2}\right)$, the weighings of the sinkers and calibration masses $\left(W_{1}, W_{2}, W_{\text {cal }}, W_{\text {tare }}\right)$ and their masses $\left(m_{1}\right.$, $\left.m_{2}, m_{\text {cal }}, m_{\text {tare }}\right)$, and the apparatus zero $\rho_{0}$. The variance in the replicate balance readings was also included. The standard uncertainty in the density measurement is given by

$$
u() / \mathrm{kg} \cdot \mathrm{m}^{-3}=\left[\{28\}^{2}+\{0.20(\mathrm{~T} / \mathrm{K} \quad 293)\}^{2}+\{0.63 p / \mathrm{MPa}\}^{2}\right]^{1 / 2} \cdot 10^{6} / \mathrm{kg} \cdot \mathrm{m}^{-3}+0.0010
$$

where the term in brackets is from the uncertainty in the sinker volumes, and the final, constant term includes all other uncertainties.

The SPRT used to measure the temperature of the mixture was calibrated in our laboratory on ITS-90 from $83 \mathrm{~K}$ to $505 \mathrm{~K}$ by use of fixed-point cells. The standard uncertainty of the temperature, including the uncertainty in the fixed-point cells, drift in the SPRT and in the standard resistor, and any temperature gradients, is $3 \mathrm{mK}$. The pressure transducers were calibrated with one of two gas-operated piston gages. We estimated the standard uncertainty in pressure to be $\left(20 \times 10^{-6} \cdot p+0.03 \mathrm{kPa}\right)$ for the vapor-phase measurements and $\left(26 \times 10^{-6} \cdot p+\right.$ $1.0 \mathrm{kPa}$ ) for the liquid-phase measurements. To the above uncertainty estimates we added the standard deviations actually observed in the multiple temperature, pressure, and balance readings made over the 12 minutes necessary to complete a single density determination.

For mixtures a considerable fraction of the overall density uncertainty is due to uncertainty in the composition. This arises from the gravimetric preparation of the gas mixture used to charge the densimeter, but a larger contribution arose from possible adsorption of sample onto the inner walls of the sample cylinder, filling lines, measuring cell, etc. In other words, the composition in the measuring cell was not necessarily a simple ratio of the component masses loaded into the sample cylinder.

For purposes of comparing $(P, \rho, T, x)$ measurements to a model, it is customary to assume that the temperature, pressure, and composition are known exactly, and to lump all uncertainties into a single value for the density, a so-called state-point uncertainty. This value is tabulated in the data tables for each measured point. 
Table A.1-9. Measured $(P, \rho, T, x)$ data for the system R-1234yf/134a at a molar composition of $(0.33634 / 0.66366)$.

Listed are temperature $T$, pressure $P$, density $\rho$, standard uncertainty in pressure $u(P)$, relative combined, expanded $(k=2)$ state-point uncertainty in the density $U_{c}$, and relative deviation from the mixture EOS $\Delta \rho$. The standard uncertainty in temperature is $3 \mathrm{mK}$. Data are presented in the sequence measured. Average values for the replicate measurements at each $(T, P)$ state point are given.

\begin{tabular}{|c|c|}
\hline$T / \mathrm{K}$ & $P / \mathrm{MPa}$ \\
\hline
\end{tabular}

Vapor-phase

$\begin{array}{lrrrrr}293.154 & 0.0645 & 2.847 & 0.030 & 0.139 & -0.001 \\ 293.156 & 0.1202 & 5.369 & 0.030 & 0.078 & -0.026 \\ 293.156 & 0.1813 & 8.215 & 0.030 & 0.054 & -0.032 \\ 293.156 & 0.2435 & 11.203 & 0.030 & 0.037 & -0.038 \\ 293.156 & 0.3038 & 14.196 & 0.030 & 0.031 & -0.047 \\ 293.154 & 0.3601 & 17.079 & 0.030 & 0.027 & -0.058 \\ 293.157 & 0.4207 & 20.292 & 0.030 & 0.024 & -0.067 \\ 293.157 & 0.4801 & 23.564 & 0.030 & 0.021 & -0.081 \\ 293.157 & 0.5407 & 27.045 & 0.030 & 0.020 & -0.100 \\ 293.154 & 0.5781 & 29.102 & 0.530 & 0.172 & -0.697 \\ 283.158 & 0.0616 & 2.819 & 0.030 & 0.154 & -0.015 \\ 283.158 & 0.1024 & 4.733 & 0.030 & 0.086 & -0.024 \\ 283.156 & 0.1513 & 7.084 & 0.030 & 0.057 & -0.030 \\ 283.155 & 0.1844 & 8.710 & 0.030 & 0.047 & -0.040 \\ 283.156 & 0.2242 & 10.712 & 0.030 & 0.039 & -0.043 \\ 283.159 & 0.2682 & 12.975 & 0.030 & 0.036 & -0.051 \\ 283.159 & 0.3068 & 15.018 & 0.030 & 0.031 & -0.059 \\ 283.158 & 0.3406 & 16.850 & 0.030 & 0.027 & -0.070 \\ 283.157 & 0.3810 & 19.100 & 0.030 & 0.025 & -0.081 \\ 283.158 & 0.4105 & 20.780 & 0.030 & 0.024 & -0.092 \\ 273.156 & 0.0591 & 2.806 & 0.030 & 0.145 & -0.026 \\ 273.157 & 0.0854 & 4.087 & 0.030 & 0.099 & -0.041 \\ 273.158 & 0.1103 & 5.314 & 0.030 & 0.079 & -0.034 \\ 273.157 & 0.1557 & 7.609 & 0.030 & 0.055 & -0.045 \\ 273.156 & 0.1557 & 7.609 & 0.030 & 0.055 & -0.040 \\ 273.157 & 0.1864 & 9.198 & 0.030 & 0.052 & -0.047 \\ 273.158 & 0.2055 & 10.205 & 0.030 & 0.042 & -0.051 \\ 273.158 & 0.2323 & 11.640 & 0.030 & 0.038 & -0.058 \\ 273.156 & 0.2569 & 12.982 & 0.030 & 0.034 & -0.067\end{array}$


Table A.1-9. (continued)

$\begin{array}{crrrrr}T / \mathrm{K} & P / \mathrm{MPa} & \rho / \mathrm{kg} \cdot \mathrm{m}^{-3} & u(P) / \mathrm{kPa} & U_{\mathrm{c}} / \% & \Delta \rho / \% \\ 273.155 & 0.2866 & 14.639 & 0.030 & 0.031 & -0.078 \\ 273.157 & 0.3006 & 15.435 & 0.030 & 0.030 & -0.084 \\ 273.156 & 0.3038 & 15.614 & 0.030 & 0.029 & -0.089 \\ 273.154 & 0.3099 & 15.790 & 0.570 & 0.364 & -1.181 \\ 263.157 & 0.0460 & 2.260 & 0.030 & 0.197 & -0.047 \\ 263.158 & 0.0604 & 2.986 & 0.030 & 0.136 & -0.041 \\ 263.157 & 0.0803 & 3.993 & 0.030 & 0.103 & -0.039 \\ 263.160 & 0.1010 & 5.060 & 0.030 & 0.083 & -0.041 \\ 263.157 & 0.1207 & 6.089 & 0.030 & 0.072 & -0.046 \\ 263.159 & 0.1410 & 7.166 & 0.030 & 0.061 & -0.048 \\ 263.159 & 0.1603 & 8.206 & 0.030 & 0.057 & -0.055 \\ 263.157 & 0.1800 & 9.285 & 0.030 & 0.047 & -0.061 \\ 263.155 & 0.2014 & 10.472 & 0.030 & 0.042 & -0.074 \\ 263.155 & 0.2082 & 10.855 & 0.030 & 0.040 & -0.075 \\ 263.154 & 0.2231 & 11.427 & 0.590 & 0.577 & -99.106 \\ 253.155 & 0.0439 & 2.247 & 0.030 & 0.188 & -0.056 \\ 253.157 & 0.0615 & 3.169 & 0.030 & 0.140 & -0.053 \\ 253.156 & 0.0764 & 3.964 & 0.030 & 0.104 & -0.055 \\ 253.155 & 0.0908 & 4.738 & 0.030 & 0.089 & -0.055 \\ 253.157 & 0.1064 & 5.585 & 0.030 & 0.083 & -0.055 \\ 253.157 & 0.1214 & 6.410 & 0.030 & 0.105 & -0.082 \\ 253.158 & 0.1310 & 6.949 & 0.030 & 0.065 & -0.070\end{array}$

Compressed-liquid and supercritical states

$\begin{array}{rrrrrr}230.018 & 1.0576 & 1377.826 & 1.150 & 0.015 & -0.127 \\ 240.013 & 4.0960 & 1357.442 & 1.880 & 0.020 & -0.123 \\ 240.015 & 2.5156 & 1353.415 & 1.770 & 0.019 & -0.121 \\ 240.017 & 1.4521 & 1350.630 & 2.110 & 0.021 & -0.121 \\ 249.999 & 10.5097 & 1346.407 & 1.720 & 0.018 & -0.122 \\ 250.005 & 8.7756 & 1341.986 & 1.630 & 0.018 & -0.121 \\ 250.005 & 7.1237 & 1337.663 & 1.670 & 0.018 & -0.120 \\ 250.007 & 5.5507 & 1333.409 & 1.340 & 0.016 & -0.119 \\ 250.008 & 4.0542 & 1329.242 & 1.490 & 0.017 & -0.118 \\ 250.007 & 1.5142 & 1321.869 & 1.600 & 0.018 & -0.116 \\ 260.006 & 9.9755 & 1318.072 & 1.880 & 0.019 & -0.116 \\ 260.007 & 9.9631 & 1318.033 & 1.310 & 0.015 & -0.116 \\ 260.009 & 8.4079 & 1313.584 & 1.720 & 0.018 & -0.115 \\ 260.006 & 5.5221 & 1304.933 & 1.650 & 0.018 & -0.114 \\ 260.008 & 4.1796 & 1300.704 & 1.540 & 0.017 & -0.113\end{array}$


Table A.1-9. (continued)

$\begin{array}{rrrrrr}T / \mathrm{K} & P / \mathrm{MPa} & \rho / \mathrm{kg} \cdot \mathrm{m}^{-3} & u(P) / \mathrm{kPa} & U_{\mathrm{c}} / \% & \Delta \rho / \% \\ 260.007 & 1.6895 & 1292.499 & 1.400 & 0.016 & -0.111 \\ 269.996 & 9.5068 & 1288.959 & 1.360 & 0.015 & -0.113 \\ 269.999 & 8.1305 & 1284.487 & 1.300 & 0.015 & -0.113 \\ 269.998 & 5.5605 & 1275.750 & 1.470 & 0.016 & -0.112 \\ 270.000 & 4.3640 & 1271.459 & 1.240 & 0.015 & -0.111 \\ 269.999 & 2.1260 & 1263.066 & 1.520 & 0.016 & -0.109 \\ 280.001 & 9.3445 & 1259.774 & 1.450 & 0.016 & -0.110 \\ 280.002 & 8.1204 & 1255.273 & 1.530 & 0.016 & -0.109 \\ 280.004 & 6.9504 & 1250.820 & 1.560 & 0.017 & -0.109 \\ 280.002 & 4.7596 & 1242.075 & 1.530 & 0.016 & -0.108 \\ 280.002 & 3.7348 & 1237.760 & 1.380 & 0.015 & -0.107 \\ 280.002 & 1.8254 & 1229.313 & 1.400 & 0.016 & -0.105 \\ 295.002 & 11.6641 & 1224.821 & 1.700 & 0.017 & -0.104 \\ 295.004 & 9.4725 & 1215.562 & 1.440 & 0.016 & -0.104 \\ 295.002 & 7.4648 & 1206.491 & 1.390 & 0.015 & -0.102 \\ 295.005 & 5.6265 & 1197.556 & 1.450 & 0.016 & -0.102 \\ 295.003 & 3.9356 & 1188.754 & 1.270 & 0.015 & -0.100 \\ 295.006 & 2.3904 & 1180.086 & 1.230 & 0.015 & -0.098 \\ 309.994 & 10.9362 & 1175.984 & 1.560 & 0.016 & -0.096 \\ 309.995 & 10.0282 & 1171.275 & 1.390 & 0.015 & -0.096 \\ 309.994 & 8.3387 & 1162.044 & 1.430 & 0.016 & -0.095 \\ 309.994 & 6.0523 & 1148.363 & 1.380 & 0.015 & -0.092 \\ 309.994 & 4.0423 & 1134.895 & 1.320 & 0.015 & -0.089 \\ 309.994 & 3.4279 & 1130.444 & 1.180 & 0.014 & -0.088 \\ 325.000 & 10.8843 & 1126.954 & 1.300 & 0.015 & -0.086 \\ 325.003 & 8.7589 & 1112.710 & 1.350 & 0.015 & -0.083 \\ 325.000 & 6.3017 & 1093.916 & 1.220 & 0.015 & -0.077 \\ 325.000 & 4.2422 & 1075.416 & 1.350 & 0.016 & -0.071 \\ 325.002 & 2.5328 & 1057.236 & 1.170 & 0.015 & -0.067 \\ 339.995 & 8.5374 & 1054.188 & 1.260 & 0.015 & -0.063 \\ 339.995 & 6.7027 & 1035.338 & 1.250 & 0.015 & -0.054 \\ 339.996 & 5.1479 & 1016.459 & 1.150 & 0.015 & -0.045 \\ 339.998 & 4.1584 & 1002.423 & 1.100 & 0.015 & -0.039 \\ 339.995 & 2.5732 & 974.714 & 1.190 & 0.015 & -0.037 \\ 344.995 & 4.1226 & 973.807 & 1.120 & 0.015 & -0.023 \\ 349.993 & 5.6823 & 972.933 & 1.130 & 0.015 & -0.019 \\ 354.997 & 7.2507 & 972.074 & 1.120 & 0.015 & -0.021 \\ 359.997 & 8.8219 & 971.227 & 1.160 & 0.015 & -0.027 \\ 359.997 & 7.5859 & 952.038 & 1.090 & 0.015 & -0.010\end{array}$


Table A.1-9. (continued)

$\begin{array}{cccccc}T / \mathrm{K} & P / \mathrm{MPa} & \rho / \mathrm{kg} \cdot \mathrm{m}^{-3} & u(P) / \mathrm{kPa} & U_{\mathrm{c}} / \% & \Delta \rho / \% \\ 359.998 & 6.1084 & 923.683 & 1.100 & 0.015 & 0.017 \\ 360.000 & 4.8366 & 890.821 & 1.110 & 0.015 & 0.045 \\ 359.998 & 3.5802 & 839.396 & 1.050 & 0.015 & 0.087 \\ 364.997 & 4.5968 & 838.736 & 1.050 & 0.016 & 0.090 \\ 369.997 & 5.6301 & 838.098 & 1.050 & 0.016 & 0.082 \\ 375.000 & 6.6755 & 837.453 & 1.080 & 0.016 & 0.076 \\ 379.995 & 7.7284 & 836.812 & 1.060 & 0.016 & 0.060 \\ 379.996 & 6.8786 & 805.893 & 1.100 & 0.016 & 0.095 \\ 379.996 & 6.2346 & 774.666 & 1.050 & 0.016 & 0.132 \\ 379.997 & 5.1658 & 681.598 & 1.050 & 0.017 & 0.324 \\ 384.996 & 5.8202 & 681.229 & 1.040 & 0.017 & 0.259 \\ 389.995 & 6.4842 & 680.854 & 1.050 & 0.017 & 0.209 \\ 394.995 & 7.1547 & 680.450 & 1.050 & 0.017 & 0.179 \\ 400.001 & 7.8310 & 680.027 & 1.050 & 0.017 & 0.154 \\ 400.000 & 7.0690 & 616.988 & 1.060 & 0.018 & 0.207 \\ 399.998 & 5.9665 & 460.146 & 1.050 & 0.021 & 0.240 \\ 400.000 & 5.0347 & 305.067 & 1.070 & 0.028 & 0.072 \\ 400.000 & 3.8496 & 183.201 & 1.090 & 0.043 & -0.047 \\ 400.000 & 2.9176 & 121.603 & 1.100 & 0.043 & -0.080\end{array}$


Table A.1-10. Measured $(P, \rho, T, x)$ data for the system R-1234yf/134a at a molar composition of $(0.64709 / 0.35291)$.

Listed are temperature $T$, pressure $P$, density $\rho$, standard uncertainty in pressure $\mathrm{u}(P)$, relative combined, expanded $(\mathrm{k}=2)$ state-point uncertainty in the density Uc, and relative deviation from the mixture EOS. The standard uncertainty in temperature is $3 \mathrm{mK}$. Data are presented in the sequence measured. Average values for the replicate measurements at each $(T, P)$ state point are given.

$\begin{array}{llllll}T / \mathrm{K} & P / \mathrm{MPa} & \rho / \mathrm{kg} \cdot \mathrm{m}^{-3} & u(P) / \mathrm{kPa} & U_{\mathrm{c}} / \% & \Delta \rho / \%\end{array}$

Vapor-phase

$\begin{array}{lrrrrr}293.156 & 0.0660 & 3.024 & 0.030 & 0.126 & 0.159 \\ 293.155 & 0.1241 & 5.762 & 0.030 & 0.074 & 0.175 \\ 293.154 & 0.1874 & 8.833 & 0.030 & 0.047 & 0.179 \\ 293.155 & 0.2418 & 11.555 & 0.030 & 0.037 & 0.178 \\ 293.157 & 0.3059 & 14.865 & 0.030 & 0.030 & 0.175 \\ 293.155 & 0.3654 & 18.045 & 0.030 & 0.026 & 0.169 \\ 293.155 & 0.4237 & 21.280 & 0.030 & 0.023 & 0.157 \\ 293.155 & 0.4801 & 24.530 & 0.030 & 0.021 & 0.147 \\ 283.154 & 0.0625 & 2.968 & 0.030 & 0.134 & 0.175 \\ 283.155 & 0.1042 & 4.999 & 0.030 & 0.081 & 0.187 \\ 283.156 & 0.1422 & 6.894 & 0.030 & 0.060 & 0.183 \\ 283.158 & 0.1879 & 9.226 & 0.030 & 0.045 & 0.181 \\ 283.157 & 0.2286 & 11.356 & 0.030 & 0.037 & 0.175 \\ 283.157 & 0.2648 & 13.299 & 0.030 & 0.033 & 0.169 \\ 283.156 & 0.3048 & 15.499 & 0.030 & 0.029 & 0.162 \\ 283.155 & 0.3462 & 17.841 & 0.030 & 0.026 & 0.153 \\ 283.155 & 0.3815 & 19.892 & 0.030 & 0.024 & 0.143 \\ 283.155 & 0.4209 & 22.247 & 0.030 & 0.022 & 0.130 \\ 273.153 & 0.0674 & 3.327 & 0.030 & 0.121 & 0.157 \\ 273.152 & 0.0932 & 4.638 & 0.030 & 0.088 & 0.174 \\ 273.153 & 0.1291 & 6.498 & 0.030 & 0.063 & 0.169 \\ 273.152 & 0.1513 & 7.670 & 0.030 & 0.055 & 0.169 \\ 273.151 & 0.1823 & 9.331 & 0.030 & 0.046 & 0.165 \\ 273.152 & 0.2106 & 10.885 & 0.030 & 0.040 & 0.157 \\ 273.151 & 0.2449 & 12.804 & 0.030 & 0.035 & 0.149 \\ 273.151 & 0.2755 & 14.567 & 0.030 & 0.031 & 0.144 \\ 273.152 & 0.2965 & 15.794 & 0.030 & 0.029 & 0.137 \\ 263.156 & 0.0442 & 2.252 & 0.030 & 0.182 & 0.137 \\ 263.156 & 0.0696 & 3.581 & 0.030 & 0.114 & 0.155 \\ 263.156 & 0.0818 & 4.224 & 0.030 & 0.097 & 0.159 \\ 263.156 & 0.1050 & 5.469 & 0.030 & 0.076 & 0.161 \\ 263.155 & 0.1269 & 6.663 & 0.030 & 0.063 & 0.156\end{array}$


Table A.1-10. (continued)

$\begin{array}{ccrrrr}T / \mathrm{K} & P / \mathrm{MPa} & \rho / \mathrm{kg} \cdot \mathrm{m}^{-3} & u(P) / \mathrm{kPa} & U_{\mathrm{c}} / \% & \Delta \rho / \% \\ & & & & & \\ 263.155 & 0.1476 & 7.807 & 0.030 & 0.054 & 0.154 \\ 263.156 & 0.1671 & 8.903 & 0.030 & 0.049 & 0.151 \\ 263.154 & 0.1854 & 9.953 & 0.030 & 0.044 & 0.144 \\ 253.154 & 0.0413 & 2.189 & 0.030 & 0.191 & 0.131 \\ 253.153 & 0.0569 & 3.040 & 0.030 & 0.137 & 0.134 \\ 253.155 & 0.0718 & 3.859 & 0.030 & 0.108 & 0.141 \\ 253.153 & 0.0861 & 4.650 & 0.030 & 0.090 & 0.143 \\ 253.154 & 0.1014 & 5.514 & 0.030 & 0.076 & 0.138 \\ 253.154 & 0.1161 & 6.352 & 0.030 & 0.066 & 0.139 \\ 253.153 & 0.1302 & 7.164 & 0.030 & 0.060 & 0.138\end{array}$

Compressed-liquid and supercritical states

$\begin{array}{rrrrrr}230.002 & 0.9128 & 1336.222 & 2.220 & 0.023 & -0.296 \\ 239.998 & 1.0653 & 1309.005 & 1.270 & 0.016 & -0.293 \\ 239.998 & 1.0651 & 1309.002 & 1.190 & 0.016 & -0.293 \\ 249.997 & 5.6266 & 1294.295 & 1.880 & 0.020 & -0.290 \\ 249.998 & 4.1645 & 1290.030 & 1.930 & 0.020 & -0.290 \\ 249.999 & 1.6129 & 1282.245 & 1.770 & 0.019 & -0.290 \\ 259.997 & 9.4759 & 1278.562 & 1.630 & 0.018 & -0.284 \\ 259.999 & 7.9770 & 1274.037 & 1.500 & 0.017 & -0.285 \\ 259.997 & 5.2635 & 1265.460 & 1.300 & 0.016 & -0.285 \\ 260.000 & 3.9996 & 1261.256 & 1.280 & 0.015 & -0.286 \\ 259.999 & 1.6694 & 1253.139 & 1.430 & 0.017 & -0.286 \\ 269.994 & 8.9006 & 1249.632 & 1.880 & 0.019 & -0.278 \\ 269.997 & 7.5847 & 1245.109 & 1.420 & 0.016 & -0.279 \\ 269.997 & 6.3403 & 1240.695 & 1.540 & 0.017 & -0.279 \\ 269.998 & 4.0550 & 1232.170 & 1.490 & 0.017 & -0.281 \\ 269.998 & 1.9836 & 1223.903 & 1.440 & 0.016 & -0.282 \\ 280.001 & 8.6323 & 1220.574 & 1.520 & 0.017 & -0.270 \\ 280.001 & 7.4700 & 1216.029 & 1.430 & 0.016 & -0.271 \\ 280.001 & 5.3396 & 1207.255 & 1.400 & 0.016 & -0.273 \\ 280.003 & 3.4024 & 1198.696 & 1.270 & 0.015 & -0.275 \\ 280.003 & 1.6537 & 1190.428 & 1.490 & 0.016 & -0.277 \\ 295.003 & 10.5573 & 1185.253 & 1.400 & 0.016 & -0.251 \\ 295.004 & 9.5150 & 1180.602 & 1.420 & 0.016 & -0.253 \\ 295.003 & 7.5910 & 1171.564 & 1.330 & 0.015 & -0.255 \\ 295.006 & 5.8284 & 1162.653 & 1.370 & 0.015 & -0.257 \\ 295.003 & 4.2089 & 1153.864 & 1.550 & 0.017 & -0.260 \\ 295.003 & 2.0563 & 1141.048 & 1.290 & 0.015 & -0.264\end{array}$


Table A.1-10. (continued)

$\begin{array}{cccccc}T / \mathrm{K} & P / \mathrm{MPa} & \rho / \mathrm{kg} \cdot \mathrm{m}^{-3} & u(P) / \mathrm{kPa} & U_{\mathrm{c}} / \% & \Delta \rho / \% \\ 309.994 & 9.9069 & 1137.041 & 1.290 & 0.015 & -0.229 \\ 309.999 & 8.2737 & 1127.710 & 1.360 & 0.015 & -0.231 \\ 309.996 & 6.0974 & 1114.100 & 1.390 & 0.016 & -0.235 \\ 309.997 & 4.1899 & 1100.709 & 1.350 & 0.015 & -0.239 \\ 309.995 & 2.0299 & 1083.314 & 1.550 & 0.017 & -0.248 \\ 325.002 & 8.6630 & 1079.822 & 1.250 & 0.015 & -0.204 \\ 325.003 & 8.0388 & 1075.113 & 1.260 & 0.015 & -0.204 \\ 325.001 & 5.8320 & 1056.789 & 1.300 & 0.015 & -0.209 \\ 325.002 & 3.9881 & 1038.780 & 1.290 & 0.015 & -0.216 \\ 325.002 & 2.1196 & 1016.673 & 1.290 & 0.016 & -0.237 \\ 339.997 & 7.5364 & 1013.684 & 1.220 & 0.015 & -0.173 \\ 339.997 & 5.9387 & 995.131 & 1.110 & 0.015 & -0.176 \\ 339.997 & 4.0328 & 967.738 & 1.170 & 0.015 & -0.185 \\ 339.996 & 2.6131 & 940.709 & 1.120 & 0.015 & -0.211 \\ 344.995 & 4.0305 & 939.822 & 1.080 & 0.015 & -0.169 \\ 349.994 & 5.4565 & 938.943 & 1.130 & 0.015 & -0.145 \\ 354.994 & 6.8882 & 938.074 & 1.110 & 0.015 & -0.132 \\ 359.998 & 8.3244 & 937.210 & 1.130 & 0.015 & -0.128 \\ 359.998 & 6.4863 & 904.331 & 1.140 & 0.015 & -0.110 \\ 359.998 & 5.0076 & 867.437 & 1.070 & 0.015 & -0.086 \\ 359.999 & 3.5874 & 807.728 & 1.050 & 0.016 & -0.030 \\ 364.998 & 4.5146 & 807.093 & 1.040 & 0.016 & -0.018 \\ 369.998 & 5.4569 & 806.464 & 1.050 & 0.016 & -0.016 \\ 374.998 & 6.4088 & 805.823 & 1.060 & 0.016 & -0.019 \\ 379.998 & 7.3671 & 805.162 & 1.060 & 0.016 & -0.028 \\ 379.997 & 6.5403 & 772.196 & 1.070 & 0.016 & 0.024 \\ 379.998 & 5.5548 & 711.581 & 1.050 & 0.017 & 0.162 \\ 384.998 & 5.2432 & 595.068 & 1.040 & 0.018 & 0.665 \\ 389.997 & 5.7522 & 594.705 & 1.040 & 0.018 & 0.549 \\ 394.997 & 6.2656 & 594.329 & 1.050 & 0.018 & 0.485 \\ 399.999 & 6.7830 & 593.964 & 1.050 & 0.018 & 0.438 \\ 400.002 & 5.8987 & 475.041 & 1.050 & 0.021 & 0.718 \\ 400.001 & 4.9852 & 322.447 & 1.070 & 0.027 & 0.759 \\ 400.001 & 3.9472 & 203.238 & 1.090 & 0.040 & 0.598 \\ 400.001 & 2.7048 & 115.490 & 1.100 & 0.040 & 0.344 \\ 400.003 & 1.7401 & 66.680 & 1.110 & 0.117 & 0.139\end{array}$


Table A.1-11. Measured $(P, \rho, T, x)$ data for the system R-134a/1234ze(E) at a molar composition of $(0.33250 / 0.66750)$.

Listed are temperature $T$, pressure $P$, density $\rho$, standard uncertainty in pressure $u(P)$, relative combined, expanded $(k=2)$ state-point uncertainty in the density $U_{c}$, and relative deviation from the mixture EOS $\Delta \rho$. The standard uncertainty in temperature is $3 \mathrm{mK}$. Data are presented in the sequence measured. Average values for the replicate measurements at each $(T, P)$ state point are given.

$T / \mathrm{K} \quad P / \mathrm{MPa} \quad \rho / \mathrm{kg} \cdot \mathrm{m}^{-3} \quad u(P) / \mathrm{kPa} \quad U_{\mathrm{c}} / \% \quad \Delta \rho / \%$

Vapor-phase

$\begin{array}{lrrrrr}293.156 & 0.0577 & 2.640 & 0.030 & 0.160 & -0.028 \\ 293.156 & 0.0577 & 2.640 & 0.030 & 0.136 & -0.028 \\ 293.157 & 0.1025 & 4.739 & 0.030 & 0.086 & -0.040 \\ 293.159 & 0.1415 & 6.606 & 0.030 & 0.057 & -0.048 \\ 293.159 & 0.1817 & 8.574 & 0.030 & 0.045 & -0.056 \\ 293.157 & 0.2212 & 10.545 & 0.030 & 0.038 & -0.071 \\ 293.158 & 0.2624 & 12.649 & 0.030 & 0.033 & -0.077 \\ 293.157 & 0.3013 & 14.681 & 0.030 & 0.028 & -0.090 \\ 293.157 & 0.3417 & 16.848 & 0.030 & 0.027 & -0.102 \\ 293.159 & 0.3807 & 18.993 & 0.030 & 0.024 & -0.110 \\ 283.155 & 0.0603 & 2.864 & 0.030 & 0.129 & -0.024 \\ 283.156 & 0.0947 & 4.540 & 0.030 & 0.082 & -0.038 \\ 283.157 & 0.1255 & 6.070 & 0.030 & 0.062 & -0.043 \\ 283.155 & 0.1532 & 7.466 & 0.030 & 0.051 & -0.047 \\ 283.155 & 0.1838 & 9.042 & 0.030 & 0.043 & -0.055 \\ 283.157 & 0.2107 & 10.449 & 0.030 & 0.038 & -0.062 \\ 283.158 & 0.2430 & 12.172 & 0.030 & 0.034 & -0.067 \\ 283.157 & 0.2700 & 13.645 & 0.030 & 0.031 & -0.072 \\ 283.156 & 0.3015 & 15.394 & 0.030 & 0.029 & -0.079 \\ 283.157 & 0.3218 & 16.544 & 0.030 & 0.027 & -0.085 \\ 283.157 & 0.3241 & 16.674 & 0.030 & 0.027 & -0.085 \\ 273.158 & 0.0496 & 2.438 & 0.030 & 0.154 & -0.047 \\ 273.155 & 0.0751 & 3.720 & 0.030 & 0.108 & -0.052 \\ 273.155 & 0.1059 & 5.298 & 0.030 & 0.072 & -0.051 \\ 273.154 & 0.1269 & 6.393 & 0.030 & 0.060 & -0.049 \\ 273.155 & 0.1524 & 7.744 & 0.030 & 0.056 & -0.048 \\ 273.155 & 0.1754 & 8.986 & 0.030 & 0.044 & -0.050 \\ 273.158 & 0.2011 & 10.397 & 0.030 & 0.040 & -0.052 \\ 273.160 & 0.2238 & 11.666 & 0.030 & 0.039 & -0.055 \\ 263.161 & 0.0376 & 1.917 & 0.030 & 0.235 & -0.057 \\ 263.161 & 0.0628 & 3.225 & 0.030 & 0.122 & -0.046 \\ 263.158 & 0.0860 & 4.454 & 0.030 & 0.086 & -0.038\end{array}$


Table A.1-11. (continued)

$\begin{array}{crrrrr}T / \mathrm{K} & P / \mathrm{MPa} & \rho / \mathrm{kg} \cdot \mathrm{m}^{-3} & u(P) / \mathrm{kPa} & U_{\mathrm{c}} / \% & \Delta \rho / \% \\ & & & & & \\ 263.156 & 0.1004 & 5.231 & 0.030 & 0.082 & -0.042 \\ 263.156 & 0.1206 & 6.333 & 0.030 & 0.062 & -0.042 \\ 263.158 & 0.1452 & 7.697 & 0.030 & 0.052 & -0.036 \\ 263.160 & 0.1565 & 8.337 & 0.030 & 0.054 & -0.032 \\ 253.167 & 0.0361 & 1.913 & 0.030 & 0.202 & -0.084 \\ 253.163 & 0.0455 & 2.419 & 0.030 & 0.159 & -0.070 \\ 253.163 & 0.0606 & 3.244 & 0.030 & 0.125 & -0.037 \\ 253.163 & 0.0760 & 4.094 & 0.030 & 0.102 & -0.045 \\ 253.164 & 0.0905 & 4.906 & 0.030 & 0.081 & -0.030\end{array}$

Compressed-liquid and supercritical states

$\begin{array}{rrrrrr}230.011 & 0.9398 & 1379.400 & 2.120 & 0.021 & 0.062 \\ 230.011 & 0.9410 & 1379.400 & 2.100 & 0.021 & 0.062 \\ 230.010 & 0.9419 & 1379.406 & 1.660 & 0.018 & 0.062 \\ 245.008 & 14.0799 & 1368.702 & 1.800 & 0.018 & 0.058 \\ 245.008 & 14.0811 & 1368.706 & 1.760 & 0.018 & 0.059 \\ 245.013 & 11.8531 & 1364.114 & 1.630 & 0.017 & 0.066 \\ 245.011 & 7.7630 & 1355.310 & 1.920 & 0.019 & 0.079 \\ 245.012 & 4.0499 & 1346.804 & 1.780 & 0.018 & 0.092 \\ 245.015 & 1.3039 & 1340.152 & 1.630 & 0.017 & 0.103 \\ 259.998 & 15.4284 & 1335.162 & 1.960 & 0.019 & 0.083 \\ 260.001 & 13.4515 & 1330.555 & 1.460 & 0.016 & 0.089 \\ 260.000 & 11.5563 & 1326.008 & 1.680 & 0.017 & 0.095 \\ 260.000 & 8.0156 & 1317.092 & 1.350 & 0.015 & 0.107 \\ 260.001 & 3.2734 & 1304.149 & 1.750 & 0.018 & 0.125 \\ 260.002 & 1.8344 & 1299.957 & 1.250 & 0.015 & 0.131 \\ 279.999 & 18.6289 & 1294.065 & 1.580 & 0.016 & 0.099 \\ 280.001 & 15.1498 & 1284.619 & 1.560 & 0.016 & 0.109 \\ 280.002 & 11.9530 & 1275.346 & 1.400 & 0.015 & 0.119 \\ 280.002 & 7.6361 & 1261.735 & 1.470 & 0.016 & 0.135 \\ 280.002 & 3.8418 & 1248.486 & 1.550 & 0.016 & 0.150 \\ 280.004 & 1.5807 & 1239.867 & 1.480 & 0.016 & 0.161 \\ 304.997 & 19.1999 & 1233.418 & 1.480 & 0.015 & 0.116 \\ 304.998 & 16.3852 & 1223.766 & 1.640 & 0.016 & 0.124 \\ 304.998 & 12.5771 & 1209.525 & 1.390 & 0.015 & 0.135 \\ 304.997 & 8.1740 & 1190.906 & 1.500 & 0.016 & 0.151 \\ 304.997 & 4.4491 & 1172.670 & 1.630 & 0.016 & 0.169 \\ 304.997 & 1.3206 & 1154.826 & 1.480 & 0.016 & 0.187 \\ 309.996 & 4.0730 & 1153.572 & 1.410 & 0.015 & 0.176\end{array}$


Table A.1-11. (continued)

$\begin{array}{rrrrrr}T / \mathrm{K} & P / \mathrm{MPa} & \rho / \mathrm{kg} \cdot \mathrm{m}^{-3} & u(P) / \mathrm{kPa} & U_{\mathrm{d}} / \% & \Delta \rho / \% \\ 319.988 & 9.5781 & 1151.337 & 1.380 & 0.015 & 0.156 \\ 329.988 & 15.0585 & 1149.270 & 1.260 & 0.015 & 0.141 \\ 339.994 & 20.5178 & 1147.397 & 1.500 & 0.016 & 0.130 \\ 339.994 & 15.6051 & 1122.887 & 1.480 & 0.016 & 0.146 \\ 339.994 & 12.3123 & 1103.560 & 1.400 & 0.015 & 0.161 \\ 339.994 & 8.2775 & 1074.984 & 1.430 & 0.016 & 0.188 \\ 339.997 & 4.2837 & 1037.485 & 1.120 & 0.015 & 0.235 \\ 339.994 & 2.4419 & 1014.334 & 1.200 & 0.015 & 0.268 \\ 349.993 & 6.0374 & 1012.627 & 1.140 & 0.015 & 0.240 \\ 359.995 & 9.6560 & 1011.043 & 1.100 & 0.015 & 0.206 \\ 369.995 & 13.2786 & 1009.529 & 1.130 & 0.015 & 0.179 \\ 379.994 & 16.8942 & 1008.053 & 1.160 & 0.016 & 0.159 \\ 379.994 & 13.7847 & 978.472 & 1.200 & 0.016 & 0.178 \\ 379.994 & 10.9640 & 944.282 & 1.170 & 0.016 & 0.207 \\ 379.996 & 8.1076 & 895.878 & 1.090 & 0.016 & 0.266 \\ 379.996 & 5.0537 & 795.139 & 1.090 & 0.016 & 0.455 \\ 384.994 & 5.9657 & 794.626 & 1.060 & 0.016 & 0.381 \\ 389.994 & 6.8886 & 794.099 & 1.060 & 0.016 & 0.317 \\ 394.993 & 7.8191 & 793.564 & 1.060 & 0.016 & 0.270 \\ 399.997 & 8.7563 & 793.013 & 1.070 & 0.017 & 0.237 \\ 399.998 & 7.2821 & 727.654 & 1.080 & 0.017 & 0.252 \\ 399.998 & 6.1369 & 629.406 & 1.080 & 0.018 & 0.148 \\ 399.998 & 5.0684 & 399.697 & 1.100 & 0.022 & -1.144 \\ 399.998 & 3.8715 & 206.293 & 1.120 & 0.037 & -0.785 \\ 399.998 & 2.5928 & 110.897 & 1.130 & 0.037 & -0.431\end{array}$


Table A.1-12. Measured $(P, \rho, T, x)$ data for the system R-134a/1234ze(E) at a molar composition of $(0.66356 / 0.33644)$.

Listed are temperature $T$, pressure $P$, density $\rho$, standard uncertainty in pressure $u(P)$, relative combined, expanded $(k=2)$ state-point uncertainty in the density $U_{c}$, and relative deviation from the mixture EOS $\Delta \rho$. The standard uncertainty in temperature is $3 \mathrm{mK}$. Data are presented in the sequence measured. Average values for the replicate measurements at each $(T, P)$ state point are given.

$$
T / \mathrm{K} \quad P / \mathrm{MPa} \quad \rho / \mathrm{kg} \cdot \mathrm{m}^{-3} \quad u(P) / \mathrm{kPa} \quad U_{\mathrm{c}} / \% \quad \Delta \rho / \%
$$

Vapor-phase

$\begin{array}{lrrrrr}293.154 & 0.0537 & 2.364 & 0.030 & 0.152 & -0.057 \\ 293.157 & 0.1002 & 4.458 & 0.030 & 0.083 & -0.062 \\ 293.158 & 0.1512 & 6.808 & 0.030 & 0.059 & -0.063 \\ 293.160 & 0.2037 & 9.290 & 0.030 & 0.042 & -0.076 \\ 293.159 & 0.2550 & 11.783 & 0.030 & 0.034 & -0.092 \\ 293.159 & 0.3029 & 14.175 & 0.030 & 0.030 & -0.106 \\ 293.158 & 0.3501 & 16.597 & 0.030 & 0.026 & -0.121 \\ 293.157 & 0.4017 & 19.330 & 0.030 & 0.024 & -0.139 \\ 293.159 & 0.4503 & 21.986 & 0.030 & 0.023 & -0.159 \\ 293.158 & 0.4808 & 23.697 & 0.030 & 0.021 & -0.174 \\ 283.154 & 0.0511 & 2.329 & 0.030 & 0.175 & -0.064 \\ 283.155 & 0.1060 & 4.903 & 0.030 & 0.078 & -0.065 \\ 283.157 & 0.1536 & 7.203 & 0.030 & 0.054 & -0.072 \\ 283.157 & 0.2028 & 9.644 & 0.030 & 0.041 & -0.081 \\ 283.158 & 0.2510 & 12.108 & 0.030 & 0.034 & -0.093 \\ 283.158 & 0.3013 & 14.762 & 0.030 & 0.029 & -0.109 \\ 283.158 & 0.3528 & 17.573 & 0.030 & 0.026 & -0.135 \\ 273.158 & 0.0489 & 2.313 & 0.030 & 0.168 & -0.083 \\ 273.156 & 0.0815 & 3.895 & 0.030 & 0.101 & -0.060 \\ 273.156 & 0.1208 & 5.844 & 0.030 & 0.067 & -0.062 \\ 273.155 & 0.1640 & 8.046 & 0.030 & 0.048 & -0.073 \\ 273.157 & 0.2017 & 10.021 & 0.030 & 0.048 & -0.079 \\ 273.158 & 0.2410 & 12.141 & 0.030 & 0.035 & -0.095 \\ 263.158 & 0.0468 & 2.302 & 0.030 & 0.168 & -0.073 \\ 263.157 & 0.0780 & 3.880 & 0.030 & 0.105 & -0.067 \\ 263.156 & 0.1066 & 5.359 & 0.030 & 0.073 & -0.066 \\ 263.154 & 0.1329 & 6.745 & 0.030 & 0.058 & -0.069 \\ 263.154 & 0.1571 & 8.047 & 0.030 & 0.055 & -0.073 \\ 263.154 & 0.1722 & 8.874 & 0.030 & 0.046 & -0.075 \\ 253.162 & 0.0449 & 2.301 & 0.030 & 0.205 & -0.092 \\ 253.159 & 0.0651 & 3.361 & 0.030 & 0.129 & -0.076 \\ 253.158 & 0.0933 & 4.875 & 0.030 & 0.091 & -0.068\end{array}$


Table A.1-12. (continued)

$\begin{array}{llllll}T / \mathrm{K} & P / \mathrm{MPa} & \rho / \mathrm{kg} \cdot \mathrm{m}^{-3} & u(P) / \mathrm{kPa} & U_{\mathrm{c}} / \% & \Delta \rho / \%\end{array}$

Compressed-liquid and supercritical states

$\begin{array}{rrrrrr}230.010 & 0.9663 & 1402.623 & 4.570 & 0.037 & 0.095 \\ 230.008 & 0.9667 & 1402.628 & 5.590 & 0.044 & 0.095 \\ 230.009 & 0.9687 & 1402.626 & 4.200 & 0.034 & 0.095 \\ 245.011 & 12.0546 & 1386.704 & 1.590 & 0.017 & 0.094 \\ 245.011 & 12.0502 & 1386.694 & 1.780 & 0.018 & 0.094 \\ 245.015 & 9.9508 & 1382.143 & 1.490 & 0.016 & 0.100 \\ 245.013 & 7.9659 & 1377.720 & 1.510 & 0.017 & 0.107 \\ 245.012 & 4.2868 & 1369.139 & 1.610 & 0.017 & 0.120 \\ 245.015 & 1.4248 & 1362.070 & 1.840 & 0.018 & 0.132 \\ 260.002 & 15.7691 & 1356.809 & 1.700 & 0.017 & 0.108 \\ 260.003 & 14.0267 & 1352.679 & 1.400 & 0.016 & 0.113 \\ 260.005 & 12.1367 & 1348.071 & 1.710 & 0.017 & 0.119 \\ 260.003 & 8.6023 & 1339.042 & 1.840 & 0.018 & 0.131 \\ 260.004 & 3.8613 & 1325.928 & 1.660 & 0.017 & 0.150 \\ 260.007 & 1.3603 & 1318.456 & 1.170 & 0.015 & 0.162 \\ 280.000 & 18.3310 & 1312.269 & 1.910 & 0.018 & 0.121 \\ 280.001 & 14.9348 & 1302.734 & 1.580 & 0.016 & 0.130 \\ 280.003 & 11.8171 & 1293.387 & 1.540 & 0.016 & 0.141 \\ 280.002 & 7.6042 & 1279.684 & 1.550 & 0.016 & 0.158 \\ 280.003 & 3.8966 & 1266.338 & 1.450 & 0.016 & 0.177 \\ 280.008 & 1.6837 & 1257.638 & 1.280 & 0.015 & 0.190 \\ 304.999 & 19.5685 & 1250.996 & 1.420 & 0.015 & 0.129 \\ 304.999 & 15.4975 & 1236.449 & 1.530 & 0.016 & 0.141 \\ 304.999 & 11.8883 & 1222.160 & 1.320 & 0.015 & 0.156 \\ 304.999 & 7.7059 & 1203.446 & 1.400 & 0.015 & 0.177 \\ 304.999 & 4.1635 & 1185.110 & 1.320 & 0.015 & 0.203 \\ 304.999 & 1.8789 & 1171.601 & 1.170 & 0.014 & 0.225 \\ 314.993 & 7.4883 & 1169.120 & 1.190 & 0.014 & 0.187 \\ 339.999 & 21.4741 & 1164.055 & 1.370 & 0.015 & 0.127 \\ 339.996 & 15.6947 & 1134.447 & 1.480 & 0.016 & 0.150 \\ 339.996 & 11.8006 & 1110.170 & 1.280 & 0.015 & 0.176 \\ 339.998 & 8.0476 & 1081.430 & 1.190 & 0.015 & 0.217 \\ 339.998 & 3.9440 & 1038.952 & 1.150 & 0.015 & 0.301 \\ 339.997 & 2.3054 & 1015.611 & 1.160 & 0.015 & 0.360 \\ 349.993 & 5.8381 & 1013.890 & 1.090 & 0.015 & 0.295 \\ 359.998 & 9.4056 & 1012.324 & 1.090 & 0.015 & 0.238 \\ 369.995 & 12.9837 & 1010.853 & 1.180 & 0.015 & 0.193 \\ 379.996 & 16.5642 & 1009.426 & 1.140 & 0.016 & 0.159\end{array}$


Table A.1-12. (continued)

$\begin{array}{crrrrr}T / \mathrm{K} & P / \mathrm{MPa} & \rho / \mathrm{kg} \cdot \mathrm{m}^{-3} & u(P) / \mathrm{kPa} & U_{\mathrm{c}} \% & \Delta \rho / \% \\ 379.997 & 14.1225 & 984.560 & 1.210 & 0.016 & 0.186 \\ 379.997 & 11.0710 & 945.135 & 1.110 & 0.016 & 0.238 \\ 379.998 & 7.9933 & 886.503 & 1.080 & 0.016 & 0.339 \\ 379.998 & 5.1088 & 765.284 & 1.070 & 0.016 & 0.608 \\ 384.995 & 5.9414 & 764.838 & 1.050 & 0.016 & 0.491 \\ 389.995 & 6.7865 & 764.397 & 1.060 & 0.016 & 0.408 \\ 394.995 & 7.6408 & 763.955 & 1.060 & 0.017 & 0.346 \\ 399.999 & 8.5025 & 763.500 & 1.070 & 0.017 & 0.301 \\ 399.998 & 6.0366 & 536.798 & 1.780 & 0.026 & -0.164 \\ 400.003 & 3.7066 & 175.446 & 1.120 & 0.042 & -0.615 \\ 400.001 & 1.5024 & 54.037 & 1.140 & 0.131 & -0.361\end{array}$


Table A.1-13. Measured $(P, \rho, T, x)$ data for the system R-1234yf/1234ze(E) at a molar composition of $(0.33584 / 0.66416)$.

Listed are temperature $T$, pressure $P$, density $\rho$, standard uncertainty in pressure $u(P)$, relative combined, expanded $(k=2)$ state-point uncertainty in the density $U_{c}$, and relative deviation from the mixture EOS $\Delta \rho$. The standard uncertainty in temperature is $3 \mathrm{mK}$. Data are presented in the sequence measured. Average values for the replicate measurements at each $(T, P)$ state point are given.

$$
T / \mathrm{K} \quad P / \mathrm{MPa} \quad \rho / \mathrm{kg} \cdot \mathrm{m}^{-3} \quad u(P) / \mathrm{kPa} \quad U_{\mathrm{c}} / \% \quad \Delta \rho / \%
$$

Vapor-phase

$\begin{array}{lrrrrr}293.153 & 0.1061 & 5.092 & 0.030 & 0.075 & -0.050 \\ 293.154 & 0.1509 & 7.323 & 0.030 & 0.060 & -0.051 \\ 293.158 & 0.2004 & 9.856 & 0.030 & 0.042 & -0.056 \\ 293.159 & 0.2535 & 12.651 & 0.030 & 0.035 & -0.062 \\ 293.158 & 0.3023 & 15.303 & 0.030 & 0.029 & -0.072 \\ 293.156 & 0.3517 & 18.067 & 0.030 & 0.026 & -0.076 \\ 293.156 & 0.3755 & 19.431 & 0.030 & 0.025 & -0.079 \\ 293.156 & 0.4052 & 21.172 & 0.030 & 0.023 & -0.082 \\ 293.158 & 0.4144 & 21.715 & 0.030 & 0.023 & -0.081 \\ 293.159 & 0.4193 & 22.010 & 0.030 & 0.023 & -0.079 \\ 283.150 & 0.0765 & 3.784 & 0.030 & 0.104 & -0.054 \\ 283.150 & 0.1235 & 6.190 & 0.030 & 0.064 & -0.047 \\ 283.150 & 0.1635 & 8.289 & 0.030 & 0.049 & -0.049 \\ 283.150 & 0.2026 & 10.400 & 0.030 & 0.040 & -0.048 \\ 283.150 & 0.2436 & 12.667 & 0.030 & 0.034 & -0.053 \\ 283.150 & 0.2626 & 13.743 & 0.030 & 0.032 & -0.052 \\ 283.152 & 0.2826 & 14.887 & 0.030 & 0.031 & -0.049 \\ 283.153 & 0.3025 & 16.048 & 0.030 & 0.028 & -0.050 \\ 283.153 & 0.3172 & 16.913 & 0.030 & 0.027 & -0.048 \\ 283.154 & 0.3319 & 17.791 & 0.030 & 0.026 & -0.046 \\ 273.157 & 0.0565 & 2.884 & 0.030 & 0.143 & -0.095 \\ 273.156 & 0.0813 & 4.182 & 0.030 & 0.098 & -0.070 \\ 273.156 & 0.1041 & 5.396 & 0.030 & 0.076 & -0.057 \\ 273.158 & 0.1251 & 6.533 & 0.030 & 0.068 & -0.048 \\ 273.157 & 0.1506 & 7.934 & 0.030 & 0.055 & -0.047 \\ 273.157 & 0.1789 & 9.520 & 0.030 & 0.045 & -0.040 \\ 273.158 & 0.2038 & 10.942 & 0.030 & 0.041 & -0.037 \\ 273.159 & 0.2259 & 12.228 & 0.030 & 0.036 & -0.030 \\ 263.155 & 0.0592 & 3.149 & 0.030 & 0.129 & -0.067 \\ 263.153 & 0.0824 & 4.421 & 0.030 & 0.093 & -0.056 \\ 263.154 & 0.1038 & 5.616 & 0.030 & 0.077 & -0.046 \\ 263.155 & 0.1236 & 6.739 & 0.030 & 0.063 & -0.036\end{array}$


Table A.1-13. (continued)

$\begin{array}{crrrrr}T / \mathrm{K} & P / \mathrm{MPa} & \rho / \mathrm{kg} \cdot \mathrm{m}^{-3} & u(P) / \mathrm{kPa} & U_{\mathrm{c}} / \% & \Delta \rho / \% \\ & & & & & \\ 263.156 & 0.1420 & 7.797 & 0.030 & 0.054 & -0.027 \\ 253.155 & 0.0395 & 2.173 & 0.030 & 0.191 & -0.082 \\ 253.152 & 0.0631 & 3.506 & 0.030 & 0.119 & -0.060 \\ 253.153 & 0.0849 & 4.760 & 0.030 & 0.091 & -0.031 \\ 253.153 & 0.1050 & 5.942 & 0.030 & 0.071 & -0.024\end{array}$

Compressed-liquid and supercritical states

$\begin{array}{rrrrrr}230.007 & 0.8784 & 1338.848 & 1.230 & 0.016 & 0.046 \\ 230.004 & 0.8783 & 1338.855 & 1.250 & 0.016 & 0.046 \\ 240.002 & 6.0634 & 1325.128 & 2.100 & 0.022 & 0.054 \\ 240.004 & 4.2970 & 1321.033 & 1.400 & 0.017 & 0.057 \\ 240.008 & 2.6142 & 1317.007 & 1.460 & 0.017 & 0.060 \\ 240.003 & 1.2209 & 1313.598 & 1.480 & 0.017 & 0.062 \\ 240.001 & 1.2191 & 1313.601 & 1.610 & 0.018 & 0.062 \\ 250.000 & 10.2447 & 1310.027 & 1.480 & 0.017 & 0.060 \\ 250.002 & 8.6454 & 1306.194 & 1.480 & 0.017 & 0.063 \\ 250.001 & 5.2840 & 1297.786 & 2.080 & 0.021 & 0.069 \\ 250.001 & 2.2462 & 1289.697 & 1.780 & 0.019 & 0.074 \\ 250.001 & 1.2160 & 1286.830 & 1.750 & 0.019 & 0.075 \\ 259.997 & 9.4609 & 1283.176 & 1.740 & 0.018 & 0.073 \\ 259.999 & 7.8656 & 1278.858 & 1.380 & 0.016 & 0.075 \\ 259.999 & 6.3383 & 1274.596 & 1.540 & 0.017 & 0.077 \\ 259.997 & 3.4945 & 1266.270 & 1.650 & 0.018 & 0.081 \\ 259.999 & 1.2243 & 1259.202 & 1.740 & 0.019 & 0.085 \\ 269.995 & 8.9145 & 1255.977 & 1.660 & 0.017 & 0.082 \\ 269.996 & 7.4945 & 1251.634 & 1.300 & 0.015 & 0.084 \\ 269.999 & 6.1360 & 1247.333 & 1.480 & 0.016 & 0.086 \\ 269.995 & 3.5968 & 1238.910 & 1.520 & 0.017 & 0.089 \\ 269.998 & 1.3080 & 1230.781 & 1.450 & 0.016 & 0.091 \\ 280.000 & 8.3907 & 1227.760 & 1.350 & 0.015 & 0.091 \\ 280.003 & 7.1400 & 1223.389 & 1.350 & 0.015 & 0.092 \\ 280.002 & 4.7940 & 1214.791 & 1.480 & 0.016 & 0.094 \\ 280.004 & 2.6525 & 1206.367 & 1.240 & 0.015 & 0.096 \\ 280.004 & 1.6537 & 1202.235 & 1.430 & 0.016 & 0.096 \\ 295.003 & 11.4293 & 1198.217 & 1.320 & 0.015 & 0.100 \\ 295.003 & 9.1249 & 1189.218 & 1.320 & 0.015 & 0.101 \\ 295.005 & 6.0230 & 1175.988 & 1.240 & 0.015 & 0.102 \\ 295.006 & 4.1645 & 1167.315 & 1.200 & 0.014 & 0.102 \\ 295.005 & 1.6701 & 1154.569 & 1.250 & 0.015 & 0.100\end{array}$


Table A.1-13. (continued)

$\begin{array}{crrrrr}T / \mathrm{K} & P / \mathrm{MPa} & \rho / \mathrm{kg}^{-\mathrm{m}^{-3}} & u(P) / \mathrm{kPa} & U_{\mathrm{c}} / \% & \Delta \rho / \% \\ 310.001 & 10.1305 & 1150.870 & 1.280 & 0.015 & 0.114 \\ 310.003 & 8.2970 & 1141.824 & 1.120 & 0.014 & 0.114 \\ 310.002 & 5.8188 & 1128.430 & 1.210 & 0.015 & 0.112 \\ 310.005 & 4.3376 & 1119.616 & 1.200 & 0.015 & 0.110 \\ 310.000 & 1.7391 & 1102.274 & 1.250 & 0.015 & 0.102 \\ 314.997 & 4.1433 & 1101.096 & 1.160 & 0.014 & 0.113 \\ 319.994 & 6.5521 & 1100.014 & 1.100 & 0.014 & 0.122 \\ 324.992 & 8.9552 & 1098.971 & 1.160 & 0.014 & 0.131 \\ 324.993 & 6.8863 & 1085.356 & 1.130 & 0.014 & 0.129 \\ 324.994 & 5.0663 & 1071.889 & 1.170 & 0.014 & 0.126 \\ 324.994 & 3.4730 & 1058.570 & 1.170 & 0.015 & 0.120 \\ 324.995 & 1.6643 & 1041.020 & 1.090 & 0.014 & 0.106 \\ 329.991 & 3.6505 & 1040.015 & 1.120 & 0.014 & 0.127 \\ 334.990 & 5.6441 & 1039.089 & 1.140 & 0.015 & 0.142 \\ 339.997 & 7.6437 & 1038.200 & 1.150 & 0.015 & 0.151 \\ 339.998 & 6.1682 & 1024.498 & 1.100 & 0.014 & 0.151 \\ 339.999 & 4.8763 & 1010.886 & 1.110 & 0.014 & 0.149 \\ 340.000 & 3.4110 & 992.892 & 1.100 & 0.015 & 0.143 \\ 339.999 & 1.9412 & 970.611 & 1.150 & 0.015 & 0.124 \\ 349.995 & 5.1155 & 968.963 & 1.090 & 0.015 & 0.172 \\ 359.999 & 8.3222 & 967.480 & 1.080 & 0.015 & 0.178 \\ 360.000 & 7.2697 & 953.571 & 1.110 & 0.015 & 0.184 \\ 360.001 & 6.0691 & 935.147 & 1.080 & 0.015 & 0.193 \\ 360.002 & 5.0693 & 916.771 & 1.070 & 0.015 & 0.204 \\ 360.001 & 4.0665 & 893.911 & 1.080 & 0.015 & 0.223 \\ 370.001 & 6.5744 & 892.621 & 1.060 & 0.015 & 0.204 \\ 379.996 & 9.1111 & 891.418 & 1.070 & 0.015 & 0.173 \\ 379.997 & 7.5159 & 858.682 & 1.100 & 0.016 & 0.191 \\ 380.000 & 6.0718 & 816.850 & 1.070 & 0.016 & 0.227 \\ 379.998 & 5.0507 & 770.576 & 1.070 & 0.016 & 0.192 \\ 380.000 & 4.0343 & 659.766 & 1.070 & 0.017 & 0.172 \\ 384.996 & 4.6155 & 659.407 & 1.050 & 0.017 & 0.117 \\ 389.997 & 5.2102 & 659.054 & 1.050 & 0.017 & 0.090 \\ 394.994 & 5.8136 & 658.681 & 1.050 & 0.017 & 0.203 \\ 400.001 & 6.4252 & 658.305 & 1.080 & 0.018 & 0.185 \\ 400.001 & 5.4776 & 532.489 & 1.060 & 0.019 & -0.032 \\ 400.002 & 4.1094 & 250.120 & 1.090 & 0.033 & -0.514 \\ 399.997 & 2.7673 & 127.701 & 1.100 & 0.033 & -0.284 \\ 400.005 & 1.6855 & 67.553 & 1.110 & 0.115 & -0.309 \\ 400.002 & 0.9735 & 36.215 & 1.520 & 0.405 & -0.408\end{array}$


Table A.1-14. Measured $(P, \rho, T, x)$ data for the system R-1234yf/1234ze(E) at a molar composition of $(0.66660 / 0.33340)$.

Listed are temperature $T$, pressure $P$, density $\rho$, standard uncertainty in pressure $u(P)$, relative combined, expanded $(k=2)$ state-point uncertainty in the density $U_{c}$, and relative deviation from the mixture EOS $\Delta \rho$. The standard uncertainty in temperature is $3 \mathrm{mK}$. Data are presented in the sequence measured. Average values for the replicate measurements at each $(T, P)$ state point are given.

$T / \mathrm{K} \quad P / \mathrm{MPa} \quad \rho / \mathrm{kg} \cdot \mathrm{m}^{-3} \quad u(P) / \mathrm{kPa} \quad U_{\mathrm{c}} / \% \quad \Delta \rho / \%$

Vapor-phase

$\begin{array}{lrrrrr}293.149 & 0.0630 & 2.989 & 0.030 & 0.129 & -0.033 \\ 293.149 & 0.1234 & 5.943 & 0.030 & 0.066 & -0.037 \\ 293.150 & 0.1856 & 9.084 & 0.030 & 0.051 & -0.050 \\ 293.150 & 0.2442 & 12.136 & 0.030 & 0.035 & -0.061 \\ 293.151 & 0.3014 & 15.222 & 0.030 & 0.031 & -0.078 \\ 293.153 & 0.3596 & 18.472 & 0.030 & 0.025 & -0.094 \\ 293.153 & 0.4200 & 21.977 & 0.030 & 0.025 & -0.110 \\ 281.157 & 0.0508 & 2.512 & 0.030 & 0.194 & -0.040 \\ 281.157 & 0.1027 & 5.151 & 0.030 & 0.089 & -0.044 \\ 281.157 & 0.1533 & 7.806 & 0.030 & 0.052 & -0.055 \\ 281.156 & 0.2005 & 10.359 & 0.030 & 0.040 & -0.065 \\ 281.158 & 0.2515 & 13.204 & 0.030 & 0.038 & -0.075 \\ 281.161 & 0.3025 & 16.160 & 0.030 & 0.032 & -0.086 \\ 281.160 & 0.3251 & 17.506 & 0.030 & 0.027 & -0.087 \\ 281.160 & 0.3451 & 18.717 & 0.030 & 0.025 & -0.089 \\ 273.156 & 0.0564 & 2.879 & 0.030 & 0.173 & -0.055 \\ 273.155 & 0.0900 & 4.640 & 0.030 & 0.088 & -0.053 \\ 273.156 & 0.1201 & 6.254 & 0.030 & 0.069 & -0.052 \\ 273.156 & 0.1595 & 8.420 & 0.030 & 0.050 & -0.061 \\ 273.155 & 0.1933 & 10.320 & 0.030 & 0.048 & -0.067 \\ 273.158 & 0.2131 & 11.461 & 0.030 & 0.039 & -0.068 \\ 273.153 & 0.2310 & 12.503 & 0.030 & 0.038 & -0.075 \\ 273.154 & 0.2511 & 13.692 & 0.030 & 0.033 & -0.073 \\ 263.154 & 0.0523 & 2.775 & 0.030 & 0.149 & -0.068 \\ 263.155 & 0.0770 & 4.120 & 0.030 & 0.099 & -0.054 \\ 263.155 & 0.1069 & 5.786 & 0.030 & 0.077 & -0.055 \\ 263.154 & 0.1207 & 6.567 & 0.030 & 0.063 & -0.056 \\ 263.152 & 0.1401 & 7.678 & 0.030 & 0.055 & -0.056 \\ 263.152 & 0.1637 & 9.054 & 0.030 & 0.049 & -0.064 \\ 263.151 & 0.1799 & 10.015 & 0.030 & 0.043 & -0.062 \\ 253.155 & 0.0368 & 2.019 & 0.030 & 0.212 & -0.077 \\ 253.152 & 0.0614 & 3.408 & 0.030 & 0.126 & -0.062\end{array}$


Table A.1-14. (continued)

$\begin{array}{crrrrr}T / \mathrm{K} & P / \mathrm{MPa} & \rho / \mathrm{kg} \cdot \mathrm{m}^{-3} & u(P) / \mathrm{kPa} & U_{\mathrm{c}} / \% & \Delta_{\rho} / \% \\ & & & & & \\ 253.152 & 0.0842 & 4.714 & 0.030 & 0.095 & -0.059 \\ 253.152 & 0.1051 & 5.941 & 0.030 & 0.071 & -0.058 \\ 253.154 & 0.1102 & 6.246 & 0.030 & 0.074 & -0.060 \\ 253.157 & 0.1202 & 6.842 & 0.030 & 0.063 & -0.057\end{array}$

Compressed-liquid and supercritical states

$\begin{array}{rrrrrr}230.014 & 0.9578 & 1320.352 & 1.630 & 0.019 & 0.070 \\ 230.012 & 0.9575 & 1320.359 & 1.500 & 0.018 & 0.070 \\ 230.010 & 0.9583 & 1320.366 & 1.670 & 0.019 & 0.070 \\ 240.008 & 5.5444 & 1305.661 & 1.480 & 0.017 & 0.070 \\ 240.011 & 5.5463 & 1305.663 & 1.340 & 0.016 & 0.071 \\ 240.013 & 3.9585 & 1301.686 & 2.170 & 0.023 & 0.074 \\ 240.011 & 2.4590 & 1297.830 & 1.400 & 0.017 & 0.077 \\ 240.010 & 1.2698 & 1294.682 & 4.020 & 0.038 & 0.080 \\ 250.004 & 9.6755 & 1290.781 & 1.790 & 0.019 & 0.072 \\ 250.006 & 8.0610 & 1286.603 & 1.340 & 0.016 & 0.074 \\ 250.004 & 5.0435 & 1278.444 & 1.660 & 0.018 & 0.080 \\ 250.007 & 2.3030 & 1270.555 & 1.400 & 0.017 & 0.085 \\ 250.007 & 1.2491 & 1267.385 & 2.250 & 0.023 & 0.087 \\ 260.000 & 9.0427 & 1263.765 & 1.520 & 0.017 & 0.081 \\ 260.001 & 7.6043 & 1259.563 & 1.210 & 0.015 & 0.083 \\ 260.004 & 6.2283 & 1255.416 & 1.370 & 0.016 & 0.085 \\ 260.002 & 2.4583 & 1243.330 & 1.860 & 0.019 & 0.092 \\ 260.003 & 1.3290 & 1239.466 & 1.250 & 0.015 & 0.094 \\ 269.998 & 8.5423 & 1236.120 & 1.580 & 0.017 & 0.090 \\ 269.999 & 7.2638 & 1231.889 & 1.380 & 0.016 & 0.092 \\ 270.001 & 6.0400 & 1227.696 & 1.540 & 0.017 & 0.094 \\ 269.997 & 3.7481 & 1219.475 & 1.490 & 0.017 & 0.097 \\ 270.002 & 1.6598 & 1211.443 & 1.280 & 0.015 & 0.100 \\ 279.999 & 8.3244 & 1208.371 & 1.500 & 0.016 & 0.100 \\ 280.003 & 7.1923 & 1204.103 & 1.220 & 0.015 & 0.101 \\ 280.001 & 5.0620 & 1195.688 & 1.300 & 0.015 & 0.104 \\ 280.004 & 3.1089 & 1187.411 & 1.250 & 0.015 & 0.106 \\ 280.001 & 1.3268 & 1179.339 & 1.580 & 0.017 & 0.108 \\ 295.004 & 10.4177 & 1175.186 & 1.340 & 0.015 & 0.117 \\ 295.003 & 8.3964 & 1166.449 & 1.410 & 0.016 & 0.119 \\ 295.004 & 7.4455 & 1162.109 & 1.230 & 0.015 & 0.120 \\ 295.004 & 5.6599 & 1153.532 & 1.250 & 0.015 & 0.122 \\ 295.005 & 3.2518 & 1140.885 & 1.250 & 0.015 & 0.123\end{array}$


Table A.1-14. (continued)

$\begin{array}{rrrrrr}T / \mathrm{K} & P / \mathrm{MPa} & \rho / \mathrm{kg} \cdot \mathrm{m}^{-3} & u(P) / \mathrm{kPa} & U_{\mathrm{c}} / \% & \Delta \rho / \% \\ & & & & & \\ 295.004 & 2.5156 & 1136.726 & 1.220 & 0.015 & 0.123 \\ 309.998 & 10.5000 & 1132.914 & 1.340 & 0.015 & 0.142 \\ 310.002 & 8.8111 & 1124.052 & 1.320 & 0.015 & 0.144 \\ 310.000 & 6.5191 & 1110.918 & 1.360 & 0.016 & 0.146 \\ 310.001 & 4.4981 & 1097.962 & 1.230 & 0.015 & 0.147 \\ 310.004 & 3.2886 & 1089.421 & 1.170 & 0.015 & 0.146 \\ 310.000 & 1.6602 & 1076.765 & 1.490 & 0.017 & 0.142 \\ 314.996 & 3.8770 & 1075.564 & 1.360 & 0.016 & 0.155 \\ 319.993 & 6.0927 & 1074.429 & 1.220 & 0.015 & 0.166 \\ 324.992 & 8.3118 & 1073.367 & 1.260 & 0.015 & 0.174 \\ 324.994 & 7.0981 & 1064.512 & 1.350 & 0.016 & 0.176 \\ 324.994 & 4.9574 & 1046.987 & 1.300 & 0.015 & 0.177 \\ 324.996 & 3.5777 & 1033.973 & 1.220 & 0.015 & 0.175 \\ 324.994 & 1.6530 & 1012.488 & 1.450 & 0.017 & 0.159 \\ 329.990 & 3.4644 & 1011.475 & 1.160 & 0.015 & 0.184 \\ 334.990 & 5.2816 & 1010.507 & 1.160 & 0.015 & 0.200 \\ 339.996 & 7.1043 & 1009.576 & 1.100 & 0.014 & 0.208 \\ 339.998 & 5.8662 & 996.239 & 1.100 & 0.015 & 0.212 \\ 340.000 & 5.1260 & 987.381 & 1.190 & 0.015 & 0.213 \\ 339.999 & 3.5439 & 965.403 & 1.210 & 0.015 & 0.211 \\ 339.999 & 2.0871 & 939.259 & 1.150 & 0.015 & 0.200 \\ 349.997 & 4.9567 & 937.639 & 1.130 & 0.015 & 0.247 \\ 360.000 & 7.8541 & 936.080 & 1.140 & 0.015 & 0.242 \\ 360.002 & 7.2613 & 927.025 & 1.130 & 0.015 & 0.249 \\ 360.001 & 5.9880 & 904.517 & 1.170 & 0.016 & 0.269 \\ 360.001 & 4.9740 & 882.133 & 1.090 & 0.015 & 0.295 \\ 360.001 & 4.0423 & 855.376 & 1.060 & 0.015 & 0.338 \\ 364.999 & 5.1397 & 854.591 & 1.050 & 0.015 & 0.315 \\ 369.997 & 6.2494 & 853.933 & 1.080 & 0.016 & 0.289 \\ 374.999 & 7.3667 & 853.266 & 1.060 & 0.016 & 0.259 \\ 379.996 & 8.4858 & 852.569 & 1.130 & 0.016 & 0.231 \\ 379.998 & 7.5529 & 829.783 & 1.090 & 0.016 & 0.254 \\ 380.001 & 7.0689 & 815.792 & 1.050 & 0.016 & 0.270 \\ 380.000 & 6.0517 & 778.564 & 1.060 & 0.016 & 0.326 \\ 379.999 & 5.0385 & 718.365 & 1.050 & 0.017 & 0.431 \\ 384.997 & 4.7731 & 602.491 & 1.050 & 0.018 & 0.548 \\ 389.997 & 5.2796 & 602.119 & 1.050 & 0.018 & 0.451 \\ 394.994 & 5.7916 & 601.753 & 1.050 & 0.018 & 0.400 \\ 399.998 & 6.3086 & 601.368 & 1.050 & 0.018 & 0.370\end{array}$


Table A.1-14. (continued)

$\begin{array}{crrrrr}T / \mathrm{K} & P / \mathrm{MPa} & \rho / \mathrm{kg} \cdot \mathrm{m}^{-3} & u(P) / \mathrm{kPa} & U_{\mathrm{c}} / \% & \Delta \rho / \% \\ & & & & & \\ 400.002 & 5.5254 & 486.233 & 1.060 & 0.020 & 0.153 \\ 400.003 & 5.0718 & 394.718 & 1.070 & 0.023 & -0.169 \\ 400.002 & 4.3633 & 273.239 & 1.080 & 0.031 & -0.186 \\ 400.004 & 3.8560 & 213.394 & 1.090 & 0.038 & -0.099 \\ 400.002 & 3.1672 & 153.911 & 1.100 & 0.039 & -0.092 \\ 400.005 & 2.7375 & 124.515 & 1.100 & 0.039 & -0.138 \\ 400.004 & 2.2406 & 95.385 & 1.110 & 0.039 & -0.193 \\ 400.005 & 1.6796 & 67.018 & 1.110 & 0.115 & -0.230\end{array}$

\section{A.1.3. Liquid-Phase Speed of Sound}

Speed of sound instrument description. The speed of sound was measured over wide ranges of temperature and pressure in a dual-path, pulse-echo-type instrument. In this technique, a piezoelectric transducer is located within a sample volume of the test fluid. It is excited with a sinusoidal burst, near the crystal resonance frequency, thus emitting ultrasonic pulses from each face of the crystal, which travel through the fluid sample, reflect off planar surfaces at each end of the sample volume, and return to the transducer, which also serves as the detector. The difference in the arrival times of the echo signals give the speed of sound by

$$
w=\frac{2\left(L_{2}-L_{1}\right)}{\Delta t}
$$

where $w$ is the speed of sound, $L_{1}$ and $L_{2}$ are the path lengths, and $\Delta t$ is the time difference. The differential nature of this technique cancels end effects and improves the accuracy.

A quartz crystal with a diameter of $24 \mathrm{~mm}$, thickness of $0.36 \mathrm{~mm}$, and resonant frequency of $8.000 \mathrm{MHz}$ served as the ultrasonic transducer. The quartz crystal is "X-cut," which means that its thickness expands and contracts when a voltage is applied to electrodes on opposite faces of the crystal. It was excited with a 10-cycle sinusoidal burst from an arbitrary function generator. The fluid path lengths on the opposite faces of the crystal were $30 \mathrm{~mm}$ and $12 \mathrm{~mm}$ (ratio of 2.5:1); these separations of the crystal and the reflectors were provided by tubular spacers fabricated of a machinable ceramic. A high-speed switch connected the crystal to the function generator during the input sinusoidal burst and then, after a delay of $6 \mu \mathrm{s}$, switched the crystal to the input of a three-stage amplifier ( $5 \times$ per stage for a total of $125 \times$ ), which then fed into a digital storage oscilloscope. The echo signals were recorded for off-line analysis. 


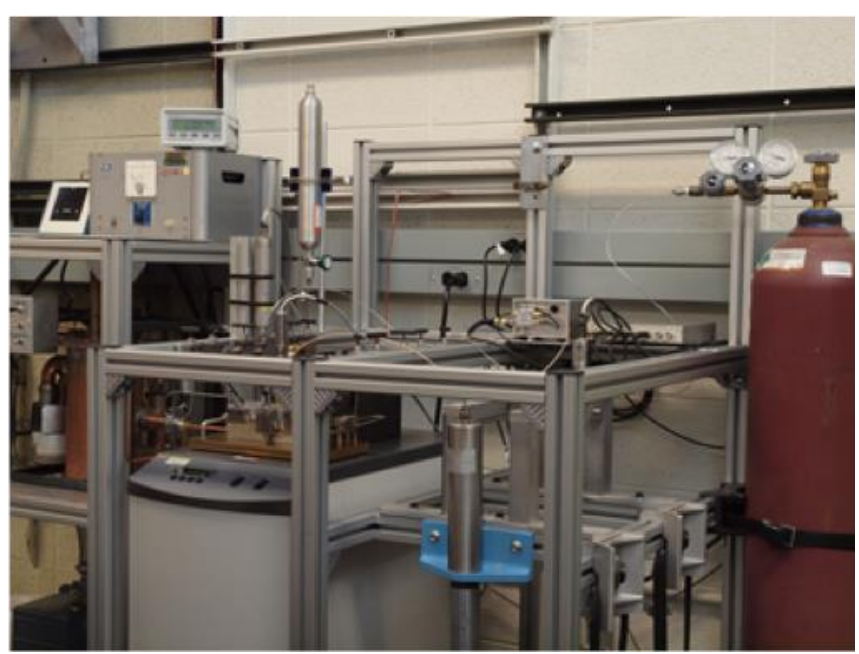

Figure A.1-4. Dual-path, pulse-echo, speed of sound instrument.

The figure shows the thermostat (left of center, which contains the measuring cell) and the fluid manifold and pressure transducer, and a vacuum system for evacuating the measuring cell (to the left of the thermostat).

The measuring cell holding the crystal and fluid sample was contained in a pressure vessel rated to $93 \mathrm{MPa}$. This, in turn, was held in a thermostated liquid bath operating from $-45^{\circ} \mathrm{C}$ to $150{ }^{\circ} \mathrm{C}$. A photo of the bath and associated fluid-handling manifold is shown as Figure A.1-4. A photo of the instruments (which are located in the adjacent room) is shown as Figure A.1-5. A schematic of the measuring cell is shown as Figure A.1-6.

The temperature of the fluid bath was measured with a long-stem 25-ohm standard platinum resistance thermometer (SPRT); the temperature-sensing portion of the SPRT was located immediately adjacent to the pressure vessel, as indicated in Figure A.1-6. The resistance of the SPRT was ratioed to a standard resistor with an AC resistance bridge. The pressure was measured with a vibrating-quartz-crystal pressure transducer with a maximum pressure of $138 \mathrm{MPa}$. The transducer was held at room temperature. 


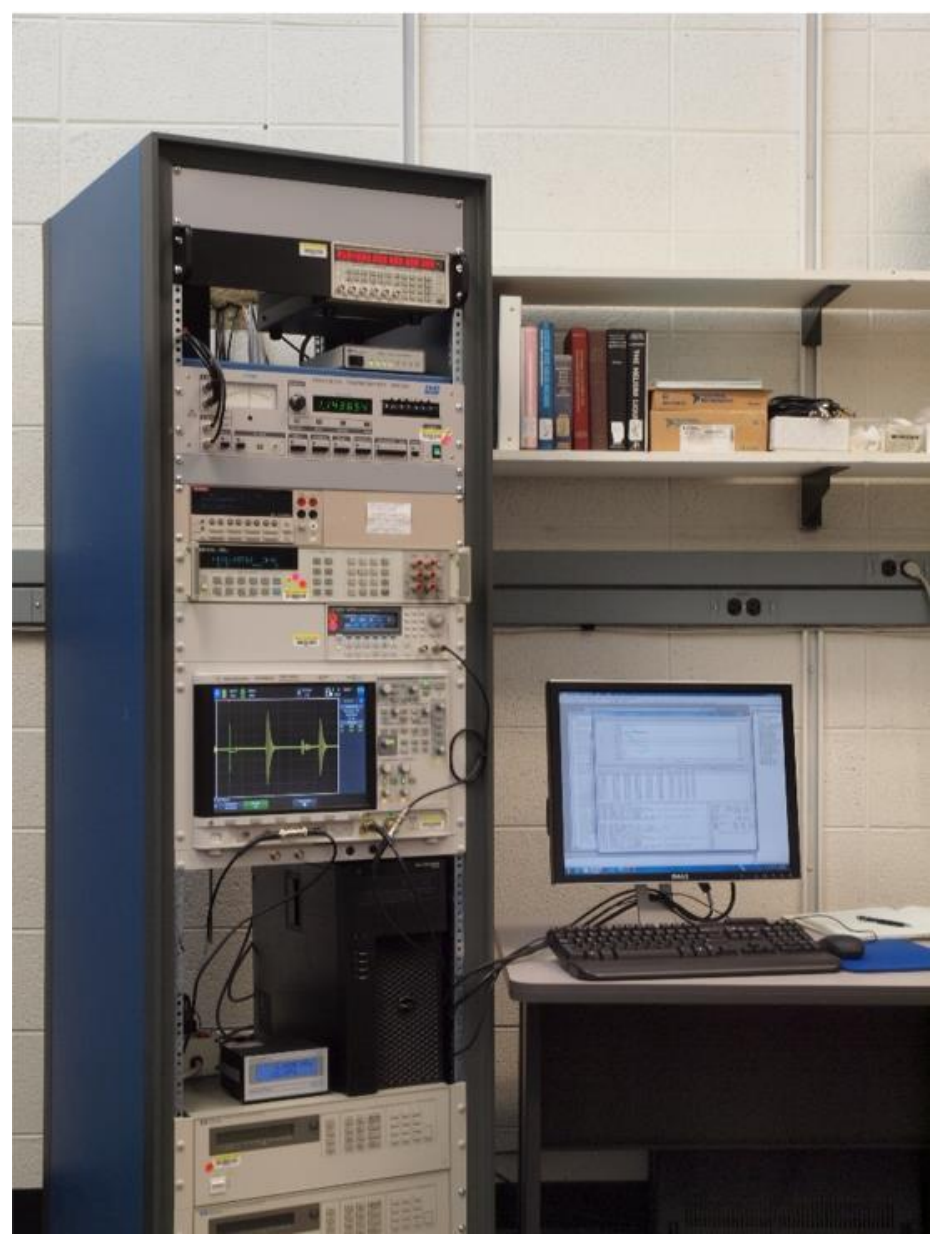

Figure A.1-5. Instrument rack for the pulse-echo speed of sound instrument.

Speed of sound measurement sequence. The entire experiment was controlled by a PC running a custom control program. At each $(T, P)$ state point multiple echo signals were recorded and analyzed. The pressure of the fluid sample and the temperature of the thermostat bath were scanned every $30 \mathrm{~s}$. The approach to equilibrium conditions was determined by monitoring three quantities: (1) the difference of the average temperature computed over the previous eight scans compared to the set-point temperature; (2) the standard deviation of the previous eight temperature scans; (3) the rate of change of pressure with time, computed with a linear fit of the previous eight pressure readings. When all three of these were within preset tolerances a "converged" flag was set in the control program, and measurements commenced following an additional equilibration time of 20 minutes.

A single measurement set comprised recording three echo signals and the four temperature and pressure readings made at the start and end of the set and between the recording of the echoes. Four such sets, spaced 10 minutes apart, were recorded before moving to the next ( $T$, $P)$ state point. These raw data were analyzed with a separate program to generate the $(T, P$, w) data points. 


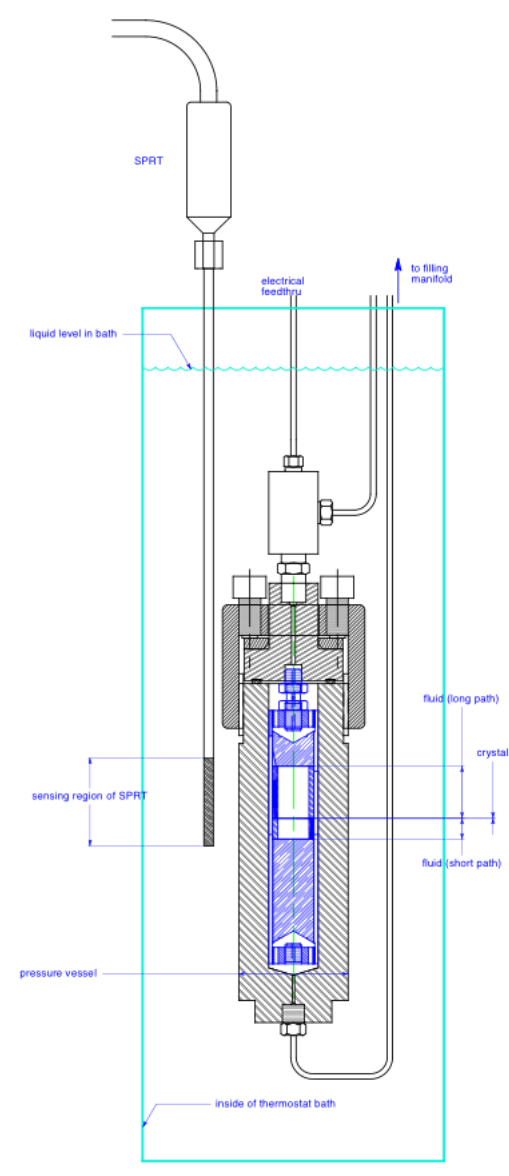

Figure A.1-6. Schematic diagram of the measuring cell inside the pressure vessel.

When measurements at the first temperature were completed, the temperature was increased by an increment of $5 \mathrm{~K}$ or $10 \mathrm{~K}$; since the cell was completely filled with liquid, the increase in temperature also increased the pressure. Measurements continued along this pseudoisochore (line of nearly constant density) until either the desired maximum temperature or maximum pressure was reached. The bath was then cooled to the starting temperature of the next isochore, and a portion of the sample in the measuring cell was vented to a waste bottle to achieve a starting pressure for the next isochore of $1 \mathrm{MPa}$ or the saturation pressure (whichever was greater). The next isochore then commenced. This process was repeated to cover the liquid surface.

Speed of sound calibrations and measurement uncertainties. The difference of the path lengths in the measuring cell, i.e., $\left(L_{2}-L_{1}\right)$ in Eq. (A.1-4), was calibrated as a function of temperature and pressure with measurements on propane over the temperature range of $260 \mathrm{~K}$ to $420 \mathrm{~K}$, with pressures to $56 \mathrm{MPa}$. The propane speed of sound, as calculated with the equation of state (EOS) of Lemmon et al. ${ }^{80}$, was taken as the known quantity in the calibration. The EOS, in turn, represents the high-accuracy propane measurements of Meier and Kabelac ${ }^{81}$ with an average absolute deviation of $0.012 \%$. The RMS deviation of the calibration equation from the measurements was $0.010 \%$. Combining, in quadrature, the deviation of the EOS from the data of Meier and Kabelac ${ }^{81}$ with the deviation of the path- 
length calibration equation from the present measurements yields an estimated combined standard uncertainty of $0.016 \%$ in our speed of sound measurements.

The SPRT, standard resistor, and resistance bridge were calibrated as a system over the range of $234.316 \mathrm{~K}$ to $429.749 \mathrm{~K}\left(-38.834{ }^{\circ} \mathrm{C}\right.$ to $156.599{ }^{\circ} \mathrm{C}$ ) with fixed-point cells (mercury triple point, water triple point, and indium freezing point). The standard uncertainty in the SPRT/resistor/bridge system was estimated as $3 \mathrm{mK}$. The short-term (minute-to-minute) variations in the fluid-bath temperature were $2 \mathrm{mK}$ or less. No long-term (hour-to-hour) variation was observed. The temperature gradients in the bath were less than $2.5 \mathrm{mK}$ over the region of the pressure vessel. The combined standard $(k=1)$ uncertainty in the temperature measurement, including the effects of the SPRT, standard resistor, resistance bridge, calibration standards, stability of the fluid bath, and temperature gradients in the bath was $4 \mathrm{mK}$.

The pressure transducer was calibrated by the manufacturer with piston gages; this calibration included a temperature-compensation term. The zero of the transducer was checked regularly (while the system was evacuated between samples) and readings were corrected for any drift in the zero. The standard uncertainty in pressure was 0.007 MPa.

When reporting the uncertainties in experimental data it is customary to combine the effects of the state-point uncertainty (i.e., the effects of the uncertainties in temperature, pressure, and composition) with those in the uncertainty of the primary measurand (i.e., the speed of sound):

$$
U_{c}(w)=2 \times 100 \times\left\{u^{2}(w)+\left[\frac{\partial w}{\partial T}\right]^{2} u^{2}(T)+\left[\frac{\partial w}{\partial p}\right]^{2} u^{2}(P)\right\} / w,
$$

where the $u(x)$ are the standard $(k=1)$ uncertainties in the different measurands (temperature, pressure, and speed of sound), the derivatives of the speed of sound with temperature and pressure are computed with an equation of state. The coverage factor of 2 corresponds to a $95 \%$ confidence interval, and the factor of 100 converts the relative deviation to a percentage deviation. The $U_{c}(w)$ is the relative, combined, expanded $(k=2)$ uncertainty in the speed of sound; it averaged $0.07 \%$ for the present measurements.

Speed of sound results. The measurements carried out are depicted in Figure 3.1-2. The measured data, as well as the relative, combined, expanded $(k=2)$ uncertainty in the speed of sound for each point, are reported in Tables A.1-15 through A.1-20. As indicated above, the data in these tables are the averages of four sets of three replicates each. 
Table A.1-15. Measured speed of sound data for the system R-1234yf/134a at a molar composition of $(0.33634 / 0.66366)$.

Listed are temperature $T$, pressure $P$, speed of sound $w$, relative combined, expanded $(k=2)$ state point uncertainty in the speed of sound $U_{c}$, and relative deviation from the mixture EOS. Average values for the replicate measurements at each $(T, P)$ state point are given. The different isochores are separated by blank lines.

$\begin{array}{crrrr}T / \mathrm{K} & P / \mathrm{MPa} & w / \mathrm{m} \cdot \mathrm{s}^{-1} & U_{\mathrm{c}} / \% & \Delta_{w} / \% \\ & & & & \\ 230.013 & 0.353 & 790.84 & 0.041 & 0.126 \\ 235.007 & 0.658 & 769.59 & 0.041 & 0.164 \\ 240.004 & 0.678 & 746.67 & 0.042 & 0.192 \\ 245.004 & 0.690 & 723.74 & 0.044 & 0.209 \\ 250.005 & 0.704 & 700.89 & 0.046 & 0.217 \\ 254.995 & 1.231 & 682.11 & 0.047 & 0.227 \\ 259.987 & 5.247 & 689.09 & 0.045 & 0.131 \\ 264.996 & 9.178 & 695.40 & 0.044 & 0.055 \\ & & & & \\ 254.993 & 0.651 & 677.79 & 0.048 & 0.240 \\ 259.986 & 4.420 & 683.24 & 0.046 & 0.150 \\ 264.996 & 8.392 & 690.07 & 0.045 & 0.067 \\ 269.993 & 12.399 & 697.15 & 0.043 & -0.002 \\ & & & & \\ 259.986 & 1.278 & 659.86 & 0.049 & 0.226 \\ 264.995 & 5.122 & 666.92 & 0.047 & 0.130 \\ 269.993 & 9.068 & 674.67 & 0.046 & 0.049 \\ 274.996 & 12.964 & 681.96 & 0.044 & -0.012 \\ 2796 & & & & \\ 264.994 & 1.260 & 637.06 & 0.052 & 0.224 \\ 269.991 & 4.977 & 644.66 & 0.050 & 0.128 \\ 274.995 & 8.710 & 652.21 & 0.048 & 0.049 \\ 279.999 & 12.442 & 659.66 & 0.046 & -0.014 \\ 269.991 & 1.167 & 613.65 & 0.055 & 0.223 \\ 274.995 & 4.716 & 621.41 & 0.053 & 0.131 \\ 280.000 & 8.276 & 629.11 & 0.050 & 0.054 \\ 285.001 & 11.853 & 636.78 & 0.048 & -0.011 \\ & & & & \\ 274.993 & 1.193 & 591.14 & 0.059 & 0.215 \\ & 4.586 & 599.13 & 0.056 & 0.129 \\ & 7.981 & 606.95 & 0.053 & 0.056 \\ & 11.378 & 614.61 & 0.051 & -0.003\end{array}$


Table A.1-15. (continued)

$\begin{array}{rrrrr}T / \mathrm{K} & P / \mathrm{MPa} & w / \mathrm{m} \cdot \mathrm{s}^{-1} & U_{\mathrm{c}} / \% & \Delta_{w} / \% \\ & & & & \\ 279.998 & 0.890 & 565.36 & 0.064 & 0.212 \\ 285.000 & 4.124 & 573.74 & 0.060 & 0.131 \\ 290.003 & 7.359 & 581.87 & 0.057 & 0.066 \\ 294.998 & 10.590 & 589.77 & 0.054 & 0.012 \\ & & & & \\ 284.998 & 1.183 & 545.28 & 0.068 & 0.198 \\ 290.001 & 4.259 & 553.65 & 0.064 & 0.126 \\ 294.997 & 7.347 & 561.90 & 0.060 & 0.068 \\ 299.998 & 10.440 & 569.91 & 0.057 & 0.019 \\ & & & & \\ 290.000 & 1.199 & 522.32 & 0.074 & 0.186 \\ 294.997 & 4.134 & 531.05 & 0.069 & 0.126 \\ 299.999 & 7.087 & 539.59 & 0.065 & 0.077 \\ 304.998 & 10.041 & 547.87 & 0.061 & 0.035 \\ & & & & \\ 294.996 & 1.213 & 499.13 & 0.081 & 0.170 \\ 299.998 & 4.004 & 508.17 & 0.075 & 0.134 \\ 304.998 & 6.801 & 516.85 & 0.070 & 0.095 \\ 310.000 & 9.603 & 525.24 & 0.066 & 0.059 \\ & & & & \\ 299.997 & 1.313 & 476.73 & 0.089 & 0.158 \\ 304.996 & 3.957 & 485.96 & 0.082 & 0.136 \\ 309.999 & 6.613 & 494.84 & 0.076 & 0.110 \\ 314.995 & 9.266 & 503.35 & 0.071 & 0.084 \\ & & & & \\ 304.995 & 1.397 & 453.89 & 0.099 & 0.136 \\ 309.998 & 3.908 & 463.47 & 0.091 & 0.134 \\ 314.995 & 6.420 & 472.55 & 0.084 & 0.123 \\ 320.004 & 8.941 & 481.25 & 0.078 & 0.106 \\ & & & & \\ 309.997 & 1.503 & 431.03 & 0.111 & 0.103 \\ 314.994 & 3.859 & 440.71 & 0.101 & 0.128 \\ 320.004 & 6.236 & 450.01 & 0.092 & 0.136 \\ 325.009 & 8.608 & 458.78 & 0.085 & 0.131 \\ & & & & \\ 314.992 & 1.733 & 409.82 & 0.124 & 0.062 \\ 320.002 & 3.960 & 419.66 & 0.112 & 0.109 \\ 325.008 & 6.201 & 429.07 & 0.102 & 0.136 \\ 330.000 & 8.441 & 437.99 & 0.094 & 0.148 \\ & & & & \end{array}$


Table A.1-15. (continued)

$\begin{array}{ccccc}T / \mathrm{K} & P / \mathrm{MPa} & w / \mathrm{m} \cdot \mathrm{s}^{-1} & U_{\mathrm{c}} / \% & \Delta_{w} / \% \\ & & & & \\ 320.002 & 1.827 & 386.04 & 0.142 & -0.020 \\ 325.008 & 3.924 & 396.35 & 0.127 & 0.078 \\ 330.000 & 6.023 & 405.99 & 0.115 & 0.136 \\ 335.004 & 8.112 & 414.85 & 0.105 & 0.170 \\ & & & & \\ 325.006 & 2.025 & 363.70 & 0.164 & -0.098 \\ 329.999 & 3.980 & 374.21 & 0.145 & 0.055 \\ 335.002 & 5.935 & 383.73 & 0.130 & 0.136 \\ 340.007 & 7.905 & 392.88 & 0.118 & 0.188 \\ & & & & \\ 329.998 & 2.229 & 341.01 & 0.191 & -0.240 \\ 335.002 & 4.041 & 351.53 & 0.167 & -0.017 \\ 340.007 & 5.877 & 361.47 & 0.148 & 0.113 \\ 345.013 & 7.718 & 370.79 & 0.134 & 0.203 \\ & & & & \\ 335.001 & 2.392 & 317.01 & 0.230 & -0.333 \\ 340.006 & 4.087 & 328.15 & 0.198 & -0.056 \\ 345.013 & 5.792 & 338.36 & 0.173 & 0.121 \\ 340.006 & 2.607 & 293.45 & 0.282 & -0.412 \\ 345.012 & 4.167 & 304.49 & 0.237 & -0.194 \\ 345.012 & 2.910 & 271.59 & 0.345 & -0.484\end{array}$


Table A.1-16. Measured speed of sound data for the system R-1234yf/134a at a molar composition of $(0.64709 / 0.35291)$.

Listed are temperature $T$, pressure $P$, speed of sound $w$, relative combined, expanded $(k=2)$ state point uncertainty in the speed of sound $U_{c}$, and relative deviation from the mixture EOS. Average values for the replicate measurements at each $(T, P)$ state point are given. The different isochores are separated by blank lines.

$\begin{array}{crccc}T / \mathrm{K} & P / \mathrm{MPa} & w / \mathrm{m} \cdot \mathrm{s}^{-1} & U_{\mathrm{c}} / \% & \Delta_{w} / \% \\ & & & & \\ 235.007 & 0.663 & 746.03 & 0.043 & -0.064 \\ 240.002 & 0.672 & 723.28 & 0.044 & -0.042 \\ 245.003 & 0.694 & 700.73 & 0.046 & -0.030 \\ 250.004 & 0.708 & 678.16 & 0.048 & -0.037 \\ 254.995 & 0.720 & 655.72 & 0.050 & -0.050 \\ 259.987 & 2.996 & 651.70 & 0.050 & -0.095 \\ 264.997 & 6.626 & 658.38 & 0.048 & -0.177 \\ 269.993 & 10.288 & 665.31 & 0.046 & -0.244 \\ & & & & \\ 260.001 & 0.801 & 633.95 & 0.053 & -0.054 \\ 264.995 & 4.338 & 640.85 & 0.051 & -0.142 \\ 269.993 & 7.925 & 648.05 & 0.049 & -0.215 \\ 274.995 & 11.487 & 654.98 & 0.047 & -0.273 \\ & & & & \\ 264.994 & 1.250 & 615.38 & 0.055 & -0.093 \\ 269.992 & 4.705 & 622.85 & 0.053 & -0.174 \\ 274.995 & 8.164 & 630.22 & 0.050 & -0.237 \\ 280.000 & 11.622 & 637.48 & 0.049 & -0.286 \\ 2799 & & & \\ 269.990 & 1.132 & 591.90 & 0.059 & -0.145 \\ 274.995 & 4.413 & 599.48 & 0.056 & -0.205 \\ 279.999 & 7.714 & 607.04 & 0.053 & -0.258 \\ 285.001 & 11.008 & 614.44 & 0.051 & -0.297 \\ 274.994 & 1.218 & 570.34 & 0.062 & -0.178 \\ 279.999 & 4.366 & 578.19 & 0.059 & -0.232 \\ 285.001 & 7.517 & 585.89 & 0.056 & -0.273 \\ 290.002 & 10.656 & 593.38 & 0.054 & -0.296 \\ & & & & \\ 279.998 & 1.243 & 548.15 & 0.067 & -0.220 \\ & 4.239 & 556.18 & 0.063 & -0.254 \\ & 10.251 & 571.77 & 0.057 & -0.298\end{array}$


Table A.1-16. (continued)

$\begin{array}{ccccc}T / \mathrm{K} & P / \mathrm{MPa} & w / \mathrm{m}^{-1} \mathrm{~s}^{-1} & U_{\mathrm{c}} / \% & \Delta_{w} / \% \\ & & & & \\ 284.998 & 1.205 & 525.15 & 0.073 & -0.271 \\ 290.002 & 4.071 & 533.56 & 0.068 & -0.283 \\ 294.998 & 6.933 & 541.67 & 0.064 & -0.293 \\ 299.998 & 9.802 & 549.61 & 0.061 & -0.298 \\ & & & & \\ 290.000 & 1.169 & 501.82 & 0.080 & -0.348 \\ 294.997 & 3.881 & 510.45 & 0.074 & -0.328 \\ 299.999 & 6.601 & 518.81 & 0.070 & -0.309 \\ 304.997 & 9.317 & 526.84 & 0.066 & -0.297 \\ & & & & \\ 294.995 & 1.281 & 480.27 & 0.087 & -0.381 \\ 299.998 & 3.860 & 489.10 & 0.081 & -0.338 \\ 304.998 & 6.446 & 497.61 & 0.075 & -0.309 \\ 309.999 & 9.038 & 505.86 & 0.071 & -0.287\end{array}$


Table A.1-17. Measured speed of sound data for the system R-134a/1234ze(E) at a molar composition of $(0.32916 / 0.67084)$.

Listed are temperature $T$, pressure $P$, speed of sound $w$, relative combined, expanded $(k=2)$ state point uncertainty in the speed of sound $U_{c}$, and relative deviation from the mixture EOS. Average values for the replicate measurements at each $(T, P)$ state point are given.

$\begin{array}{crccc}T / \mathrm{K} & P / \mathrm{MPa} & w / \mathrm{m} \cdot \mathrm{s}^{-1} & U_{\mathrm{c}} / \% & \Delta_{w} / \% \\ & & & & \\ 235.008 & 0.525 & 812.57 & 0.039 & 1.043 \\ 240.003 & 0.537 & 789.62 & 0.040 & 1.009 \\ 245.004 & 0.541 & 766.62 & 0.041 & 0.966 \\ 250.004 & 0.549 & 743.64 & 0.042 & 0.915 \\ 254.994 & 0.553 & 720.81 & 0.044 & 0.875 \\ 259.987 & 0.559 & 698.02 & 0.046 & 0.838 \\ 269.992 & 1.140 & 657.06 & 0.050 & 0.783 \\ 274.995 & 4.782 & 663.40 & 0.048 & 0.710 \\ 280.000 & 8.458 & 669.98 & 0.046 & 0.634 \\ 285.001 & 12.144 & 676.62 & 0.045 & 0.561 \\ 290.003 & 15.823 & 683.20 & 0.044 & 0.493 \\ 294.998 & 19.487 & 689.68 & 0.043 & 0.430 \\ 299.999 & 23.144 & 696.07 & 0.042 & 0.373 \\ 304.997 & 26.739 & 702.09 & 0.041 & 0.324 \\ 310.000 & 30.316 & 707.98 & 0.040 & 0.278 \\ 314.994 & 33.894 & 713.91 & 0.040 & 0.238 \\ 320.004 & 37.460 & 719.74 & 0.039 & 0.203 \\ 325.010 & 41.009 & 725.47 & 0.038 & 0.171 \\ 330.000 & 44.515 & 731.03 & 0.038 & 0.144 \\ 335.004 & 47.984 & 736.38 & 0.038 & 0.119 \\ 340.009 & 51.440 & 741.68 & 0.037 & 0.098 \\ 274.989 & 1.010 & 633.32 & 0.052 & 0.749 \\ 279.995 & 4.674 & 641.29 & 0.050 & 0.663 \\ 284.997 & 8.321 & 649.02 & 0.048 & 0.584 \\ 290.000 & 11.947 & 656.43 & 0.047 & 0.506 \\ 294.995 & 15.574 & 663.80 & 0.045 & 0.436 \\ 299.996 & 19.173 & 670.85 & 0.044 & 0.374 \\ 304.995 & 22.769 & 677.82 & 0.043 & 0.317 \\ 309.998 & 26.350 & 684.61 & 0.042 & 0.268 \\ 314.993 & 29.899 & 691.17 & 0.041 & 0.224 \\ 320.005 & 33.452 & 697.64 & 0.040 & 0.183 \\ 325.008 & 36.980 & 703.96 & 0.040 & 0.148 \\ 329.999 & 40.468 & 710.07 & 0.039 & 0.119\end{array}$


Table A.1-17. (continued)

$\begin{array}{crrrr}T / \mathrm{K} & P / \mathrm{MPa} & w / \mathrm{m} \cdot \mathrm{s}^{-1} & U_{\mathrm{c}} / \% & \Delta_{w} / \% \\ & & & & \\ 335.004 & 43.903 & 715.84 & 0.039 & 0.092 \\ 340.008 & 47.327 & 721.56 & 0.038 & 0.071 \\ 345.014 & 50.738 & 727.19 & 0.038 & 0.052 \\ & & & & \\ 279.994 & 0.983 & 610.28 & 0.056 & 0.712 \\ 284.996 & 4.446 & 618.17 & 0.053 & 0.622 \\ 289.998 & 7.896 & 625.79 & 0.051 & 0.536 \\ 294.994 & 11.388 & 633.58 & 0.049 & 0.451 \\ 299.996 & 14.861 & 641.10 & 0.047 & 0.380 \\ 304.995 & 18.318 & 648.38 & 0.046 & 0.316 \\ 309.997 & 21.756 & 655.44 & 0.045 & 0.261 \\ 314.993 & 25.181 & 662.33 & 0.043 & 0.211 \\ 320.003 & 28.593 & 669.03 & 0.043 & 0.169 \\ 325.009 & 31.978 & 675.51 & 0.042 & 0.130 \\ 330.000 & 35.346 & 681.89 & 0.041 & 0.098 \\ 335.003 & 38.647 & 687.82 & 0.040 & 0.070 \\ 340.008 & 41.950 & 693.72 & 0.040 & 0.043 \\ 345.012 & 45.243 & 699.54 & 0.039 & 0.020 \\ & & & & \\ 284.995 & 1.030 & 587.84 & 0.060 & 0.670 \\ 289.997 & 4.364 & 596.15 & 0.056 & 0.572 \\ 294.994 & 7.702 & 604.29 & 0.054 & 0.484 \\ 299.996 & 11.081 & 612.50 & 0.052 & 0.398 \\ 304.995 & 14.423 & 620.29 & 0.050 & 0.330 \\ 309.997 & 17.750 & 627.78 & 0.048 & 0.265 \\ 314.992 & 21.047 & 634.96 & 0.046 & 0.212 \\ 320.001 & 24.301 & 641.73 & 0.045 & 0.169 \\ 325.008 & 27.528 & 648.23 & 0.044 & 0.124 \\ 329.999 & 30.759 & 654.73 & 0.043 & 0.086 \\ 335.003 & 33.970 & 661.02 & 0.042 & 0.051 \\ 340.008 & 37.171 & 667.20 & 0.041 & 0.021 \\ 345.013 & 40.356 & 673.25 & 0.041 & -0.004 \\ & & & & \\ 289.996 & 1.161 & 566.13 & 0.064 & 0.612 \\ 294.992 & 4.345 & 574.65 & 0.060 & 0.515 \\ 299.994 & 7.543 & 583.01 & 0.057 & 0.428 \\ 304.994 & 10.731 & 591.05 & 0.054 & 0.351 \\ 309.996 & 13.911 & 598.82 & 0.052 & 0.282 \\ 314.992 & 17.077 & 606.34 & 0.050 & 0.222 \\ & & & & \end{array}$


Table A.1-17. (continued)

$\begin{array}{rrrrr}T / \mathrm{K} & P / \mathrm{MPa} & w / \mathrm{m} \cdot \mathrm{s}^{-1} & U_{\mathrm{c}} / \% & \Delta_{w} / \% \\ & & & & \\ 320.003 & 20.248 & 613.69 & 0.049 & 0.168 \\ 325.008 & 23.442 & 621.11 & 0.047 & 0.122 \\ 329.999 & 26.574 & 628.01 & 0.046 & 0.080 \\ 335.003 & 29.665 & 634.47 & 0.045 & 0.036 \\ 340.006 & 32.755 & 640.89 & 0.044 & 0.000 \\ 345.014 & 35.827 & 647.15 & 0.043 & -0.026 \\ & & & & \\ 294.991 & 0.899 & 540.26 & 0.070 & 0.549 \\ 299.994 & 3.918 & 549.04 & 0.065 & 0.460 \\ 304.993 & 6.942 & 557.56 & 0.062 & 0.380 \\ 309.996 & 9.974 & 565.85 & 0.059 & 0.306 \\ 314.991 & 12.986 & 573.78 & 0.056 & 0.244 \\ 320.001 & 15.983 & 581.33 & 0.054 & 0.188 \\ 325.007 & 18.972 & 588.66 & 0.052 & 0.135 \\ 329.999 & 21.942 & 595.75 & 0.050 & 0.086 \\ 335.003 & 24.877 & 602.46 & 0.048 & 0.044 \\ 340.007 & 27.825 & 609.15 & 0.047 & 0.004 \\ 345.013 & 30.764 & 615.69 & 0.046 & -0.031 \\ & & & & \\ 299.991 & 0.756 & 515.11 & 0.077 & 0.473 \\ 304.993 & 3.626 & 524.27 & 0.072 & 0.399 \\ 309.995 & 6.506 & 533.14 & 0.067 & 0.331 \\ 314.991 & 9.373 & 541.58 & 0.063 & 0.270 \\ 320.001 & 12.244 & 549.72 & 0.060 & 0.211 \\ 325.007 & 15.100 & 557.52 & 0.057 & 0.159 \\ 329.998 & 17.941 & 565.04 & 0.055 & 0.108 \\ 335.002 & 20.767 & 572.22 & 0.053 & 0.059 \\ 340.008 & 23.590 & 579.25 & 0.051 & 0.014 \\ 345.013 & 26.400 & 586.06 & 0.049 & -0.027 \\ & & & & \\ 304.991 & 1.377 & 498.72 & 0.081 & 0.388 \\ 309.994 & 4.125 & 507.93 & 0.076 & 0.335 \\ 314.990 & 6.876 & 516.79 & 0.071 & 0.285 \\ 320.001 & 9.632 & 525.29 & 0.067 & 0.233 \\ 325.007 & 12.380 & 533.43 & 0.063 & 0.181 \\ 329.998 & 15.115 & 541.25 & 0.060 & 0.131 \\ 335.003 & 17.824 & 548.60 & 0.057 & 0.079 \\ 340.008 & 20.519 & 555.69 & 0.055 & 0.034 \\ 345.013 & 23.229 & 562.73 & 0.053 & -0.014 \\ & & & & \end{array}$


Table A.1-17. (continued)

$\begin{array}{rrrrr}T / \mathrm{K} & p / \mathrm{MPa} & w / \mathrm{m} \cdot \mathrm{s}^{-1} & U_{\mathrm{c}} \% & \Delta_{w} / \% \\ 309.993 & 1.253 & 473.32 & 0.091 & 0.296 \\ 314.989 & 3.848 & 482.89 & 0.084 & 0.278 \\ 320.000 & 6.456 & 492.03 & 0.078 & 0.249 \\ 325.006 & 9.061 & 500.75 & 0.073 & 0.210 \\ 329.997 & 11.654 & 509.07 & 0.069 & 0.166 \\ 335.002 & 14.232 & 516.91 & 0.065 & 0.118 \\ 340.007 & 16.806 & 524.47 & 0.062 & 0.068 \\ 345.012 & 19.379 & 531.84 & 0.059 & 0.018 \\ 314.990 & 1.546 & 453.04 & 0.100 & 0.206 \\ 319.999 & 4.024 & 462.90 & 0.092 & 0.223 \\ 325.005 & 6.490 & 472.10 & 0.085 & 0.222 \\ 329.997 & 8.953 & 480.84 & 0.079 & 0.197 \\ 335.002 & 11.402 & 489.00 & 0.074 & 0.153 \\ 340.008 & 13.858 & 496.96 & 0.070 & 0.106 \\ 345.013 & 16.312 & 504.64 & 0.066 & 0.055 \\ & & & & \\ 319.999 & 1.756 & 431.60 & 0.111 & 0.132 \\ 325.005 & 4.078 & 441.42 & 0.101 & 0.196 \\ 329.997 & 6.412 & 450.80 & 0.093 & 0.209 \\ 335.001 & 8.742 & 459.60 & 0.087 & 0.191 \\ 340.007 & 11.076 & 468.04 & 0.081 & 0.155 \\ 345.013 & 13.404 & 476.10 & 0.076 & 0.108 \\ 325.005 & 1.948 & 409.78 & 0.125 & 0.079 \\ 329.997 & 4.146 & 419.95 & 0.113 & 0.176 \\ 335.001 & 6.350 & 429.46 & 0.104 & 0.206 \\ 340.007 & 8.553 & 438.42 & 0.095 & 0.199 \\ 345.012 & 10.760 & 446.98 & 0.089 & 0.163 \\ 329.998 & 2.044 & 386.06 & 0.144 & 0.020 \\ 335.002 & 4.096 & 396.26 & 0.129 & 0.145 \\ 340.006 & 6.157 & 405.92 & 0.117 & 0.205 \\ 345.013 & 8.225 & 415.07 & 0.107 & 0.205 \\ 335.000 & 2.385 & 366.39 & 0.163 & 0.003 \\ 340.006 & 4.329 & 377.01 & 0.145 & 0.176 \\ 345.013 & 6.278 & 386.87 & 0.130 & 0.244 \\ & & & & \\ 340.005 & 2.348 & 338.90 & 0.199 & -0.022 \\ & 4.140 & 349.93 & 0.174 & 0.211 \\ & 2.550 & 315.54 & 0.238 & 0.004\end{array}$


Table A.1-18. Measured speed of sound data for the system R-134a/1234ze(E) at a molar composition of $(0.63688 / 36312)$.

Listed are temperature $T$, pressure $P$, speed of sound $w$, relative combined, expanded $(k=2)$ state point uncertainty in the speed of sound $U_{c}$, and relative deviation from the mixture EOS. Average values for the replicate measurements at each $(T, P)$ state point are given. The different isochores are separated by blank lines.

$\begin{array}{ccccc}T / \mathrm{K} & P / \mathrm{MPa} & w / \mathrm{m}^{-1} & U_{\mathrm{c}} / \% & \Delta_{w} / \% \\ 229.997 & 0.131 & 829.10 & 0.039 & 1.135 \\ 235.007 & 0.589 & 808.34 & 0.039 & 1.121 \\ 240.004 & 0.609 & 785.19 & 0.040 & 1.098 \\ 245.006 & 0.605 & 761.97 & 0.041 & 1.077 \\ 250.005 & 0.609 & 738.83 & 0.043 & 1.048 \\ 254.994 & 0.618 & 715.85 & 0.044 & 1.025 \\ 259.988 & 0.611 & 692.86 & 0.046 & 1.017 \\ 269.993 & 5.812 & 685.99 & 0.045 & 0.853 \\ 274.995 & 9.842 & 693.24 & 0.044 & 0.748 \\ 280.000 & 13.884 & 700.47 & 0.043 & 0.653 \\ 285.001 & 17.913 & 707.56 & 0.042 & 0.568 \\ 290.003 & 21.919 & 714.46 & 0.041 & 0.492 \\ 294.998 & 25.905 & 721.19 & 0.040 & 0.423 \\ 299.999 & 29.876 & 727.77 & 0.039 & 0.361 \\ 304.998 & 33.823 & 734.20 & 0.039 & 0.306 \\ 310.000 & 37.736 & 740.43 & 0.038 & 0.257 \\ 314.995 & 41.622 & 746.51 & 0.038 & 0.211 \\ 320.004 & 45.494 & 752.47 & 0.037 & 0.169 \\ 269.993 & 3.174 & 666.80 & 0.048 & 0.924 \\ 274.995 & 7.130 & 674.44 & 0.046 & 0.803 \\ 280.000 & 11.085 & 681.95 & 0.045 & 0.695 \\ 285.001 & 15.028 & 689.29 & 0.043 & 0.600 \\ 290.003 & 18.959 & 696.46 & 0.042 & 0.514 \\ 299.999 & 26.745 & 710.16 & 0.040 & 0.372 \\ 304.998 & 30.611 & 716.76 & 0.040 & 0.311 \\ 310.000 & 34.457 & 723.21 & 0.039 & 0.257 \\ 314.993 & 38.265 & 729.45 & 0.039 & 0.210 \\ 320.004 & 42.025 & 735.38 & 0.038 & 0.167 \\ 325.008 & 45.773 & 741.24 & 0.038 & 0.125\end{array}$


Table A.1-18. (continued)

$\begin{array}{rrrrr}T / \mathrm{K} & P / \mathrm{MPa} & w / \mathrm{m} \cdot \mathrm{s}^{-1} & U_{\mathrm{c}} / \% & \Delta_{w} / \% \\ & & & & \\ 269.987 & 1.099 & 650.78 & 0.050 & 0.987 \\ 274.991 & 4.970 & 658.61 & 0.048 & 0.854 \\ 279.996 & 8.848 & 666.34 & 0.046 & 0.736 \\ 284.998 & 12.716 & 673.89 & 0.045 & 0.632 \\ 290.001 & 16.573 & 681.26 & 0.044 & 0.540 \\ 294.996 & 20.399 & 688.35 & 0.043 & 0.458 \\ 299.998 & 24.212 & 695.26 & 0.042 & 0.386 \\ 304.997 & 28.005 & 702.00 & 0.041 & 0.321 \\ 309.999 & 31.782 & 708.59 & 0.040 & 0.263 \\ 314.996 & 35.538 & 715.02 & 0.039 & 0.211 \\ 320.004 & 39.328 & 721.55 & 0.039 & 0.164 \\ 325.009 & 43.044 & 727.69 & 0.038 & 0.123 \\ 330.000 & 46.700 & 733.54 & 0.038 & 0.086 \\ 335.004 & 50.316 & 739.13 & 0.037 & 0.050 \\ & & & & \\ 274.990 & 1.038 & 627.36 & 0.053 & 0.977 \\ 279.995 & 4.733 & 635.34 & 0.051 & 0.836 \\ 284.997 & 8.434 & 643.22 & 0.049 & 0.713 \\ 290.000 & 12.131 & 650.91 & 0.047 & 0.604 \\ 294.995 & 15.817 & 658.41 & 0.046 & 0.507 \\ 299.997 & 19.484 & 665.63 & 0.044 & 0.423 \\ 304.995 & 23.137 & 672.69 & 0.043 & 0.349 \\ 309.998 & 26.772 & 679.54 & 0.042 & 0.283 \\ 314.994 & 30.385 & 686.21 & 0.041 & 0.225 \\ 320.004 & 33.986 & 692.71 & 0.040 & 0.173 \\ 325.009 & 37.562 & 699.04 & 0.040 & 0.126 \\ 330.000 & 41.101 & 705.15 & 0.039 & 0.083 \\ 335.003 & 44.595 & 710.97 & 0.039 & 0.044 \\ 340.007 & 48.088 & 716.76 & 0.038 & 0.008 \\ & & & & \\ 279.994 & 1.100 & 604.88 & 0.057 & 0.958 \\ 284.996 & 4.633 & 613.08 & 0.054 & 0.813 \\ 289.999 & 8.175 & 621.14 & 0.051 & 0.686 \\ 294.995 & 11.707 & 628.97 & 0.049 & 0.575 \\ 299.996 & 15.229 & 636.54 & 0.048 & 0.477 \\ 304.996 & 18.740 & 643.90 & 0.046 & 0.391 \\ 309.997 & 22.215 & 650.90 & 0.045 & 0.317 \\ 314.994 & 25.689 & 657.82 & 0.044 & 0.250\end{array}$


Table A.1-18. (continued)

$\begin{array}{rrrrr}T / \mathrm{K} & P / \mathrm{MPa} & w / \mathrm{m} \cdot \mathrm{s}^{-1} & U_{\mathrm{c}} / \% & \Delta_{w} / \% \\ & & & & \\ 320.003 & 29.154 & 664.55 & 0.043 & 0.190 \\ 325.009 & 32.599 & 671.10 & 0.042 & 0.137 \\ 330.000 & 36.014 & 677.47 & 0.041 & 0.090 \\ 335.004 & 39.380 & 683.47 & 0.040 & 0.046 \\ 340.008 & 42.752 & 689.47 & 0.040 & 0.007 \\ 345.013 & 46.114 & 695.38 & 0.039 & -0.028 \\ & & & & \\ 284.995 & 1.402 & 584.62 & 0.060 & 0.928 \\ 289.997 & 4.794 & 593.04 & 0.057 & 0.781 \\ 294.995 & 8.187 & 601.25 & 0.054 & 0.654 \\ 299.996 & 11.580 & 609.23 & 0.052 & 0.541 \\ 304.994 & 14.949 & 616.85 & 0.050 & 0.444 \\ 309.997 & 18.320 & 624.30 & 0.048 & 0.358 \\ 314.992 & 21.683 & 631.61 & 0.046 & 0.283 \\ 320.002 & 25.028 & 638.61 & 0.045 & 0.216 \\ 325.009 & 28.354 & 645.41 & 0.044 & 0.157 \\ 330.000 & 31.643 & 651.94 & 0.043 & 0.104 \\ 335.003 & 34.896 & 658.15 & 0.042 & 0.056 \\ 340.009 & 38.152 & 664.32 & 0.041 & 0.012 \\ 345.013 & 41.393 & 670.37 & 0.041 & -0.027 \\ & & & & \\ 289.996 & 1.173 & 559.27 & 0.065 & 0.917 \\ 294.993 & 4.392 & 567.96 & 0.061 & 0.768 \\ 299.994 & 7.623 & 576.43 & 0.058 & 0.638 \\ 304.994 & 10.850 & 584.61 & 0.055 & 0.525 \\ 309.996 & 14.070 & 592.50 & 0.053 & 0.426 \\ 314.993 & 17.273 & 600.08 & 0.051 & 0.339 \\ 320.002 & 20.478 & 607.48 & 0.049 & 0.262 \\ 325.008 & 23.661 & 614.60 & 0.047 & 0.194 \\ 330.000 & 26.822 & 621.49 & 0.046 & 0.133 \\ 335.002 & 29.939 & 627.95 & 0.045 & 0.077 \\ 340.007 & 33.106 & 634.64 & 0.044 & 0.025 \\ 345.012 & 36.236 & 641.04 & 0.043 & -0.019 \\ & & & & \\ 294.991 & 1.056 & 534.63 & 0.071 & 0.893 \\ 299.994 & 4.110 & 543.54 & 0.066 & 0.750 \\ 304.993 & 7.173 & 552.18 & 0.063 & 0.621 \\ 309.996 & 10.239 & 560.54 & 0.059 & 0.510 \\ 314.991 & 13.297 & 568.59 & 0.056 & 0.412 \\ & & & & \end{array}$


Table A.1-18. (continued)

$\begin{array}{rrrrr}T / \mathrm{K} & P / \mathrm{MPa} & w / \mathrm{m} \cdot \mathrm{s}^{-1} & U_{\mathrm{c}} / \% & \Delta_{w} / \% \\ & & & & \\ 320.003 & 16.348 & 576.32 & 0.054 & 0.327 \\ 325.008 & 19.388 & 583.79 & 0.052 & 0.249 \\ 329.997 & 22.381 & 590.80 & 0.050 & 0.178 \\ 335.002 & 25.368 & 597.59 & 0.048 & 0.114 \\ 340.009 & 28.359 & 604.32 & 0.047 & 0.058 \\ 345.012 & 31.341 & 610.89 & 0.046 & 0.006 \\ & & & & \\ 299.992 & 1.274 & 513.38 & 0.077 & 0.855 \\ 304.992 & 4.193 & 522.57 & 0.071 & 0.718 \\ 309.995 & 7.123 & 531.46 & 0.067 & 0.596 \\ 314.992 & 10.048 & 539.99 & 0.063 & 0.491 \\ 320.001 & 12.974 & 548.17 & 0.060 & 0.394 \\ 325.007 & 15.890 & 556.02 & 0.057 & 0.307 \\ 329.999 & 18.784 & 563.55 & 0.055 & 0.231 \\ 335.002 & 21.654 & 570.67 & 0.052 & 0.159 \\ 340.007 & 24.524 & 577.65 & 0.051 & 0.095 \\ 345.013 & 27.388 & 584.46 & 0.049 & 0.037 \\ & & & & \\ 304.992 & 1.802 & 495.84 & 0.082 & 0.803 \\ 309.995 & 4.602 & 505.21 & 0.076 & 0.679 \\ 314.990 & 7.403 & 514.17 & 0.071 & 0.567 \\ 320.001 & 10.211 & 522.77 & 0.067 & 0.466 \\ 325.007 & 13.007 & 530.95 & 0.063 & 0.375 \\ 329.998 & 15.788 & 538.80 & 0.060 & 0.291 \\ 335.002 & 18.543 & 546.15 & 0.057 & 0.210 \\ 340.008 & 21.304 & 553.38 & 0.055 & 0.139 \\ 345.013 & 24.059 & 560.42 & 0.053 & 0.074 \\ & & & & \\ 309.994 & 1.809 & 472.09 & 0.091 & 0.764 \\ 314.990 & 4.450 & 481.66 & 0.084 & 0.658 \\ 320.002 & 7.105 & 490.82 & 0.078 & 0.560 \\ 325.006 & 9.758 & 499.55 & 0.073 & 0.463 \\ 329.999 & 12.400 & 507.86 & 0.068 & 0.375 \\ 335.003 & 15.021 & 515.63 & 0.065 & 0.287 \\ 340.007 & 17.643 & 523.20 & 0.061 & 0.206 \\ 345.013 & 20.252 & 530.47 & 0.059 & 0.139 \\ & & & & \end{array}$


Table A.1-18. (continued)

$\begin{array}{crccc}T / \mathrm{K} & P / \mathrm{MPa} & w / \mathrm{m} \cdot \mathrm{s}^{-1} & U_{\mathrm{c}} / \% & \Delta_{w} / \% \\ & & & & \\ 314.990 & 1.964 & 449.98 & 0.101 & 0.715 \\ 319.999 & 4.471 & 459.83 & 0.092 & 0.637 \\ 325.006 & 6.981 & 469.12 & 0.085 & 0.552 \\ 329.998 & 9.484 & 477.92 & 0.079 & 0.464 \\ 335.003 & 11.973 & 486.12 & 0.074 & 0.371 \\ 340.007 & 14.470 & 494.12 & 0.070 & 0.285 \\ 345.013 & 16.965 & 501.84 & 0.066 & 0.206 \\ & & & & \\ 319.999 & 2.064 & 426.84 & 0.114 & 0.678 \\ 325.006 & 4.432 & 436.97 & 0.103 & 0.621 \\ 329.997 & 6.793 & 446.45 & 0.094 & 0.551 \\ 335.002 & 9.139 & 455.13 & 0.087 & 0.464 \\ 340.007 & 11.502 & 463.64 & 0.081 & 0.379 \\ 345.013 & 13.861 & 471.75 & 0.076 & 0.295 \\ 325.004 & 1.764 & 396.62 & 0.136 & 0.618 \\ 329.997 & 3.937 & 406.94 & 0.122 & 0.606 \\ 335.002 & 6.104 & 416.39 & 0.111 & 0.563 \\ 340.007 & 8.292 & 425.54 & 0.101 & 0.492 \\ 345.013 & 10.485 & 434.26 & 0.093 & 0.412 \\ 329.996 & 2.021 & 374.75 & 0.155 & 0.545 \\ 335.001 & 4.057 & 385.21 & 0.138 & 0.582 \\ 340.007 & 6.114 & 395.14 & 0.124 & 0.551 \\ 345.013 & 8.179 & 404.50 & 0.113 & 0.492 \\ 340.007 & 2.528 & 330.08 & 0.211 & 0.419 \\ 345.012 & 4.316 & 341.30 & 0.182 & 0.514 \\ 345.013 & 6.220 & 375.25 & 0.139 & 0.542 \\ & & & & \\ & & & & \\ 345.013 & 2.760 & 306.71 & 0.253 & 0.343\end{array}$


Table A.1-19. Measured speed of sound data for the system R-1234yf/1234ze(E) at a molar composition of $(0.33584 / 0.66416)$.

Listed are temperature $T$, pressure $P$, speed of sound $w$, relative combined, expanded $(k=2)$ state point uncertainty in the speed of sound $U_{c}$, and relative deviation from the mixture EOS. Average values for the replicate measurements at each $(T, P)$ state point are given.

$\begin{array}{crccc}T / \mathrm{K} & P / \mathrm{MPa} & w / \mathrm{m} \cdot \mathrm{s}^{-1} & U_{\mathrm{c}} / \% & \Delta_{w} / \% \\ & & & & \\ 230.014 & 0.267 & 806.10 & 0.039 & 0.224 \\ 240.003 & 0.542 & 761.96 & 0.041 & 0.170 \\ 245.003 & 0.534 & 739.27 & 0.043 & 0.147 \\ 250.004 & 0.543 & 716.62 & 0.044 & 0.099 \\ 229.999 & 0.263 & 806.07 & 0.039 & 0.214 \\ 235.008 & 0.521 & 784.77 & 0.040 & 0.216 \\ 254.995 & 0.542 & 694.03 & 0.046 & 0.049 \\ 259.987 & 0.551 & 671.60 & 0.048 & 0.000 \\ 264.995 & 0.547 & 649.01 & 0.051 & -0.053 \\ 269.992 & 2.854 & 645.90 & 0.050 & -0.054 \\ 269.991 & 2.931 & 646.50 & 0.050 & -0.057 \\ 274.995 & 6.403 & 652.94 & 0.049 & -0.073 \\ 279.999 & 9.895 & 659.50 & 0.047 & -0.098 \\ & & & & \\ 269.991 & 1.097 & 631.33 & 0.053 & -0.081 \\ 274.994 & 4.515 & 638.06 & 0.051 & -0.095 \\ 279.999 & 7.939 & 644.80 & 0.049 & -0.118 \\ 285.000 & 11.337 & 651.32 & 0.047 & -0.144 \\ 274.993 & 1.131 & 609.30 & 0.056 & -0.147 \\ 279.998 & 4.419 & 616.37 & 0.054 & -0.160 \\ 285.000 & 7.667 & 623.04 & 0.052 & -0.182 \\ 290.002 & 10.969 & 630.08 & 0.050 & -0.208 \\ 294.959 & & & \\ 295.000 & 4.323 & 594.65 & 0.057 & -0.232 \\ 290.002 & 7.458 & 601.75 & 0.054 & -0.250 \\ 294.998 & 10.584 & 608.69 & 0.052 & -0.268 \\ & & & & \\ 284.999 & 1.104 & 564.22 & 0.064 & -0.308 \\ & 4.108 & 571.77 & 0.061 & -0.312 \\ & 7.105 & 579.14 & 0.058 & -0.321 \\ & 10.097 & 586.32 & 0.055 & -0.331\end{array}$


Table A.1-19. (continued)

\begin{tabular}{|c|c|c|c|c|}
\hline$T / \mathrm{K}$ & $P / \mathrm{MPa}$ & $w / \mathrm{m} \cdot \mathrm{s}^{-1}$ & $U_{\mathrm{c}} / \%$ & $\Delta_{w} / \%$ \\
\hline 290.000 & 1.075 & 541.34 & 0.069 & -0.407 \\
\hline 294.997 & 3.934 & 549.11 & 0.065 & -0.398 \\
\hline 299.999 & 6.792 & 556.67 & 0.062 & -0.393 \\
\hline 304.997 & 9.651 & 564.09 & 0.059 & -0.392 \\
\hline 294.995 & 1.063 & 518.44 & 0.075 & -0.525 \\
\hline 299.998 & 3.801 & 526.62 & 0.071 & -0.489 \\
\hline 304.996 & 6.527 & 534.46 & 0.067 & -0.465 \\
\hline 309.999 & 9.236 & 541.90 & 0.063 & -0.446 \\
\hline 299.996 & 1.352 & 498.92 & 0.081 & -0.631 \\
\hline 304.996 & 3.965 & 507.27 & 0.076 & -0.570 \\
\hline 309.999 & 6.596 & 515.51 & 0.071 & -0.525 \\
\hline 314.994 & 9.206 & 523.32 & 0.067 & -0.488 \\
\hline 304.994 & 1.235 & 474.32 & 0.090 & -0.781 \\
\hline 309.998 & 3.713 & 483.04 & 0.084 & -0.674 \\
\hline 314.994 & 6.188 & 491.40 & 0.078 & -0.594 \\
\hline 320.003 & 8.666 & 499.42 & 0.074 & -0.536 \\
\hline 309.997 & 1.379 & 452.78 & 0.100 & -0.909 \\
\hline 314.993 & 3.723 & 461.69 & 0.092 & -0.755 \\
\hline 320.004 & 6.073 & 470.18 & 0.086 & -0.643 \\
\hline 325.009 & 8.419 & 478.31 & 0.080 & -0.564 \\
\hline 314.992 & 1.642 & 432.89 & 0.110 & -1.014 \\
\hline 320.002 & 3.877 & 442.08 & 0.101 & -0.813 \\
\hline 325.008 & 6.112 & 450.77 & 0.093 & -0.671 \\
\hline 329.999 & 8.337 & 459.02 & 0.087 & -0.573 \\
\hline 320.001 & 1.562 & 407.47 & 0.127 & -1.183 \\
\hline 325.007 & 3.651 & 416.91 & 0.115 & -0.905 \\
\hline 329.999 & 5.740 & 425.83 & 0.106 & -0.717 \\
\hline 335.003 & 7.817 & 434.09 & 0.098 & -0.590 \\
\hline 325.006 & 1.684 & 384.70 & 0.145 & -1.319 \\
\hline 329.998 & 3.647 & 394.49 & 0.131 & -0.968 \\
\hline 335.003 & 5.598 & 403.39 & 0.120 & -0.73 \\
\hline
\end{tabular}


Table A.1-19. (continued)

$\begin{array}{ccccc}T / \mathrm{K} & P / \mathrm{MPa} & w / \mathrm{m} \cdot \mathrm{s}^{-1} & U_{\mathrm{c}} / \% & \Delta_{w} / \% \\ 340.007 & 7.566 & 412.01 & 0.110 & -0.584 \\ 329.998 & 1.945 & 364.29 & 0.165 & -1.375 \\ 335.002 & 3.785 & 374.11 & 0.148 & -0.973 \\ 340.007 & 5.635 & 383.35 & 0.134 & -0.715 \\ 345.013 & 7.491 & 392.12 & 0.123 & -0.550 \\ & & & & \\ 335.001 & 2.049 & 340.38 & 0.197 & -1.462 \\ 340.006 & 3.780 & 350.84 & 0.174 & -0.979 \\ 345.012 & 5.516 & 360.52 & 0.155 & -0.682 \\ & & & & \\ 340.006 & 2.260 & 318.25 & 0.235 & -1.445 \\ 345.012 & 3.869 & 328.91 & 0.204 & -0.920 \\ 345.012 & 2.476 & 295.80 & 0.286 & -1.345\end{array}$


Table A.1-20. Measured speed of sound data for the system R-1234yf/1234ze(E) at a molar composition of $(0.66660 / 0.33340)$.

Listed are temperature $T$, pressure $P$, speed of sound $w$, relative combined, expanded $(k=2)$ state point uncertainty in the speed of sound $U_{c}$, and relative deviation from the mixture EOS. Average values for the replicate measurements at each $(T, P)$ state point are given. The different isochores are separated by blank lines.

$\begin{array}{crccc}T / \mathrm{K} & P / \mathrm{MPa} & w / \mathrm{m}^{-} \mathrm{s}^{-1} & U_{\mathrm{c}} / \% & \Delta_{w} / \% \\ & & & & \\ 229.998 & 0.450 & 777.99 & 0.041 & 0.128 \\ 235.008 & 0.591 & 756.13 & 0.042 & 0.161 \\ 240.004 & 0.607 & 733.50 & 0.043 & 0.156 \\ 245.003 & 0.616 & 710.97 & 0.045 & 0.143 \\ 250.005 & 0.624 & 688.51 & 0.047 & 0.119 \\ 254.994 & 0.792 & 667.55 & 0.049 & 0.100 \\ 259.987 & 4.524 & 674.41 & 0.047 & 0.041 \\ 269.993 & 11.961 & 687.78 & 0.045 & -0.081 \\ & & & & \\ 259.986 & 1.160 & 648.25 & 0.051 & 0.053 \\ 269.990 & 8.269 & 661.67 & 0.047 & -0.066 \\ 274.995 & 11.836 & 668.41 & 0.046 & -0.121 \\ 265.003 & 4.628 & 654.24 & 0.049 & -0.006 \\ & & & & \\ 264.994 & 1.127 & 625.75 & 0.054 & -0.002 \\ 269.991 & 4.572 & 633.05 & 0.052 & -0.061 \\ 274.995 & 8.070 & 640.62 & 0.049 & -0.119 \\ 280.000 & 11.531 & 647.83 & 0.048 & -0.167 \\ 2895 & & & \\ 269.991 & 1.128 & 603.60 & 0.057 & -0.066 \\ 274.994 & 4.428 & 611.11 & 0.054 & -0.119 \\ 280.000 & 7.740 & 618.59 & 0.052 & -0.168 \\ 285.001 & 11.049 & 625.95 & 0.050 & -0.211 \\ 274.993 & 1.123 & 581.33 & 0.061 & -0.139 \\ 279.999 & 4.282 & 589.10 & 0.058 & -0.181 \\ 285.000 & 7.446 & 596.77 & 0.055 & -0.220 \\ 290.002 & 10.608 & 604.28 & 0.053 & -0.253 \\ & & & & \\ 279.997 & 1.130 & 559.12 & 0.065 & -0.222 \\ & 4.140 & 567.06 & 0.061 & -0.249 \\ & 7.161 & 574.92 & 0.058 & -0.274 \\ & 10.166 & 582.50 & 0.056 & -0.294\end{array}$


Table A.1-20. (continued)

\begin{tabular}{|c|c|c|c|c|}
\hline$T / \mathrm{K}$ & $P / \mathrm{MPa}$ & $w / \mathrm{m} \cdot \mathrm{s}^{-1}$ & $U_{\mathrm{c}} / \%$ & $\Delta_{w} / \%$ \\
\hline 284.999 & 1.148 & 536.92 & 0.070 & -0.314 \\
\hline 290.001 & 4.005 & 545.01 & 0.066 & -0.315 \\
\hline 294.998 & 6.873 & 552.95 & 0.062 & -0.324 \\
\hline 299.999 & 9.747 & 560.75 & 0.059 & -0.330 \\
\hline 290.000 & 1.030 & 513.07 & 0.077 & -0.415 \\
\hline 294.997 & 3.758 & 521.57 & 0.072 & -0.393 \\
\hline 299.998 & 6.487 & 529.78 & 0.068 & -0.377 \\
\hline 304.998 & 9.212 & 537.70 & 0.064 & -0.366 \\
\hline 294.995 & 1.070 & 490.76 & 0.084 & -0.524 \\
\hline 299.998 & 3.690 & 499.76 & 0.078 & -0.475 \\
\hline 304.997 & 6.300 & 508.33 & 0.073 & -0.432 \\
\hline 309.998 & 8.904 & 516.52 & 0.069 & -0.401 \\
\hline 299.996 & 1.030 & 467.14 & 0.093 & -0.651 \\
\hline 304.996 & 3.494 & 476.27 & 0.086 & -0.555 \\
\hline 310.000 & 5.961 & 485.01 & 0.080 & -0.483 \\
\hline 314.993 & 8.401 & 493.16 & 0.075 & -0.424 \\
\hline 304.995 & 1.196 & 446.00 & 0.103 & -0.765 \\
\hline 309.998 & 3.530 & 455.33 & 0.095 & -0.624 \\
\hline 314.994 & 5.869 & 464.26 & 0.088 & -0.522 \\
\hline 320.004 & 8.211 & 472.78 & 0.082 & -0.446 \\
\hline 309.997 & 0.984 & 418.91 & 0.119 & -0.946 \\
\hline 314.993 & 3.165 & 428.60 & 0.108 & -0.738 \\
\hline 320.005 & 5.351 & 437.71 & 0.100 & -0.587 \\
\hline 325.008 & 7.532 & 446.32 & 0.092 & -0.482 \\
\hline 314.991 & 1.535 & 403.52 & 0.129 & -1.006 \\
\hline 320.002 & 3.623 & 413.31 & 0.116 & -0.768 \\
\hline 325.008 & 5.715 & 422.57 & 0.107 & -0.595 \\
\hline 329.999 & 7.793 & 431.19 & 0.098 & -0.473 \\
\hline 320.002 & 1.614 & 380.30 & 0.148 & -1.169 \\
\hline 325.008 & 3.572 & 390.39 & 0.133 & -0.859 \\
\hline 329.999 & 5.527 & 399.80 & 0.121 & -0.639 \\
\hline 335.004 & 7.472 & 408.44 & 0.111 & -0.487 \\
\hline
\end{tabular}




\section{A.1.4. Liquid-phase Thermal Conductivity}

The transient hot wire (THW) apparatus is an absolute technique used to measure the thermal conductivity. The apparatus described in this study ${ }^{82}$ can operate at temperatures ranging from 60 to $340 \mathrm{~K}$ and pressures up to $70 \mathrm{MPa}$. The basis of the THW apparatus are two platinum wires of differing lengths, which function as both heating elements and thermometers. The principle of the THW technique is to observe the temperature rise of the wire during a step power pulse through the wire over a short duration. The following equation,

$$
\Delta T_{i d}=\Delta T-\sum \delta T_{i}=\frac{q}{4 \pi \lambda} \ln \left(\frac{4 K}{a^{2} C} t\right)
$$

relates the measured temperature rise $\Delta T$ to the ideal temperature rise $\Delta T_{\text {id }}$ of an infinite line source where $q$ is the applied power, $\lambda$ is the thermal conductivity, $K$ is the thermal diffusivity, $a$ is the radius of the wire, $C$ is the exponential of Euler's constant, and $t$ is the time. The equation for the infinite line source is an ideal scenario assuming a wire of nearzero diameter and infinite length with zero heat capacity and the term $\Sigma \delta T_{\mathrm{i}}$ is the sum of corrections that are applied to the measured temperature rise. A more in-depth explanation of each correction required is described in the work of Healy et al. ${ }^{83}$

The apparatus consists of a measuring cell containing the hot wires situated in one arm of a Wheatstone bridge to measure the difference between the long-wire and short-wire resistances increases during heating; a beryllium copper pressure vessel rated to $70 \mathrm{MPa}$ and a cryostat to maintain the temperature complete the basic apparatus. Figure A.1-7 shows the arrangement of the long and short hot wires on the Wheatstone bridge. The two-wire arrangement used in this THW apparatus allows for the elimination of end effects. During measurements, an in-situ calibration is performed to correlate the wire resistances to their temperature. The heating of the platinum wires is done using a $1000 \mathrm{~Hz}$ alternating current power source to avoid polarization errors that may occur with ionic impurities in the refrigerants studied with bare hot wires.

Measurements are performed over a $1 \mathrm{~s}$ time period to minimize convective heat transfer and at five temperature rises to rule out any power level dependency. The data are measured isothermally from 200 to $340 \mathrm{~K}$ in $20 \mathrm{~K}$ increments. Increasing the system temperature causes the resistance of the long and short wires to increase. Therefore, prior to starting any measurements the Wheatstone bridge is balanced using decade resistors so that the voltage measured across the bridge reads zero. Saturated binary liquid refrigerant mixture samples of HFO-1234yf, HFC-134a. and HFO-1234ze(E) prepared in $300 \mathrm{~mL}$ transfer vessels were used to load the hot-wire system. The mixtures were prepared in such a manner that it minimized the vapor space in the transfer vessel. When loading the sample the transfer vessel was inverted to load from the liquid phase. Given the two-phase nature of the sample, flash calculations using REFPROP were performed to correct for modest changes in the bulk sample composition as the sample was withdrawn. The liquid phase composition changes were found to be between 0.0002 and 0.0005 mole fraction of the bulk sample composition. For each fluid measurements at nine pressures were performed along an isotherm starting from roughly $0.5 \mathrm{MPa}$ above the bubble point pressure to $50 \mathrm{MPa}$. For mixtures containing HFO-1234yf the pressure was limited to $12 \mathrm{MPa}$ because this component has the potential to 
polymerize at high pressures. The combined expanded uncertainty of the thermal conductivity measurement for the liquid mixtures measured here is less than $1 \%$. Figure A.18 shows the relationship between the thermal conductivity and density for each binary mixture.

Representative measured data are presented in Tables A.1-21 through A.1-26. For the sake of brevity, the tables present a single measured point at each nominal pressure, rather than the five distinct temperature rises actually measured. All of the measured data will be presented in a forthcoming journal paper.

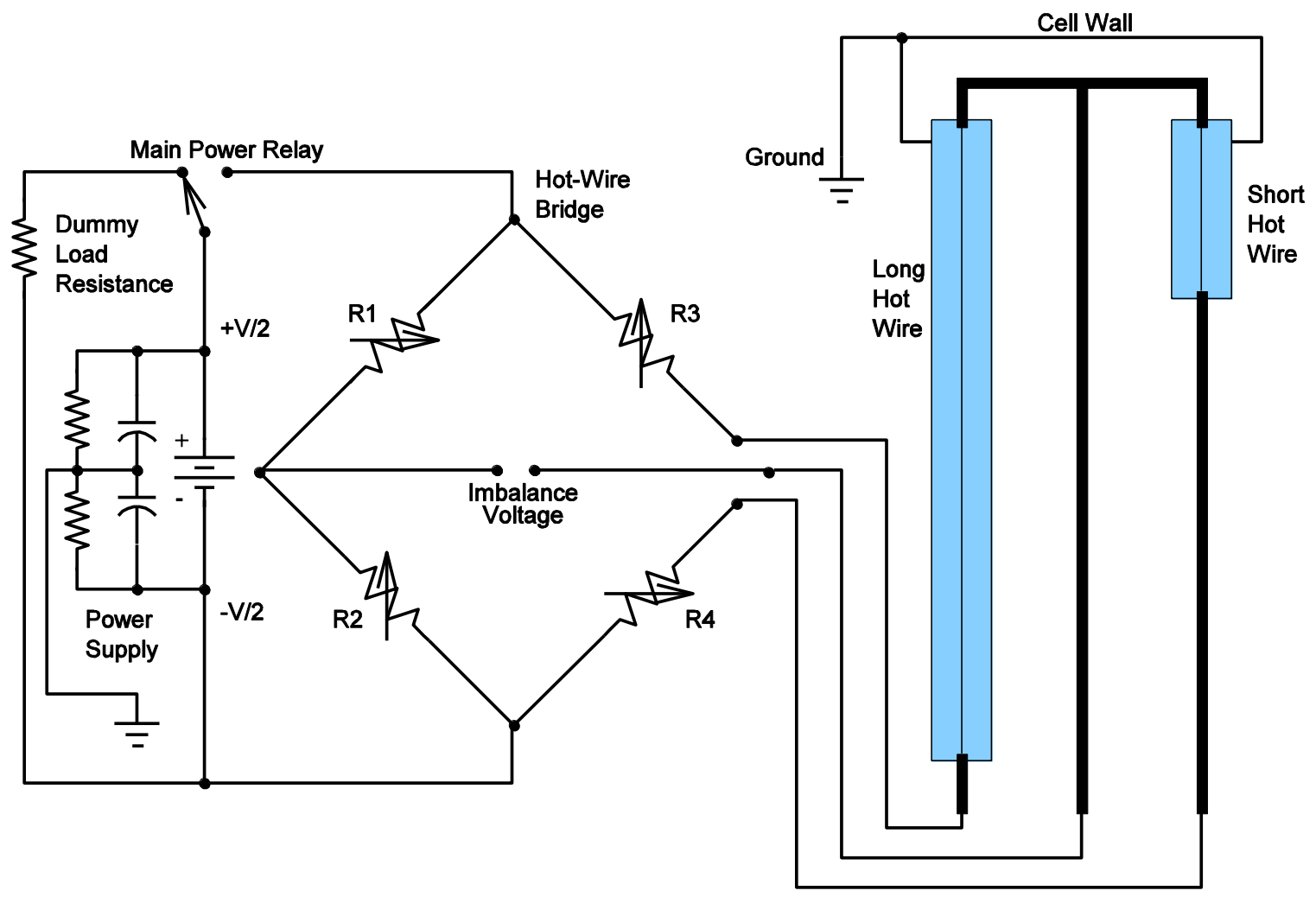

Figure A.1-7. Circuit diagram of the Wheatstone bridge and long and short hot wire arrangement. 

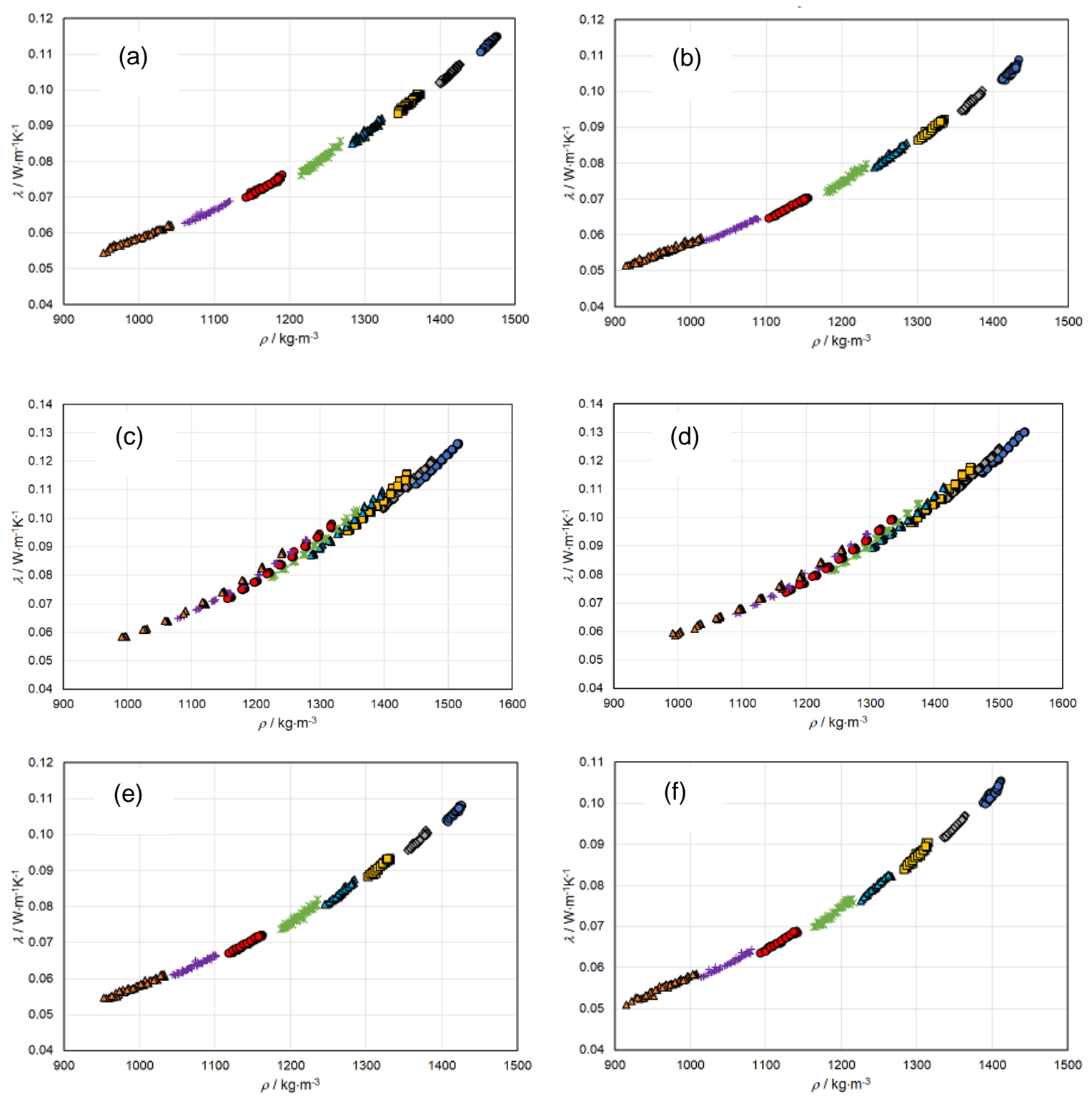

○ $200 \mathrm{~K} \diamond 220 \mathrm{~K}$ 口 $240 \mathrm{~K} \Delta 260 \mathrm{~K} * 280 \mathrm{~K}$ ○ $300 \mathrm{~K}+320 \mathrm{~K} \Delta 340 \mathrm{~K}$

Figure A.1-8. Effect of density on the thermal conductivity for binary mixtures of R-1234yf, $\mathrm{R}-134 \mathrm{a}$, and R-1234ze(E) at temperatures ranging from $200 \mathrm{~K}$ to $340 \mathrm{~K}$.

The mixtures are: (a) R-1234yf/134a (0.320/0.680 molar); (b) R-1234yf/134a (0.647/0.353 molar); (c) R-134a/1234ze(E) (0.334/0.666 molar); (d) R-134a/1234ze(E) (0.663/0.337 molar); (e) R-1234yf/1234ze(E) (0.323/0.677 molar); (f) R-1234yf/1234ze(E) (0.642/0.358 molar). 
Table A.1-21. Representative thermal conductivity data measured for the system $\mathrm{R}-1234 \mathrm{yf} / 134 \mathrm{a}$ at a molar composition of $(0.320 / 0.680)$.

\begin{tabular}{|c|c|c|c|c|c|}
\hline$T / \mathrm{K}$ & $P / \mathrm{MPa}$ & $\lambda / \mathrm{W} \cdot \mathrm{m}^{-1} \mathrm{~K}^{-1}$ & $T / \mathrm{K}$ & $P / \mathrm{MPa}$ & $\lambda / \mathrm{W} \cdot \mathrm{m}^{-1} \mathrm{~K}^{-1}$ \\
\hline 201.920 & 2.155 & 0.11140 & 282.240 & 2.300 & 0.07806 \\
\hline 201.919 & 2.152 & 0.11161 & 282.224 & 1.078 & 0.07749 \\
\hline 201.893 & 3.573 & 0.11198 & 282.175 & 2.126 & 0.07834 \\
\hline 201.891 & 3.573 & 0.11179 & 282.356 & 1.050 & 0.07758 \\
\hline 201.557 & 8.921 & 0.11391 & 282.361 & 2.070 & 0.07789 \\
\hline 202.247 & 11.885 & 0.11494 & 282.260 & 3.289 & 0.07888 \\
\hline 202.271 & 10.415 & 0.11427 & 282.264 & 4.704 & 0.07995 \\
\hline 202.347 & 7.678 & 0.11310 & 282.182 & 6.006 & 0.08076 \\
\hline 202.344 & 5.055 & 0.11286 & 282.197 & 7.420 & 0.08166 \\
\hline 202.353 & 0.950 & 0.11111 & 282.118 & 8.924 & 0.08273 \\
\hline 222.435 & 12.026 & 0.10695 & 282.039 & 11.634 & 0.08412 \\
\hline 222.516 & 10.314 & 0.10628 & 302.260 & 1.157 & 0.07035 \\
\hline 222.472 & 9.034 & 0.10560 & 302.271 & 2.011 & 0.07119 \\
\hline 222.504 & 7.633 & 0.10502 & 302.270 & 2.888 & 0.07171 \\
\hline 222.514 & 6.297 & 0.10455 & 302.273 & 3.599 & 0.07206 \\
\hline 222.560 & 4.869 & 0.10364 & 302.264 & 4.346 & 0.07273 \\
\hline 222.573 & 3.656 & 0.10321 & 302.241 & 5.327 & 0.07347 \\
\hline 222.559 & 2.334 & 0.10277 & 302.230 & 6.113 & 0.07382 \\
\hline 222.593 & 1.040 & 0.10199 & 302.218 & 7.070 & 0.07485 \\
\hline 242.683 & 11.940 & 0.09869 & 302.198 & 7.905 & 0.07525 \\
\hline 242.739 & 10.479 & 0.09787 & 322.071 & 1.909 & 0.06311 \\
\hline 242.757 & 8.972 & 0.09692 & 321.974 & 2.440 & 0.06397 \\
\hline 242.764 & 6.293 & 0.09659 & 322.022 & 3.119 & 0.06467 \\
\hline 242.741 & 7.673 & 0.09629 & 322.015 & 3.843 & 0.06512 \\
\hline 242.782 & 5.024 & 0.09540 & 321.927 & 4.558 & 0.06579 \\
\hline 242.799 & 3.483 & 0.09499 & 321.915 & 5.342 & 0.06625 \\
\hline 242.801 & 2.154 & 0.09437 & 321.921 & 6.093 & 0.06671 \\
\hline 242.826 & 0.969 & 0.09325 & 321.921 & 7.042 & 0.06820 \\
\hline 262.143 & 11.959 & 0.09162 & 321.900 & 7.920 & 0.06890 \\
\hline 262.172 & 10.621 & 0.09055 & 342.135 & 2.579 & 0.05605 \\
\hline 262.188 & 8.969 & 0.08970 & 342.208 & 2.979 & 0.05646 \\
\hline 262.159 & 7.513 & 0.08927 & 342.181 & 3.464 & 0.05722 \\
\hline 262.192 & 6.185 & 0.08881 & 342.175 & 4.056 & 0.05812 \\
\hline 262.226 & 4.808 & 0.08875 & 342.031 & 4.670 & 0.05902 \\
\hline 262.208 & 4.829 & 0.08812 & 342.399 & 5.405 & 0.05956 \\
\hline 262.233 & 3.524 & 0.08651 & 342.420 & 6.237 & 0.06018 \\
\hline 262.240 & 2.081 & 0.08620 & 342.382 & 6.964 & 0.06089 \\
\hline 262.218 & 0.990 & 0.08561 & 342.340 & 7.878 & 0.06236 \\
\hline
\end{tabular}


Table A.1-22. Representative thermal conductivity data measured for the system R$1234 \mathrm{yf} / 134 \mathrm{a}$ at a molar composition of $(0.647 / 0.353)$.

\begin{tabular}{|c|c|c|c|c|c|}
\hline$T / \mathrm{K}$ & $P / \mathrm{MPa}$ & $\lambda / \mathrm{W} \cdot \mathrm{m}^{-1} \mathrm{~K}^{-1}$ & $T / \mathrm{K}$ & $P / \mathrm{MPa}$ & $\lambda / \mathrm{W} \cdot \mathrm{m}^{-1} \mathrm{~K}^{-1}$ \\
\hline 202.029 & 1.154 & 0.10308 & 281.872 & 3.306 & 0.07380 \\
\hline 202.036 & 2.153 & 0.10403 & 281.818 & 4.506 & 0.07453 \\
\hline 201.465 & 3.573 & 0.10469 & 281.905 & 6.008 & 0.07547 \\
\hline 201.725 & 3.572 & 0.10469 & 281.875 & 7.681 & 0.07612 \\
\hline 201.472 & 4.962 & 0.10517 & 281.809 & 7.700 & 0.07615 \\
\hline 202.101 & 4.975 & 0.10469 & 281.128 & 8.935 & 0.07827 \\
\hline 202.049 & 6.238 & 0.10480 & 282.007 & 10.353 & 0.07781 \\
\hline 202.034 & 7.559 & 0.10495 & 282.028 & 11.999 & 0.07857 \\
\hline 202.039 & 9.049 & 0.10585 & 302.464 & 1.299 & 0.06506 \\
\hline 202.015 & 10.412 & 0.10713 & 302.105 & 1.997 & 0.06605 \\
\hline 202.060 & 11.793 & 0.10701 & 302.248 & 2.737 & 0.06654 \\
\hline 222.284 & 1.181 & 0.09488 & 302.133 & 3.483 & 0.06698 \\
\hline 222.303 & 2.158 & 0.09514 & 301.907 & 4.298 & 0.06796 \\
\hline 222.305 & 3.461 & 0.09616 & 301.874 & 5.131 & 0.06844 \\
\hline 222.292 & 4.773 & 0.09669 & 302.199 & 6.288 & 0.06891 \\
\hline 222.272 & 6.329 & 0.09752 & 302.105 & 7.096 & 0.06981 \\
\hline 222.275 & 7.767 & 0.09758 & 302.176 & 8.035 & 0.07007 \\
\hline 222.262 & 9.136 & 0.09832 & 322.289 & 1.638 & 0.05874 \\
\hline 222.251 & 10.532 & 0.09885 & 322.225 & 2.316 & 0.05941 \\
\hline 222.244 & 11.818 & 0.09926 & 322.172 & 2.976 & 0.06032 \\
\hline 242.508 & 1.033 & 0.08689 & 322.219 & 3.707 & 0.06100 \\
\hline 242.486 & 2.304 & 0.08751 & 322.121 & 4.434 & 0.06180 \\
\hline 242.532 & 3.531 & 0.08780 & 322.001 & 5.249 & 0.06241 \\
\hline 242.530 & 4.935 & 0.08832 & 322.139 & 6.155 & 0.06322 \\
\hline 242.540 & 6.142 & 0.08895 & 322.083 & 7.072 & 0.06397 \\
\hline 242.523 & 7.541 & 0.08974 & 322.045 & 8.031 & 0.06449 \\
\hline 242.500 & 8.973 & 0.09024 & 342.621 & 2.401 & 0.05164 \\
\hline 242.495 & 10.579 & 0.09077 & 342.534 & 2.847 & 0.05208 \\
\hline 242.472 & 11.946 & 0.09184 & 342.736 & 3.460 & 0.05328 \\
\hline 262.259 & 1.077 & 0.07923 & 342.613 & 3.980 & 0.05413 \\
\hline 262.294 & 2.162 & 0.08018 & 342.476 & 3.966 & 0.05440 \\
\hline 262.254 & 3.386 & 0.08084 & 342.501 & 4.729 & 0.05572 \\
\hline 262.200 & 4.727 & 0.08119 & 342.640 & 4.770 & 0.05548 \\
\hline 262.261 & 6.172 & 0.08160 & 342.474 & 5.410 & 0.05584 \\
\hline 262.225 & 7.558 & 0.08285 & 342.357 & 6.220 & 0.05716 \\
\hline 262.176 & 9.057 & 0.08327 & 342.474 & 7.052 & 0.05765 \\
\hline 262.127 & 10.486 & 0.08422 & 341.938 & 7.035 & 0.05796 \\
\hline 262.088 & 11.886 & 0.08514 & 342.045 & 7.968 & 0.05841 \\
\hline 281.922 & 1.103 & 0.07236 & 341.993 & 7.955 & 0.05900 \\
\hline 281.860 & 2.032 & 0.07294 & 342.226 & 2.698 & 0.05245 \\
\hline
\end{tabular}


Table A.1-23. Representative thermal conductivity data measured for the system R-134a/1234ze(E)) at a molar composition of (0.334/0.666).

\begin{tabular}{|c|c|c|c|c|c|}
\hline$T / \mathrm{K}$ & $P / \mathrm{MPa}$ & $\lambda / \mathrm{W} \cdot \mathrm{m}^{-1} \mathrm{~K}^{-1}$ & $T / \mathrm{K}$ & $P / \mathrm{MPa}$ & $\lambda / \mathrm{W} \cdot \mathrm{m}^{-1} \mathrm{~K}^{-1}$ \\
\hline 202.306 & 1.006 & 0.11192 & 282.586 & 1.027 & 0.07981 \\
\hline 202.356 & 6.092 & 0.11376 & 282.497 & 5.018 & 0.08243 \\
\hline 202.339 & 11.496 & 0.11505 & 282.413 & 9.586 & 0.08472 \\
\hline 202.328 & 17.141 & 0.11665 & 282.314 & 14.660 & 0.08730 \\
\hline 202.325 & 23.189 & 0.11893 & 282.249 & 20.585 & 0.09057 \\
\hline 202.319 & 29.514 & 0.12054 & 282.287 & 27.011 & 0.09311 \\
\hline 202.317 & 36.292 & 0.12279 & 282.114 & 34.280 & 0.09661 \\
\hline 202.210 & 42.843 & 0.12437 & 282.010 & 41.528 & 0.09871 \\
\hline 202.207 & 50.101 & 0.12622 & 282.115 & 50.057 & 0.10198 \\
\hline 222.169 & 1.056 & 0.10367 & 302.384 & 0.961 & 0.07264 \\
\hline 222.565 & 5.931 & 0.10541 & 302.252 & 4.796 & 0.07501 \\
\hline 222.552 & 11.136 & 0.10792 & 302.093 & 8.766 & 0.07760 \\
\hline 222.536 & 16.705 & 0.10965 & 302.017 & 13.756 & 0.08079 \\
\hline 222.523 & 22.545 & 0.11217 & 302.041 & 19.225 & 0.08340 \\
\hline 222.502 & 29.147 & 0.11436 & 301.872 & 25.374 & 0.08657 \\
\hline 222.490 & 35.966 & 0.11594 & 301.831 & 32.621 & 0.09001 \\
\hline 222.477 & 42.648 & 0.11734 & 301.760 & 40.836 & 0.09354 \\
\hline 222.474 & 50.056 & 0.12027 & 301.735 & 50.102 & 0.09730 \\
\hline 242.282 & 1.066 & 0.09586 & 322.937 & 1.401 & 0.06497 \\
\hline 242.262 & 5.686 & 0.09794 & 322.899 & 4.685 & 0.06805 \\
\hline 242.240 & 10.677 & 0.09960 & 322.696 & 8.283 & 0.07091 \\
\hline 242.230 & 16.211 & 0.10246 & 322.692 & 12.567 & 0.07383 \\
\hline 242.205 & 22.160 & 0.10487 & 322.674 & 17.834 & 0.07684 \\
\hline 242.183 & 28.493 & 0.10626 & 322.579 & 23.979 & 0.08026 \\
\hline 242.162 & 35.235 & 0.10873 & 322.458 & 31.757 & 0.08405 \\
\hline 242.135 & 42.241 & 0.11054 & 321.971 & 40.469 & 0.08811 \\
\hline 242.117 & 50.302 & 0.11354 & 321.578 & 49.979 & 0.09222 \\
\hline 262.459 & 1.107 & 0.08759 & 342.146 & 2.213 & 0.05862 \\
\hline 262.412 & 5.364 & 0.08999 & 341.996 & 4.487 & 0.06087 \\
\hline 262.317 & 10.428 & 0.09243 & 341.895 & 7.685 & 0.06391 \\
\hline 262.270 & 15.664 & 0.09503 & 342.268 & 7.683 & 0.06405 \\
\hline 262.217 & 21.652 & 0.09751 & 341.838 & 11.489 & 0.06754 \\
\hline 262.170 & 27.658 & 0.09965 & 341.916 & 16.350 & 0.07034 \\
\hline 262.124 & 34.362 & 0.10199 & 341.899 & 22.524 & 0.07403 \\
\hline 262.079 & 42.081 & 0.10489 & 342.360 & 22.522 & 0.07416 \\
\hline \multirow[t]{3}{*}{262.023} & 50.155 & 0.10792 & 341.766 & 30.150 & 0.07793 \\
\hline & & & 341.625 & 39.113 & 0.08293 \\
\hline & & & 341.507 & 50.178 & 0.08820 \\
\hline
\end{tabular}


Table A.1-24. Representative thermal conductivity data measured for the system R-134a/1234ze(E)) at a molar composition of (0.663/0.337).

\begin{tabular}{|c|c|c|c|c|c|}
\hline$T / \mathrm{K}$ & $P / \mathrm{MPa}$ & $\lambda / \mathrm{W} \cdot \mathrm{m}^{-1} \mathrm{~K}^{-1}$ & $T / \mathrm{K}$ & $P / \mathrm{MPa}$ & $\lambda / \mathrm{W} \cdot \mathrm{m}^{-1} \mathrm{~K}^{-1}$ \\
\hline 202.414 & 0.987 & 0.11549 & 282.675 & 0.976 & 0.08186 \\
\hline 202.412 & 6.060 & 0.11748 & 282.606 & 5.044 & 0.08448 \\
\hline 202.410 & 11.549 & 0.11964 & 282.552 & 9.791 & 0.08700 \\
\hline 202.411 & 17.236 & 0.12010 & 282.475 & 14.660 & 0.08946 \\
\hline 202.418 & 23.225 & 0.12327 & 282.413 & 20.690 & 0.09308 \\
\hline 202.342 & 29.496 & 0.12454 & 282.382 & 26.559 & 0.09525 \\
\hline 202.352 & 35.981 & 0.12704 & 282.328 & 33.614 & 0.09844 \\
\hline 202.367 & 42.732 & 0.12895 & 282.272 & 41.127 & 0.10155 \\
\hline 202.367 & 49.945 & 0.12998 & 282.249 & 49.838 & 0.10511 \\
\hline 222.064 & 1.065 & 0.10711 & 302.774 & 1.302 & 0.07414 \\
\hline 222.214 & 5.868 & 0.10911 & 302.671 & 4.832 & 0.07664 \\
\hline 222.257 & 11.181 & 0.11148 & 302.597 & 8.887 & 0.07975 \\
\hline 222.241 & 16.618 & 0.11292 & 302.619 & 13.716 & 0.08232 \\
\hline 222.216 & 22.787 & 0.11515 & 302.479 & 19.229 & 0.08542 \\
\hline 222.202 & 28.987 & 0.11771 & 302.335 & 25.600 & 0.08900 \\
\hline 222.192 & 35.725 & 0.11943 & 302.237 & 32.919 & 0.09191 \\
\hline 222.017 & 42.464 & 0.12160 & 302.301 & 40.862 & 0.09563 \\
\hline 221.978 & 50.005 & 0.12412 & 302.176 & 49.796 & 0.09927 \\
\hline 242.597 & 1.100 & 0.09880 & 322.776 & 1.570 & 0.06657 \\
\hline 242.565 & 5.653 & 0.10123 & 322.781 & 4.653 & 0.06924 \\
\hline 242.536 & 10.781 & 0.10295 & 322.086 & 8.416 & 0.07251 \\
\hline 242.521 & 16.107 & 0.10506 & 321.956 & 13.014 & 0.07611 \\
\hline 242.498 & 22.116 & 0.10631 & 321.887 & 17.630 & 0.07869 \\
\hline 242.473 & 28.225 & 0.10981 & 322.338 & 24.025 & 0.08251 \\
\hline 242.456 & 34.938 & 0.11133 & 321.852 & 39.832 & 0.09054 \\
\hline 242.437 & 42.219 & 0.11428 & 322.164 & 50.069 & 0.09430 \\
\hline 242.419 & 50.130 & 0.11722 & 342.332 & 2.379 & 0.05897 \\
\hline 262.970 & 1.072 & 0.08986 & 342.189 & 4.494 & 0.06231 \\
\hline 262.931 & 5.384 & 0.09218 & 342.127 & 7.289 & 0.06531 \\
\hline 262.887 & 10.105 & 0.09439 & 342.046 & 11.277 & 0.06809 \\
\hline 262.853 & 15.441 & 0.09723 & 341.971 & 16.146 & 0.07184 \\
\hline 262.800 & 21.204 & 0.09959 & 341.882 & 22.092 & 0.07578 \\
\hline 262.758 & 27.697 & 0.10202 & 341.772 & 29.594 & 0.07943 \\
\hline 262.703 & 34.550 & 0.10423 & 341.695 & 38.837 & 0.08415 \\
\hline 262.666 & 41.737 & 0.10805 & 341.629 & 50.136 & 0.08911 \\
\hline 262.612 & 49.833 & 0.11047 & & & \\
\hline
\end{tabular}


Table A.1-25. Representative thermal conductivity data measured for the system R-1234yf/1234ze(E) at a molar composition of (0.323/0.677).

\begin{tabular}{|c|c|c|c|c|c|}
\hline$T / \mathrm{K}$ & $P / \mathrm{MPa}$ & $\lambda / \mathrm{W} \cdot \mathrm{m}^{-1} \mathrm{~K}^{-1}$ & $T / \mathrm{K}$ & $P / \mathrm{MPa}$ & $\lambda / \mathrm{W} \cdot \mathrm{m}^{-1} \mathrm{~K}^{-1}$ \\
\hline 202.374 & 1.088 & 0.10437 & 282.065 & 1.039 & 0.07407 \\
\hline 202.342 & 2.401 & 0.10476 & 282.045 & 2.153 & 0.07456 \\
\hline 202.348 & 3.644 & 0.10480 & 282.055 & 3.428 & 0.07570 \\
\hline 202.400 & 4.960 & 0.10560 & 281.981 & 4.771 & 0.07657 \\
\hline 202.395 & 6.309 & 0.10606 & 281.991 & 6.062 & 0.07716 \\
\hline 202.587 & 8.086 & 0.10660 & 281.947 & 7.336 & 0.07787 \\
\hline 202.579 & 9.299 & 0.10688 & 282.026 & 8.962 & 0.07881 \\
\hline 202.570 & 10.592 & 0.10694 & 281.959 & 10.640 & 0.07939 \\
\hline 202.566 & 11.912 & 0.10731 & 282.001 & 12.325 & 0.08024 \\
\hline 222.322 & 1.028 & 0.09612 & 302.454 & 1.118 & 0.06715 \\
\hline 222.311 & 2.300 & 0.09663 & 302.396 & 1.865 & 0.06788 \\
\hline 222.299 & 3.552 & 0.09710 & 302.422 & 2.697 & 0.06870 \\
\hline 222.277 & 4.896 & 0.09752 & 302.393 & 3.469 & 0.06919 \\
\hline 222.271 & 6.165 & 0.09839 & 302.370 & 4.307 & 0.06956 \\
\hline 222.256 & 7.719 & 0.09877 & 302.562 & 5.164 & 0.07011 \\
\hline 222.234 & 9.091 & 0.09904 & 302.512 & 6.104 & 0.07065 \\
\hline 222.226 & 10.750 & 0.10019 & 302.479 & 7.129 & 0.07158 \\
\hline 222.057 & 12.065 & 0.09975 & 302.441 & 8.075 & 0.07193 \\
\hline 242.560 & 1.127 & 0.08892 & 322.230 & 1.463 & 0.06079 \\
\hline 242.557 & 2.313 & 0.08932 & 322.256 & 2.145 & 0.06158 \\
\hline 242.543 & 3.553 & 0.08959 & 322.219 & 2.828 & 0.06223 \\
\hline 242.512 & 4.916 & 0.09007 & 322.138 & 3.480 & 0.06300 \\
\hline 242.490 & 6.300 & 0.09110 & 322.182 & 4.418 & 0.06493 \\
\hline 242.465 & 7.672 & 0.09171 & 322.150 & 5.293 & 0.06421 \\
\hline 242.436 & 8.979 & 0.09241 & 322.123 & 6.224 & 0.06504 \\
\hline 242.498 & 10.373 & 0.09297 & 322.113 & 7.045 & 0.06538 \\
\hline 242.482 & 11.940 & 0.09308 & 322.105 & 8.047 & 0.06607 \\
\hline 262.821 & 1.020 & 0.08055 & 342.054 & 2.207 & 0.05453 \\
\hline 262.331 & 2.316 & 0.08159 & 341.934 & 2.733 & 0.05601 \\
\hline 262.401 & 3.564 & 0.08212 & 341.986 & 3.349 & 0.05687 \\
\hline 262.302 & 4.849 & 0.08288 & 341.960 & 4.033 & 0.05685 \\
\hline 262.301 & 6.199 & 0.08343 & 341.881 & 4.655 & 0.05804 \\
\hline 262.222 & 7.587 & 0.08538 & 341.812 & 5.465 & 0.05821 \\
\hline 262.133 & 8.944 & 0.08527 & 342.373 & 6.254 & 0.05875 \\
\hline 262.098 & 10.612 & 0.08587 & 341.873 & 7.094 & 0.06015 \\
\hline 262.180 & 11.997 & 0.08652 & 341.901 & 8.002 & 0.06093 \\
\hline
\end{tabular}


Table A.1-26. Representative thermal conductivity data measured for the system $\mathrm{R}-1234 \mathrm{yf} / 1234 \mathrm{ze}(\mathrm{E})$ at a molar composition of $(0.642 / 0.358)$.

\begin{tabular}{|c|c|c|c|c|c|}
\hline$T / \mathrm{K}$ & $P / \mathrm{MPa}$ & $\lambda / \mathrm{W} \cdot \mathrm{m}^{-1} \mathrm{~K}^{-1}$ & $T / \mathrm{K}$ & $P / \mathrm{MPa}$ & $\lambda / \mathrm{W} \cdot \mathrm{m}^{-1} \mathrm{~K}^{-1}$ \\
\hline 202.210 & 1.019 & 0.10063 & 282.395 & 0.916 & 0.07010 \\
\hline 202.133 & 2.678 & 0.10049 & 282.367 & 2.179 & 0.07087 \\
\hline 202.191 & 3.759 & 0.10202 & 282.313 & 3.392 & 0.07173 \\
\hline 202.197 & 5.086 & 0.10246 & 282.261 & 4.656 & 0.07257 \\
\hline 202.226 & 6.239 & 0.10248 & 282.139 & 6.225 & 0.07349 \\
\hline 202.196 & 7.850 & 0.10265 & 282.141 & 7.739 & 0.07467 \\
\hline 202.180 & 9.424 & 0.10287 & 282.038 & 9.183 & 0.07596 \\
\hline 201.797 & 10.729 & 0.10362 & 282.083 & 10.489 & 0.07641 \\
\hline 201.801 & 12.035 & 0.10472 & 282.106 & 11.877 & 0.07667 \\
\hline 222.731 & 1.046 & 0.09173 & 302.587 & 1.090 & 0.06385 \\
\hline 222.719 & 2.361 & 0.09268 & 302.518 & 1.939 & 0.06494 \\
\hline 222.702 & 3.690 & 0.09303 & 302.442 & 2.694 & 0.06512 \\
\hline 222.688 & 4.991 & 0.09363 & 302.357 & 3.521 & 0.06579 \\
\hline 222.672 & 6.293 & 0.09420 & 302.380 & 4.214 & 0.06629 \\
\hline 222.659 & 7.651 & 0.09471 & 302.283 & 5.050 & 0.06706 \\
\hline 222.506 & 9.142 & 0.09493 & 302.314 & 6.072 & 0.06753 \\
\hline 222.479 & 10.635 & 0.09549 & 302.290 & 7.078 & 0.06838 \\
\hline 222.458 & 12.078 & 0.09613 & 302.250 & 7.996 & 0.06877 \\
\hline 242.358 & 1.133 & 0.08430 & 322.788 & 1.475 & 0.05775 \\
\hline 242.363 & 2.377 & 0.08508 & 322.756 & 2.215 & 0.05866 \\
\hline 242.389 & 3.632 & 0.08568 & 322.753 & 2.789 & 0.05912 \\
\hline 242.259 & 4.942 & 0.08661 & 322.657 & 3.256 & 0.05958 \\
\hline 242.286 & 6.294 & 0.08717 & 322.645 & 4.395 & 0.06051 \\
\hline 242.307 & 7.642 & 0.08788 & 322.641 & 5.290 & 0.06141 \\
\hline 242.242 & 8.910 & 0.08816 & 322.514 & 6.137 & 0.06193 \\
\hline 242.208 & 10.456 & 0.08835 & 322.532 & 7.099 & 0.06279 \\
\hline 242.189 & 12.002 & 0.08916 & 322.436 & 7.960 & 0.06353 \\
\hline 262.665 & 6.173 & 0.07972 & 343.282 & 2.172 & 0.05105 \\
\hline 262.630 & 1.147 & 0.07707 & 343.167 & 2.801 & 0.05272 \\
\hline 262.645 & 2.253 & 0.07758 & 343.087 & 3.351 & 0.05326 \\
\hline 262.596 & 3.544 & 0.07877 & 342.997 & 4.040 & 0.05464 \\
\hline 262.543 & 4.833 & 0.07958 & 343.920 & 4.956 & 0.05526 \\
\hline 262.556 & 7.515 & 0.08049 & 343.259 & 5.456 & 0.05596 \\
\hline 262.513 & 9.047 & 0.08141 & 342.640 & 6.125 & 0.05670 \\
\hline 262.471 & 10.549 & 0.08238 & 342.593 & 7.006 & 0.05736 \\
\hline 262.415 & 11.983 & 0.08226 & 342.450 & 7.928 & 0.05830 \\
\hline
\end{tabular}




\section{A.1.5. Mixture Preparation}

All of the property measurements rely on preparation of the sample mixtures and accurate determination of their composition. The $(P, \rho, T, x)$ and speed-of-sound measurements utilized gas-phase mixtures, while the VLE and thermal conductivity measurements utilized liquid-phase mixtures. The mixtures were prepared gravimetrically (by weighing) to achieve low uncertainties in the composition.

The pure-fluid refrigerants used to prepare the mixtures were used as received except that we degassed them (prior to preparing the mixtures) by freezing the pure components in liquid nitrogen, evacuating the vapor space, and thawing; this sequence was repeated until the residual pressure over the frozen sample was less than $0.01 \mathrm{~Pa}$. We analyzed the pure-fluid refrigerants in-house using gas chromatography with mass spectrometry (GC-MS) and found no significant impurities.

The gas-phase blends were prepared in aluminum gas cylinders of approximately 6,10 or 13 $\mathrm{L}$ internal volume depending on the pressure of the blend. The sample mass was determined by a double substitution weighing design as described by Harris andTorres ${ }^{84}$ with a nearly identical "tare" or reference cylinder serving as the main substitution mass. Further details are provided by Richter and McLinden. ${ }^{85}$ The uncertainty of the measured gas-phase mixture compositions, arising from the weighings, was 0.0001 mole fraction. The sample cylinders were loaded to pressures corresponding to the dew-point pressure at $T=293.15 \mathrm{~K}$. There may have been a small amount of liquid in the sample cylinders after filling, but they were heated continuously to $T>313 \mathrm{~K}$ for the duration of the testing to ensure that only singlephase vapor was present. Due to sorption effects, the composition of the sample in the measuring cell could be different from that calculated from the sample masses loaded into the sample cylinder, and this contributed an additional uncertainty of 0.0002 mole fraction. The combined, expanded uncertainty was estimated to be 0.00022 mole fraction.

The liquid-phase mixtures were prepared in $300 \mathrm{~mL}$ stainless steel cylinders. The fluid with the higher boiling point was added first, then the second component. The vapor space above the mixture samples was degassed by freezing the sample with liquid nitrogen and opening the cylinder to vacuum. After evacuation, the sample was then heated to drive volatile impurities (such as air) into the vapor space. The entire cycle (freezing, evacuation, and heating) was repeated a minimum of three times for each sample. Mixtures were prepared with the goal of filling the sample cylinder with about $280 \mathrm{~mL}$ of liquid at ambient temperature. Thus, in each completed mixture cylinder there was a vapor space above the liquid phase.

A balance with a precision of $0.1 \mathrm{mg}$ was used in the preparation of the liquid-phase mixtures. Utilizing the double-substitution weighing design of Harris and Torres ${ }^{84}$ measurement of the mass of each component consisted of weighing four masses: (1) a reference cylinder of approximately the same mass and volume as the empty sample cylinder, (2) the sample cylinder, (3) the sample cylinder plus a 20 g sensitivity weight, and (4) the reference cylinder plus the $20 \mathrm{~g}$ sensitivity weight. This weighing sequence was repeated four times for each mass determination. The density of ambient air was calculated based on measurements of temperature, pressure, and relative humidity, and the weighings were 
corrected for the effects of air buoyancy. The standard deviation of the repeat weighings was at most $1.5 \mathrm{mg}$. The uncertainty in the composition due to the weighings was less than 0.0001 mole fraction. Because of the two-phase nature of the liquid-phase samples and because the weighings could determine only the overall (bulk) composition of the sample it was necessary to estimate the change in composition caused by the liquid/vapor fractionation inside the cylinder. This change was less than 0.0005 mole fraction, and its standard uncertainty was estimated to be less than 0.0001 mole fraction. Thus, the combined, expanded uncertainty in composition of the liquid-phase samples was estimated to be less than 0.00028 mole fraction.

\section{A.1-6. Mixture Modeling}

The multi-fluid modeling used in NIST REFPROP yields the most accurate mixture models available today. This approach combines the most accurate pure fluid equations of state with reducing and departure functions to correct for the changes to thermodynamics caused by mixture interactions. This model forms the basis of the GERG-2008 model, used for custody transfer of natural gas mixtures, amongst many other uses. The refrigeration industry has been using the multi-fluid modeling approach for many years and will likely do so for many years to come.

Pure fluid equations of state. In this framework, the equation of state for a pure component is given in terms of the Helmholtz energy, in terms of the Massieu potential, given by $\alpha=$ $a /(R T)$. Thermodynamic properties are obtained from combinations of the Helmholtz energy and its derivatives. For instance, the pressure is obtained from $p=-\partial a / \partial v$. The total $\alpha$ is given as the sum of ideal-gas (non-interacting) and residual (interacting) contributions. The ideal-gas portion can in principle be obtained from theory for the given molecular species, and the residual contribution is entirely empirical, fit to experimental data as well and constrained to have the desired behavior at extremes in temperature and pressure. For most pure fluids, the residual Helmholtz energy can be expressed generically as

$$
\alpha^{\mathrm{r}}=\sum_{i} n_{i} \delta^{d_{i}} \tau^{t_{i}} \exp \left(-c_{i} \delta^{l_{i}}-\eta_{i}\left(\delta-\varepsilon_{i}\right)^{2}-\beta_{i}\left(\tau-\gamma_{i}\right)^{2}\right)
$$

where $\tau=T_{\text {red }} / T$ and $\delta=\rho / \rho_{\text {red }}$ with $T_{\text {red }}$ and $\rho_{\text {red }}$ being the reducing temperature and reducing density, respectively. All remaining variables are empirical coefficients particular to the given fluid.

Mixture equation of state. The same thermodynamic identities hold for mixtures as for pure fluids. The Helmholtz energy for a mixture is commonly obtained as the sum of a corresponding states contribution and a departure term.

$$
\alpha^{r}=\alpha_{C S}^{r}(\tau, \delta, \bar{x})+\alpha_{d e p}^{r}(\tau, \delta, \bar{x})
$$

in which

$$
\alpha^{r}=\sum_{i=1}^{N} x_{i} \alpha_{o, i}^{r}(\tau, \delta)+\sum_{i=1}^{N} \sum_{j=i+1}^{N} F_{i j} x_{i} x_{j} \alpha_{i j}^{r}(\tau, \delta),
$$

and for mixtures $\tau=T_{\text {red }}(\bar{x}) / T$ and $\delta=\rho / \rho_{\text {red }}(\bar{x})$. The reducing functions are given by 


$$
\begin{aligned}
& T_{\mathrm{r}}(\bar{x})=\sum_{i=1}^{N} \sum_{j=1}^{N} x_{i} x_{j} \beta_{T, i j} \gamma_{T, i j} \frac{x_{i}+x_{j}}{\beta_{T, i j}^{2} x_{i}+x_{j}}\left(T_{\mathrm{c}, i} \cdot T_{\mathrm{c}, j}\right)^{0.5} \\
& \frac{1}{\rho_{\mathrm{r}}(\bar{x})}=\sum_{i=1}^{N} \sum_{j=1}^{N} x_{i} x_{j} \beta_{v, i j} \gamma_{v, i j} \frac{x_{i}+x_{j}}{\beta_{v, i j}^{2} x_{i}+x_{j}} \frac{1}{8}\left(\frac{1}{\rho_{\mathrm{c}, i}^{1 / 3}}+\frac{1}{\rho_{\mathrm{c}, j}^{1 / 3}}\right)^{3} .
\end{aligned}
$$

Thus the reducing functions have four adjustable parameters per $i j$ binary pair.

The phase equilibrium pressure is the result of an iterative calculation to equate pressure, temperature, chemical potentials of all species in all phases and amount of substances.

Parameter Optimization. We used the parameter optimization approach described in ${ }^{7}$. The parameters $\beta_{T, i j}$ and $\gamma_{T, i j}$ were obtained for each binary pair individually, leaving the other interaction parameters set to their default values. The experimental data were added to the database of experimental data used in the fitting tool. Then the python script was launched which carried out the optimization, doing a stochastic global optimization over the two interaction parameters with the DEAP software package. The approach used is a classical evolutionary optimization methodology with crossover/mutation, etc. The open-source fitting routines are at https://github.com/ianhbell/binfit. This mixture behavior is ideal enough that simpler optimization approaches could have been used, but this implementation has proven to be reliable for fitting interaction parameters, particularly for fitting interaction parameters to outputs of iterative routines that can fail for a variety of different reasons. Failures of iterative routines are handled by adding a large contribution to the cost function. Derivatives are not used in the optimization approach, so the cost function to be minimized need not be differentiable.

Mixture thermal conductivity model. In recent years it has become increasingly clear that the transport properties are strongly connected with the residual entropy (i.e., the entropy of a fluid minus the contribution of the ideal-gas, or zero-density limit). This result was first proposed by Rosenfeld in $1977^{86}$ based on the limited molecular dynamics simulations available at that time. The advent of modern computational resources has meant that Rosenfeld's suggestion has been investigated in greater detail in recent years. The LennardJones 12-6 fluid, one of the most popular molecular model fluids because it includes physically reasonable attraction and repulsion contributions, has recently been shown to follow entropy scaling for viscosity, thermal conductivity, and self-diffusion. ${ }^{87}$ Additional studies have demonstrated that the general framework of modified residual entropy scaling allows for an excellent collapse of the experimental transport data for other systems, including the viscosity of propane ${ }^{88}$, normal alkanes ${ }^{89}$, and refrigerants. ${ }^{90}$

Other researchers ${ }^{91-94}$ have applied an older variant of entropy scaling to refrigerant mixtures, but their overall approach of reducing by the dilute-gas viscosity does not follow the correct theoretical constraints in the liquid phase, which is particularly problematic for small molecules like methane and argon.

The approach of modified residual entropy scaling meets the required theoretical limits in the liquid phase, does not diverge at zero density like the original Rosenfeld scaling approach, 
and also allows for a formulation consistent with highly accurate dilute-gas measurements and modeling.

In this work we follow the modified residual entropy scaling approach. To begin, the residual entropy is defined by

$$
s^{\mathrm{r}} \equiv s(T, \rho)-s^{\mathrm{ig}}(T, \rho)
$$

where $s$ is the entropy per particle and $s^{\text {ig }}$ is the ideal-gas entropy per particle, so the value of the reduced scaled residual entropy is given by $s^{+} \equiv-s^{\mathrm{r}} / k_{B}$ if $s^{\mathrm{r}}$ is on a per-particle basis. The residual entropy $s^{\mathrm{r}}$ is a measure of the structure of the fluid phase. Intermolecular interactions have the impact of reducing the possible microstates, so $s^{\mathrm{r}}$ is generally negative, and therefore $s^{+}$is positive.

The Rosenfeld-scaled (or macroscopically scaled) thermal conductivity is defined based on non-dimensionalizing the thermal conductivity by the appropriate length, time, and energy scales for the liquid phase. This yields the definition

$$
\tilde{\lambda}=\frac{\lambda}{k_{B} \rho_{N}^{2 / 3} \sqrt{k_{B} T / m}}
$$

As the number density $\rho_{N}=\rho N_{A}$ goes to zero at constant temperature, the value of $\tilde{\lambda}$ diverges, but this divergence can be repaired by taking advantage of the result noted by Rosenfeld for inverse-power-law (IPL) fluids of very low but finite density that scaled transport properties are proportional to $\left(s^{+}\right)^{-2 / 3}$, which allows for the definition of a term that breaks the zero-density divergence:

$$
\lambda^{+}=\tilde{\lambda} \times\left(s^{+}\right)^{2 / 3}
$$

In the case of mixtures, a mixing rule must be defined for the effective mass of one particle $m$. In other works, the mass of the heaviest particle has been used ${ }^{95}$. Mass-fraction weighting of the molecular masses of the components has proven to be the most successful scaling approach in this case, slightly better than mole-fraction weighting of the molecular masses. Otherwise, the quantities $\rho_{N}$ and $s^{+}$are obtained from an empirical equation of state. In this case, we have used the updated interaction parameters fitted in this work. 


\section{A.2. Task 2 - Flammability Experiments and Test Data}

First, the experimental data for the ASHRAE Standard 34 E681 test are presented. Following that, the modified Japanese High-Pressure Gas Law test is described and the experimental results in that device are presented. The next section presents the experimental approach for the laminar burning velocity measurements in the 2 - $\mathrm{L}$ chamber, and the experimental results for that test are provided.

\section{A.2.1. ASHRAE Standard 34 E681 Test Data}

Table A.2-1. E681 test results for R-513A, NIST-ternary blend, R-450A, R-515B, and blends of R-1234yf/134a at a molar composition of $(0.26 / 0.74),(0.28 / 0.72)$, and $(0.30 / 0.70)$.

\begin{tabular}{cccccc}
\hline $\begin{array}{c}\text { Volume } \\
\text { Fraction } \\
(\%)\end{array}$ & $\begin{array}{c}\text { Pressure } \\
(\mathbf{m m H g})\end{array}$ & $\begin{array}{c}\text { Air } \\
\text { R.H. } \\
(\%)\end{array}$ & $\begin{array}{c}\text { Flame } \\
\text { Angles } \\
(\text { degrees })\end{array}$ & $\begin{array}{c}\text { Result } \\
(\text { Go/ } / \\
\text { NoGo })\end{array}$ & Comment \\
\hline
\end{tabular}

\begin{tabular}{|c|c|c|c|c|c|}
\hline 6 & 760 & 49.3 & No angle & No Go & Just spark, no flame \\
\hline 7 & 760 & 50.2 & No angle & No Go & $\begin{array}{l}\text { Little flame bubble around the } \\
\text { spark, disappears within 1" }\end{array}$ \\
\hline 8 & 760 & 49.4 & No angle & No Go & $\begin{array}{l}\text { Little flame bubble around the } \\
\text { spark, disappears within 1" }\end{array}$ \\
\hline 9 & 760 & 49.7 & No angle & No Go & $\begin{array}{l}\text { Little flame bubble around the } \\
\text { spark, disappears within 1" }\end{array}$ \\
\hline 10 & 760 & 51.8 & No angle & No Go & $\begin{array}{l}\text { Little flame bubble around the } \\
\text { spark, disappears within 1" }\end{array}$ \\
\hline 11 & 760 & 51.8 & No angle & No Go & $\begin{array}{l}\text { Little flame bubble around the } \\
\text { spark, disappears within 1" }\end{array}$ \\
\hline 12 & 760 & 52.0 & No angle & No Go & $\begin{array}{l}\text { Little flame bubble around the } \\
\text { spark, disappears within 1" }\end{array}$ \\
\hline 13 & 760 & 51.5 & No angle & No Go & $\begin{array}{l}\text { Little flame bubble around the } \\
\text { spark, disappears within 1" }\end{array}$ \\
\hline
\end{tabular}

\section{R-1234yf/134a (74.0/26.0 by vol\%) flammability at $60^{\circ} \mathrm{C}$}

\begin{tabular}{lccccl}
\hline 6 & 760 & 51.1 & No angle & No Go & Candle flame \\
7 & 760 & 51.1 & No angle & No Go & Flame didn't reach the top \\
7 & 760 & 51.3 & No angle & No Go & Flame didn't reach the top \\
8 & 760 & 52 & 86 & No Go & \\
8 & 760 & 50.3 & 95 & Go & \\
8 & 760 & 49.9 & 100 & Go & Go \\
9 & 760 & 51.6 & 92 & No Go & \\
9 & 760 & 51.2 & 85 & No Go &
\end{tabular}




\begin{tabular}{|c|c|c|c|c|c|}
\hline 10 & 760 & 51.7 & 88 & No Go & \\
\hline 10 & 760 & 51.5 & 91 & Go & \\
\hline 10 & 760 & 51.2 & 98 & Go & \\
\hline 11 & 760 & 51.8 & 100 & Go & \\
\hline 11 & 760 & 51.5 & 81 & No Go & \\
\hline 11 & 760 & 50.6 & 82 & No Go & \\
\hline 12 & 760 & 51.7 & 88 & No Go & \\
\hline 12 & 760 & 50.7 & 88 & No Go & \\
\hline 13 & 760 & 50.6 & No angle & No Go & \\
\hline \multicolumn{6}{|c|}{$\mathrm{R}-1234 \mathrm{yf} / 134 \mathrm{a}\left(\mathbf{7 2 . 0 / 2 8 . 0}\right.$ by vol\%) flammability at $60^{\circ} \mathrm{C}$} \\
\hline 6 & 760 & 50.4 & No angle & No Go & \\
\hline 7 & 760 & 50.3 & No angle & No Go & Flame didn't reach the top \\
\hline 8 & 760 & 51.9 & 90 & No Go & \\
\hline 8 & 760 & 48.9 & 95 & Go & \\
\hline 8 & 760 & 49.8 & 88 & Go & \\
\hline 9 & 760 & 52.0 & 91 & Go & \\
\hline 9 & 760 & 50.5 & 91 & Go & \\
\hline 9 & 760 & 49.9 & 95 & Go & \\
\hline 10 & 760 & 51.9 & 100 & Go & \\
\hline 10 & 760 & 48.9 & 90 & No Go & \\
\hline 10 & 760 & 50.2 & 92 & Go & \\
\hline 11 & 760 & 48.9 & 95 & Go & \\
\hline 11 & 760 & 51.9 & 97 & Go & \\
\hline 11 & 760 & 51.3 & 95 & Go & \\
\hline 12 & 760 & 51.4 & No angle & No Go & Flame fell apart \\
\hline 13 & 760 & 51.8 & No angle & No Go & $\begin{array}{l}\text { Flame fell apart at the top of the } \\
\text { flask }\end{array}$ \\
\hline
\end{tabular}

R-1234yf/134a (70.0/30.0 by vol\%) flammability at $60^{\circ} \mathrm{C}$

$\begin{array}{cccccl}6.1 & 760 & 49.7 & \text { No angle } & \text { No Go } & \begin{array}{l}\text { Spark only } \\ 7.0\end{array} \\ 8.0 & 760 & 50.4 & \text { No angle } & \text { No Go } & \begin{array}{l}\text { Flame didn't reach the top } \\ \text { No Go }\end{array} \\ & & 52.0 & 55 & \begin{array}{l}\text { Practically no angle, flame } \\ \text { didn't reach the top, it was } \\ \text { around 55 deg wide before it } \\ \text { disappeared }\end{array} \\ 8.0 & 760 & 50.6 & 80 & \text { No Go } & \\ 8.0 & 760 & 49.7 & 80 & \text { No Go } & \\ 8.9 & 760 & 50.8 & 91 & \text { Go } & \end{array}$




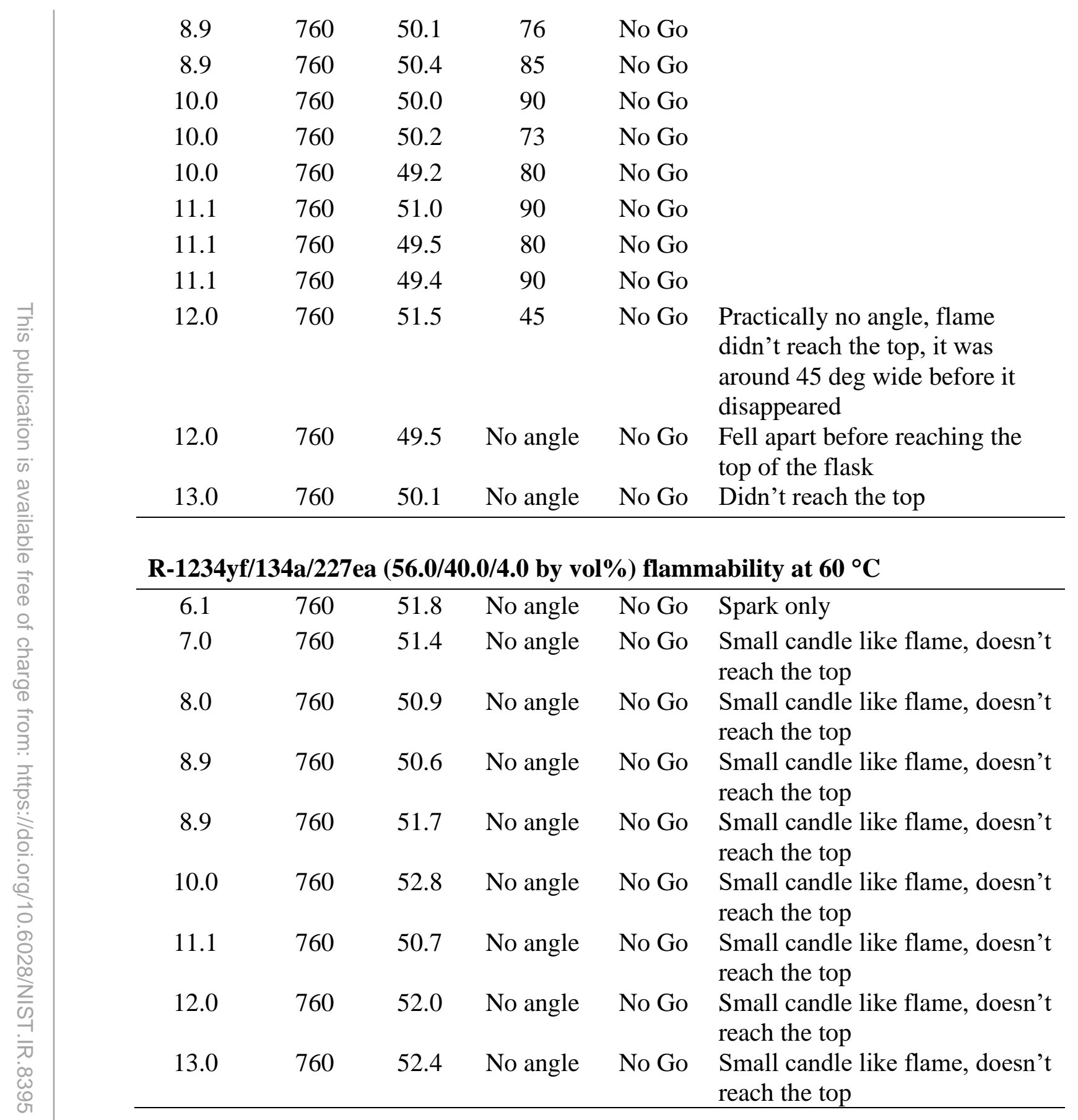




\section{A.2.2. Japanese High-Pressure Gas Law Test}

JHPGL Experiment Description. The constant-volume combustion device ${ }^{29}$ is based on the Japanese High Pressure Gas Law (JHPGL), and is similar to the design of Takizawa ${ }^{96}$, Shebeko ${ }^{97}$, and others. The experimental 2-L apparatus is shown in Figure A.2-1 while the plumbing schematic is shown in Figure A.2-1. The chamber consists of a stainless steel (316) sphere with an inner diameter of $15.24 \mathrm{~cm}$, a volume of $1.85 \mathrm{~L}$, and wall thickness of $2.54 \mathrm{~cm}$; the vessel has nine tapped openings for gas inlet and outlet ports, and various transducers. Gases are introduced via the partial pressure mixing technique, and ignition is provided by a fused platinum wire (Alfa Aesar 43014-BY). Rather than basing flammability on a thermocouple temperature rise as specified in the JHPGL, a dynamic pressure transducer was added, allowing a pressure-based criterion.

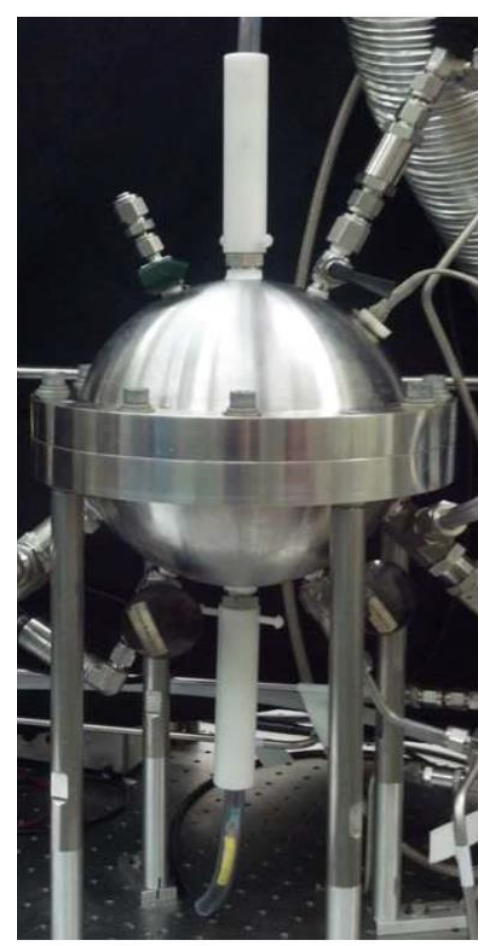

Figure A.2-1. Spherical 2-L chamber for JHPGL tests and constant volume method laminar burning velocity measurements.

Component partial pressures are determined with an absolute pressure transducer (Omega PX811-030AV, 0 to $206.8 \mathrm{kPa}$ range) with a claimed accuracy of $\pm 0.1 \%$, coupled to a digital strain gage readout (Omega* DP80). These are periodically calibrated against a Baratron 627D (claimed accuracy of $0.12 \%$ ) and a Wallace \& Tiernan 1500 (claimed accuracy of $0.066 \%$ ) pressure gage. Uncertainty in the pressure reading is estimated to be $2 \%$ of the reading. The refrigerants were HFC-134a, HFO-1234yf, R-513A, HFO-1234ze(E), and R515B. 


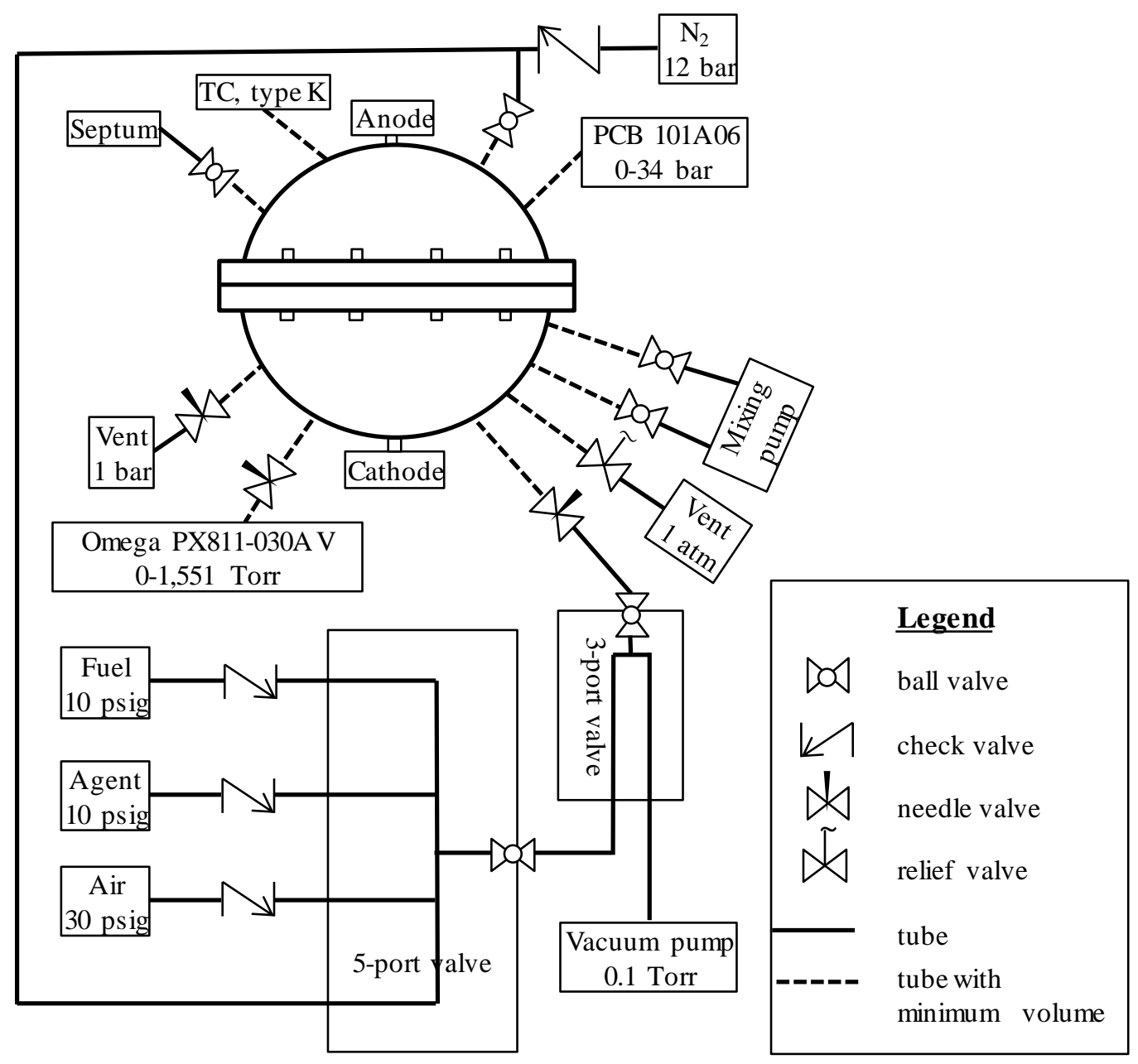

Figure A.2-2. Plumbing schematic diagram for 2-L chamber.

The air was house compressed air (filtered and dried) which is additionally cleaned by passing it through an $0.01 \mu \mathrm{m}$ filter, a carbon filter, and a desiccant bed to remove small aerosols, organic vapors, and water vapor. The relative humidity of the dry shop air was measured in previous work to be less than $2 \%$ at $22^{\circ} \mathrm{C}$. When desired, water was added as a reactant using a $50 \mu \mathrm{L}$ syringe (Millipore Sigma, Model \# 22269-U) through a septum (subsequently closed-off via a ball valve) and allowed to vaporize. After all gases were added they were mixed by a stainless-steel bellows pump in an external loop (evacuated between tests) and allowed to settle for 5 minutes before ignition. The initial temperature of the vessel, measured by a fine type-K thermocouple on the chamber exterior wall, was typically $(26.2 \pm 0.6){ }^{\circ} \mathrm{C}$.

The igniter configuration was modified slightly from that recommended in the JHPGL. Rather than the igniter leads entering the chamber from two locations at right angles to each other, two parallel copper leads (57 mm long, $1 \mathrm{~mm}$ diameter) were used separated by $4 \mathrm{~mm}$ and with Teflon insulation around the copper wires in select locations. Crimp-on connections (Digi-Key A2161-ND and A34501-ND) were used between the copper and platinum wires instead of welds. Hence, the igniter could be inserted through a single $6.35 \mathrm{~mm}$ inner 
diameter orifice, with easily replaceable fusible wire. A variable transformer AC power supply (Powerstat, model 30N116C) provided $100 \mathrm{VAC}$ to the igniter, and its manual switchcontrolled ignition. An isolation transformer (Hammond Manufacturing, model 171G, 1000 VA) was used to separate the ignition system from the house electrical supply. This provided a more consistent energy to the igniter and prevented blowing the $10 \mathrm{~A}$ fuse in the variable transformer. The platinum wire $(20 \mathrm{~mm}$ long, $0.3 \mathrm{~mm}$ diameter) melted and ruptured violently during each ignition and was replaced.

A data acquisition system (DAS, National Instruments models NI USB-6259 and NI SCC-68, with LabVIEW VI) connected to a personal computer (Dell OptiPlex 7060) recorded the dynamic pressure during each experiment. A dynamic pressure sensor (PCB Piezotronics, model 101 A06) with a rise time of $1.5 \mu \mathrm{sec}$, a range of $3450 \mathrm{kPa}$, and reported accuracy of $0.07 \mathrm{kPa}$, measured the pressure rise in the chamber. The DAS collected data for $60 \mathrm{~s}$ at $100 \mathrm{~Hz}$.

Product gases were removed from the chamber at the end of each test to prevent contamination for the subsequent test. Gaseous nitrogen was introduced to the chamber soon after the ignition: 1.) to quickly purge the corrosive acid gases present for some experiments, and 2.) to reduce the temperature of the product gases (to protect components). After ignition, followed by a $10 \mathrm{~s}$ delay, gaseous nitrogen at 11 bar pressurized the chamber for $5 \mathrm{~s}$ after which the exhaust valve was opened, and $\mathrm{N}_{2}$ flowed continuously for $10 \mathrm{sec}$. Following the nitrogen purge, clean and dried shop air flushed the chamber for ten minutes, the chamber was evacuated and maintained for 2 minutes, followed by dry air purge for 2 minutes (with these last two steps repeated).

\section{Japanese High-Pressure Gas Law Test Data}

Table A.2-2. Japanese High-Pressure Gas Law test results obtained at NIST for: R-513A, NIST-ternary blend, R-450A, R-515B, and blends of R-1234yf/134a, all with dry or $50 \%$ r.h. air as listed. 


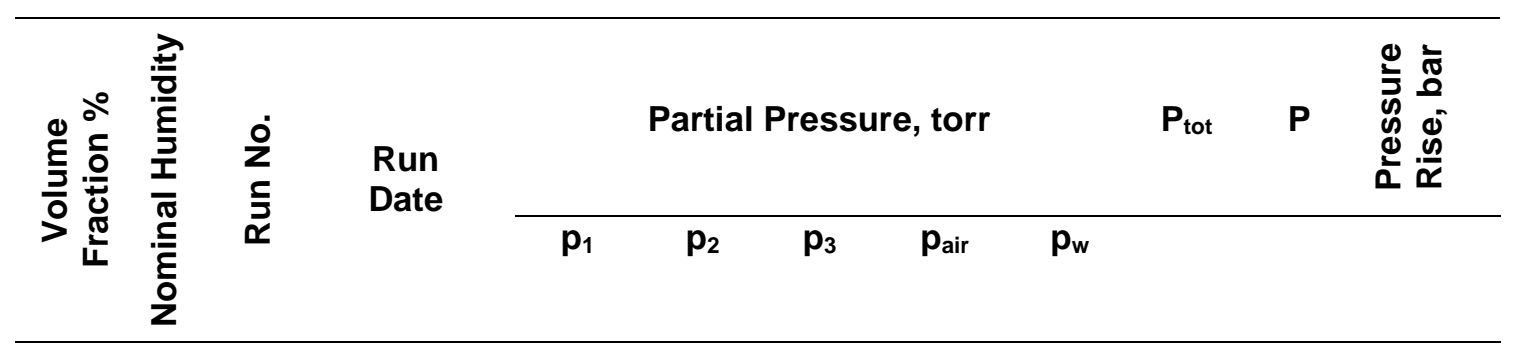

\begin{tabular}{|c|c|c|c|c|c|c|c|c|c|c|c|}
\hline & & & & & & & & & & & \\
\hline 7.0 & Dry & 16 & $2020-10-22$ & 26.2 & 29.7 & $N / A$ & 742.4 & $\mathrm{~N} / \mathrm{A}$ & 798.2 & 759.8 & 0.003 \\
\hline 8.0 & & 17 & $2020-10-22$ & 29.9 & 33.9 & & 734.5 & & 798.3 & 759.9 & 0.097 \\
\hline 9.3 & & 1 & $2020-10-20$ & 34.6 & 39.3 & & 724.4 & & 798.2 & 760.0 & 0.080 \\
\hline 10.0 & & 2 & $2020-10-20$ & 37.4 & 42.4 & & 718.6 & & 798.3 & 759.9 & 0.202 \\
\hline 10.5 & & 3 & $2020-10-20$ & 39.3 & 44.5 & & 714.5 & & 798.2 & 759.7 & 0.178 \\
\hline 11.0 & & 4 & $2020-10-20$ & 41.2 & 46.7 & & 710.5 & & 798.3 & 760.0 & 0.191 \\
\hline 11.0 & & 18 & $2020-10-22$ & 41.1 & 46.7 & & 710.5 & & 798.2 & 759.8 & 0.214 \\
\hline 11.3 & & 10 & $2020-10-21$ & 42.1 & 47.7 & & 708.7 & & 798.4 & 760.3 & 0.253 \\
\hline 11.5 & & 11 & $2020-10-21$ & 43.0 & 48.8 & & 706.6 & & 798.4 & 760.0 & 0.242 \\
\hline 12.0 & & 12 & $2020-10-21$ & 44.9 & 50.9 & & 702.8 & & 798.4 & 760.0 & 0.255 \\
\hline 12.5 & & 7 & $2020-10-21$ & 46.7 & 53.0 & & 698.7 & & 798.4 & 760.1 & 0.178 \\
\hline 13.0 & & 8 & 2020 & 48.6 & 55 & & 694.6 & & 798.3 & 760.1 & 0.209 \\
\hline 14.0 & & 9 & 2020 & 52.4 & 59.4 & & 686.7 & & 3.3 & 760.1 & 0.239 \\
\hline 15.0 & & 13 & $0-22$ & 56.1 & 63 & & 67 & & & .7 & 0. \\
\hline 16.0 & & 15 & 202 & 59.9 & 67 & & 67 & & & 0 & 0. \\
\hline 17.0 & & 14 & -22 & 63.6 & 72 & & 66 & & & & 0.004 \\
\hline 11.5 & & 5 & 20 & 43.0 & 48 & & 6 & & & & 0. \\
\hline 12.0 & & 6 & -21 & 44.9 & 50.9 & & 702.7 & & .5 & 76 & 0.004 \\
\hline 5.0 & 42 & 10 & 202 & 18 & 21.2 & $\mathrm{~N}$ & 747.7 & & 79 & 759.8 & 0.027 \\
\hline 6.0 & 52 & 9 & 2020 & 22.4 & 25.4 & & 739.7 & 10 & 798.3 & 759.2 & 0.061 \\
\hline 7.0 & 46 & 8 & 2020 & 26.2 & 29.7 & & 732.1 & 10 & 798.2 & 759.4 & 0.077 \\
\hline 8.0 & 55 & 7 & $1-12$ & 29.9 & 33.9 & & 724.4 & 10 & 798.3 & 759.5 & 0.096 \\
\hline 10.0 & 50 & 1 & $1-10$ & 37.4 & 42.4 & & 707.8 & 9 & 798.3 & 9.5 & 306 \\
\hline 11.0 & 58 & 5 & 202 & 41.1 & 46.7 & & .1 & 8 & 3.4 & 9.8 & 0.446 \\
\hline 12.0 & 58 & 2 & 202 & 44.9 & 50.9 & & .0 & & 8.3 & & 0.474 \\
\hline 13.0 & 55 & 6 & 202 & 48.6 & 55.1 & & & & 8.1 & 59.2 & 0.330 \\
\hline 14.0 & 45 & 3 & 202 & 52.3 & 59.4 & & 676.3 & 10.4 & 798.2 & 759.4 & 0.173 \\
\hline 16.0 & 53 & 4 & 202 & 59.8 & 67.9 & & 660.1 & 11.0 & 798.5 & 759.7 & 0.095 \\
\hline 18.0 & 53 & 11 & $2020-11-12$ & 67.3 & 76.3 & & 644.4 & 10.5 & 798.3 & 759.4 & 0.035 \\
\hline 19.0 & 58 & 12 & $2020-11-12$ & 71.1 & 80.6 & & 636.3 & 10.6 & 798.4 & 759.7 & 0.002 \\
\hline
\end{tabular}

R-450A (R134a/1234ze(E)) (0.447/0.553, mole fraction)

$\begin{array}{cccccccccccc}4.0 & \text { Dry } & 13 & 2020-11-25 & 14.3 & 17.7 & \text { N/A } & 766.7 & \text { N/A } & 798.2 & 759.9 & 0.044 \\ 6.0 & 10 & 2020-11-25 & 21.4 & 26.4 & & 750.8 & & 798.2 & 760.1 & 0.027 \\ 8.0 & 6 & 2020-11-24 & 28.6 & 35.3 & & 734.7 & & 798.2 & 759.6 & 0.046 \\ 10.0 & 1 & 2020-11-24 & 35.7 & 44.2 & & 718.8 & & 798.4 & 760.2 & 0.141 \\ 10.9 & 11 & 2020-11-25 & 39.2 & 48.5 & & 715.6 & & 803.0 & 760.3 & 0.061 \\ 12.0 & 3 & 2020-11-24 & 42.8 & 53.0 & & 702.9 & & 798.2 & 760.2 & 0.258 \\ 13.0 & 4 & 2020-11-24 & 46.4 & 57.4 & 695.0 & & 798.3 & 760.0 & 0.147 \\ 15.0 & 7 & 2020-11-24 & 53.5 & 66.2 & 679.0 & & 798.2 & 759.9 & 0.164 \\ 16.0 & 12 & 2020-11-25 & 57.1 & 70.6 & 671.0 & & 798.2 & 759.9 & 0.137 \\ 18.0 & 8 & 2020-11-25 & 64.2 & 79.4 & 655.0 & & 798.2 & 759.6 & 0.030 \\ 20.0 & 9 & 2020-11-25 & 71.4 & 88.3 & 639.0 & & 798.2 & 760.0 & 0.028\end{array}$




\begin{tabular}{|c|c|c|c|c|c|c|c|c|c|c|c|}
\hline 22.0 & & 14 & $2020-11-25$ & 78.5 & 97.1 & & 623.3 & & 798.4 & 759.9 & 0.014 \\
\hline 11.0 & & 2 & $2020-11-24$ & 39.3 & 48.6 & & 710.8 & & 798.2 & 760.1 & 0.004 \\
\hline 11.0 & & 5 & $2020-11-24$ & 39.2 & 48.5 & & 710.7 & & 798.1 & 759.4 & 0.003 \\
\hline 5.0 & 53 & 14 & $2020-11-23$ & 17.8 & 22.1 & N/A & 747.7 & 11.1 & 798.3 & 759.5 & 0.012 \\
\hline 6.0 & 53 & 5 & $2020-11-20$ & 21.3 & 26.4 & & 739.6 & 11.1 & 798.2 & 760.2 & 0.066 \\
\hline 7.0 & 56 & 6 & $2020-11-20$ & 25.0 & 30.9 & & 731.2 & 11.6 & 798.4 & 760.1 & 0.095 \\
\hline 8.0 & 42 & 1 & $2020-11-19$ & 28.5 & 35.4 & & 726.2 & 8.5 & 798.3 & 759.7 & 0.128 \\
\hline 9.0 & 56 & 7 & $2020-11-20$ & 32.1 & 39.8 & & 715.6 & 11.2 & 798.3 & 760.0 & 0.186 \\
\hline 10.0 & 53 & 2 & $2020-11-19$ & 35.6 & 44.2 & & 708.4 & 10.5 & 798.4 & 759.6 & 0.262 \\
\hline 11.0 & 55 & 8 & $2020-11-20$ & 39.2 & 48.6 & & 700.3 & 10.8 & 798.4 & 759.8 & 0.124 \\
\hline 12.5 & 58 & 13 & $2020-11-23$ & 44.6 & 55.1 & & 687.7 & 11.2 & 798.3 & 759.3 & 0.185 \\
\hline 13.0 & 57 & 9 & $2020-11-20$ & 46.4 & 57.3 & & 683.9 & 10.9 & 798.2 & 759.7 & 0.161 \\
\hline 14.0 & 56 & 3 & $2020-11-19$ & 50.0 & 61.8 & & 676.1 & 10.7 & 798.3 & 759.5 & 0.205 \\
\hline 15.0 & 58 & 10 & $2020-11-20$ & 53.5 & 66.2 & & 668.0 & 10.8 & 798.2 & 759.8 & 0.156 \\
\hline 16.0 & 57 & 4 & $2020-11-19$ & 57.1 & 70.6 & & 660.4 & 10.7 & 798.3 & 759.5 & 0.019 \\
\hline 17.0 & 59 & 15 & $2020-11-23$ & 60.9 & 74.9 & & 652.1 & 10.9 & 798.4 & 759.4 & 0.083 \\
\hline 19.0 & 60 & 16 & $2020-11-23$ & 67.8 & 83.9 & & 636.2 & 10.8 & 798.3 & 759.6 & 0.060 \\
\hline 11.0 & 50 & 11 & $2020-11-20$ & 39.2 & 48.6 & & 700.9 & 9.9 & 798.3 & 759.9 & 0.083 \\
\hline 13.0 & 55 & 12 & $2020-11-23$ & 46.3 & 57.6 & & 684.2 & 10.5 & 798.3 & 759.7 & 0.141 \\
\hline
\end{tabular}

\begin{tabular}{cccccccccccc}
\multicolumn{1}{l}{ NIST Ternary Blend (R134a/1234yf/1234ze(E) } & \multicolumn{2}{l}{$\mathbf{0 . 5 2 / 0 . 3 2 / 0 . 1 6}$, mole fraction) } \\
10.5 & Dry & 7 & $2020-11-04$ & 311.9 & 192.1 & 96.0 & 714.5 & N/A & 798.4 & 760.2 & 0.003 \\
11.0 & & 5 & $2020-11-04$ & 312.0 & 192.0 & 96.2 & 710.6 & & 798.5 & 760.0 & 0.003 \\
11.3 & & 8 & $2020-11-04$ & 312.0 & 192.1 & 95.9 & 708.5 & & 798.3 & 760.2 & 0.210 \\
11.4 & 1 & $2020-11-03$ & 311.8 & 192.1 & 95.9 & 711.4 & & 803.2 & 764.8 & 0.211 \\
12.0 & & 6 & $2020-11-04$ & 312.0 & 192.1 & 95.9 & 702.6 & & 798.4 & 759.9 & 0.203 \\
12.5 & 2 & $2020-11-03$ & 312.0 & 191.9 & 96.0 & 698.4 & & 798.3 & 760.0 & 0.123 \\
13.5 & & 3 & $2020-11-03$ & 312.0 & 192.0 & 95.9 & 690.5 & & 798.4 & 760.0 & 0.000 \\
14.0 & & 4 & $2020-11-04$ & 312.0 & 191.9 & 96.0 & 686.6 & & 798.4 & 760.1 & 0.001 \\
\hline 8.0 & 54 & 5 & $2020-11-06$ & 33.2 & 20.5 & 10.2 & 723.6 & 11.0 & 798.3 & 759.3 & 0.002 \\
9.0 & 53 & 4 & $2020-11-06$ & 37.3 & 23.0 & 11.5 & 716.0 & 10.7 & 798.3 & 759.7 & 0.138 \\
10.0 & 55 & 7 & $2020-11-09$ & 41.5 & 25.5 & 12.7 & 707.9 & 11.0 & 798.4 & 758.7 & 0.192 \\
11.0 & 52 & 1 & $2020-11-06$ & 45.3 & 28.2 & 14.0 & 700.9 & 10.3 & 798.4 & 759.8 & 0.406 \\
12.0 & 50 & 9 & $2020-11-09$ & 49.8 & 30.6 & 15.3 & 691.9 & 10.9 & 798.3 & 759.6 & 0.451 \\
13.0 & 50 & 2 & $2020-11-06$ & 53.9 & 33.2 & 16.6 & 685.2 & 9.6 & 798.3 & 759.8 & 0.234 \\
15.0 & 57 & 3 & $2020-11-06$ & 62.2 & 38.3 & 19.1 & 668.2 & 10.7 & 798.3 & 759.4 & 0.131 \\
16.0 & 63 & 6 & $2020-11-06$ & 66.4 & 40.9 & 20.5 & 659.2 & 11.6 & 798.3 & 759.6 & 0.120 \\
17.0 & 57 & 10 & $2020-11-09$ & 70.5 & 43.5 & 21.7 & 652.5 & 10.5 & 798.3 & 759.8 & 0.083 \\
18.0 & 60 & 11 & $2020-11-09$ & 74.7 & 46.0 & 22.9 & 644.0 & 10.9 & 798.4 & 759.8 & 0.048 \\
12.0 & 57 & 8 & $2020-11-09$ & 49.8 & 30.6 & 15.4 & 691.8 & 11.0 & 798.4 & 760.2 & 0.023 \\
\hline
\end{tabular}

R-515B (R1234ze(E)/R227ea) (0.939/0.061, mole fraction)

$\begin{array}{cccccccccccc}4.0 & \text { Dry } & 15 & 2020-11-16 & 2.0 & 30.0 & \text { N/A } & 766.6 & \text { N/A } & 798.1 & 759.7 & 0.005 \\ 5.0 & 14 & 2020-11-16 & 2.5 & 37.5 & & 758.8 & & 798.4 & 759.9 & 0.072 \\ 6.0 & 13 & 2020-11-16 & 2.9 & 45.0 & & 750.7 & & 798.3 & 759.8 & 0.101 \\ 7.0 & 7 & 2020-11-13 & 3.4 & 52.5 & & 742.7 & & 798.3 & 759.0 & 0.053 \\ 8.0 & 6 & 2020-11-13 & 3.9 & 60.0 & & 734.7 & & 798.3 & 759.6 & 0.415 \\ 9.0 & 9 & 2020-11-13 & 4.4 & 67.5 & 726.7 & & 798.3 & 759.6 & 0.507 \\ 9.6 & 1 & 2020-11-13 & 4.7 & 72.0 & 721.6 & & 798.1 & 759.7 & 1.031 \\ 9.6 & 17 & 2020-11-18 & 4.7 & 71.9 & 722.2 & & 798.4 & 760.3 & 1.564 \\ 10.0 & 8 & 2020-11-13 & 4.9 & 74.9 & 718.7 & & 798.2 & 759.8 & 1.221 \\ 10.0 & 16 & 2020-11-18 & 4.9 & 74.9 & 718.9 & & 798.3 & 760.4 & 0.852 \\ 11.0 & 2 & 2020-11-13 & 5.4 & 82.4 & 710.8 & & 798.4 & 760.0 & 0.400\end{array}$




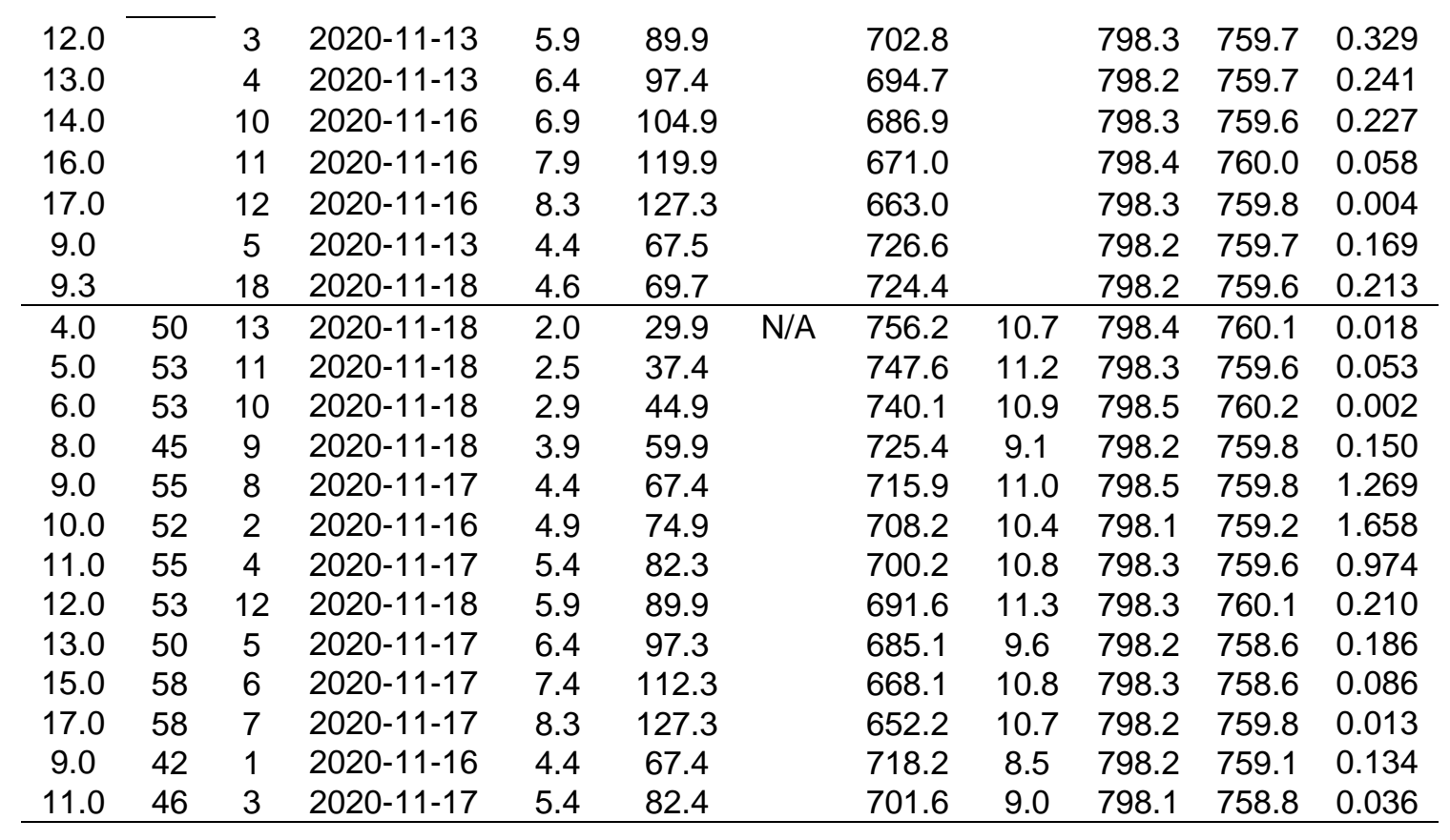

\begin{tabular}{cccccccccccc}
\multicolumn{2}{c}{ R134a/1234yf (0/1.0, mole fraction) } & & & & & & \\
7.0 & Dry & 1 & $2020-12-14$ & 56.0 & N/A & N/A & 743.9 & N/A & 799.1 & 760.5 & 0.060 \\
8.0 & & 2 & $2020-12-14$ & 64.0 & & & 735.2 & & 798.4 & 759.4 & 1.462 \\
8.5 & 8 & $2020-12-15$ & 67.8 & & & 731.2 & & 798.3 & 760.1 & 2.666 \\
9.0 & 3 & $2020-12-14$ & 72.0 & & & 727.2 & & 798.4 & 760.0 & 3.667 \\
10.0 & 4 & $2020-12-14$ & 79.8 & & & 719.3 & & 798.3 & 759.7 & 1.981 \\
11.0 & 5 & $2020-12-14$ & 87.8 & & & 713.0 & & 800.0 & 760.4 & 0.815 \\
12.0 & 6 & $2020-12-14$ & 95.8 & & & 703.5 & & 798.4 & 759.6 & 0.006 \\
13.0 & 7 & $2020-12-14$ & 103.8 & & & 695.3 & & 798.3 & 759.7 & 0.183 \\
\hline 8.0 & 44 & 4 & $2021-01-12$ & 63.8 & N/A & N/A & 727.0 & 9.0 & 798.5 & 759.6 & 2.81 \\
9.0 & 45 & 3 & $2021-01-12$ & 71.8 & & & 718.5 & 9.1 & 798.2 & 759.5 & 4.61 \\
10.0 & 45 & 5 & $2021-01-12$ & 79.7 & & & 711.4 & 9.1 & 798.8 & 759.9 & 1.87 \\
9.0 & 47 & 1 & $2021-01-12$ & 71.8 & & & 718.2 & 9.5 & 798.2 & 759.9 & 2.55 \\
8.9 & 46 & 2 & $2021-01-12$ & 71.8 & & & 723.0 & 9.4 & 802.9 & 760.2 & 2.57 \\
\hline
\end{tabular}

\begin{tabular}{|c|c|c|c|c|c|c|c|c|c|c|c|}
\hline \multicolumn{12}{|c|}{$\mathrm{R} 134 \mathrm{a} / 1234 y f(0.2 / 0.8$, mole fraction) } \\
\hline 7.0 & Dry & 8 & $2020-12-22$ & 11.2 & 44.7 & $\mathrm{~N} / \mathrm{A}$ & 743.2 & $\mathrm{~N} / \mathrm{A}$ & 798.2 & 759.9 & 0.089 \\
\hline 8.0 & & 1 & $2020-12-15$ & 12.9 & 50.9 & & 735.3 & & 798.3 & 759.9 & 0.494 \\
\hline 9.0 & & 9 & $2020-12-22$ & 14.4 & 57.6 & & 727.3 & & 798.4 & 760.0 & 1.540 \\
\hline 10.0 & & 11 & $2020-12-22$ & 16.1 & 63.8 & & 719.4 & & 798.2 & 759.7 & 0.466 \\
\hline 11.0 & & 5 & $2020-12-18$ & 17.5 & 70.4 & & 711.2 & & 798.4 & 759.9 & 0.678 \\
\hline 12.0 & & 6 & $2020-12-18$ & 19.2 & 76.6 & & 703.3 & & 798.3 & 759.8 & 0.006 \\
\hline 9.0 & & 7 & $2020-12-18$ & 14.4 & 57.4 & & 727.3 & & 798.3 & 760.2 & 0.239 \\
\hline 9.0 & & 2 & $2020-12-15$ & 14.4 & 57.5 & & 727.2 & & 798.3 & 759.6 & 0.013 \\
\hline 10.0 & & 3 & $2020-12-15$ & 16.0 & 63.8 & & 719.4 & & 798.4 & 760.1 & 0.990 \\
\hline 11.0 & & 4 & $2020-12-15$ & 17.6 & 70.2 & & 711.5 & & 798.4 & 760.1 & 0.223 \\
\hline 10.0 & & 10 & $2020-12-22$ & 16.0 & 63.8 & & 719.5 & & 798.3 & 760.0 & 0.071 \\
\hline 8.0 & 47 & 3 & 2021-01-06 & 12.7 & 51.2 & $\mathrm{~N} / \mathrm{A}$ & 726.1 & 9.5 & 798.3 & 759.4 & 1.271 \\
\hline 9.0 & 47 & 1 & 2021-01-05 & 14.3 & 57.4 & & 718.3 & 9.4 & 798.2 & 759.2 & 1.987 \\
\hline 10.0 & 46 & 2 & 2021-01-06 & 15.9 & 63.9 & & 710.4 & 9.2 & 798.3 & 759.7 & 0.795 \\
\hline
\end{tabular}




\begin{tabular}{|c|c|c|c|c|c|c|c|c|c|c|c|}
\hline \multicolumn{12}{|c|}{ R134a/1234yf (0.3/0.7, mole fraction) } \\
\hline 8.0 & Dry & 5 & 2021-01-08 & 19.2 & 44.7 & $\mathrm{~N} / \mathrm{A}$ & 735.8 & $\mathrm{~N} / \mathrm{A}$ & 798.4 & 759.9 & 0.087 \\
\hline 9.0 & & 3 & 2021-01-08 & 21.6 & 50.3 & & 730.9 & & 801.4 & 760.1 & 0.787 \\
\hline 10.0 & & 4 & 2021-01-08 & 24.0 & 55.8 & & 719.8 & & 798.3 & 760.0 & 0.780 \\
\hline 11.0 & & 2 & 2021-01-08 & 26.4 & 61.5 & & 711.9 & & 798.4 & 759.9 & 0.203 \\
\hline 10.0 & & 1 & 2021-01-08 & 24.0 & 55.8 & & 719.9 & & 798.4 & 760.2 & 0.524 \\
\hline 9.0 & 46 & 5 & 2021-01-06 & 21.5 & 50.3 & $\mathrm{~N} / \mathrm{A}$ & 718.4 & 9.3 & 798.3 & 759.5 & 0.003 \\
\hline 10.0 & 51 & 4 & 2021-01-05 & 23.9 & 55.9 & & 709.7 & 10.1 & 798.3 & 759.5 & 1.271 \\
\hline 11.0 & 53 & 3 & 2021-01-05 & 26.2 & 61.7 & & 701.0 & 10.5 & 798.2 & 759.2 & 1.047 \\
\hline 12.0 & 43 & 1 & 2021-01-05 & 28.6 & 67.1 & & 695.3 & 8.4 & 798.2 & 759.3 & 0.592 \\
\hline 11.0 & 47 & 2 & 2021-01-05 & 26.2 & 61.5 & & 702.5 & 9.3 & 798.2 & 759.5 & 0.310 \\
\hline
\end{tabular}

\begin{tabular}{cccccccccccc}
\multicolumn{1}{l}{$\mathbf{R} 134 a / 1234 y \mathbf{~ ( 0 . 4 / 0 . 6 , ~ m o l e ~ f r a c t i o n ) ~}$} & & & & & & & \\
9.0 & Dry & 5 & $2021-01-11$ & 28.8 & 43.2 & N/A & 727.6 & N/A & 798.3 & 760.1 & 0.090 \\
10.0 & & 2 & $2021-01-11$ & 31.9 & 48.0 & & 719.8 & & 798.4 & 760.2 & 0.239 \\
11.0 & 4 & $2021-01-11$ & 35.2 & 52.6 & & 711.8 & & 798.4 & 760.2 & 0.119 \\
11.0 & 3 & $2021-01-11$ & 35.2 & 52.7 & & 711.9 & & 798.4 & 760.2 & 0.067 \\
11.0 & 1 & $2021-01-11$ & 35.2 & 52.7 & & 711.9 & & 798.5 & 760.4 & 0.024 \\
\hline 10.0 & 51 & 2 & $2021-01-06$ & 31.9 & 47.9 & N/A & 709.5 & 10.2 & 798.2 & 758.5 & 0.865 \\
11.0 & 53 & 1 & $2021-01-06$ & 35.1 & 52.7 & & 701.4 & 10.4 & 798.4 & 759.5 & 0.936 \\
12.0 & 51 & 3 & $2021-01-06$ & 38.4 & 57.4 & & 693.7 & 10.0 & 798.2 & 759.5 & 0.783 \\
\hline
\end{tabular}

\begin{tabular}{|c|c|c|c|c|c|c|c|c|c|c|c|}
\hline \multicolumn{12}{|c|}{$\mathrm{R} 134 \mathrm{a} / 1234 \mathrm{yf}$ (0.5/0.5, mole fraction) } \\
\hline 7.0 & Dry & 13 & $2020-12-23$ & $28.0^{\prime}$ & 27.9 & $\mathrm{~N} / \mathrm{A}$ & 743.4 & $\mathrm{~N} / \mathrm{A}$ & 798.4 & 760.1 & 0.067 \\
\hline 8.0 & & 10 & 2020-12-07 & 32.0 & 31.9 & & 735.1 & & 798.3 & 759.4 & 0.114 \\
\hline 10.0 & & 6 & 2020-12-04 & 40.0 & 40.0 & & 719.2 & & 798.7 & 760.1 & 0.152 \\
\hline 11.0 & & 1 & $2020-11-30$ & 44.0 & 43.9 & & 710.9 & & 798.2 & 759.2 & 0.205 \\
\hline 11.5 & & 3 & $2020-12-01$ & 45.9 & 45.9 & & 707.2 & & 798.5 & 759.7 & 0.261 \\
\hline 12.0 & & 4 & $2020-12-03$ & 47.9 & 47.8 & & 703.0 & & 798.3 & 759.7 & 0.315 \\
\hline 13.0 & & 7 & $2020-12-07$ & 51.9 & 52.2 & & 694.8 & & 798.3 & 759.6 & 0.227 \\
\hline 15.0 & & 8 & $2020-12-07$ & 59.9 & 59.9 & & 679.1 & & 798.3 & 759.3 & 0.146 \\
\hline 17.0 & & 9 & $2020-12-07$ & 67.9 & 67.9 & & 663.3 & & 798.3 & 759.3 & 0.117 \\
\hline 18.0 & & 11 & $2020-12-23$ & 71.9 & 71.8 & & 655.6 & & 798.4 & 760.4 & 0.104 \\
\hline 20.0 & & 12 & $2020-12-23$ & 79.9 & 79.7 & & 639.8 & & 798.4 & 760.3 & 0.032 \\
\hline 10.0 & & 5 & 2020-12-04 & 40.0 & 39.8 & & 719.1 & & 798.3 & 759.6 & 0.026 \\
\hline 11.5 & & 2 & $2020-11-30$ & 45.9 & 45.9 & & 707.1 & & 798.4 & 759.5 & 0.059 \\
\hline
\end{tabular}

R134a/1234yf (0.55/0.45, mole fraction)

\begin{tabular}{cccccccccccc}
9.0 & 46 & 6 & $2021-01-22$ & 39.6 & 32.5 & N/A & 718.6 & 9.2 & 798.3 & 758.9 & 0.065 \\
10.0 & 53 & 5 & $2021-01-22$ & 44.0 & 35.9 & & 709.3 & 10.5 & 798.3 & 759.7 & 0.121 \\
11.0 & 50 & 4 & $2021-01-22$ & 48.3 & 39.5 & & 702.2 & 9.8 & 798.4 & 759.7 & 0.227 \\
12.0 & 58 & 3 & $2021-01-22$ & 52.7 & 43.1 & & 692.9 & 11.3 & 798.4 & 759.7 & 0.271 \\
13.0 & 58 & 11 & $2021-01-25$ & 57.1 & 46.6 & & 685.2 & 11.1 & 798.4 & 759.8 & 0.147 \\
14.0 & 59 & 12 & $2021-01-25$ & 61.5 & 50.3 & & 677.1 & 11.2 & 798.4 & 759.6 & 0.041 \\
15.0 & 59 & 7 & $2021-01-22$ & 65.9 & 53.8 & & 669.1 & 11.2 & 798.4 & 759.7 & 0.172 \\
16.0 & 66 & 8 & $2021-01-25$ & 70.3 & 57.4 & & 659.9 & 12.2 & 798.3 & 759.5 & 0.131 \\
17.0 & 63 & 10 & $2021-01-25$ & 74.7 & 61.0 & & 652.8 & 11.5 & 798.4 & 759.6 & 0.102 \\
18.0 & 59 & 9 & $2021-01-25$ & 79.0 & 64.6 & & 645.8 & 10.6 & 798.4 & 760.0 & 0.008 \\
13.0 & 58 & 1 & $2021-01-21$ & 57.1 & 46.7 & & 684.6 & 11.3 & 798.2 & 758.5 & 0.078 \\
13.4 & 59 & 2 & $2021-01-22$ & 58.7 & 48.1 & 681.5 & 11.3 & 798.3 & 759.3 & 0.007 \\
\hline
\end{tabular}




\begin{tabular}{|c|c|c|c|c|c|c|c|c|c|c|c|}
\hline \multicolumn{12}{|c|}{ R134a/1234yf (0.7/0.3, mole fraction) } \\
\hline 7.0 & Dry & 9 & $2020-12-09$ & 39.2 & 16.7 & $\mathrm{~N} / \mathrm{A}$ & 743.1 & $N / A$ & 798.3 & 759.2 & 0.006 \\
\hline 8.0 & & 5 & $2020-12-08$ & 44.8 & 19.1 & & 735.1 & & 798.3 & 759.7 & 0.071 \\
\hline 10.0 & & 4 & $2020-12-08$ & 55.9 & 23.9 & & 719.2 & & 798.3 & 759.7 & 0.072 \\
\hline 11.0 & & 6 & $2020-12-08$ & 61.5 & 26.4 & & 711.2 & & 798.4 & 759.0 & 0.171 \\
\hline 12.0 & & 1 & 2020-12-08 & 67.1 & 28.7 & & 703.1 & & 798.3 & 759.9 & 0.176 \\
\hline 13.0 & & 7 & $2020-12-08$ & 72.7 & 31.1 & & 695.4 & & 798.4 & 759.0 & 0.154 \\
\hline 14.0 & & 2 & $2020-12-08$ & 78.3 & 33.5 & & 687.1 & & 798.3 & 759.9 & 0.160 \\
\hline 15.0 & & 8 & 2020-12-09 & 83.8 & 35.9 & & 679.2 & & 798.3 & 759.8 & 0.149 \\
\hline 16.0 & & 3 & $2020-12-08$ & 89.4 & 38.2 & & 671.2 & & 798.2 & 759.5 & 0.005 \\
\hline 17.0 & & 10 & $2020-12-11$ & 95.0 & 40.7 & & 663.4 & & 798.2 & 759.6 & 0.049 \\
\hline 18.0 & & 11 & $2020-12-23$ & 100.6 & 43.1 & & 655.7 & & 798.3 & 760.2 & 0.015 \\
\hline
\end{tabular}

\section{R134a/1234yf (0.8/0.2, mole fraction)}

\begin{tabular}{|c|c|c|c|c|c|c|c|c|c|c|}
\hline Dry & 4 & $2020-12-09$ & 70.3 & 17.5 & $\mathrm{~N} / \mathrm{A}$ & 711.2 & $\mathrm{~N} / \mathrm{A}$ & 798.3 & 759.5 & 0.004 \\
\hline 12.0 & 5 & $2020-12-09$ & 76.7 & 19.1 & & 703.2 & & 798.3 & 759.6 & 0.006 \\
\hline 13.0 & 1 & $2020-12-09$ & 83.0 & 20.7 & & 695.3 & & 798.4 & 759.8 & 0.082 \\
\hline 14.0 & 2 & 2020-12-09 & 89.4 & 22.4 & & 687.3 & & 798.3 & 759.8 & 0.124 \\
\hline 15.1 & 3 & 2020-12-09 & 95.8 & 25.2 & & 678.0 & & 798.2 & 759.9 & 0.107 \\
\hline 6.0 & 6 & $2020-12-09$ & 102.2 & 25.6 & & 671.4 & & 798.5 & 760.0 & 0.034 \\
\hline 7.0 & 7 & $2020-12-10$ & 108.5 & 27.2 & & 663.0 & & 798.3 & 759.9 & 0.070 \\
\hline 8.0 & 8 & $2020-12-11$ & 115.0 & 28.7 & & 655.4 & & 798.4 & 759.9 & 0.101 \\
\hline 9.0 & 9 & $2020-12-11$ & 121.3 & 30.3 & & 647.3 & & 798.2 & 760.1 & 0.110 \\
\hline 0.0 & 10 & $2020-12-11$ & 127.8 & 31.9 & & 639.5 & & 798.3 & 759.8 & 0.033 \\
\hline
\end{tabular}

\section{R134a/1234yf (0.9/0.1, mole fraction)}

\begin{tabular}{|c|c|c|c|c|c|c|c|c|c|c|}
\hline 13.0 Dry & 4 & $2020-12-10$ & 93.4 & 10.4 & $\mathrm{~N} / \mathrm{A}$ & 695.1 & $\mathrm{~N} / \mathrm{A}$ & 798.3 & 759.8 & 0.023 \\
\hline 14.0 & 1 & $2020-12-10$ & 100.6 & 11.1 & & 687.2 & & 798.3 & 759.8 & 0.155 \\
\hline 15.0 & 2 & $2020-12-10$ & 107.8 & 12.1 & & 679.1 & & 798.3 & 760.1 & 0.070 \\
\hline 16.0 & 3 & $2020-12-10$ & 115.0 & 12.8 & & 671.2 & & 798.2 & 759.9 & 0.034 \\
\hline 17.0 & 5 & $2020-12-10$ & 122.1 & 13.5 & & 663.4 & & 798.2 & 760.0 & 0.113 \\
\hline 18.0 & 6 & $2020-12-11$ & 129.3 & 14.4 & & 655.4 & & 798.4 & 760.1 & 0.132 \\
\hline 19.0 & 7 & $2020-12-11$ & 136.6 & 15.0 & & 647.4 & & 798.4 & 759.8 & 0.016 \\
\hline
\end{tabular}

\section{R134a/1234yf (1.0/0, mole fraction)}

\begin{tabular}{llllllllllll}
13.0 & 57 & 11 & $2021-01-21$ & 103.8 & N/A & N/A & 685.2 & 10.9 & 798.3 & 759.2 & 0.023 \\
14.0 & 55 & 2 & $2021-01-15$ & 111.7 & & & 677.6 & 10.4 & 798.2 & 759.1 & 0.087 \\
15.0 & 44 & 1 & $2021-01-15$ & 119.6 & & & 671.8 & 8.3 & 798.3 & 759.5 & 0.102 \\
16.0 & 58 & 3 & $2021-01-15$ & 127.7 & & & 661.2 & 10.8 & 798.4 & 759.3 & 0.103 \\
17.0 & 60 & 4 & $2021-01-19$ & 135.7 & & & 652.8 & 11.0 & 798.2 & 759.7 & 0.030 \\
17.9 & 62 & 5 & $2021-01-19$ & 143.6 & & & 647.2 & 11.3 & 800.6 & 759.8 & 0.033 \\
19.0 & 60 & 6 & $2021-01-19$ & 151.7 & & 637.3 & 10.8 & 798.2 & 759.7 & 0.096 \\
20.0 & 61 & 7 & $2021-01-19$ & 159.7 & & & 629.3 & 10.8 & 798.4 & 759.7 & 0.095 \\
20.9 & 68 & 8 & $2021-01-19$ & 167.5 & & 620.2 & 11.9 & 798.3 & 759.6 & 0.068 \\
22.0 & 65 & 9 & $2021-01-21$ & 175.6 & & 612.9 & 11.2 & 798.3 & 759.2 & 0.053 \\
22.9 & 65 & 10 & $2021-01-21$ & 183.5 & & & 605.2 & 11.1 & 798.3 & 759.3 & 0.053 \\
\hline
\end{tabular}




\section{A.2.3. Constant Volume (2 L) Laminar Burning Velocity Measurements}

Experiment Description. The experimental approach for measuring the laminar burning velocity $\mathrm{LBV}$ in the $2-\mathrm{L}$ chamber from the pressure rise has been described in previous work 26, 27, 40,98, and this description is an updated version of that in Pagliaro et al. ${ }^{27}$. The experiment uses the same 2-L chamber and gas preparation system as described above for the modified JHPGL test. Instead of the hot-wire ignition, the LBV measurement uses an electrical spark from a capacitive discharge ignition system (based on the work of Shepherd et al. ${ }^{99}$ ). A $1 \mathrm{kV}$ to $15 \mathrm{kV}$ power supply and custom-made capacitor banks ( $1 \mathrm{nF}$ to $\left.50 \mathrm{nF}\right)$ provide variable ignition energies, with an estimated operating range of $0.05 \mathrm{~mJ}$ to $500 \mathrm{~mJ}$. Two tungsten electrodes form a gap in the center of the chamber. Thin electrodes $(0.4 \mathrm{~mm}$ diameter) minimize heat loss from the flame, and the spark gap is adjustable ( $2 \mathrm{~mm}$, typical).

Gases and mixture preparation are the same as in the JHPGL test, with the additional refrigerant HFC-152a (Honeywell). Ignition is attempted several times, while gradually increasing the capacitor charging voltage, until ignition occurs. This ensures the ignition energy is within an order of magnitude of the minimum value. The explosion pressure is recorded at $4000 \mathrm{~Hz}$ with the dynamic pressure sensor, and the product gases are immediately purged, as described above. Laminar burning velocity is determined from the pressure trace using a thermodynamic model, developed by Metghalchi and Keck ${ }^{100,101}$ and further refined by others ${ }^{102,103}$. A brief overview of the method follows; however, the current work uses an updated software package that allows for inclusion of radiation heat losses, burned gas dissociation, and accounts for extrapolation errors due to the range of selected pressure-rise data ${ }^{49}$. Nonetheless, the present data were reduced assuming adiabatic conditions and using a 2-zone model as described below.

Burning Velocity from the Pressure Trace. The content of the chamber is divided into burned and unburned zones separated by a reaction sheet, assumed to be infinitely thin, spherical, and smooth (no instabilities). Initially, the unburned gas is considered perfectly mixed and at rest. As the unburned gases react, a spatially uniform increase in pressure occurs. The burned gas is in chemical equilibrium and both the burned and unburned gases are considered as ideal, semi-perfect gases. Both zones are adiabatic, and the unburned gas is isentropically compressed as the mixture reacts in the flame sheet.

With these assumptions, the instantaneous flame radius and burning velocity can be expressed in terms of the chamber pressure by applying conservation of mass. The results are given in Eq. A.2-1 and Eq. A.2-2 (detailed formulation of the equations can be found in refs. 100,102 ),

$$
\begin{gathered}
r_{f}=R\left[1-\left(1-x_{b}\right)\left(P_{0} / P\right)^{1 / \gamma_{u}}\right]^{1 / 3} \\
S_{L}=R / 3\left(R / r_{f}\right)^{2}\left(P_{0} / P\right)^{1 / \gamma_{u}}\left(d x_{b} / d t\right)
\end{gathered}
$$

in which $r_{f}$ is the flame radius, $S_{u}$ is the laminar burning velocity, $R$ is the chamber radius, $x_{b}$ is the mass fraction of burned gas, $P$ is the instantaneous pressure, $P_{0}$ is the initial pressure, and $\gamma_{u}$ is the unburned gas specific heat ratio. To determine burning velocity, the 
mass fraction of burned gas $x_{b}$ must be related to the chamber pressure (for simplification, early studies applied a linear relationship ${ }^{104}$ ). Alternatively, a thermodynamic closed system analysis on the contents within the chamber can be performed. A two-zone approach is used in the present work. The burned gas zone also can be modeled using incremental shell volumes ${ }^{105,106}$, allowing burned gas density and temperature gradients; nonetheless, since previous studies have shown that (for adiabatic flames) inclusion of the gradients has negligible effect on the burning velocity ${ }^{101,102}$, we retain the two-zone model. The fraction of mass burned $x_{b}$ is found from simultaneous solution of the conservation of mass and energy equations given in Eq. A.2-3 and Eq. A.2-4, applied for the two zones,

$$
\begin{aligned}
& \frac{V}{M}=\int_{0}^{x_{b}} v_{b} d x+\int_{x_{b}}^{1} v_{u} d x \\
& \frac{E}{M}=\int_{0}^{x_{b}} e_{b} d x+\int_{x_{b}}^{1} e_{u} d x
\end{aligned}
$$

where $V$ is the volume of the chamber, $E$ is the total internal energy of the gases in the chamber, $M$ is the mass of the gas in the chamber, $e$ and $v$ are the internal energy and specific volume of the gas, and the subscripts $b$ and $u$ refer to the burned and unburned gas.

Thermodynamic data for the unburned and burned gases are required for model implementation. Data for all of the species are taken from the recent detailed kinetic model of Babushok et al. ${ }^{46}$.

The unburned gas properties are related to the chamber pressure through isentropic compression:

$$
T_{u}=T_{u 0}\left(\frac{P}{P_{0}}\right)^{\left(\gamma_{u}-1\right) / \gamma_{u}}
$$

in which $T_{u 0}$ is the initial unburned temperature. Since $\gamma_{u}$ is a function of the unburned gas temperature $T_{u}$ and the initial mixture composition, $T_{u}$ and $\gamma_{u}$ are solved iteratively at each pressure increment. The properties of the unburned gas mixture, $v_{u}$ and $e_{u}$, are determined from the mixture composition and temperature.

The remaining unknowns in the conservation equations $\left(v_{b}, e_{b}\right.$, and $\left.x_{b}\right)$ are found through iteration of $T_{b}\left(v_{b}\right.$ and $e_{b}$ are functions of temperature) and $x_{b}$ at each pressure increment, until the proper values of $T_{b}$ and $x_{b}$ are obtained. Burned gas species concentrations, required for the determination of $v_{b}$ and $e_{b}$, are estimated for each value of $T$ and $P$ using a constant volume equilibrium calculation performed via the open-source Cantera software package ${ }^{31}$. Once $x_{b}(P)$ is known, the burning velocity $S_{u}\left(P, T_{u}\right)$ is calculated over the experimental range of pressure and temperature using Eq. A.2-2.

Data Reduction. Figure A.2-3 shows a typical pressure trace (solid line) from an experiment $\left(\mathrm{CH}_{4}\right.$-air, $\left.\phi=1.0\right)$, along with $T_{b}, T_{u}$, and $r_{f}$, which are outputs of the two-zone model calculation. Only a portion of the pressure data is used for obtaining burning velocity, as denoted by the thick line on the pressure trace. For small $r_{f}$, the flame behavior is affected by 
flame stretch and the ignition process, and for large $r_{f}$, by heat losses to the walls and cellular instabilities; hence, typically only the central $75 \%$ of the pressure data are used. The present data reduction was performed using a new NIST-developed tool, CVDART (Constant Volume Data Reduction Tool) ${ }^{49}$ which allows for detailed examination of the effects of different data ranges. The data range selected for each test was manually selected to minimize the effects of ignition and flame instabilities due to cellular flame formation at the larger radii.

Spherically propagating flames are subject to stretch rates inversely proportional to the flame radius ${ }^{107}$,

$$
\kappa=\frac{2}{r_{f}} \frac{d r_{f}}{d t}
$$

where $\kappa$ is the stretch rate and $d r_{f} / d t$ is the flame front velocity. Figure A.2-3 also shows the stretch rate for this experiment.

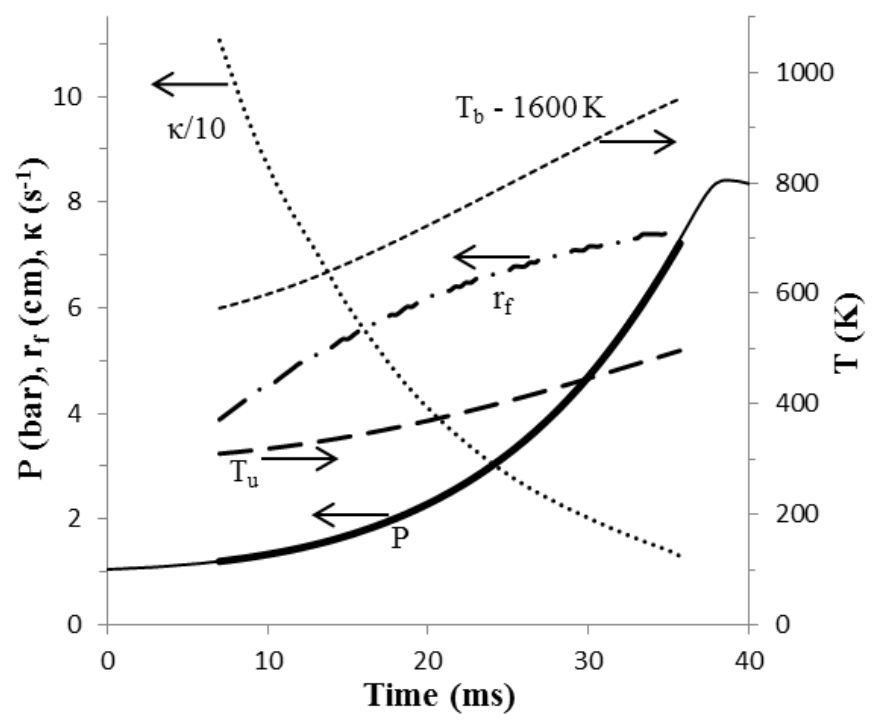

Figure A.2-3. Experimental pressure trace $P$, flame radius rf, flame stretch rate $\kappa$, and gas temperature (unburned $\mathrm{Tu}$ and burned $\mathrm{Tb}$ ) as a function of time.

A single experiment provides burning velocity data for a range of pressure and temperature of the unburned gas, and these data are fit to the equation [33]:

$$
S_{L}=S_{L, 0}\left(\frac{T}{T_{0}}\right)^{\alpha}\left(\frac{P}{P_{0}}\right)^{\beta}
$$

in which $S_{L}$ is the laminar burning velocity, $P_{0}$ is the initial pressure, $T_{0}$ is the initial temperature, $S_{L, 0}$ is the laminar burning velocity at the initial conditions; $\alpha, \beta$, and $S_{L, 0}$ are 
the fitting parameters. In the experiments performed, $T_{0}$ is $299 \pm 2 \mathrm{~K}$, and $P_{0}$ is 0.868 bar, $1 \mathrm{bar}$, and 1.13 bar, (to provide more data for the curve fit). As described in Takizawa et al. ${ }^{108}, S_{\mathrm{u}}{ }^{0}, \alpha$, and $\beta$ depend upon $\phi$ and can be fit to the following equations (2-4):

$$
\begin{gathered}
S_{L 0}=S_{L 0, \text { max }}+s_{1}\left(f-f_{\text {max }}\right)^{2}+s_{2}\left(f-f_{\text {max }}\right)^{3} \\
a=a_{1}+a_{2}(f-1) \\
b=b_{1}+b_{2}(f-1)
\end{gathered}
$$

Thus, $a_{1}, a_{2}, b_{1}, b_{2}, s_{1}, s_{2}, S_{L 0, \max }$, and $f_{\max }$ are fitting parameters to the data, with $S_{L 0, \max }$ the maximum laminar burning velocity over all $\phi$, and $\phi_{\max }$, the value of $\phi$ at which it occurs.

Figure A.2-4 shows the burning velocity of stoichiometric methane-air as a function of unburned gas temperature and chamber pressure. The light grey surface is the fit following Eq. A.2-7, and the darker grey data points (and lines through them) are the $S_{L}$ values at each combination of unburned gas pressure and temperature for a specific run, to which Eq. A.2-7 is fit.

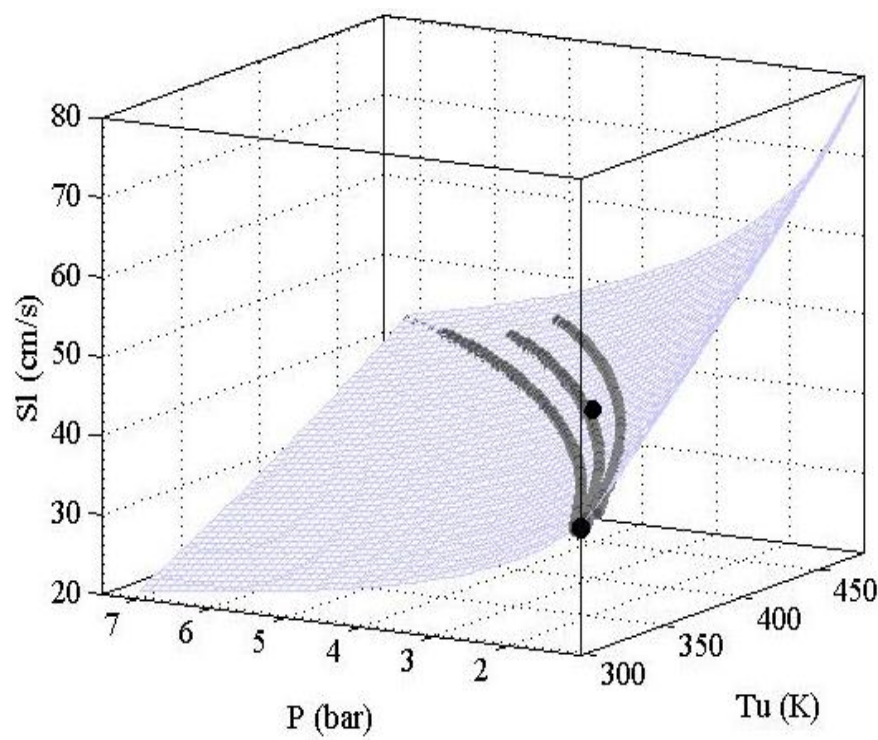

Figure A.2-4. Three-dimensional plot of burning velocity as a function of pressure $\mathrm{P}$ and unburned gas temperature Tu.

The dark grey dots represent the data from three experiments, one at each initial pressure. The light grey surface represents the fitted results using Eq. A.2-7 and the black dots are the reported $S_{L}$ at ambient $\left(T_{0}=298 \mathrm{~K}, P_{0}=1\right.$ bar) and compressed $\left(T_{0}=400 \mathrm{~K}, P_{0}=3\right.$ bar) conditions.

In the present method, spherical flame propagation is a critical condition for accurate determination of $S_{L}$ from the pressure trace. Buoyancy can distort the shape of the flame, especially for slow burning mixtures (which are of particular interest in the present work). 
Takizawa et al. ${ }^{103}$ estimated the minimum burning velocity measurable without error associated with buoyancy. Results showed that burning velocities as low as $6 \mathrm{~cm} / \mathrm{s}$ could be measured with the constant-volume method. In the present work, cellular instabilities, which also invalidate the spherical flame assumption, are monitored through inspection of the $S_{L}$ data of individual test runs. The onset of cellular instabilities is typically detected via a distinct increase in $S_{L}{ }^{105,109}$, and these data (if occurring) are omitted during the experimental data fitting.

Uncertainties. Uncertainties in the measured parameters presented as expanded uncertainties $k u_{c}$ from a combined standard uncertainty (estimated standard deviation) $u_{c}$, and a coverage factor $k=2$ (level of confidence approximately 95\%). Relative uncertainties $k u_{c} / X$ are reported, with $X$ being the measured value of the parameter under consideration. Uncertainties in initial temperature and pressure, dynamic pressure rise, agent volume fraction, equivalence ratio, and burning velocity calculation and fitting to Eq. A.2-7 are considered. The expanded relative uncertainties related to reactant mixture preparation are $1.0 \%$ for the equivalence ratio, $0.3 \%$ for the volume fraction of air, and $0.8 \%$ for the volume fraction of fuel. Uncertainty in the volume fraction of refrigerant ranged from $4 \%$ at low concentrations (volume fraction of 0.01 ) to $<1 \%$ at higher concentrations (volume fraction of 0.05). Relative uncertainties for the dynamic pressure rise, initial pressure, and initial temperature are $1.3 \%, 0.7 \%$, and $0.4 \%$. Thus, the maximum expanded relative uncertainty $\left(k u_{c} / X\right)$ for the reported experimental burning velocities is $12 \%$. This does not consider the uncertainty due to the selected data range for the extrapolation, which are discussed in detail for the present data in Kim et al. ${ }^{110}$.

Experimental Burning Velocity Test Data. Table A.2.3 lists the fitting parameters (Eq. A.2-8, to A.2.10) for the burning velocity as a function of with $\phi, T_{\mathrm{u}}$ and $P$ for pure HFC-152a and its mixtures with HFC-134a and HFO-1234yf. The corresponding raw experimental data are given in Table A.2.4. 
Table A.2-3. Fitting parameters for the variation of $S_{L}$ with $\phi, T_{\mathrm{u}}$ and $P$ for experimental burning velocity data obtained in 2-L experiment from pressure rise data for R152a/134a and R-152a/1234yf mixtures. Data presented for $298 \mathrm{~K}, 101 \mathrm{kPa}$ initial conditions, and dry air ( $<2 \%$ r.h.) of wet air $(50 \%$ r.h.) as noted.

\begin{tabular}{|c|c|c|c|c|c|c|c|c|c|}
\hline \multirow{2}{*}{$\begin{array}{l}\text { Composition } \\
\text { (mole frac) }\end{array}$} & \multirow[t]{2}{*}{ Humidity } & \multicolumn{4}{|c|}{$\mathrm{Su}_{\mathrm{u} 0}(\mathrm{~cm} / \mathrm{s})$} & \multicolumn{2}{|l|}{$\alpha$} & \multicolumn{2}{|l|}{$\beta$} \\
\hline & & Su0,max & s1 & $\phi_{\max }$ & s2 & a1 & $\mathbf{a 2}$ & b1 & b2 \\
\hline \multicolumn{10}{|l|}{ R-152a } \\
\hline$(1.0)$ & Dry & 22.92 & -85.94 & 1.10 & -13.80 & 1.25 & -0.56 & -0.06 & 0.20 \\
\hline \multicolumn{10}{|l|}{ R-152a/134a } \\
\hline$(0.8 / 0.2)$ & & 18.05 & -75.78 & 1.09 & -6.23 & 2.26 & -2.01 & -0.71 & -0.33 \\
\hline \multicolumn{10}{|l|}{$R-152 a / 134 a$} \\
\hline$(0.5 / 0.5)$ & & 9.99 & -35.48 & 1.04 & 20.44 & 1.96 & 0.21 & -0.19 & -0.17 \\
\hline \multicolumn{10}{|l|}{ R-152a/1234yf } \\
\hline$(0.5 / 0.5)$ & & 13.40 & -39.50 & 1.04 & 3.51 & 1.86 & -1.49 & -0.15 & 0.35 \\
\hline$(0.4 / 0.6)$ & & 9.38 & -50.71 & 0.99 & 61.30 & 2.48 & -3.06 & -0.21 & 0.75 \\
\hline$(0.4 / 0.6)$ & Wet & 11.61 & -13.87 & 0.88 & -11.07 & 2.36 & -2.54 & -0.27 & 0.69 \\
\hline$(0.3 / 0.7)$ & Dry & 5.74 & -44.45 & 1.00 & 77.11 & 1.12 & 2.99 & 0.19 & -0.51 \\
\hline
\end{tabular}


Table A.2-4. Experimental burning velocity data obtained in 2-L experiment from pressure rise data for R-152a/134a and R-152a/1234yf mixtures. Data presented for $298 \mathrm{~K}, 101 \mathrm{kPa}$ initial conditions, dry air (<2\% r.h.) and wet air $(50 \%$ r.h.) as noted. Blend compositions given in mole fraction.

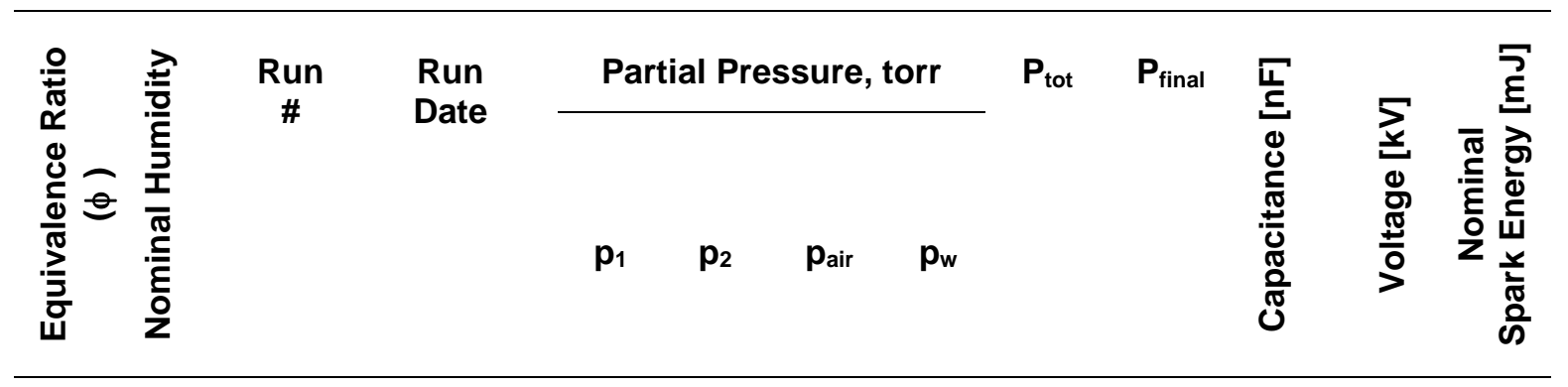

\begin{tabular}{|c|c|c|c|c|c|c|c|c|c|c|c|c|}
\hline \multicolumn{13}{|c|}{ R-152a } \\
\hline \multirow[t]{3}{*}{0.8} & Dry & 2 & 2019-12-05 & 43.6 & $\mathrm{~N} / \mathrm{A}$ & 647.9 & $\mathrm{~N} / \mathrm{A}$ & 692.2 & 659.7 & 10 & 10 & 2.5 \\
\hline & & 1 & 2019-12-05 & 50.3 & & 747.4 & & 798.3 & 760.1 & 10 & 10 & 2.5 \\
\hline & & 3 & 2019-12-09 & 57.0 & & 846.6 & & 904.3 & 860.2 & 10 & 10 & 2.5 \\
\hline \multirow[t]{3}{*}{0.9} & & 4 & $2019-12-11$ & 48.7 & & 642.9 & & 692.2 & 660.0 & 10 & 10 & 2.5 \\
\hline & & 5 & $2019-12-11$ & 56.1 & & 741.5 & & 798.3 & 760.2 & 10 & 10 & 2.5 \\
\hline & & 6 & 2019-12-11 & 63.6 & & 840.0 & & 904.3 & 860.0 & 10 & 10 & 2.5 \\
\hline \multirow[t]{3}{*}{1} & & 19 & $2019-12-16$ & 53.7 & & 637.8 & & 692.2 & 659.9 & 10 & 10 & 2.5 \\
\hline & & 20 & $2019-12-16$ & 62.0 & & 735.7 & & 798.3 & 760.2 & 10 & 10 & 2.5 \\
\hline & & 21 & $2019-12-16$ & 70.1 & & 833.5 & & 904.3 & 860.2 & 10 & 10 & 2.5 \\
\hline \multirow[t]{3}{*}{1.1} & & 7 & $2019-12-11$ & 58.6 & & 632.9 & & 692.2 & 659.9 & 10 & 10 & 2.5 \\
\hline & & 8 & $2019-12-11$ & 67.6 & & 730.1 & & 798.3 & 759.9 & 10 & 10 & 2.5 \\
\hline & & 9 & 2019-12-11 & 76.6 & & 827.0 & & 904.2 & 860.0 & 10 & 10 & 2.5 \\
\hline \multirow[t]{3}{*}{1.2} & & 10 & $2019-12-11$ & 63.5 & & 628.1 & & 692.1 & 659.7 & 10 & 10 & 2.5 \\
\hline & & 11 & $2019-12-11$ & 73.2 & & 724.5 & & 798.3 & 760.1 & 10 & 10 & 2.5 \\
\hline & & 12 & $2019-12-11$ & 82.9 & & 820.7 & & 904.3 & 859.8 & 10 & 10 & 2.5 \\
\hline \multirow[t]{3}{*}{1.3} & & 13 & $2019-12-12$ & 68.2 & & 623.3 & & 692.2 & 660.8 & 10 & 10 & 2.5 \\
\hline & & 14 & $2019-12-12$ & 78.7 & & 719.0 & & 798.4 & 760.4 & 10 & 10 & 2.5 \\
\hline & & 15 & 2019-12-12 & 89.1 & & 814.6 & & 904.3 & 860.3 & 10 & 10 & 2.5 \\
\hline \multirow[t]{3}{*}{1.4} & & 16 & $2019-12-12$ & 72.9 & & 618.7 & & 692.2 & 660.0 & 10 & 10 & 2.5 \\
\hline & & 17 & $2019-12-12$ & 84.1 & & 713.6 & & 798.3 & 760.5 & 10 & 10 & 2.5 \\
\hline & & 18 & 2019-12-12 & 95.3 & & 808.5 & & 904.3 & 859.9 & 10 & 10 & 2.5 \\
\hline \multicolumn{13}{|c|}{$R-152 a / 134 a(0.8 / 0.2)$} \\
\hline \multirow[t]{3}{*}{0.8} & Dry & 21 & 2019-01-02 & 37.8 & 9.4 & 644.4 & $\mathrm{~N} / \mathrm{A}$ & 692.2 & 659.7 & 50 & 10 & 12.5 \\
\hline & & 2 & $2019-12-20$ & 43.5 & 10.9 & 743.3 & & 798.3 & 760.5 & 50 & 10 & 12.5 \\
\hline & & 9 & 2019-12-23 & 49.3 & 12.3 & 842.0 & & 904.3 & 860.4 & 50 & 10 & 12.5 \\
\hline \multirow[t]{3}{*}{0.9} & & 20 & 2019-01-02 & 42.1 & 10.5 & 638.9 & & 692.2 & 659.7 & 50 & 10 & 12.5 \\
\hline & & 3 & $2019-12-20$ & 48.6 & 12.1 & 737.0 & & 798.3 & 760.4 & 50 & 10 & 12.5 \\
\hline & & 10 & $2019-12-23$ & 55.0 & 13.7 & 834.9 & & 904.3 & 860.1 & 50 & 10 & 12.5 \\
\hline
\end{tabular}




\begin{tabular}{ccccccccccc}
\hline 1 & 16 & $2019-01-02$ & 45.7 & 12.3 & 633.6 & 692.2 & 659.8 & 50 & 10 & 12.5 \\
& 4 & $2019-12-20$ & 53.5 & 13.4 & 730.8 & 798.3 & 760.3 & 50 & 10 & 12.5 \\
& 11 & $2019-12-23$ & 60.6 & 15.1 & 827.9 & 904.3 & 860.3 & 50 & 10 & 12.5 \\
\hline 1.1 & 1 & $2019-01-02$ & 50.6 & 12.6 & 628.3 & 692.2 & 660.1 & 50 & 10 & 12.5 \\
& 5 & $2019-12-20$ & 58.4 & 14.6 & 724.7 & 798.3 & 760.3 & 50 & 10 & 12.5 \\
& 12 & $2019-12-23$ & 66.1 & 16.5 & 821.1 & 904.3 & 860.0 & 50 & 10 & 12.5 \\
\hline 1.2 & 17 & $2019-01-02$ & 54.7 & 13.7 & 623.1 & 692.2 & 659.9 & 50 & 10 & 12.5 \\
& 6 & $2019-12-20$ & 63.1 & 15.8 & 718.8 & 798.3 & 760.1 & 50 & 10 & 12.5 \\
& 13 & $2019-12-23$ & 69.5 & 19.9 & 814.2 & 904.3 & 859.9 & 50 & 10 & 12.5 \\
\hline 1.3 & 18 & $2019-01-02$ & 58.8 & 14.7 & 618.0 & 692.2 & 659.8 & 50 & 10 & 12.5 \\
& 7 & $2019-12-20$ & 67.8 & 17.0 & 712.9 & 798.3 & 760.0 & 50 & 10 & 12.5 \\
& 14 & $2019-12-23$ & 76.8 & 19.2 & 807.6 & 904.3 & 859.8 & 50 & 10 & 12.5 \\
\hline 1.4 & 19 & $2019-01-02$ & 62.9 & 15.7 & 613.0 & 692.2 & 660.4 & 50 & 10 & 12.5 \\
& 8 & $2019-12-20$ & 72.5 & 18.1 & 707.0 & 798.3 & 760.3 & 50 & 10 & 12.5 \\
& 15 & $2019-12-23$ & 82.1 & 20.5 & 801.1 & 904.3 & 859.9 & 50 & 10 & 12.5 \\
\hline
\end{tabular}

\begin{tabular}{|c|c|c|c|c|c|c|c|c|c|c|c|c|}
\hline \multicolumn{13}{|c|}{$R-152 a / 134 a(0.5 / 0.5)$} \\
\hline \multirow[t]{4}{*}{0.8} & Dry & 12 & 2019-11-08 & 26.9 & 26.8 & 637.8 & $\mathrm{~N} / \mathrm{A}$ & 692.2 & 659.7 & 100 & 15 & 56.3 \\
\hline & & 3 & $2019-11-01$ & 31.0 & 31.0 & 736.3 & & 799.0 & 760.5 & 100 & 15 & 56.3 \\
\hline & & 4 & 2019-11-01 & 31.0 & 30.9 & 736.3 & & 798.9 & 760.8 & 100 & 15 & 56.3 \\
\hline & & 18 & $2019-11-12$ & 35.1 & 35.0 & 833.3 & & 904.1 & 859.7 & 100 & 15 & 56.3 \\
\hline \multirow[t]{3}{*}{0.85} & & 25 & $2019-11-13$ & 28.4 & 28.4 & 634.7 & & 692.1 & 659.7 & 100 & 15 & 56.3 \\
\hline & & 5 & 2019-11-01 & 32.8 & 32.7 & 732.8 & & 799.0 & 760.9 & 100 & 15 & 56.3 \\
\hline & & 24 & $2019-11-13$ & 37.1 & 37.1 & 829.2 & & 904.1 & 859.5 & 100 & 15 & 56.3 \\
\hline \multirow[t]{3}{*}{0.9} & & 11 & $2019-11-08$ & 30.0 & 29.9 & 631.7 & & 692.2 & 659.9 & 100 & 15 & 56.3 \\
\hline & & 2 & 2019-11-01 & 34.6 & 34.5 & 729.2 & & 799.0 & 760.9 & 100 & 15 & 56.3 \\
\hline & & 19 & $2019-11-12$ & 39.1 & 39.0 & 825.4 & & 904.1 & 859.7 & 100 & 15 & 56.3 \\
\hline \multirow[t]{3}{*}{1} & & 10 & 2019-11-08 & 33.0 & 32.9 & 625.7 & & 692.2 & 659.9 & 100 & 15 & 56.3 \\
\hline & & 1 & $2019-11-01$ & 38.0 & 38.0 & 722.3 & & 799.0 & 760.5 & 100 & 15 & 56.3 \\
\hline & & 17 & $2019-11-12$ & 43.0 & 42.9 & 816.8 & & 903.4 & 859.3 & 100 & 15 & 56.3 \\
\hline \multirow[t]{3}{*}{1.1} & & 13 & 2019-11-08 & 35.9 & 35.8 & 619.9 & & 692.1 & 659.7 & 100 & 15 & 56.3 \\
\hline & & 6 & 2019-11-01 & 41.4 & 41.4 & 715.4 & & 798.9 & 760.4 & 100 & 15 & 56.3 \\
\hline & & 20 & $2019-11-12$ & 46.9 & 46.8 & 809.8 & & 904.0 & 859.6 & 100 & 15 & 56.3 \\
\hline \multirow[t]{3}{*}{1.2} & & 14 & 2019-11-08 & 38.8 & 38.7 & 614.0 & & 692.2 & 659.8 & 100 & 15 & 56.3 \\
\hline & & 7 & 2019-11-01 & 44.8 & 44.7 & 708.8 & & 799.0 & 760.8 & 100 & 15 & 56.3 \\
\hline & & 23 & $2019-11-13$ & 50.7 & 50.6 & 802.2 & & 904.1 & 859.8 & 100 & 15 & 56.3 \\
\hline \multirow[t]{3}{*}{1.3} & & 15 & $2019-11-12$ & 41.6 & 41.6 & 608.4 & & 692.2 & 659.7 & 100 & 15 & 56.3 \\
\hline & & 8 & 2019-11-01 & 48.1 & 48.0 & 702.2 & & 799.0 & 760.6 & 100 & 15 & 56.3 \\
\hline & & 21 & $2019-11-13$ & 54.3 & 54.3 & 794.9 & & 904.1 & 859.7 & 100 & 15 & 56.3 \\
\hline \multirow[t]{3}{*}{1.4} & & 16 & $2019-11-12$ & 44.4 & 44.3 & 602.8 & & 692.2 & 660.3 & 100 & 15 & 56.3 \\
\hline & & 9 & 2019-11-04 & 51.2 & 51.2 & 695.8 & & 799.0 & 760.8 & 100 & 15 & 56.3 \\
\hline & & 22 & $2019-11-13$ & 58.0 & 57.9 & 787.5 & & 904.1 & 859.8 & 100 & 15 & 56.3 \\
\hline
\end{tabular}




\begin{tabular}{|c|c|c|c|c|c|c|c|c|c|c|c|c|}
\hline \multicolumn{13}{|c|}{$R-152 a / 1234 y f(0.5 / 0.5)$} \\
\hline 0.7 & Dry & 8 & $2020-02-12$ & 22.3 & 22.2 & 753.2 & $\mathrm{~N} / \mathrm{A}$ & 798.3 & 759.9 & 100 & 15 & 56.3 \\
\hline \multirow[t]{3}{*}{0.8} & Dry & 9 & $2020-02-12$ & 21.9 & 21.7 & 647.8 & & 692.1 & 659.5 & 100 & 15 & 56.3 \\
\hline & & 2 & $2020-02-11$ & 25.2 & 25.1 & 747.2 & & 798.3 & 759.7 & 100 & 15 & 56.3 \\
\hline & & 16 & $2020-02-13$ & 28.6 & 28.5 & 846.5 & & 904.2 & 859.6 & 100 & 15 & 56.3 \\
\hline \multirow[t]{3}{*}{0.9} & & 10 & $2020-02-12$ & 24.4 & 24.3 & 642.7 & & 692.1 & 659.5 & 100 & 15 & 56.3 \\
\hline & & 3 & $2020-02-12$ & 28.1 & 28.1 & 741.4 & & 798.3 & 760.1 & 100 & 15 & 56.3 \\
\hline & & 17 & $2020-02-13$ & 31.8 & 31.8 & 839.9 & & 904.2 & 859.5 & 100 & 15 & 56.3 \\
\hline \multirow[t]{3}{*}{1} & & 11 & $2020-02-13$ & 26.9 & 26.8 & 637.8 & & 692.2 & 659.5 & 100 & 15 & 56.3 \\
\hline & & 1 & $2020-02-11$ & 31.0 & 30.9 & 735.7 & & 798.3 & 759.8 & 100 & 15 & 56.3 \\
\hline & & 18 & 2020-02-14 & 35.1 & 35.0 & 833.4 & & 904.2 & 859.7 & 100 & 15 & 56.3 \\
\hline \multirow[t]{3}{*}{1.1} & & 12 & $2020-02-13$ & 29.3 & 29.3 & 632.8 & & 692.2 & 659.5 & 100 & 15 & 56.3 \\
\hline & & 4 & $2020-02-12$ & 33.8 & 33.8 & 730.0 & & 798.3 & 760.2 & 100 & 15 & 56.3 \\
\hline & & 19 & 2020-02-14 & 38.3 & 38.2 & 827.1 & & 904.3 & 860.0 & 100 & 15 & 56.3 \\
\hline \multirow[t]{3}{*}{1.2} & & 13 & $2020-02-13$ & 31.7 & 31.7 & 628.0 & & 692.1 & 660.0 & 100 & 15 & 56.3 \\
\hline & & 5 & $2020-02-12$ & 36.6 & 36.6 & 724.5 & & 798.3 & 760.1 & 100 & 15 & 56.3 \\
\hline & & 20 & 2020-02-14 & 41.5 & 41.3 & 820.7 & & 904.2 & 860.0 & 100 & 15 & 56.3 \\
\hline \multirow[t]{3}{*}{1.3} & & 14 & $2020-02-13$ & 34.1 & 34.1 & 623.2 & & 692.1 & 660.0 & 100 & 15 & 56.3 \\
\hline & & 6 & $2020-02-12$ & 39.5 & 39.3 & 718.8 & & 798.3 & 759.9 & 100 & 15 & 56.3 \\
\hline & & 21 & 2020-02-14 & 44.5 & 44.5 & 814.5 & & 904.3 & 859.8 & 100 & 15 & 56.3 \\
\hline \multirow[t]{3}{*}{1.4} & & 15 & $2020-02-13$ & 36.5 & 36.4 & 618.7 & & 692.4 & 659.7 & 100 & 15 & 56.3 \\
\hline & & 7 & $2020-02-12$ & 42.1 & 42.0 & 713.6 & & 798.3 & 759.8 & 100 & 15 & 56.3 \\
\hline & & 22 & $2020-02-14$ & 47.6 & 47.6 & 808.3 & & 904.3 & 859.7 & 100 & 15 & 56.3 \\
\hline
\end{tabular}

\begin{tabular}{|c|c|c|c|c|c|c|c|c|c|c|c|c|}
\hline \multicolumn{13}{|c|}{$R-152 a / 1234 y f(0.4 / 0.6)$} \\
\hline 0.8 & Dry & 1 & $2020-02-20$ & 17.5 & 26.1 & 647.8 & $\mathrm{~N} / \mathrm{A}$ & 692.2 & 660.0 & 100 & 15 & 56.3 \\
\hline \multirow[t]{3}{*}{0.9} & Dry & 2 & $2020-02-20$ & 19.5 & 29.2 & 647.8 & & 692.2 & 659.9 & 100 & 15 & 56.3 \\
\hline & & 8 & $2020-02-21$ & 22.5 & 33.7 & 642.8 & & 798.3 & 760.4 & 100 & 15 & 56.3 \\
\hline & & 14 & $2020-02-21$ & 25.5 & 38.2 & 741.4 & & 904.3 & 860.1 & 100 & 15 & 56.3 \\
\hline \multirow[t]{3}{*}{1} & & 3 & $2020-02-20$ & 21.5 & 32.2 & 839.9 & & 692.2 & 659.9 & 100 & 15 & 56.3 \\
\hline & & 9 & $2020-02-21$ & 24.8 & 37.1 & 637.8 & & 798.3 & 760.0 & 100 & 15 & 56.3 \\
\hline & & 15 & 2020-02-24 & 28.1 & 42.0 & 735.6 & & 904.3 & 860.0 & 100 & 15 & 56.3 \\
\hline \multirow[t]{3}{*}{1.1} & & 4 & $2020-02-20$ & 23.5 & 35.1 & 833.4 & & 692.2 & 659.8 & 100 & 15 & 56.3 \\
\hline & & 10 & $202 C$ & 27.1 & 40.5 & 633.0 & & 798.3 & 759.9 & 100 & 15 & 56.3 \\
\hline & & 16 & 2020-02-24 & 30.7 & 45.9 & 730.0 & & 904.3 & 859.8 & 100 & 15 & 56.3 \\
\hline \multirow[t]{3}{*}{1.2} & & 5 & $2020-02-20$ & 25.4 & 38.0 & 826.9 & & 692.2 & 659.7 & 100 & 15 & 56.3 \\
\hline & & 11 & $2020-02-21$ & 29.3 & 43.8 & 628.1 & & 798.3 & 760.2 & 100 & 15 & 56.3 \\
\hline & & 17 & 2020-02-24 & 33.2 & 49.7 & 724.4 & & 904.3 & 859.8 & 100 & 15 & 56.3 \\
\hline \multirow[t]{3}{*}{1.3} & & 6 & $2020-02-20$ & 27.3 & 40.9 & 820.6 & & 692.2 & 659.7 & 100 & 15 & 56.3 \\
\hline & & 12 & $2020-02-21$ & 31.5 & 47.1 & 623.2 & & 798.2 & 760.1 & 100 & 15 & 56.3 \\
\hline & & 18 & 2020-02-24 & 35.7 & 53.4 & 718.8 & & 904.3 & 859.8 & 100 & 15 & 56.3 \\
\hline \multirow[t]{2}{*}{1.4} & & 7 & $2020-02-20$ & 29.2 & 43.7 & 814.4 & & 692.1 & 659.6 & 100 & 15 & 56.3 \\
\hline & & 13 & $2020-02-21$ & 33.7 & 50.4 & 618.6 & & 798.2 & 760.0 & 100 & 15 & 56.3 \\
\hline
\end{tabular}




\begin{tabular}{|c|c|c|c|c|c|c|c|c|c|c|c|c|}
\hline & & 19 & 2020-02-24 & 38.1 & 57.1 & 713.4 & & 904.3 & 859.7 & 100 & 15 & 56.3 \\
\hline \multirow[t]{3}{*}{0.9} & 46.1 & 16 & Feb-9-2020 & 19.2 & 28.8 & 636.2 & 8.2 & 692.2 & 659.8 & 100 & 15 & 56.3 \\
\hline & 41.7 & 18 & Feb-9-2020 & 22.1 & 33.3 & 734.7 & 8.6 & 798.4 & 759.8 & 100 & 15 & 56.3 \\
\hline & 39.0 & 17 & Feb-9-2020 & 25.0 & 37.7 & 832.7 & 9.1 & 904.3 & 859.8 & 100 & 15 & 56.3 \\
\hline \multirow[t]{3}{*}{1} & 47.6 & 3 & Feb-1-2020 & 21.2 & 31.9 & 630.6 & 8.4 & 692.1 & 659.7 & 100 & 15 & 56.3 \\
\hline & 45.0 & 1 & Feb-1-2020 & 24.3 & 36.7 & 728.0 & 9.2 & 798.3 & 759.5 & 100 & 15 & 56.3 \\
\hline & 40.2 & 2 & Feb-1-2020 & 27.7 & 41.4 & 825.6 & 9.3 & 904.2 & 859.5 & 100 & 15 & 56.3 \\
\hline \multirow[t]{3}{*}{1.1} & 51.8 & 6 & Feb-2-2020 & 23.2 & 34.7 & 625.1 & 9.1 & 692.1 & 659.1 & 100 & 15 & 56.3 \\
\hline & 44.6 & 4 & Feb-2-2021 & 26.7 & 40.1 & 722.3 & 9.1 & 798.4 & 759.1 & 100 & 15 & 56.3 \\
\hline & 39.6 & 5 & Feb-2-2022 & 30.3 & 45.3 & 819.4 & 9.1 & 904.2 & 859.7 & 100 & 15 & 56.3 \\
\hline \multirow[t]{3}{*}{1.2} & 49.8 & 7 & Feb-2-2020 & 24.9 & 37.5 & 620.9 & 8.7 & 692.2 & 659.9 & 100 & 15 & 56.3 \\
\hline & 42.2 & 9 & Feb-3-2020 & 28.8 & 43.3 & 716.9 & 9.0 & 798.3 & 759.9 & 100 & 15 & 56.3 \\
\hline & 38.3 & 8 & Feb-3-2020 & 32.7 & 49.0 & 812.8 & 9.6 & 904.3 & 859.7 & 100 & 15 & 56.3 \\
\hline \multirow[t]{3}{*}{1.3} & 49.2 & 11 & Feb-4-2020 & 26.9 & 40.4 & 616.2 & 8.5 & 692.1 & 659.4 & 100 & 15 & 56.3 \\
\hline & 44.6 & 10 & Feb-4-2020 & 31.2 & 46.4 & 711.6 & 8.9 & 798.3 & 758.9 & 100 & 15 & 56.3 \\
\hline & 41.8 & 12 & Feb-4-2020 & 35.2 & 52.7 & 806.8 & 9.5 & 904.3 & 859.4 & 100 & 15 & 56.3 \\
\hline \multirow[t]{3}{*}{1.4} & 50.2 & 14 & Feb-4-2020 & 28.8 & 43.2 & 611.5 & 8.6 & 692.1 & 659.3 & 100 & 15 & 56.3 \\
\hline & 45.2 & 13 & Feb-4-2020 & 33.2 & 49.8 & 706.1 & 9.0 & 798.3 & 759.3 & 100 & 15 & 56.3 \\
\hline & 41.3 & 15 & Feb-5-2020 & 37.5 & 56.5 & 800.9 & 9.3 & 904.3 & 859.7 & 100 & 15 & 56.3 \\
\hline
\end{tabular}

\section{R-152a/R234yf (0.3/0.7)}

\begin{tabular}{|c|c|c|c|c|c|c|c|c|c|c|c|c|}
\hline 0.9 & Dry & 1 & 2020-02-17 & 16.9 & 39.2 & 742.7 & $\mathrm{~N} / \mathrm{A}$ & 798.4 & 760.8 & 100 & 15 & 56.3 \\
\hline \multirow[t]{3}{*}{1} & & 7 & 2020-02-17 & 16.2 & 37.5 & 639.1 & & 692.1 & 659.8 & 100 & 15 & 56.3 \\
\hline & & 2 & 2020-02-17 & 18.6 & 43.3 & 737.0 & & 798.4 & 760.6 & 100 & 15 & 56.3 \\
\hline & & 12 & 2020-02-19 & 21.2 & 48.9 & 834.8 & & 904.2 & 859.9 & 100 & 15 & 56.3 \\
\hline \multirow[t]{3}{*}{1.1} & & 8 & 2020-02-17 & 17.7 & 40.9 & 634.2 & & 692.2 & 659.9 & 100 & 15 & 56.3 \\
\hline & & 3 & $2020-02-17$ & 20.4 & 47.2 & 731.4 & & 798.4 & 760.5 & 100 & 15 & 56.3 \\
\hline & & 13 & 2020-02-19 & 23.0 & 53.6 & 828.2 & & 904.2 & 859.9 & 100 & 15 & 56.3 \\
\hline \multirow[t]{3}{*}{1.2} & & 9 & 2020-02-19 & 19.0 & 44.4 & 629.2 & & 692.2 & 660.0 & 100 & 15 & 56.3 \\
\hline & & 4 & 2020-02-17 & 22.1 & 51.0 & 725.7 & & 798.2 & 760.2 & 100 & 15 & 56.3 \\
\hline & & 14 & 2020-02-19 & 25.0 & 57.8 & 822.0 & & 904.2 & 859.9 & 100 & 15 & 56.3 \\
\hline \multirow[t]{3}{*}{1.3} & & 10 & 2020-02-19 & 20.5 & 47.8 & 624.6 & & 692.3 & 660.0 & 100 & 15 & 56.3 \\
\hline & & 5 & $2020-02-17$ & 23.7 & 55.0 & 720.3 & & 798.3 & 760.2 & 100 & 15 & 56.3 \\
\hline & & 15 & 2020-02-19 & 26.8 & 62.3 & 815.8 & & 904.2 & 859.7 & 100 & 15 & 56.3 \\
\hline \multirow[t]{3}{*}{1.4} & & 11 & 2020-02-19 & 22.0 & 51.0 & 619.9 & & 692.3 & 660.6 & 100 & 15 & 56.3 \\
\hline & & 6 & $2020-02-17$ & 25.3 & 58.9 & 714.8 & & 798.4 & 759.9 & 100 & 15 & 56.3 \\
\hline & & 16 & 2020-02-19 & 29.5 & 65.7 & 809.7 & & 904.2 & 859.6 & 100 & 15 & 56.3 \\
\hline
\end{tabular}




\section{A.3 Task 4 - Tabulated Flow-Boiling Heat-Transfer Measurements}

Table A.3-1. Measured flow-boiling heat-transfer data for R-515B, R-450A, R-513A, and HFC-134a.

Listed are Nusselt number $\mathrm{Nu}$, thermodynamic quality $x_{\mathrm{q}}$, reduced pressure $p$, Boiling number Bo, Bond number $\mathrm{B}_{\text {nd }}$, Reynolds number Re, and the relative combined, expanded ( $k$ $=2$ ) uncertainty in the Nusselt number $U_{\text {c. }}$ Data are presented in the sequence measured. The last column provides the test refrigerant.

\begin{tabular}{|c|c|c|c|c|c|c|c|}
\hline 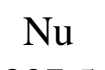 & & $D_{r}$ & 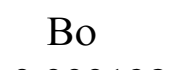 & nd & . & 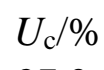 & \\
\hline 7.5 & 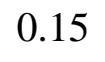 & & 0128 & & & 27.0 & \\
\hline & 29 & & & & & & \\
\hline & 40 & & & & & & \\
\hline & 61 & & & & & & \\
\hline & & & & & & & \\
\hline & 99 & & & & & & \\
\hline 6.7 & 99 & & & & & & \\
\hline & & & & & & & \\
\hline & & & & & & & \\
\hline & & & & & & & \\
\hline & & & & & & & \\
\hline & & & & & & & \\
\hline & & & & & & & \\
\hline & & & & & & & \\
\hline & & & & & & .0 & \\
\hline & & & & & & & \\
\hline & 26 & & & & & & \\
\hline & & & & & & & \\
\hline & & & & & & & \\
\hline & & & & & & .0 & \\
\hline & & & & & & .0 & \\
\hline & & & & & & & \\
\hline & & & & & & & \\
\hline & & & & & & & \\
\hline & & & & & & & \\
\hline & & & & & & .0 & \\
\hline & & & & & & .0 & \\
\hline & & & & & & .0 & \\
\hline & & & & & & 3.0 & \\
\hline & & & & & & & \\
\hline & & & & & & .0 & \\
\hline & & & & & & 23.0 & \\
\hline 35.4 & 0.59 & 0.10 & 0.000390 & 0.0225 & 3848.5 & 24.0 & \\
\hline
\end{tabular}


Table A.3-1. (continued)

\begin{tabular}{|c|c|c|c|c|c|c|c|}
\hline $\mathrm{Nu}$ & $x_{\mathrm{q}}$ & $P_{\mathrm{r}}$ & Bo & $B_{\mathrm{nd}}$ & $\mathrm{Re}$ & $U_{\mathrm{c}} / \%$ & fluid \\
\hline .1 & 76 & 10 & 00432 & 0223 & 0.6 & 26.0 & \\
\hline 1.8 & 0.97 & 0.10 & 0.000478 & 0.0223 & 3829.8 & 29.0 & R-513A \\
\hline 0.0 & 0.24 & 0.10 & 0.000293 & 0.0223 & 3969.0 & 25.0 & R-513A \\
\hline 337.8 & 0.32 & 0.10 & 0.000320 & 0.0223 & 3967.4 & 24.0 & R-513A \\
\hline 330.9 & 0.46 & 0.10 & 0.000366 & 0.0223 & 3963.0 & 23.0 & R-513A \\
\hline 323.1 & 0.62 & 0.10 & 0.000408 & 0.0223 & 3957.0 & 23.0 & R-513A \\
\hline 313.6 & 0.79 & 0.10 & 0.000452 & 0.0 & 3948.7 & 24.0 & \\
\hline 302.3 & 0.99 & 0.10 & 0.000499 & 0.0221 & 3937.4 & 26.0 & R-513A \\
\hline 309.4 & 0.21 & 0.10 & 0.000252 & 0.0223 & 4170.2 & 25.0 & \\
\hline 306.9 & 0.27 & 0.10 & 0.000275 & 0.0223 & 4168.7 & 24.0 & $\mathrm{R}-513 \mathrm{~A}$ \\
\hline 300.2 & 0.40 & 0.10 & 0.0 & & 4.2 & 23.0 & \\
\hline 292.1 & 0.53 & 0.10 & 0.000351 & 0.0223 & 4158.1 & 23.0 & $3 \mathrm{~A}$ \\
\hline 282.7 & 0.68 & 0.10 & 0.000 & 0.0223 & 4149.9 & 24.0 & \\
\hline 272.2 & 0.87 & 0.10 & 0.000429 & 0.0221 & 4138.4 & 26.0 & $\mathrm{R}-513 \mathrm{~A}$ \\
\hline 201.8 & 0.05 & 0.11 & 0.000 & 0.0 & 5732.2 & 22.0 & R- \\
\hline 201.3 & 0.13 & 0.11 & 0.0 & 0.0 & 5728.1 & 21.0 & \\
\hline 198.2 & 0.19 & 0.10 & & & & 20.0 & \\
\hline 191.8 & 0.24 & 0.10 & 0.000111 & 0.0225 & 5718.7 & 21.0 & $\mathrm{R}-513 \mathrm{~A}$ \\
\hline 177.4 & 0.28 & 0.10 & 0.000083 & 0.0225 & 5712.3 & 29.0 & R-513A \\
\hline 208.2 & 0.20 & 0.10 & 200 & 0.0223 & 2673.0 & 29.0 & $\mathrm{R}-$ \\
\hline 207.4 & 0.25 & 0.10 & 0.0 & & 2672.3 & 27.0 & \\
\hline 204.8 & 0.35 & 0.10 & 0.000249 & 0.0223 & 2670.5 & 25.0 & R-513A \\
\hline 201.6 & 0.45 & 0.10 & 0.000278 & 0.0223 & 2668.2 & 25.0 & R-513A \\
\hline 197.6 & 0.58 & 0.10 & & 0.0223 & & 25.0 & $\mathrm{R}-513 \mathrm{~A}$ \\
\hline 192.8 & 0.72 & 0.10 & 0.000341 & 0.0221 & 2661.0 & 27.0 & R-513A \\
\hline 168.4 & 0.04 & 0.10 & 0.000211 & 0.0225 & 6109.5 & 18.0 & $\mathrm{R}-513 \mathrm{~A}$ \\
\hline 172.4 & 0.13 & 0.10 & 0.000180 & 0.0222 & 6104.1 & 17.0 & $\mathrm{R}-513 \mathrm{~A}$ \\
\hline 174.9 & 0.20 & 0.10 & 0.000151 & 0.0222 & 6097.9 & 16.0 & $R-513 A$ \\
\hline 174.7 & 0.25 & 0.10 & & & & 17.0 & $\mathrm{R}-513 \mathrm{~A}$ \\
\hline 169.4 & 0.30 & 0.10 & 0.000093 & 0.0223 & 6082.3 & 24.0 & $R-513 A$ \\
\hline 183.7 & 0.28 & 0.10 & 0.000194 & 0.0222 & 2675.8 & 30.0 & $\mathrm{R}-513 \mathrm{~A}$ \\
\hline 184.0 & 0.28 & 0.10 & 0.000194 & 0.0222 & 2675.1 & 30.0 & $\mathrm{R}-513 \mathrm{~A}$ \\
\hline 182.4 & 0.28 & 0.10 & 0.000196 & 0.0223 & 2647.4 & 30.0 & $\mathrm{R}-513 \mathrm{~A}$ \\
\hline 219.1 & 0.33 & 0.10 & 0.000231 & 0.0223 & 2653.5 & 30.0 & R-513A \\
\hline 205.2 & 0.31 & 0.10 & 0.000217 & 0.0223 & 2656.5 & 30.0 & $R-513 A$ \\
\hline 148.3 & 0.08 & 0.11 & 0.000077 & 0.0225 & 6063.3 & 30.0 & $R-513 A$ \\
\hline 145.1 & 0.10 & 0.11 & 0.000086 & 0.0225 & 6062.2 & 26.0 & $\mathrm{R}-513 \mathrm{~A}$ \\
\hline 315.8 & 0.18 & 0.10 & 0.000248 & 0.0225 & 6046.8 & 20.0 & R-513A \\
\hline 309.9 & 0.25 & 0.10 & 0.000271 & 0.0225 & 6043.5 & 19.0 & R-513A \\
\hline
\end{tabular}


Table A.3-1. (continued)

\begin{tabular}{|c|c|c|c|c|c|c|c|}
\hline $\mathrm{Nu}$ & $x_{\mathrm{q}}$ & $P_{\mathrm{r}}$ & Bo & $B_{\text {nd }}$ & $\mathrm{Re}$ & $U_{\mathrm{c}} / \%$ & fluid \\
\hline 3 & 37 & 10 & 00309 & 222 & 6033.5 & 18.0 & 1 \\
\hline 285.9 & 0.50 & 0.10 & 0.000345 & 0.0223 & 6019.1 & 18.0 & R-513A \\
\hline 272.7 & 0.66 & 0.10 & .000382 & 0.0223 & 5998.8 & 19.0 & $2-513 A$ \\
\hline 258.1 & 0.84 & 0.10 & 0.000422 & 0.0221 & 5970.2 & 20.0 & R-513A \\
\hline 162.7 & 0.04 & 0.11 & 0.000160 & 0.0224 & 7327.9 & 19.0 & R-513A \\
\hline 164.6 & 0.10 & 0.11 & 0.000135 & 0.0225 & 7320.8 & 18.0 & $\mathrm{R}-513 \mathrm{~A}$ \\
\hline 164.2 & 0.16 & 0.11 & 0.000113 & 0.0225 & 7312.8 & 17.0 & \\
\hline 161.1 & 0.20 & 0.10 & 0.000092 & 0.0225 & 7304.0 & 18.0 & R-513A \\
\hline 151.2 & 0.23 & 0.10 & 0.000068 & 0.0225 & 7292.5 & 25.0 & \\
\hline 204.6 & 0.07 & 0.11 & 0.000248 & 0.0224 & 4385.6 & 23.0 & R-513A \\
\hline 02.8 & 0.17 & 0.11 & 0.0 & 0.0 & & 22.0 & \\
\hline 198.5 & 0.25 & 0.11 & 0.000175 & 0.0225 & 4379.7 & 21.0 & $3 \mathrm{~A}$ \\
\hline 190.6 & 0.31 & 0.11 & 0.0 & 0.0 & 4376.5 & 22.0 & \\
\hline 174.7 & 0.37 & 0.11 & 0.000105 & 0.0225 & 4372.4 & 30.0 & R-513A \\
\hline 35.9 & 0.08 & 0.11 & 0.00 & 0.0 & 430 & 23.0 & $3 \mathrm{~A}$ \\
\hline 34.9 & 0.20 & 0.11 & 0.0 & 0.0 & 4305.5 & 22.0 & \\
\hline 231.5 & 0.29 & 0.11 & 3 & & 43 & 21.0 & \\
\hline 224.7 & 0.36 & 0.10 & 0.000167 & 0.0225 & 4299.3 & 22.0 & R-513A \\
\hline 209.4 & 0.43 & 0.10 & 0.000125 & 0.0225 & 4295.3 & 30.0 & R-513A \\
\hline 250.7 & 0.12 & 0.11 & & & & 18.0 & \\
\hline 251.4 & 0.31 & 0.11 & 0.000389 & 0.0225 & 4242.7 & 17.0 & R-513A \\
\hline 249.3 & 0.46 & 0.10 & 0.000326 & 0.0225 & 4237.1 & 16.0 & R-513A \\
\hline 244.3 & 0.58 & 0.10 & 0.000268 & 0.0225 & 4231.2 & 17.0 & R-513A \\
\hline 231.5 & 0.68 & 0.10 & & & & 23.0 & $513 \mathrm{~A}$ \\
\hline 194.6 & 0.05 & 0.10 & 0.000232 & 0.0 & 7621.9 & 16.0 & R-513A \\
\hline 199.1 & 0.15 & 0.10 & 0.000197 & 0.0225 & 7611.6 & 15.0 & $\mathrm{R}-513 \mathrm{~A}$ \\
\hline 201.1 & 0.23 & 0.10 & 0.000165 & 0.0222 & 7599.5 & 14.0 & R-513A \\
\hline 199.9 & 0.29 & 0.10 & & & 7586.0 & 15.0 & R-513A \\
\hline 192.2 & 0.34 & 0.10 & 0.000100 & & 7567.9 & 21.0 & R-513A \\
\hline 230.7 & 0.13 & 0.11 & 0.000496 & 0.0224 & 2301.6 & 26.0 & R-513A \\
\hline 230.6 & 0.34 & 0.11 & 0.000426 & 0.0225 & 2300.0 & 24.0 & R-513A \\
\hline 228.8 & 0.51 & 0.11 & 0.000362 & 0.0225 & 2298.5 & 23.0 & R-513A \\
\hline 225.3 & 0.63 & 0.11 & 0.000303 & 0.0225 & 2297.1 & 25.0 & R-513A \\
\hline 219.5 & 0.08 & 0.11 & 0.000327 & 0.0225 & 7652.6 & 16.0 & R-513A \\
\hline 225.9 & 0.22 & 0.10 & 0.000279 & 0.0225 & 7637.3 & 14.0 & R-513A \\
\hline 230.7 & 0.33 & 0.10 & 0.000235 & 0.0222 & 7619.0 & 13.0 & R-513A \\
\hline 232.3 & 0.41 & 0.10 & 0.000194 & 0.0223 & 7598.4 & 14.0 & $\mathrm{R}-513 \mathrm{~A}$ \\
\hline 227.9 & 0.49 & 0.10 & 0.000147 & 0.0223 & 7570.3 & 19.0 & R-513A \\
\hline 220.8 & 0.52 & 0.10 & 0.000121 & 0.0221 & 7552.8 & 28.0 & R-513A \\
\hline
\end{tabular}


Table A.3-1. (continued)

\begin{tabular}{|c|c|c|c|c|c|c|c|}
\hline $\mathrm{Nu}$ & $x_{\mathrm{q}}$ & $P_{\mathrm{r}}$ & 30 & $B_{\mathrm{nd}}$ & $\mathrm{Re}$ & $U_{\mathrm{c}} / \%$ & fluid \\
\hline .1 & 0.58 & 10 & 0108 & 218 & 0 & 3.0 & \\
\hline 9.8 & 0.61 & 0.09 & 0.000108 & 0.0216 & 7363.3 & 30.0 & R-513A \\
\hline 6.0 & 0.08 & 0.08 & .000084 & 0197 & 4810.7 & 28.0 & $\mathrm{R}-450 \mathrm{~A}$ \\
\hline 135.8 & 0.11 & 0.08 & 0.000094 & 0.0197 & 4810.5 & 24.0 & $\mathrm{R}-450 \mathrm{~A}$ \\
\hline 261.5 & 0.19 & 0.08 & 0.000235 & 0.0195 & 4798.4 & 19.0 & \\
\hline 59.7 & 0.25 & 0.08 & 0.000257 & 0.0195 & 4797.9 & 18.0 & R-450A \\
\hline & 0.37 & 0.08 & 00294 & 0195 & 4793.3 & 18.0 & \\
\hline 245.1 & 0.49 & 0.08 & 0.000328 & 0.0195 & 4785.0 & 18.0 & $\mathrm{R}-450 \mathrm{~A}$ \\
\hline 235.2 & 0.64 & 0.08 & 0.000363 & 0.0193 & 4772.1 & 19.0 & \\
\hline 223.8 & 0.81 & 0.08 & 0.000402 & 0.0194 & 4752.6 & 21.0 & $\mathrm{R}-450 \mathrm{~A}$ \\
\hline 129.3 & 0.06 & 0.08 & & 95 & & 28.0 & \\
\hline 127.2 & 0.07 & 0.08 & 0.000057 & 0.0195 & 7085.8 & 24.0 & $\mathrm{R}-4$ \\
\hline 264.0 & 0.13 & 0.08 & 52 & 96 & 7056.5 & 18.0 & \\
\hline 259.4 & 0.17 & 0.08 & 0.000177 & 0.0196 & 7054.0 & 17.0 & $\mathrm{R}-450 \mathrm{~A}$ \\
\hline 247.2 & 0.25 & 0.08 & 0.000202 & 94 & 7040.9 & 16.0 & $\mathrm{R}-4$ \\
\hline 233.0 & 0.34 & 0.08 & 0.000 & 94 & 7018.5 & 16.0 & \\
\hline 217.5 & 0.44 & 0.08 & & & & 17.0 & \\
\hline 200.9 & 0.56 & 0.07 & 0.000276 & 0.0191 & 6934.2 & 17.0 & $\mathrm{R}-450 \mathrm{~A}$ \\
\hline 139.3 & 0.11 & 0.08 & 0.000098 & 0.0195 & 3818.6 & 28.0 & $\mathrm{R}-4$ \\
\hline 253.9 & 0.19 & 0.08 & 227 & 0.0195 & 1.8 & 23.0 & $\mathrm{R}-\mathrm{C}$ \\
\hline 255.1 & 0.25 & 0.08 & & & & 23.0 & \\
\hline 253.0 & 0.37 & 0.08 & 0.000283 & 0.0195 & 3810.3 & 23.0 & $\mathrm{R}-450 \mathrm{~A}$ \\
\hline 248.4 & 0.49 & 0.08 & 0.000 & 0.0 & 3806.2 & 24.0 & $\mathrm{R}-450 \mathrm{~A}$ \\
\hline 241.5 & 0.62 & 0.08 & & & & 26.0 & $\mathrm{R}-450 \mathrm{~A}$ \\
\hline 232.6 & 0.79 & 0.08 & 0.000388 & & 3788.9 & 28.0 & $\mathrm{R}-450 \mathrm{~A}$ \\
\hline 191.7 & 0.21 & 0.08 & 0.000209 & 0.0195 & 2137.3 & 29.0 & $\mathrm{R}-450 \mathrm{~A}$ \\
\hline 196.4 & 0.27 & 0.08 & 0.000228 & 0.0195 & 2137.6 & 28.0 & $\mathrm{R}-450 \mathrm{~A}$ \\
\hline 202.4 & 0.37 & 0.08 & 0.000261 & 0.0195 & 2138.1 & 29.0 & $\mathrm{R}-450 \mathrm{~A}$ \\
\hline 209.1 & 0.78 & 0.08 & 0.000382 & 0.0192 & 4845.5 & 19.0 & $\mathrm{R}-450 \mathrm{~A}$ \\
\hline 123.1 & 0.06 & 0.08 & 0.000049 & 0.0195 & 7014.5 & 29.0 & R-450A \\
\hline 120.9 & 0.07 & 0.08 & 0.000055 & 0.0195 & 7012.9 & 25.0 & $\mathrm{R}-450 \mathrm{~A}$ \\
\hline 245.5 & 0.12 & 0.08 & 0.000153 & 0.0196 & 6982.2 & 19.0 & $\mathrm{R}-450 \mathrm{~A}$ \\
\hline 241.0 & 0.16 & 0.08 & 0.000167 & 0.0196 & 6979.1 & 18.0 & $\mathrm{R}-450 \mathrm{~A}$ \\
\hline 229.6 & 0.24 & 0.08 & 0.000191 & 0.0194 & 6965.6 & 17.0 & $\mathrm{R}-450 \mathrm{~A}$ \\
\hline 216.7 & 0.32 & 0.08 & 0.000213 & 0.0194 & 6943.3 & 17.0 & $\mathrm{R}-450 \mathrm{~A}$ \\
\hline 202.8 & 0.42 & 0.08 & 0.000235 & 0.0192 & 6910.3 & 17.0 & $\mathrm{R}-450 \mathrm{~A}$ \\
\hline 187.8 & 0.53 & 0.07 & 0.000260 & 0.0191 & 6861.9 & 18.0 & $\mathrm{R}-450 \mathrm{~A}$ \\
\hline 132.8 & 0.11 & 0.08 & 0.000097 & 0.0195 & 3856.4 & 27.0 & $\mathrm{R}-450 \mathrm{~A}$ \\
\hline 239.5 & 0.19 & 0.08 & 0.000226 & 0.0195 & 3849.3 & 21.0 & $\mathrm{R}-450 \mathrm{~A}$ \\
\hline
\end{tabular}


Table A.3-1. (continued)

\begin{tabular}{|c|c|c|c|c|c|c|c|}
\hline $\mathrm{Nu}$ & $x_{\mathrm{q}}$ & $P_{\mathrm{r}}$ & Bo & $B_{\mathrm{nd}}$ & $\mathrm{Re}$ & $U_{\mathrm{c}} / \%$ & fluid \\
\hline 3.6 & 25 & 08 & .000247 & 0.0195 & 3849.4 & 20.0 & $\mathrm{R}-450 \mathrm{~A}$ \\
\hline 34.8 & 0.36 & 0.08 & 0.000282 & 0.0195 & 3847.6 & 20.0 & $\mathrm{R}-450 \mathrm{~A}$ \\
\hline 229.2 & 0.48 & 0.08 & 0.000315 & 0.0195 & 3843.7 & 20.0 & $\mathrm{R}-450 \mathrm{~A}$ \\
\hline 222.4 & 0.62 & 0.08 & 0.000350 & 0.0194 & 3837.2 & 21.0 & $\mathrm{R}-450 \mathrm{~A}$ \\
\hline 214.3 & 0.79 & 0.08 & 0.000387 & 0.0194 & 3827.4 & 23.0 & $\mathrm{R}-450 \mathrm{~A}$ \\
\hline 134.3 & 0.09 & 0.08 & 0.000089 & 0.0195 & 3861.3 & 30.0 & $\mathrm{R}-450 \mathrm{~A}$ \\
\hline 133.9 & 0.11 & 0.08 & 0.000100 & 0.0195 & 3861.6 & 27.0 & $\mathrm{R}-450 \mathrm{~A}$ \\
\hline 246.5 & 0.20 & 0.08 & 0.000236 & 0.0195 & 3855.9 & 21.0 & $\mathrm{R}-450 \mathrm{~A}$ \\
\hline 45.5 & 0.26 & 0.08 & 0.000257 & 0.0195 & 3856.2 & 20.0 & $\mathrm{R}-450 \mathrm{~A}$ \\
\hline 1.6 & 0.38 & 0.08 & 0.000 & & & 19.0 & $50 \mathrm{~A}$ \\
\hline 235.6 & 0.50 & 0.08 & 0.000329 & 0.0195 & 3851.1 & 20.0 & $\mathrm{R}-450 \mathrm{~A}$ \\
\hline 228.5 & 0.65 & 0.08 & 0.000364 & 0.0195 & 3844.8 & 21.0 & $\mathrm{R}-450 \mathrm{~A}$ \\
\hline 19.8 & 0.82 & 0.08 & 0.000404 & 0.0193 & 3834.8 & 23.0 & $\mathrm{R}-450 \mathrm{~A}$ \\
\hline 130.7 & 0.09 & 0.08 & 0.000089 & & 3969.6 & 29.0 & $\mathrm{R}-450 \mathrm{~A}$ \\
\hline 130.6 & 0.11 & 0.08 & 0.000100 & 0.0195 & & 26.0 & $\mathrm{R}-450 \mathrm{~A}$ \\
\hline 242.4 & 0.20 & 0.08 & 0.000240 & 0.0195 & 3961.2 & 20.0 & $\mathrm{R}-450 \mathrm{~A}$ \\
\hline 241.4 & 0.26 & 0.08 & 0.000262 & 0.0195 & 3961.1 & 19.0 & $\mathrm{R}-450 \mathrm{~A}$ \\
\hline 237.0 & 0.38 & 0.08 & 0.000299 & 0.0 & 3959.0 & 19.0 & $\mathrm{R}-450 \mathrm{~A}$ \\
\hline 231.4 & 0.51 & 0.08 & 0.000335 & & & 19.0 & $\mathrm{R}-450 \mathrm{~A}$ \\
\hline 224.3 & 0.66 & 0.08 & 0.000371 & 0.0194 & 3947.7 & 21.0 & $\mathrm{R}-450 \mathrm{~A}$ \\
\hline 216.0 & 0.83 & 0.08 & 0.000410 & 0.0194 & 3937.0 & 22.0 & $\mathrm{R}-450 \mathrm{~A}$ \\
\hline 171.4 & 0.18 & 0.08 & 0.000177 & 0.0195 & 2141.7 & 30.0 & $\mathrm{R}-450 \mathrm{~A}$ \\
\hline 177.0 & 0.22 & 0.08 & & & & 29.0 & $\mathrm{R}-450 \mathrm{~A}$ \\
\hline 184.0 & 0.31 & 0.08 & 0.000221 & 0.0195 & 2142.3 & 30.0 & $\mathrm{R}-450 \mathrm{~A}$ \\
\hline 175.2 & 0.09 & 0.08 & 0.000315 & 0.0195 & 4915.4 & 16.0 & $\mathrm{R}-450 \mathrm{~A}$ \\
\hline 182.2 & 0.22 & 0.08 & 0.000273 & 0.0195 & 4912.2 & 15.0 & $\mathrm{R}-450 \mathrm{~A}$ \\
\hline 188.2 & 0.33 & 0.08 & 0.000234 & & 4905.5 & 14.0 & $\mathrm{R}-450 \mathrm{~A}$ \\
\hline 192.6 & 0.41 & 0.08 & 0.000198 & 0.0195 & 4895.9 & 15.0 & $\mathrm{R}-450 \mathrm{~A}$ \\
\hline 195.2 & 0.49 & 0.08 & 0.000157 & 0.0194 & 4880.9 & 19.0 & $\mathrm{R}-450 \mathrm{~A}$ \\
\hline 194.0 & 0.53 & 0.08 & 0.000134 & 0.0194 & 4870.6 & 26.0 & $\mathrm{R}-450 \mathrm{~A}$ \\
\hline 168.4 & 0.60 & 0.07 & 0.000124 & 0.0190 & 4760.3 & 26.0 & $\mathrm{R}-450 \mathrm{~A}$ \\
\hline 192.9 & 0.63 & 0.07 & 0.000124 & 0.0190 & 4746.4 & 29.0 & $\mathrm{R}-450 \mathrm{~A}$ \\
\hline 155.4 & 0.07 & 0.08 & 0.000250 & 0.0195 & 5792.4 & 15.0 & $\mathrm{R}-450 \mathrm{~A}$ \\
\hline 163.7 & 0.17 & 0.08 & 0.000217 & 0.0195 & 5786.7 & 14.0 & $\mathrm{R}-450 \mathrm{~A}$ \\
\hline 171.5 & 0.26 & 0.08 & 0.000186 & 0.0195 & 5776.6 & 14.0 & $\mathrm{R}-450 \mathrm{~A}$ \\
\hline 178.1 & 0.33 & 0.08 & 0.000158 & 0.0195 & 5763.0 & 15.0 & $\mathrm{R}-450 \mathrm{~A}$ \\
\hline 183.4 & 0.39 & 0.08 & 0.000126 & 0.0194 & 5742.3 & 20.0 & $\mathrm{R}-450 \mathrm{~A}$ \\
\hline 184.1 & 0.42 & 0.08 & 0.000108 & 0.0194 & 5728.4 & 26.0 & $\mathrm{R}-450 \mathrm{~A}$ \\
\hline 174.1 & 0.49 & 0.07 & 0.000115 & 0.0190 & 5577.4 & 25.0 & $\mathrm{R}-450 \mathrm{~A}$ \\
\hline
\end{tabular}


Table A.3-1. (continued)

\begin{tabular}{|c|c|c|c|c|c|c|c|}
\hline $\mathrm{Nu}$ & $x_{\mathrm{q}}$ & $P_{\mathrm{r}}$ & Bo & $B_{\text {nd }}$ & $\mathrm{Re}$ & $U_{\mathrm{c}} / \%$ & fluid \\
\hline 6.0 & 0.52 & 07 & 00115 & 0.0190 & 5557.3 & 27.0 & 1 \\
\hline 192.3 & 0.11 & 0.08 & 0.000402 & 0.0195 & 3779.3 & 17.0 & $\mathrm{R}-450 \mathrm{~A}$ \\
\hline 196.6 & 0.28 & 0.08 & 0.000348 & 0.0195 & 3779.5 & 16.0 & $\mathrm{R}-450 \mathrm{~A}$ \\
\hline 200.4 & 0.41 & 0.08 & 0.000298 & 0.0195 & 3777.4 & 15.0 & $\mathrm{R}-450 \mathrm{~A}$ \\
\hline 203.0 & 0.52 & 0.08 & 0.000252 & 0.0195 & 3773.6 & 15.0 & $\mathrm{R}-450 \mathrm{~A}$ \\
\hline 204.4 & 0.62 & 0.08 & 0.000198 & 0.0194 & 3766.7 & 20.0 & $\mathrm{R}-450 \mathrm{~A}$ \\
\hline 203.9 & 0.66 & 0.08 & 0.000168 & 0.0194 & 3761.8 & 28.0 & $\mathrm{R}-450 \mathrm{~A}$ \\
\hline 181.1 & 0.10 & 0.08 & 0.000371 & 0.0195 & 2443.5 & 23.0 & $\mathrm{R}-450 \mathrm{~A}$ \\
\hline 183.0 & 0.26 & 0.08 & 0.000320 & 0.0195 & 2445.5 & 21.0 & $\mathrm{R}-450 \mathrm{~A}$ \\
\hline 3.7 & 0.38 & 0.08 & 73 & & 2446.6 & 21.0 & \\
\hline 182.6 & 0.48 & 0.08 & 0.000230 & 0.0195 & 2446.9 & 22.0 & $\mathrm{R}-450 \mathrm{~A}$ \\
\hline 178.5 & 0.57 & 0.08 & 0.000179 & 0.0195 & & 28.0 & $\mathrm{R}-450 \mathrm{~A}$ \\
\hline 157.0 & 0.06 & 0.08 & 0.000226 & 0.0197 & 6589.4 & 15.0 & $\mathrm{R}-450 \mathrm{~A}$ \\
\hline 167.0 & 0.16 & 0.08 & 96 & 0. & 2.2 & 15.0 & $\mathrm{R}-c$ \\
\hline 176.2 & 0.23 & 0.08 & 0.0 & 0.0197 & 0.1 & 14.0 & $\mathrm{R}-\mathrm{c}$ \\
\hline 184.7 & 0.30 & 0.08 & 0.0 & 0.0 & 6554.3 & 16.0 & $\mathrm{R}-450 \mathrm{~A}$ \\
\hline 192.6 & 0.35 & 0.08 & 0.000114 & 0.0195 & 6530.5 & 20.0 & $\mathrm{R}-450 \mathrm{~A}$ \\
\hline 195.0 & 0.38 & 0.08 & 0.0 & 95 & 4.6 & 26.0 & $\mathrm{R}-4$ \\
\hline 187.4 & 0.44 & 0.07 & 0.0 & 0.0191 & 1.6 & 25.0 & $\mathrm{R}-450 \mathrm{~A}$ \\
\hline 209.3 & 0.47 & 0.07 & & & & 27.0 & \\
\hline 185.5 & 0.09 & 0.08 & 0.000336 & 0.0195 & 4754.0 & 17.0 & $\mathrm{R}-450 \mathrm{~A}$ \\
\hline 192.5 & 0.24 & 0.08 & 0.000291 & 0.0195 & 4751.2 & 15.0 & $\mathrm{R}-450 \mathrm{~A}$ \\
\hline 198.2 & 0.35 & 0.08 & 0.000250 & 0.0195 & 4744.9 & 14.0 & $\mathrm{R}-450 \mathrm{~A}$ \\
\hline 202.6 & 0.44 & & & & & 15.0 & $\mathrm{R}-450 \mathrm{~A}$ \\
\hline 204.9 & 0.52 & 0.08 & 0.000166 & 0.0194 & 4721.6 & 20.0 & $\mathrm{R}-450 \mathrm{~A}$ \\
\hline 203.7 & 0.56 & 0.08 & 0.000141 & 0.0194 & 4712.0 & 28.0 & $\mathrm{R}-450 \mathrm{~A}$ \\
\hline 169.1 & 0.64 & 0.07 & 0.000125 & 0.0190 & 4607.5 & 27.0 & $\mathrm{R}-450 \mathrm{~A}$ \\
\hline 195.2 & 0.67 & 0.07 & 0.000125 & & & 29.0 & $\mathrm{R}-450 \mathrm{~A}$ \\
\hline 124.1 & 0.08 & 0.08 & 0.000057 & 0.0196 & 6739.5 & 26.0 & $\mathrm{R}-450 \mathrm{~A}$ \\
\hline 252.4 & 0.14 & 0.08 & 0.000163 & 0.0194 & 6713.0 & 19.0 & $\mathrm{R}-450 \mathrm{~A}$ \\
\hline 246.2 & 0.18 & 0.08 & 0.000178 & 0.0194 & 6711.1 & 18.0 & $\mathrm{R}-450 \mathrm{~A}$ \\
\hline 232.9 & 0.26 & 0.08 & 0.000204 & 0.0194 & 6699.6 & 17.0 & $\mathrm{R}-450 \mathrm{~A}$ \\
\hline 219.2 & 0.35 & 0.08 & 0.000227 & 0.0194 & 6679.4 & 17.0 & $\mathrm{R}-450 \mathrm{~A}$ \\
\hline 205.2 & 0.45 & 0.08 & 0.000252 & 0.0192 & 6648.7 & 17.0 & $\mathrm{R}-450 \mathrm{~A}$ \\
\hline 190.1 & 0.57 & 0.07 & 0.000278 & 0.0191 & 6602.9 & 18.0 & $\mathrm{R}-450 \mathrm{~A}$ \\
\hline 129.5 & 0.09 & 0.08 & 0.000075 & 0.0196 & 4900.2 & 30.0 & R-450A \\
\hline 126.7 & 0.11 & 0.08 & 0.000084 & 0.0196 & 4900.0 & 26.0 & $\mathrm{R}-450 \mathrm{~A}$ \\
\hline 250.1 & 0.19 & 0.08 & 0.000222 & 0.0194 & 4885.5 & 19.0 & $\mathrm{R}-450 \mathrm{~A}$ \\
\hline 246.2 & 0.24 & 0.08 & 0.000242 & 0.0194 & 4884.4 & 18.0 & $\mathrm{R}-450 \mathrm{~A}$ \\
\hline
\end{tabular}


Table A.3-1. (continued)

\begin{tabular}{|c|c|c|c|c|c|c|c|}
\hline $\mathrm{Nu}$ & $x_{\mathrm{q}}$ & $P_{\mathrm{r}}$ & Bo & $B_{\text {nd }}$ & $\mathrm{Re}$ & $U_{\mathrm{c}} / \%$ & fluid \\
\hline & 36 & 08 & .000276 & 0.0194 & 4878.8 & 18.0 & $\mathrm{R}-450 \mathrm{~A}$ \\
\hline 3.9 & 0.47 & 0.08 & 0.000309 & 0.0194 & 4869.5 & 18.0 & $\mathrm{R}-450 \mathrm{~A}$ \\
\hline 219.3 & 0.61 & 0.08 & 0.000342 & 0.0192 & 4855.6 & 19.0 & $\mathrm{R}-450 \mathrm{~A}$ \\
\hline 08.8 & 0.78 & 0.08 & 0.000378 & 0.0192 & & 21.0 & \\
\hline 33.9 & 0.11 & 0.08 & 0.000091 & 0.0196 & 3827.2 & 29.0 & $\mathrm{R}-450 \mathrm{~A}$ \\
\hline 1.4 & 0.18 & 0.08 & 0.000205 & 0.0194 & 3821.3 & 22.0 & $\mathrm{R}-450 \mathrm{~A}$ \\
\hline 229.3 & 0.23 & 0.08 & 0.000224 & 0.0194 & 3821.5 & 21.0 & $\mathrm{R}-450 \mathrm{~A}$ \\
\hline 24.1 & 0.34 & 0.08 & 0.000256 & 0.0194 & 3819.7 & 20.0 & $\mathrm{R}-450 \mathrm{~A}$ \\
\hline 17.5 & 0.44 & 0.08 & 0.000285 & 0.0194 & 3815.2 & 21.0 & $\mathrm{R}-450 \mathrm{~A}$ \\
\hline 209.9 & 0.57 & 0.08 & 0.000316 & 0.0194 & 7.8 & 22.0 & $\mathrm{R}-450 \mathrm{~A}$ \\
\hline 201.3 & 0.72 & 0.08 & 0.000350 & 0.0192 & 3796.3 & 23.0 & $\mathrm{R}-450 \mathrm{~A}$ \\
\hline 178.5 & 0.09 & 0.08 & 0.000275 & 0.0197 & 5855.7 & 17.0 & $\mathrm{R}-450 \mathrm{~A}$ \\
\hline 187.8 & 0.21 & 0.08 & 0.000 & 0.0 & 5850.3 & 15.0 & $\mathrm{R}-450 \mathrm{~A}$ \\
\hline 196.4 & 0.30 & 0.08 & 0.000204 & 0.0 & 5840.1 & 15.0 & $\mathrm{R}-450 \mathrm{~A}$ \\
\hline 203.6 & 0.37 & 0.08 & 0.000173 & 0.0195 & & 16.0 & $\mathrm{R}-450 \mathrm{~A}$ \\
\hline 209.1 & 0.44 & 0.08 & 0.000136 & 0.0194 & 5804.6 & 21.0 & $\mathrm{R}-450 \mathrm{~A}$ \\
\hline 209.5 & 0.48 & 0.08 & 0.000116 & 0.0 & 5790.2 & 28.0 & $\mathrm{R}-450 \mathrm{~A}$ \\
\hline 191.2 & 0.54 & 0.07 & 0.000123 & 0.0190 & 5632.9 & 25.0 & $\mathrm{R}-450 \mathrm{~A}$ \\
\hline 216.0 & 0.58 & 0.07 & & & & 28.0 & \\
\hline 132.7 & 0.04 & 0.08 & 0.000110 & 0.0194 & 49.7 & 23.0 & $\mathrm{R}-450 \mathrm{~A}$ \\
\hline 139.7 & 0.09 & 0.08 & 0.000097 & 0.0194 & 5.6 & 21.0 & $\mathrm{R}-450 \mathrm{~A}$ \\
\hline 146.1 & 0.13 & 0.08 & 0.000085 & 0.0194 & 6638.9 & 19.0 & $\mathrm{R}-450 \mathrm{~A}$ \\
\hline & 0.16 & 0.08 & & & & 20.0 & \\
\hline 156.7 & 0.19 & 0.08 & 0.00 & 0.0 & .2 & 27.0 & R-450A \\
\hline 158.5 & 0.11 & 0.08 & 0.000086 & 0.0197 & 5053.7 & 28.0 & $\mathrm{R}-450 \mathrm{~A}$ \\
\hline 311.8 & 0.19 & 0.08 & 0.000229 & 0.0197 & 5041.0 & 21.0 & $\mathrm{R}-450 \mathrm{~A}$ \\
\hline 306.4 & 0.25 & 0.08 & 0.000250 & & & 20.0 & $\mathrm{R}-450 \mathrm{~A}$ \\
\hline 294.8 & 0.37 & 0.08 & 0.000286 & 0.01 & & 19.0 & R-450A \\
\hline 282.2 & 0.49 & 0.08 & 0.000319 & 0.0195 & 5026.7 & 19.0 & $\mathrm{R}-450 \mathrm{~A}$ \\
\hline 268.5 & 0.63 & 0.08 & 0.000354 & 0.0195 & 5012.6 & 20.0 & $\mathrm{R}-450 \mathrm{~A}$ \\
\hline 253.3 & 0.80 & 0.08 & 0.000392 & 0.0194 & 91.4 & 21.0 & $\mathrm{R}-450 \mathrm{~A}$ \\
\hline 154.4 & 0.11 & 0.09 & 0.000082 & 0.0199 & 5310.3 & 28.0 & $\mathrm{R}-450 \mathrm{~A}$ \\
\hline 321.6 & 0.19 & 0.09 & 0.000229 & 0.0200 & 5296.8 & 21.0 & $\mathrm{R}-450 \mathrm{~A}$ \\
\hline 317.6 & 0.25 & 0.09 & 0.000250 & 0.0199 & 5296.9 & 20.0 & $\mathrm{R}-450 \mathrm{~A}$ \\
\hline 306.8 & 0.36 & 0.09 & 0.000286 & 0.0199 & 5292.8 & 19.0 & $\mathrm{R}-450 \mathrm{~A}$ \\
\hline 294.6 & 0.49 & 0.08 & 0.000320 & 0.0198 & 5284.3 & 19.0 & $\mathrm{R}-450 \mathrm{~A}$ \\
\hline 281.0 & 0.63 & 0.08 & 0.000354 & 0.0198 & 5270.4 & 19.0 & $\mathrm{R}-450 \mathrm{~A}$ \\
\hline 265.9 & 0.80 & 0.08 & 0.000392 & 0.0196 & 5249.0 & 20.0 & $\mathrm{R}-450 \mathrm{~A}$ \\
\hline 163.6 & 0.12 & 0.09 & 0.000084 & 0.0204 & 5148.1 & 29.0 & $\mathrm{R}-450 \mathrm{~A}$ \\
\hline
\end{tabular}


Table A.3-1. (continued)

\begin{tabular}{|c|c|c|c|c|c|c|c|}
\hline $\mathrm{Ju}$ & $x_{\mathrm{q}}$ & $P_{\mathrm{r}}$ & 0 & $B_{\text {nd }}$ & $\mathrm{Re}$ & $U_{\mathrm{c}} / \%$ & fluid \\
\hline & & & 9 & & & 22.0 & \\
\hline 1.6 & 26 & 09 & 0.000260 & 0.0202 & 5139.8 & 21.0 & $\mathrm{R}-450 \mathrm{~A}$ \\
\hline .5 & 38 & 09 & & 0.0202 & & 20.0 & $\mathrm{R}-450 \mathrm{~A}$ \\
\hline 8.7 & 0.51 & 0.09 & 0.000333 & 0.0202 & 5132.0 & 20.0 & R-450A \\
\hline 305.3 & 0.66 & 0.09 & .000369 & & & 20.0 & \\
\hline 0.5 & .83 & .09 & & & & 22.0 & $0 \mathrm{~A}$ \\
\hline & 13 & 08 & & & & 30.0 & \\
\hline .1 & 0.21 & 0.08 & 00194 & & & 9.0 & $\mathrm{OA}$ \\
\hline 2.8 & 0.26 & 0.08 & & & & 18.0 & \\
\hline 8.1 & 35 & 0.08 & & & & 7.0 & $\mathrm{DA}$ \\
\hline & 46 & .08 & & & & 7.0 & \\
\hline .1 & 0.58 & .08 & & & & 8.0 & \\
\hline .4 & 0.72 & .07 & & & & 18.0 & \\
\hline 12.7 & 0.17 & 0.10 & 75 & 0. & .1 & 28.0 & $3 \mathrm{~A}$ \\
\hline 7.9 & 0.19 & 0.10 & & & & 26.0 & \\
\hline 5.8 & 0.23 & .10 & & & & 24.0 & \\
\hline & 0 & & & & & 23.0 & \\
\hline 198.7 & 0.32 & 0.10 & 15 & 19 & 9.2 & 22.0 & $3 \mathrm{~A}$ \\
\hline 34.0 & 0.37 & 0.10 & & & & 23.0 & $\mathrm{R}-$ \\
\hline 6.1 & 0.12 & 0.10 & & & 6.5 & 29.0 & \\
\hline & & & & & & 28.0 & \\
\hline 04.0 & 0.16 & 10 & 54 & 0. & 6 & 26.0 & $13 \mathrm{~A}$ \\
\hline 193.3 & 0.19 & 0.10 & 0.0 & 0. & 2.3 & 25.0 & $13 \mathrm{~A}$ \\
\hline 181.2 & & & & & & 24.0 & \\
\hline 57.7 & & م 00 & & & & 24.0 & \\
\hline 187.1 & 0.08 & 10 & 7 & 0. & 12 & 30.0 & $3 \mathrm{~A}$ \\
\hline 184.9 & 0.10 & 0.10 & 0.000042 & 0.0 & 12952.6 & 28.0 & R-513A \\
\hline 178.8 & 0.12 & 0.10 & 0.0 & 0.0 & 29.5 & 27.0 & R-513A \\
\hline & & & & & & 27.0 & \\
\hline 59.8 & 0.17 & 0 & & & .2 & 28.0 & $3 \mathrm{~A}$ \\
\hline 174.1 & 0.12 & 0.10 & 0.000038 & 0.0 & 12997.6 & 30.0 & R-513A \\
\hline 163.7 & 0.14 & 0.10 & 0.000042 & & 12966.5 & 29.0 & R-513A \\
\hline 152.2 & 0.16 & 0.10 & & & & 28.0 & R-513A \\
\hline 185.8 & 0.11 & & & & & 30.0 & R-513A \\
\hline 177.7 & 0.13 & 0.10 & 0.000038 & 0.0221 & 13284.5 & 28.0 & R-513A \\
\hline 167.9 & 0.15 & 0.10 & 0.000042 & 0.0221 & 13251.9 & 26.0 & R-513A \\
\hline 156.7 & 0.17 & 0.10 & 0.000046 & 0.0219 & 13207.1 & 25.0 & R-513A \\
\hline & 0.15 & 0.10 & & & & 24.0 & R-513A \\
\hline 238.1 & 0.17 & 0.10 & 0.000092 & 0.0221 & 10037.1 & 23.0 & R-513A \\
\hline
\end{tabular}


Table A.3-1. (continued)

\begin{tabular}{|c|c|c|c|c|c|c|c|}
\hline $\mathrm{Nu}$ & $x_{\mathrm{q}}$ & $P_{\mathrm{r}}$ & Bo & $B_{\mathrm{nd}}$ & $\mathrm{Re}$ & $U_{\mathrm{c}} / \%$ & fluid \\
\hline 2 & 21 & 10 & 106 & 221 & & 21.0 & \\
\hline 1.1 & 0.26 & 0.10 & 0.000118 & 0.0221 & 9989.5 & 21.0 & R-513A \\
\hline 6.3 & 0.31 & .10 & .000130 & 0.0219 & 9948.6 & 21.0 & R-513A \\
\hline 180.5 & 0.38 & 0.10 & 0.000144 & 0.0217 & 9890.1 & 21.0 & R-513A \\
\hline 265.2 & 0.17 & 0.10 & 0.000093 & 0.0221 & & 23.0 & R-513A \\
\hline 62.0 & 0.19 & 0.10 & 0.000102 & 0.0221 & & 22.0 & $\mathrm{R}-513 \mathrm{~A}$ \\
\hline & 0.24 & 0.10 & & & & 21.0 & \\
\hline 236.9 & 0.29 & 0.10 & 0.000130 & 0.0221 & 9806.5 & 20.0 & R-513A \\
\hline 221.4 & 0.35 & 0.10 & 0.000144 & & & 20.0 & $3 \mathrm{~A}$ \\
\hline 204.2 & 0.42 & 0.10 & 0.000159 & 0.0 & & 21.0 & $3 \mathrm{~A}$ \\
\hline 3.5 & 0.18 & 0.10 & 0092 & & & 23.0 & \\
\hline 273.2 & 0.21 & 0.10 & 0.000101 & 0.0 & 0.0 & 22.0 & $3 \mathrm{~A}$ \\
\hline 265.1 & 0.26 & 0.10 & 15 & & & 20.0 & $3 \mathrm{~A}$ \\
\hline 252.3 & 0.31 & 0.10 & 0.000128 & 0.0 & 6.9 & 20.0 & R-513A \\
\hline 36.5 & 0.36 & 0.10 & 0.0 & & & 19.0 & $3 \mathrm{~A}$ \\
\hline 18.5 & 0.44 & 0.10 & 0.0 & & & 20.0 & $3 \mathrm{~A}$ \\
\hline 79.1 & 0.20 & 0.10 & & & & 23.0 & \\
\hline 277.9 & 0.22 & 0.10 & 0.000110 & 0.0223 & 9592.1 & 22.0 & $\mathrm{R}-513 \mathrm{~A}$ \\
\hline 269.2 & 0.28 & 0.10 & 0.0 & 0. & 9.9 & 20.0 & R-513A \\
\hline 255.4 & 0.33 & 0.10 & 0.0 & 0. & & 20.0 & $3 \mathrm{~A}$ \\
\hline 39.4 & 0.39 & 0.10 & & & & 19.0 & \\
\hline 220.9 & 0.47 & 0.10 & 0.00 & 0.0 & 9434.8 & 20.0 & R-513A \\
\hline 258.8 & 0.19 & 0.10 & 0.000 & 0.0 & 9731.8 & 23.0 & R-513A \\
\hline 255.0 & 0.21 & 0.10 & & & & 21.0 & \\
\hline 43.2 & 0.26 & 0.10 & 0.000120 & & 9702.9 & 20.0 & R-513A \\
\hline 229.2 & 0.31 & 0.10 & 0.00 & 0.0 & 9671.7 & 20.0 & $\mathrm{R}-513 \mathrm{~A}$ \\
\hline 213.6 & 0.37 & 0.10 & 0.000148 & 0.0219 & 9628.2 & 20.0 & R-513A \\
\hline 196.8 & 0.45 & 0.10 & 0.000164 & 0.0219 & & 20.0 & R-513A \\
\hline 187.4 & 0.18 & 0.10 & 0.000070 & & & 29.0 & R-513A \\
\hline 268.3 & 0.24 & 0.10 & 0. & 0. & 5.8 & 22.0 & $\mathrm{R}-513 \mathrm{~A}$ \\
\hline 265.4 & 0.27 & 0.10 & 0.000149 & 0.0223 & 7651.0 & 21.0 & R-513A \\
\hline 255.6 & 0.34 & 0.10 & 0.000170 & 0.0223 & 7635.9 & 20.0 & R-513A \\
\hline 43.6 & 0.42 & 0.10 & 0.000190 & 0.0223 & & 20.0 & R-513A \\
\hline 230.0 & 0.50 & 0.10 & 0.000210 & 0.0221 & 7583.5 & 20.0 & R-513A \\
\hline 214.6 & 0.60 & 0.10 & 0.000232 & 0.0219 & 7540.2 & 21.0 & R-513A \\
\hline 251.5 & 0.14 & 0.11 & 0.000352 & 0.0230 & 8096.8 & 22.0 & R-513A \\
\hline 241.7 & 0.29 & 0.11 & 0.000287 & 0.0228 & 8076.1 & 19.0 & R-513A \\
\hline 26.2 & 0.40 & 0.11 & 0.000227 & 0.0228 & 8053.5 & 17.0 & R-513A \\
\hline 203.6 & 0.47 & 0.11 & 0.000172 & 0.0228 & 8029.8 & 19.0 & R-513A \\
\hline
\end{tabular}


Table A.3-1. (continued)

\begin{tabular}{|c|c|c|c|c|c|c|c|}
\hline $\mathrm{Nu}$ & $x_{\mathrm{q}}$ & $P_{\mathrm{r}}$ & Bo & $B_{\text {nd }}$ & $\mathrm{Re}$ & $U_{\mathrm{c}} / \%$ & fluid \\
\hline & 60 & 10 & 0.000057 & 0.0223 & 7811.5 & 26.0 & 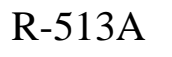 \\
\hline 110.7 & 0.61 & 0.10 & 0.000056 & 0.0221 & 7791.2 & 30.0 & R-513A \\
\hline 8.7 & 14 & .11 & 0.000064 & .0227 & & 28.0 & R-513A \\
\hline 72.3 & 0.18 & 0.11 & 0.000109 & 0.0225 & 235.3 & 21.0 & $3 \mathrm{~A}$ \\
\hline 70.4 & .21 & 0.11 & 0.000119 & 0.0225 & 10227.2 & 20.0 & \\
\hline 50.4 & 27 & 0.10 & & & & 19.0 & \\
\hline 7.0 & 33 & 0.10 & & & & 19.0 & $3 \mathrm{~A}$ \\
\hline 31.1 & 0.40 & 0.10 & & & & 19.0 & \\
\hline 3 & .48 & 0.10 & 185 & 0.02 & .3 & 19.0 & $3 \mathrm{~A}$ \\
\hline & .12 & 0.11 & & & & 28.0 & \\
\hline 3 & .17 & 0.10 & 07 & & & 21.0 & $3 \mathrm{~A}$ \\
\hline 62 & 0.19 & 0.10 & & & & 20.0 & \\
\hline 0.9 & 0.25 & 0.10 & 33 & 0.0 & 3 & 19.0 & $3 \mathrm{~A}$ \\
\hline 7 & 0.31 & 0.10 & & & & 19.0 & \\
\hline & 37 & .10 & & & & & \\
\hline 8 & 0.46 & 0.10 & & & & 19.0 & \\
\hline t & 0.11 & 0.11 & & & & 29.0 & $3 \mathrm{~A}$ \\
\hline 0 & 1 & 0.10 & & & & 21.0 & \\
\hline & & & & & & & \\
\hline 9 & 0.23 & .10 & 29 & 3 & & 19.0 & \\
\hline 1.8 & 0.29 & 0.10 & 4 & & & 19.0 & $3 \mathrm{~A}$ \\
\hline 8.8 & 0.35 & 0.10 & 60 & 21 & & 19.0 & \\
\hline & & & & & & & \\
\hline 146.7 & 0.13 & 0 & & & & 29.0 & \\
\hline 269.9 & 0.18 & 0.10 & 20 & 23 & 0.0 & 21.0 & $3 \mathrm{~A}$ \\
\hline 63.0 & 0.21 & 0.10 & 30 & 223 & 3.2 & 20.0 & $3 \mathrm{~A}$ \\
\hline & & & & & & 19.0 & $3 \mathrm{~A}$ \\
\hline 101 & 0.33 & 10 & 6 & & & 18.0 & $3 \mathrm{~A}$ \\
\hline 14.6 & 0.41 & 0.10 & 84 & 19 & 9.2 & 19.0 & $\mathrm{R}-513 \mathrm{~A}$ \\
\hline 196.8 & 0.50 & 0.10 & 0.00 & 0.0217 & 9460.9 & 19.0 & $\mathrm{R}-513 \mathrm{~A}$ \\
\hline & 0.16 & 0.11 & & & & 29.0 & \\
\hline 90.0 & 0.21 & 0.10 & & & & 21.0 & $\mathrm{R}-513 \mathrm{~A}$ \\
\hline 287.0 & 0.24 & 0.10 & 0.00 & $0 .($ & 9743.4 & 20.0 & $\mathrm{R}-513 \mathrm{~A}$ \\
\hline 274.9 & 0.30 & 0.10 & 0.000152 & 0.0223 & 9717.4 & 19.0 & $\mathrm{R}-513 \mathrm{~A}$ \\
\hline & 0.37 & 0.10 & 0.000170 & 0.0221 & 9680.6 & 19.0 & R-513A \\
\hline & 0.45 & 0.10 & & & & 19.0 & $\mathrm{R}-513 \mathrm{~A}$ \\
\hline 222.4 & 0.54 & 0.10 & 0.000208 & 0.0219 & 9557.1 & 19.0 & R-513A \\
\hline & 0.22 & 0.10 & 0.000060 & 0.0223 & & 30.0 & $\mathrm{R}-513 \mathrm{~A}$ \\
\hline 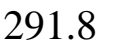 & 0.28 & 0.10 & 0.000165 & 0.0223 & 7080.6 & 22.0 & R-513A \\
\hline
\end{tabular}


Table A.3-1. (continued)

\begin{tabular}{|c|c|c|c|c|c|c|c|}
\hline $\mathrm{Nu}$ & $x_{\mathrm{q}}$ & $P_{\mathrm{r}}$ & Bo & $B_{\text {nd }}$ & $\mathrm{Re}$ & $U_{\mathrm{c}} / \%$ & fluid \\
\hline 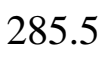 & 32 & 10 & .000180 & 0.0221 & 7075.5 & 21.0 & 1 \\
\hline 1.5 & 0.40 & 0.10 & 0.000205 & 0.0221 & 7060.2 & 20.0 & R-513A \\
\hline 256.6 & 0.49 & 0.10 & 0.000229 & 0.0221 & 7038.2 & 20.0 & R-513A \\
\hline 240.8 & 0.59 & 0.10 & 0.000253 & 0.0219 & 7007.5 & 21.0 & R-513A \\
\hline 223.7 & 0.72 & 0.10 & 0.000280 & 0.0219 & 6964.1 & 21.0 & R-513A \\
\hline 187.2 & 0.21 & 0.10 & 0.000078 & 0.0223 & 7244.2 & 28.0 & $\mathrm{R}-5$ \\
\hline 286.3 & 0.28 & 0.10 & 0.000163 & 0.0223 & 7208.1 & 21.0 & R-513A \\
\hline 283.6 & 0.32 & 0.10 & 0.000178 & 0.0223 & 7202.3 & 21.0 & R-513A \\
\hline 274.4 & 0.40 & 0.10 & 0.000203 & 0.0221 & 7186.1 & 20.0 & R-513A \\
\hline 62.6 & 0.49 & 0.10 & 0.000226 & 0.0221 & 7163.8 & 20.0 & R-513A \\
\hline 248.9 & 0.59 & 0.10 & 0.000250 & 0.0219 & 7133.0 & 21.0 & R-513A \\
\hline 233.0 & 0.71 & 0.10 & 0.000277 & 0.0219 & 7090.2 & 22.0 & \\
\hline 263.2 & 0.21 & 0.10 & 0.000401 & 0.0221 & 6198.9 & 21.0 & $\mathrm{R}-513 \mathrm{~A}$ \\
\hline 50.3 & 0.38 & 0.10 & 0.000331 & 0.0219 & 6181.2 & 19.0 & R-513A \\
\hline 233.5 & 0.50 & 0.10 & 0.000265 & 0.0 & 61 & 17.0 & $\mathrm{R}-$ \\
\hline 211.8 & 0.59 & 0.10 & 0.000205 & 0.0219 & 6146.5 & 19.0 & $\mathrm{R}-513 \mathrm{~A}$ \\
\hline 189.1 & 0.33 & 0.07 & 0.000223 & 0.0190 & 4108.2 & 23.0 & $R-515 B$ \\
\hline 193.7 & 0.42 & 0.07 & 0.000189 & 0.0188 & 4095.4 & 22.0 & R-515B \\
\hline 196.9 & 0.49 & 0.07 & & & & 23.0 & \\
\hline 198.7 & 0.55 & 0.07 & 0.000129 & 0.0188 & 4070.4 & 27.0 & R-515B \\
\hline 174.6 & 0.10 & 0.08 & 0.000338 & 0.0196 & 5553.1 & 22.0 & R-515B \\
\hline 177.3 & 0.24 & 0.08 & 0.000281 & 0.0196 & 5537.5 & 20.0 & R-515B \\
\hline 177.5 & 0.35 & 0.08 & & & & 18.0 & \\
\hline 173.2 & 0.43 & 0.08 & 0.000179 & 0.0194 & 5500.6 & 20.0 & R-515B \\
\hline 222.6 & 0.15 & 0.07 & 0.000386 & 0.0192 & 4749.5 & 25.0 & R-515B \\
\hline 215.9 & 0.31 & 0.07 & 0.000319 & 0.0190 & 4735.7 & 22.0 & R-515B \\
\hline 205.2 & 0.43 & 0.07 & & & & 21.0 & \\
\hline 189.7 & 0.52 & 0.07 & 0.000198 & 0.0191 & 4707.1 & 22.0 & R-515B \\
\hline 87.5 & 0.66 & 0.07 & 0.000066 & 0.0187 & 4569.7 & 28.0 & R-515B \\
\hline 153.7 & 0.17 & 0.07 & 0.000134 & 0.0188 & 5036.2 & 27.0 & R-515B \\
\hline 159.2 & 0.22 & 0.07 & 0.000113 & 0.0188 & 5024.3 & 27.0 & R-515B \\
\hline 163.7 & 0.27 & 0.07 & 0.000094 & 0.0188 & 5012.1 & 28.0 & R-515B \\
\hline 218.5 & 0.21 & 0.07 & 0.000093 & 0.0187 & 6243.5 & 29.0 & R-515B \\
\hline 196.2 & 0.25 & 0.07 & 0.000103 & 0.0186 & 6223.3 & 26.0 & R-515B \\
\hline 175.3 & 0.30 & 0.06 & 0.000114 & 0.0186 & 6194.5 & 25.0 & $R-515 B$ \\
\hline 155.1 & 0.36 & 0.06 & 0.000126 & 0.0184 & 6153.3 & 24.0 & R-515B \\
\hline 304.9 & 0.23 & 0.07 & 0.000175 & 0.0189 & 5518.9 & 27.0 & $R-515 B$ \\
\hline 290.8 & 0.27 & 0.07 & 0.000190 & 0.0189 & 5514.6 & 25.0 & R-515B \\
\hline 263.8 & 0.36 & 0.07 & 0.000217 & 0.0188 & 5498.7 & 24.0 & R-515B \\
\hline
\end{tabular}


Table A.3-1. (continued)

$\begin{array}{cccccccc}\mathrm{Nu} & x_{\mathrm{q}} & P_{\mathrm{r}} & \mathrm{B} 0 & B_{\mathrm{nd}} & \mathrm{Re} & U_{\mathrm{c}} \% & \text { fluid } \\ & & & & & & & \\ 238.3 & 0.46 & 0.07 & 0.000243 & 0.0188 & 5473.6 & 23.0 & \mathrm{R}-515 \mathrm{~B} \\ 214.3 & 0.56 & 0.07 & 0.000268 & 0.0187 & 5437.2 & 23.0 & \mathrm{R}-515 \mathrm{~B} \\ 190.7 & 0.70 & 0.07 & 0.000296 & 0.0186 & 5384.4 & 23.0 & \mathrm{R}-515 \mathrm{~B} \\ 340.8 & 0.28 & 0.07 & 0.000197 & 0.0188 & 5037.0 & 26.0 & \mathrm{R}-515 \mathrm{~B} \\ 327.5 & 0.33 & 0.07 & 0.000214 & 0.0188 & 5031.7 & 25.0 & \mathrm{R}-515 \mathrm{~B} \\ 299.5 & 0.43 & 0.07 & 0.000245 & 0.0187 & 5015.0 & 23.0 & \mathrm{R}-515 \mathrm{~B} \\ 272.4 & 0.54 & 0.07 & 0.000273 & 0.0187 & 4990.7 & 22.0 & \mathrm{R}-515 \mathrm{~B} \\ 246.1 & 0.66 & 0.07 & 0.000302 & 0.0186 & 4956.5 & 22.0 & \mathrm{R}-515 \mathrm{~B} \\ 219.5 & 0.81 & 0.06 & 0.000334 & 0.0185 & 4908.1 & 21.0 & \mathrm{R}-515 \mathrm{~B} \\ 292.5 & 0.17 & 0.07 & 0.000153 & 0.0192 & 6319.2 & 25.0 & \mathrm{R}-515 \mathrm{~B} \\ 279.0 & 0.21 & 0.07 & 0.000167 & 0.0192 & 6315.8 & 23.0 & \mathrm{R}-515 \mathrm{~B} \\ 252.5 & 0.28 & 0.07 & 0.000191 & 0.0190 & 6299.0 & 21.0 & \mathrm{R}-515 \mathrm{~B} \\ 227.6 & 0.37 & 0.07 & 0.000213 & 0.0191 & 6270.8 & 21.0 & \mathrm{R}-515 \mathrm{~B} \\ 203.7 & 0.46 & 0.07 & 0.000235 & 0.0189 & 6228.6 & 20.0 & \mathrm{R}-515 \mathrm{~B} \\ 180.4 & 0.58 & 0.07 & 0.000260 & 0.0188 & 6166.4 & 20.0 & \mathrm{R}-515 \mathrm{~B} \\ 276.8 & 0.12 & 0.08 & 0.000121 & 0.0193 & 7884.4 & 24.0 & \mathrm{R}-515 \mathrm{~B} \\ 268.3 & 0.15 & 0.08 & 0.000132 & 0.0193 & 7881.5 & 22.0 & \mathrm{R}-515 \mathrm{~B} \\ 245.9 & 0.21 & 0.08 & 0.000151 & 0.0193 & 7861.6 & 21.0 & \mathrm{R}-515 \mathrm{~B} \\ 123.6 & 0.13 & 0.08 & 0.000103 & 0.0194 & 7231.8 & 23.0 & \mathrm{R}-450 \mathrm{~A} \\ 221.7 & 0.28 & 0.07 & 0.000169 & 0.0192 & 7826.1 & 20.0 & \mathrm{R}-515 \mathrm{~B} \\ 134.6 & 0.16 & 0.08 & 0.000113 & 0.0194 & 7230.2 & 21.0 & \mathrm{R}-450 \mathrm{~A}\end{array}$


Table A.3-1. (continued)

\begin{tabular}{|c|c|c|c|c|c|c|c|}
\hline $\mathrm{Nu}$ & $x_{\mathrm{q}}$ & $P_{\mathrm{r}}$ & Bo & $B_{\mathrm{nd}}$ & $\mathrm{Re}$ & $U_{\mathrm{c}} / \%$ & fluid \\
\hline 206.9 & 0.21 & 0.08 & 00129 & 0.0194 & 19.1 & 20.0 & $\mathrm{R}$ \\
\hline 194.7 & 0.27 & 0.08 & 0.000144 & 0.0194 & 7199.2 & 20.0 & $\mathrm{R}-450 \mathrm{~A}$ \\
\hline 181.2 & 0.33 & 0.08 & 0.000159 & 0.0193 & 7168.7 & 20.0 & $\mathrm{R}-450 \mathrm{~A}$ \\
\hline 166.7 & 0.41 & 0.07 & 0.000176 & 0.0191 & 7123.2 & 21.0 & $\mathrm{R}-450 \mathrm{~A}$ \\
\hline 126.0 & 0.13 & 0.08 & 0.000053 & 0.0196 & 6630.2 & 29.0 & $\mathrm{R}-450 \mathrm{~A}$ \\
\hline 239.0 & 0.18 & 0.08 & 0.000142 & 0.0194 & 6603.5 & 21.0 & $\mathrm{R}-450 \mathrm{~A}$ \\
\hline 233.7 & 0.21 & 0.08 & 0.000154 & 0.0194 & 6602.0 & 20.0 & $\mathrm{R}-450 \mathrm{~A}$ \\
\hline 220.9 & 0.29 & 0.08 & 0.000176 & 0.0194 & 6590.6 & 20.0 & $\mathrm{R}-450 \mathrm{~A}$ \\
\hline 207.0 & 0.36 & 0.08 & 0.000197 & 0.0192 & 6569.6 & 19.0 & $\mathrm{R}-450 \mathrm{~A}$ \\
\hline 192.1 & 0.45 & 0.08 & 0.000218 & 0.0192 & 6537.0 & 20.0 & $\mathrm{R}-450 \mathrm{~A}$ \\
\hline 176.1 & 0.56 & 0.07 & 0.000 & & & 20.0 & $50 \mathrm{~A}$ \\
\hline 209.2 & 0.09 & 0.08 & 0.000059 & 0.0193 & 8808.1 & 27.0 & $\mathrm{R}-450 \mathrm{~A}$ \\
\hline 209.7 & 0.10 & 0.08 & 0.000065 & 0.01 & 8806.5 & 26.0 & $\mathrm{R}-450 \mathrm{~A}$ \\
\hline 203.9 & 0.13 & 0.08 & 0.000074 & 0.0191 & 8794.8 & 25.0 & $\mathrm{R}-450 \mathrm{~A}$ \\
\hline 193.6 & 0.17 & 0.07 & 0.000082 & 0.0 & 8773.9 & 24.0 & $\mathrm{R}-450 \mathrm{~A}$ \\
\hline 181.0 & 0.21 & 0.07 & 0.000 & 0.0 & 8742.0 & 24.0 & $\mathrm{R}-\mathrm{C}$ \\
\hline 166.1 & 0.25 & 0.07 & 0.0 & 0. & & 24.0 & \\
\hline 186.6 & 0.15 & 0.07 & 0.000054 & 0.0189 & 8255.8 & 30.0 & R-450A \\
\hline 171.9 & 0.17 & 0.07 & 0.000060 & 0.0189 & 8234.8 & 27.0 & $\mathrm{R}-450 \mathrm{~A}$ \\
\hline 156.9 & 0.20 & 0.07 & 0.000066 & 0.0188 & 8204.8 & 26.0 & $\mathrm{R}-450 \mathrm{~A}$ \\
\hline 141.6 & 0.23 & 0.07 & 0.000 & 0 . & 8162.2 & 25.0 & $\mathrm{R}-450 \mathrm{~A}$ \\
\hline 203.5 & 0.28 & 0.09 & 0.000103 & 0.0210 & 4653.4 & 30.0 & $\mathrm{R}-134 \mathrm{a}$ \\
\hline 254.4 & 0.35 & 0.09 & 0.000170 & 0.0208 & 4639.4 & 23.0 & $\mathrm{R}-134 \mathrm{a}$ \\
\hline 252.0 & 0.40 & 0.09 & 0.000185 & & 4637.2 & 21.0 & R-134a \\
\hline 245.2 & 0.48 & 0.09 & 0.000211 & 0.0208 & 4631.3 & 20.0 & R-134a \\
\hline 237.0 & 0.57 & 0.08 & 0.000236 & 0.0208 & 4623.4 & 20.0 & $\mathrm{R}-134 \mathrm{a}$ \\
\hline 227.5 & 0.67 & 0.08 & 0.000261 & 0.0208 & 4612.7 & 21.0 & $\mathrm{R}-134 \mathrm{a}$ \\
\hline 216.8 & 0.80 & 0.08 & 0.000289 & 0.0207 & 4598.0 & 22.0 & $\mathrm{R}-134 \mathrm{a}$ \\
\hline 181.0 & 0.13 & 0.09 & 0.000075 & 0.0210 & 6514.1 & 28.0 & R-134a \\
\hline 260.4 & 0.18 & 0.08 & 0.000144 & 0.0208 & 6482.6 & 20.0 & $\mathrm{R}-134 \mathrm{a}$ \\
\hline 258.5 & 0.22 & 0.08 & 0.000157 & 0.0208 & 6477.9 & 19.0 & $\mathrm{R}-134 \mathrm{a}$ \\
\hline 249.8 & 0.29 & 0.08 & 0.000179 & 0.0208 & 6464.3 & 18.0 & $\mathrm{R}-134 \mathrm{a}$ \\
\hline 238.2 & 0.37 & 0.08 & 0.000200 & 0.0207 & 6445.3 & 18.0 & $\mathrm{R}-134 \mathrm{a}$ \\
\hline 224.5 & 0.46 & 0.08 & 0.000221 & 0.0207 & 6419.3 & 18.0 & R-134a \\
\hline 209.1 & 0.57 & 0.08 & 0.000245 & 0.0205 & 6382.7 & 19.0 & $\mathrm{R}-134 \mathrm{a}$ \\
\hline 254.9 & 0.20 & 0.08 & 0.000178 & 0.0208 & 4418.1 & 24.0 & $\mathrm{R}-134 \mathrm{a}$ \\
\hline 251.9 & 0.25 & 0.08 & 0.000194 & 0.0208 & 4415.7 & 22.0 & $\mathrm{R}-134 \mathrm{a}$ \\
\hline 244.4 & 0.34 & 0.08 & 0.000222 & 0.0208 & 4409.6 & 21.0 & $\mathrm{R}-134 \mathrm{a}$ \\
\hline 236.2 & 0.43 & 0.08 & 0.000247 & 0.0208 & 4401.6 & 21.0 & R-134a \\
\hline
\end{tabular}


Table A.3-1. (continued)

\begin{tabular}{|c|c|c|c|c|c|c|c|}
\hline $\mathrm{Nu}$ & $x_{\mathrm{q}}$ & $P_{\mathrm{r}}$ & Bo & $B_{\text {nd }}$ & $\mathrm{Re}$ & $U_{\mathrm{c}} / \%$ & fluid \\
\hline 7.4 & 0.54 & .08 & 0.000274 & 0.0207 & 4391.2 & 22.0 & R-134a \\
\hline 17.4 & 0.67 & 0.08 & 0.000303 & 0.0207 & 4376.9 & 24.0 & R-134a \\
\hline 189.8 & 0.10 & 0.09 & 0.000080 & 0.0210 & 6100.5 & 30.0 & R-134a \\
\hline 272.5 & 0.16 & 0.09 & 0.000156 & 0.0208 & 6072.8 & 21.0 & R-134a \\
\hline 269.1 & 0.20 & 0.09 & 0.000170 & 0.0208 & 6068.4 & 20.0 & R-134a \\
\hline 259.6 & 0.28 & 0.08 & 0.000194 & 0.0208 & 6056.9 & 19.0 & R-134a \\
\hline 248.4 & 0.36 & 0.08 & 0.000217 & 0.0208 & 6041.2 & 19.0 & R-134a \\
\hline 235.8 & 0.46 & 0.08 & 0.000240 & 0.0207 & 6019.9 & 19.0 & R-134a \\
\hline 221.6 & 0.57 & 0.08 & 0.000265 & 0.0207 & 5990.3 & 20.0 & R-134a \\
\hline 230.6 & 0.21 & 0.09 & 0.000179 & 0.0208 & 3728.4 & 25.0 & R-134a \\
\hline 227.8 & 0.25 & 0.09 & 0.000195 & 0.0208 & 3726.8 & 24.0 & R-134a \\
\hline 221.7 & 0.34 & 0.08 & 0.000223 & 0.0208 & 3723.1 & 22.0 & R-134a \\
\hline 215.4 & 0.44 & 0.08 & 0.000249 & 0.0208 & 3718.5 & 23.0 & R-134a \\
\hline 208.3 & 0.55 & 0.08 & 0.000275 & 0.0208 & 3712.4 & 23.0 & R-134a \\
\hline 200.6 & 0.68 & 0.08 & 0.000305 & 0.0208 & 3704.4 & 25.0 & R-134a \\
\hline 201.5 & 0.31 & 0.08 & 0.000189 & 0.0208 & 2590.4 & 29.0 & R-134a \\
\hline 197.6 & 0.39 & 0.08 & 0.000211 & 0.0208 & 2589.1 & 28.0 & R-134a \\
\hline 193.6 & 0.48 & 0.08 & 0.000233 & 0.0208 & 2587.4 & 29.0 & R-134a \\
\hline 189.5 & 0.59 & 0.08 & 0.000258 & 0.0207 & 2585.3 & 30.0 & R-134a \\
\hline 162.1 & 0.06 & 0.09 & & & & 17.0 & \\
\hline 171.9 & 0.17 & 0.09 & 0.000217 & 0.0210 & 4482.3 & 17.0 & $\mathrm{R}-134 \mathrm{a}$ \\
\hline 182.3 & 0.25 & 0.09 & 0.000196 & 0.0210 & 4476.3 & 16.0 & R-134a \\
\hline 193.0 & 0.33 & 0.09 & 0.000177 & 0.0210 & 4469.6 & 17.0 & R-134a \\
\hline 207.4 & 0.40 & 0.09 & 0.000155 & & & 21.0 & R-134a \\
\hline 216.3 & 0.44 & 0.09 & 0.000143 & 0.0208 & 4454.3 & 25.0 & R-134a \\
\hline 193.4 & 0.08 & 0.09 & 0.000276 & 0.0210 & 3457.3 & 20.0 & R-134a \\
\hline 201.3 & 0.20 & 0.09 & 0.000249 & 0.0210 & 3454.3 & 19.0 & R-134a \\
\hline 209.6 & 0.30 & 0.09 & 0.000225 & 0.0210 & 3450.8 & 20.0 & $\mathrm{R}-134 \mathrm{a}$ \\
\hline 218.8 & 0.38 & 0.09 & 0.000202 & 0.0210 & 3447.0 & 21.0 & $\mathrm{R}-134 \mathrm{a}$ \\
\hline 231.6 & 0.46 & 0.09 & 0.000176 & 0.0208 & 3441.9 & 25.0 & R-134a \\
\hline 240.4 & 0.50 & 0.09 & 0.000162 & 0.0208 & 3438.7 & 29.0 & R-134a \\
\hline 158.8 & 0.05 & 0.09 & 0.000167 & 0.0208 & 6618.6 & 19.0 & R-134a \\
\hline 170.4 & 0.12 & 0.08 & 0.000151 & 0.0208 & 6607.9 & 18.0 & R-134a \\
\hline 181.8 & 0.18 & 0.08 & 0.000136 & 0.0208 & 6594.4 & 18.0 & R-134a \\
\hline 192.8 & 0.24 & 0.08 & 0.000122 & 0.0208 & 6579.0 & 19.0 & R-134a \\
\hline 205.4 & 0.29 & 0.08 & 0.000106 & 0.0207 & 6557.3 & 22.0 & $\mathrm{R}-134 \mathrm{a}$ \\
\hline 211.8 & 0.31 & 0.08 & 0.000097 & 0.0207 & 6543.6 & 26.0 & R-134a \\
\hline 227.5 & 0.38 & 0.08 & 0.000124 & 0.0204 & 6400.3 & 27.0 & R-134a \\
\hline 244.5 & 0.41 & 0.08 & 0.000124 & 0.0203 & 6380.2 & 28.0 & R-134a \\
\hline
\end{tabular}


Table A.3-1. (continued)

\begin{tabular}{|c|c|c|c|c|c|c|c|}
\hline $\mathrm{Nu}$ & $x_{\mathrm{q}}$ & $P_{\mathrm{r}}$ & Bo & $B_{\text {nd }}$ & $\mathrm{Re}$ & $U_{\mathrm{c}} / \%$ & fluid \\
\hline 0.4 & 07 & .09 & 0.000233 & 0.0210 & 2561.5 & 25.0 & $134 \mathrm{a}$ \\
\hline 158.4 & 0.17 & 0.09 & 0.000215 & 0.0210 & 2560.0 & 23.0 & R-134a \\
\hline 167.5 & 0.25 & 0.09 & 0.000199 & 0.0210 & 2558.6 & 23.0 & $\mathrm{R}-134 \mathrm{a}$ \\
\hline 177.7 & 0.33 & 0.09 & 0.000183 & 0.0210 & 2557.1 & 25.0 & R-134a \\
\hline 193.3 & 0.40 & 0.09 & 0.000166 & 0.0208 & 2555.2 & 29.0 & R-134a \\
\hline 167.3 & 0.06 & 0.09 & 0.000231 & 0.0210 & 4564.3 & 18.0 & R-134a \\
\hline 176.3 & 0.16 & 0.09 & 0.000209 & 0.0210 & 4559.1 & 17.0 & R-134a \\
\hline 185.7 & 0.25 & 0.09 & 0.000188 & 0.0208 & 4552.6 & 17.0 & R-134a \\
\hline 195.0 & 0.32 & 0.09 & 0.000168 & 0.0208 & 4545.4 & 18.0 & R-134a \\
\hline 207.0 & 0.39 & 0.08 & 0.000146 & 0.0208 & 4535.4 & 22.0 & R-134a \\
\hline 213.9 & 0.42 & 0.08 & 0.000134 & 0.0208 & 4529.1 & 26.0 & R-134a \\
\hline 216.4 & 0.49 & 0.08 & 0.000141 & 0.0205 & 4464.3 & 30.0 & R-134a \\
\hline 165.4 & 0.06 & 0.09 & 0.000227 & 0.0210 & 4588.4 & 18.0 & R-134a \\
\hline 174.4 & 0.16 & 0.09 & 0.000204 & 0.0210 & 4583.2 & 17.0 & R-134a \\
\hline 184.0 & 0.24 & 0.09 & 0.000184 & 0.0208 & 4576.9 & 17.0 & R-134a \\
\hline 193.4 & 0.31 & 0.09 & 0.000165 & 0.0208 & 4569.7 & 18.0 & $\mathrm{R}-134 \mathrm{a}$ \\
\hline 205.6 & 0.38 & 0.08 & 0.000143 & 0.0208 & 4559.8 & 22.0 & R-134a \\
\hline 212.8 & 0.41 & 0.08 & 0.000131 & 0.0208 & 4553.6 & 26.0 & R-134a \\
\hline 160.8 & 0.06 & 0.09 & 0.000170 & 0.0210 & 6691.8 & 19.0 & R-134a \\
\hline 171.5 & 0.13 & 0.09 & & & & 18.0 & \\
\hline 181.9 & 0.19 & 0.09 & 0.000136 & 0.0208 & 6667.2 & 18.0 & $\mathrm{R}-134 \mathrm{a}$ \\
\hline 191.7 & 0.24 & 0.09 & 0.000122 & 0.0208 & 6651.6 & 18.0 & R-134a \\
\hline 202.4 & 0.30 & 0.08 & 0.000104 & 0.0208 & 6629.9 & 22.0 & R-134a \\
\hline 207.5 & 0.32 & 0.08 & 0.000095 & & & 26.0 & R-134a \\
\hline 223.9 & 0.39 & 0.08 & 0.000123 & 0.0204 & 6470.7 & 26.0 & R-134a \\
\hline 240.2 & 0.42 & 0.08 & 0.000122 & 0.0204 & 6450.0 & 28.0 & R-134a \\
\hline 160.3 & 0.06 & 0.09 & 0.000167 & 0.0210 & 6722.4 & 18.0 & R-134a \\
\hline 171.2 & 0.13 & 0.09 & 0.000150 & 0.0210 & 6711.0 & 18.0 & $\mathrm{R}-134 \mathrm{a}$ \\
\hline 181.6 & 0.19 & 0.09 & 0.000134 & 0.0208 & 6696.7 & 17.0 & $\mathrm{R}-134 \mathrm{a}$ \\
\hline 191.2 & 0.24 & 0.08 & 0.000120 & 0.0208 & 6680.5 & 18.0 & R-134a \\
\hline 201.4 & 0.29 & 0.08 & 0.000103 & 0.0208 & 6657.8 & 22.0 & R-134a \\
\hline 205.9 & 0.32 & 0.08 & 0.000094 & 0.0207 & 6643.5 & 25.0 & R-134a \\
\hline 220.8 & 0.38 & 0.08 & 0.000122 & 0.0204 & 6494.4 & 26.0 & R-134a \\
\hline 236.4 & 0.41 & 0.08 & 0.000121 & 0.0204 & 6473.6 & 28.0 & R-134a \\
\hline 261.0 & 0.47 & 0.08 & 0.000121 & 0.0203 & 6434.4 & 30.0 & R-134a \\
\hline 182.1 & 0.08 & 0.09 & 0.000277 & 0.0210 & 3425.8 & 20.0 & $\mathrm{R}-134 \mathrm{a}$ \\
\hline 189.9 & 0.20 & 0.09 & 0.000249 & 0.0210 & 3422.9 & 19.0 & R-134a \\
\hline 198.3 & 0.29 & 0.09 & 0.000224 & 0.0210 & 3419.5 & 19.0 & R-134a \\
\hline 207.2 & 0.38 & 0.09 & 0.000201 & 0.0210 & 3415.8 & 20.0 & R-134a \\
\hline
\end{tabular}


Table A.3-1. (continued)

\begin{tabular}{|c|c|c|c|c|c|c|c|}
\hline $\mathrm{Nu}$ & $x_{\mathrm{q}}$ & $P_{\mathrm{r}}$ & Bo & $B_{\text {nd }}$ & $\mathrm{Re}$ & $U_{\mathrm{c}} / \%$ & fluid \\
\hline 9.2 & 0.46 & 0.09 & 0.000174 & 0.0210 & 3410.8 & 24.0 & $134 \mathrm{a}$ \\
\hline 27.2 & 0.50 & 0.09 & 0.000159 & 0.0210 & 3407.6 & 29.0 & R-134a \\
\hline 149.0 & 0.07 & 0.08 & 0.000236 & 0.0208 & 2613.7 & 25.0 & $\mathrm{R}-134 \mathrm{a}$ \\
\hline 157.0 & 0.17 & 0.08 & 0.000218 & 0.0208 & 2612.1 & 23.0 & R-134a \\
\hline 166.0 & 0.26 & 0.08 & 0.000201 & 0.0208 & 2610.4 & 23.0 & R-134a \\
\hline 176.1 & 0.33 & 0.08 & 0.000186 & 0.0208 & 2608.7 & 24.0 & R-134a \\
\hline 191.2 & 0.41 & 0.08 & 0.000168 & 0.0208 & 2606.5 & 28.0 & R-134a \\
\hline 252.8 & 0.18 & 0.08 & 0.000169 & 0.0208 & 4663.7 & 23.0 & R-134a \\
\hline 249.5 & 0.23 & 0.08 & 0.000184 & 0.0208 & 4661.2 & 22.0 & R-134a \\
\hline 241.5 & 0.31 & 0.08 & 0.000211 & 0.0208 & 4654.7 & 20.0 & R-134a \\
\hline 233.1 & 0.40 & 0.08 & 0.000235 & 0.0208 & 4646.2 & 20.0 & R-134a \\
\hline 223.5 & 0.51 & 0.08 & 0.000261 & 0.0207 & 4634.8 & 21.0 & $\mathrm{R}-134 \mathrm{a}$ \\
\hline 213.0 & 0.63 & 0.08 & 0.000288 & 0.0207 & 4619.2 & 22.0 & R-134a \\
\hline 181.2 & 0.10 & 0.09 & 0.000076 & 0.0208 & 6297.1 & 28.0 & R-134a \\
\hline 252.3 & 0.16 & 0.08 & 0.000147 & 0.0208 & 6266.6 & 20.0 & R-134a \\
\hline 248.4 & 0.20 & 0.08 & 0.000161 & 0.0208 & 6262.4 & 19.0 & \\
\hline 238.1 & 0.27 & 0.08 & 0.000183 & 0.0207 & 6249.8 & 18.0 & R-134a \\
\hline 226.5 & 0.35 & 0.08 & 0.000205 & 0.0207 & 6232.1 & 18.0 & R-134a \\
\hline 213.9 & 0.44 & 0.08 & 0.000227 & 0.0207 & 6207.6 & 18.0 & R-134a \\
\hline 199.9 & 0.55 & 0.08 & & & & 19.0 & \\
\hline 186.6 & 0.11 & 0.08 & 0.000078 & 0.0208 & 6309.8 & 29.0 & R-134a \\
\hline 251.8 & 0.16 & 0.08 & 0.000147 & 0.0207 & 6276.8 & 20.0 & R-134a \\
\hline 246.7 & 0.20 & 0.08 & 0.000161 & 0.0207 & 6272.1 & 19.0 & R-134a \\
\hline 235.6 & 0.28 & 0.08 & 0.000184 & 0.0207 & 6258.7 & 18.0 & R-134a \\
\hline 223.4 & 0.35 & 0.08 & 0.000205 & 0.0207 & 6239.8 & 18.0 & R-134a \\
\hline 210.1 & 0.45 & 0.08 & 0.000227 & 0.0205 & 6213.9 & 18.0 & R-134a \\
\hline 195.9 & 0.56 & 0.08 & 0.000251 & 0.0206 & 6177.4 & 19.0 & R-134a \\
\hline 241.9 & 0.20 & 0.08 & 0.000169 & 0.0207 & 3901.0 & 26.0 & R-134a \\
\hline 238.2 & 0.24 & 0.08 & 0.000184 & 0.0207 & 3899.3 & 24.0 & R-134a \\
\hline 230.7 & 0.33 & 0.08 & 0.000210 & 0.0207 & 3895.1 & 23.0 & R-134a \\
\hline 222.9 & 0.42 & 0.08 & 0.000235 & 0.0207 & 3889.9 & 22.0 & R-134a \\
\hline 214.6 & 0.52 & 0.08 & 0.000260 & 0.0207 & 3882.9 & 23.0 & R-134a \\
\hline 205.8 & 0.65 & 0.08 & 0.000288 & 0.0207 & 3873.5 & 24.0 & R-134a \\
\hline 204.1 & 0.79 & 0.08 & 0.000191 & 0.0208 & 2659.5 & 29.0 & $\mathrm{R}-134 \mathrm{a}$ \\
\hline 200.1 & 0.87 & 0.08 & 0.000213 & 0.0208 & 2658.1 & 28.0 & R-134a \\
\hline 195.7 & 0.96 & 0.08 & 0.000236 & 0.0208 & 2656.3 & 29.0 & $\mathrm{R}-134 \mathrm{a}$ \\
\hline 191.2 & 0.99 & 0.08 & 0.000262 & 0.0208 & 2654.0 & 30.0 & R-134a \\
\hline
\end{tabular}

